\title{
Conformational Properties of Glycosaminoglycan Disaccharides: A Molecular Dynamics Study
}

Valery Lutsyk, Wojciech Plazinski

Jerzy Haber Institute of Catalysis and Surface Chemistry

Polish Academy of Sciences

Niezapominajek 8, 30-239 Krakow, Poland

*Corresponding author; e-mail: wojtek plazinski@o2.pl

[Supporting Information] 
Tab. S1. The chemical composition of GAG disaccharides considered in this work.

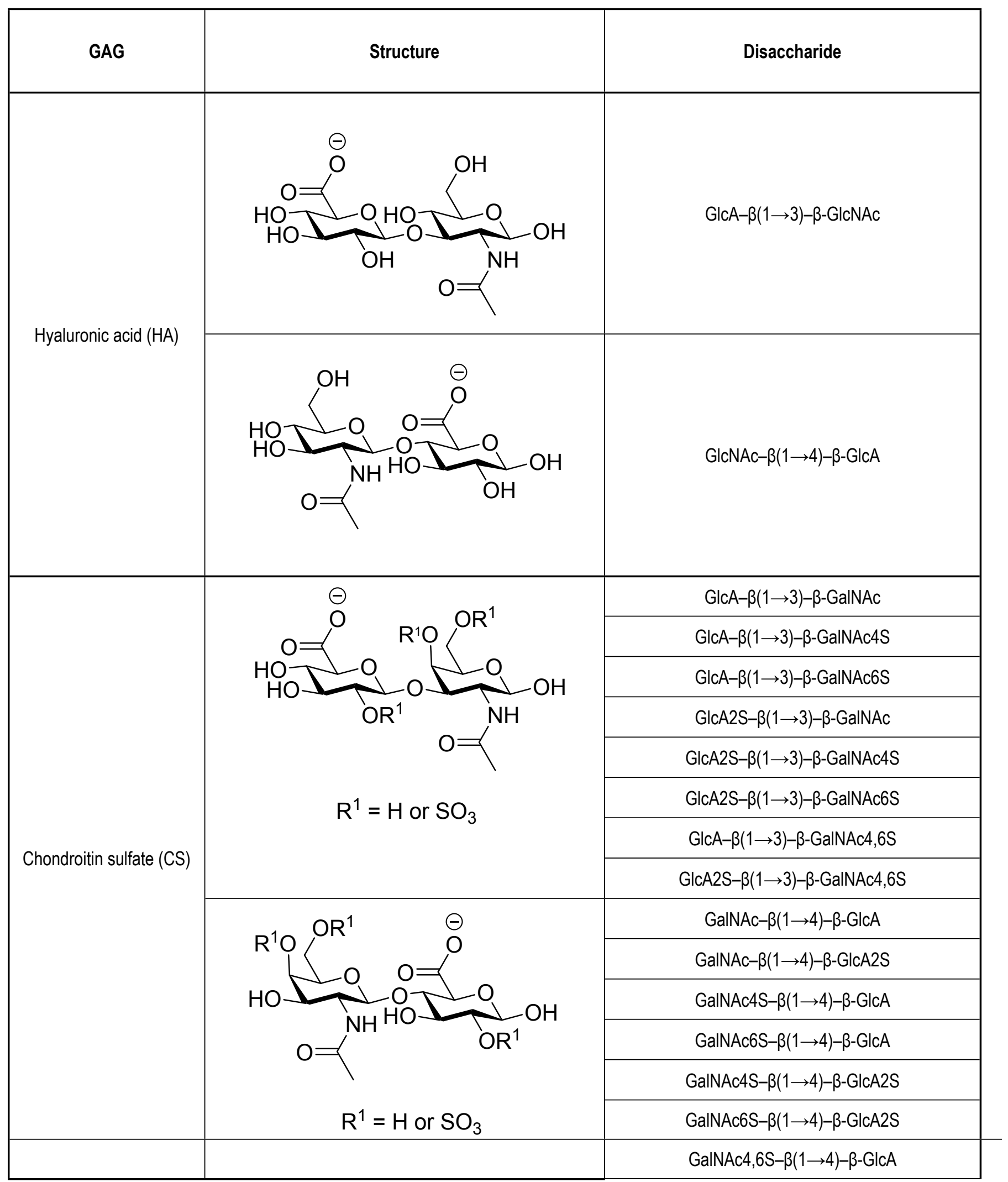




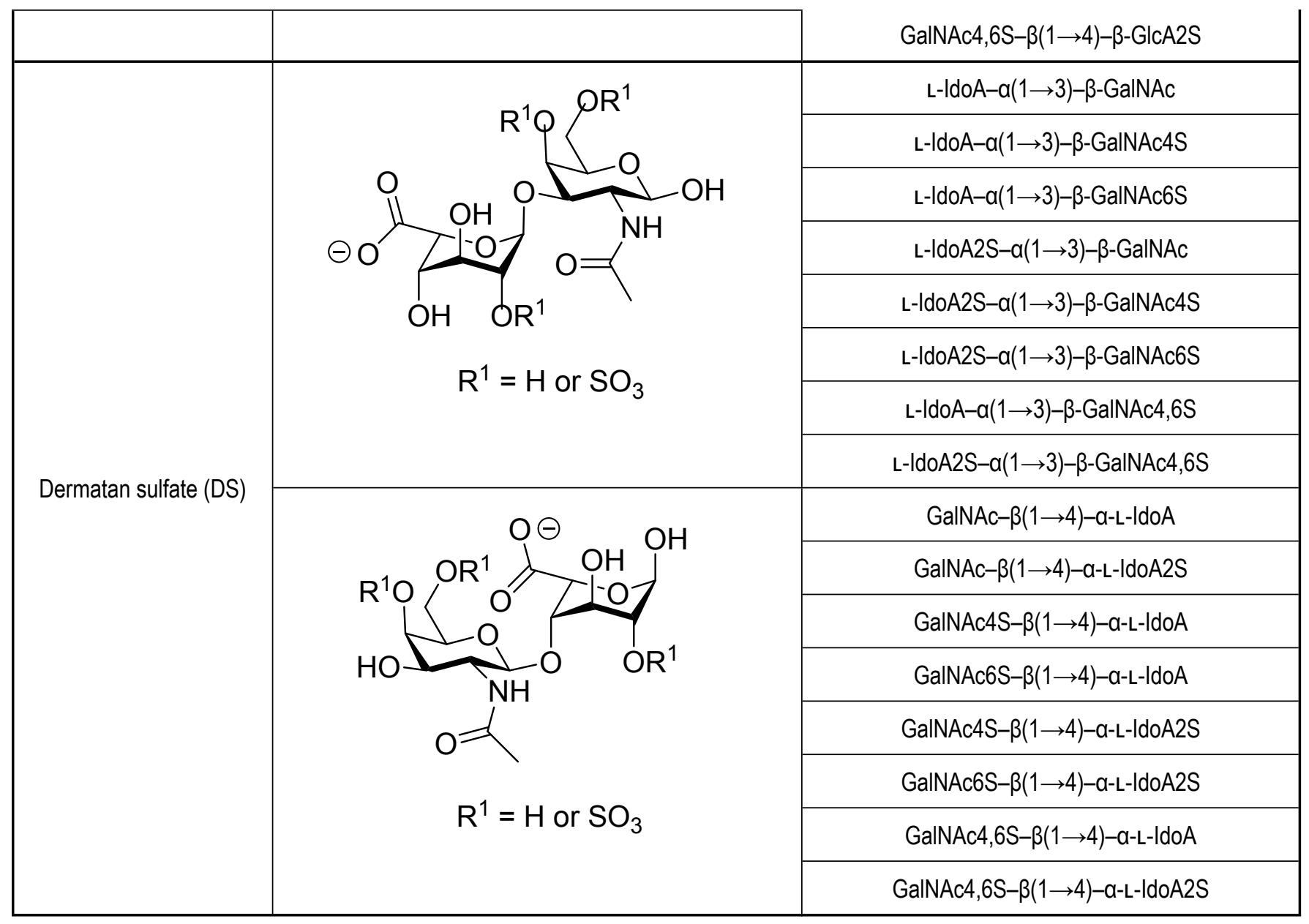




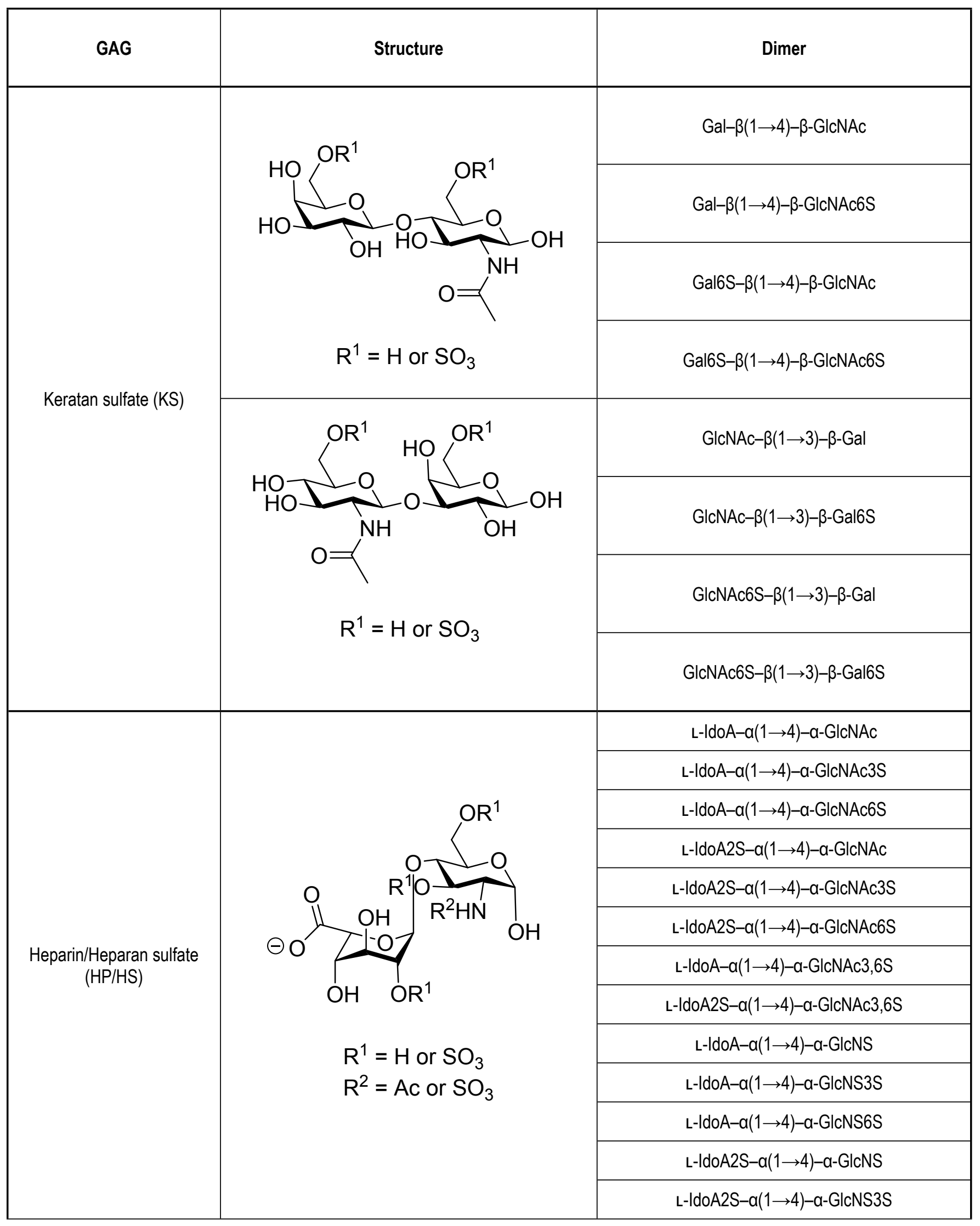




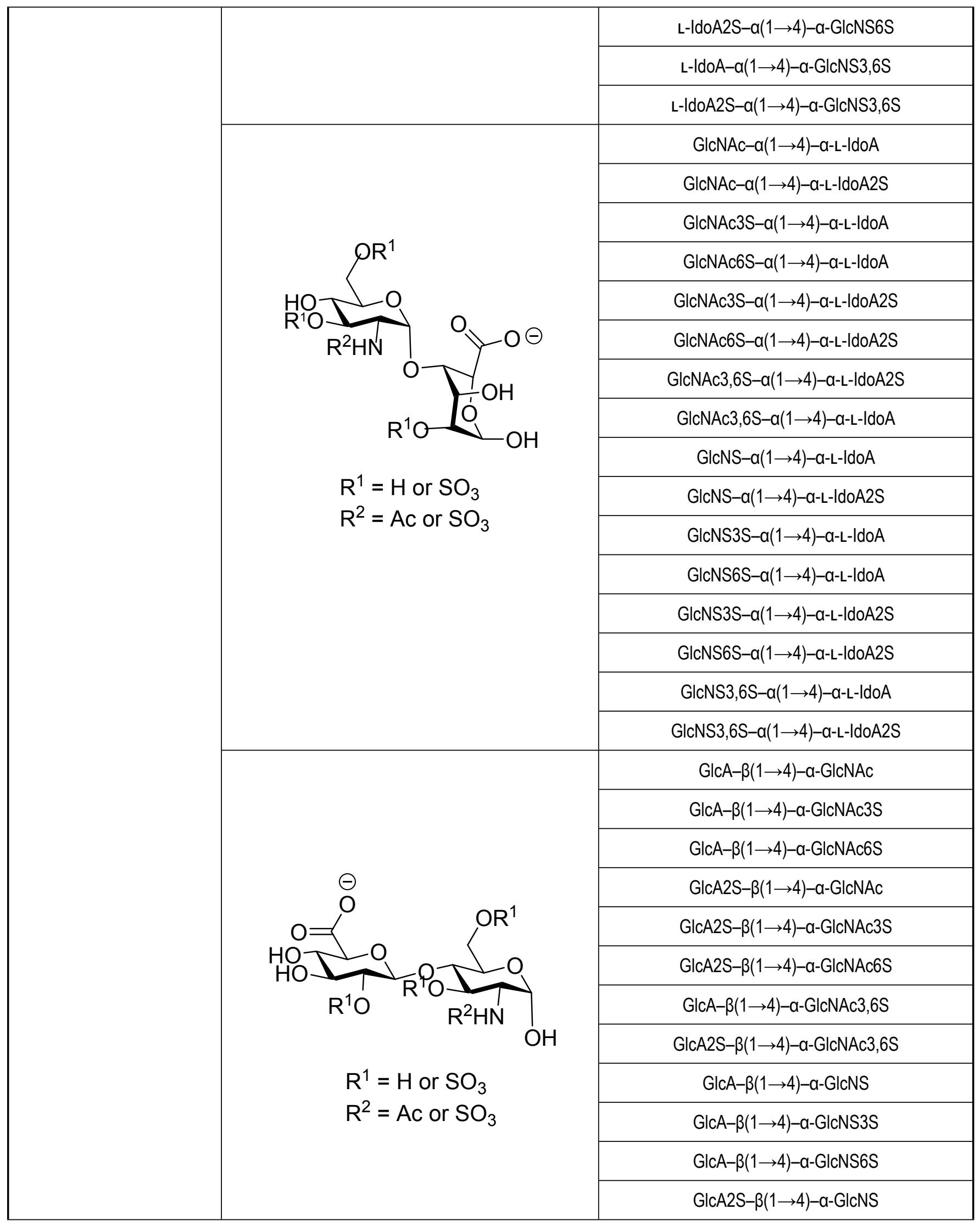




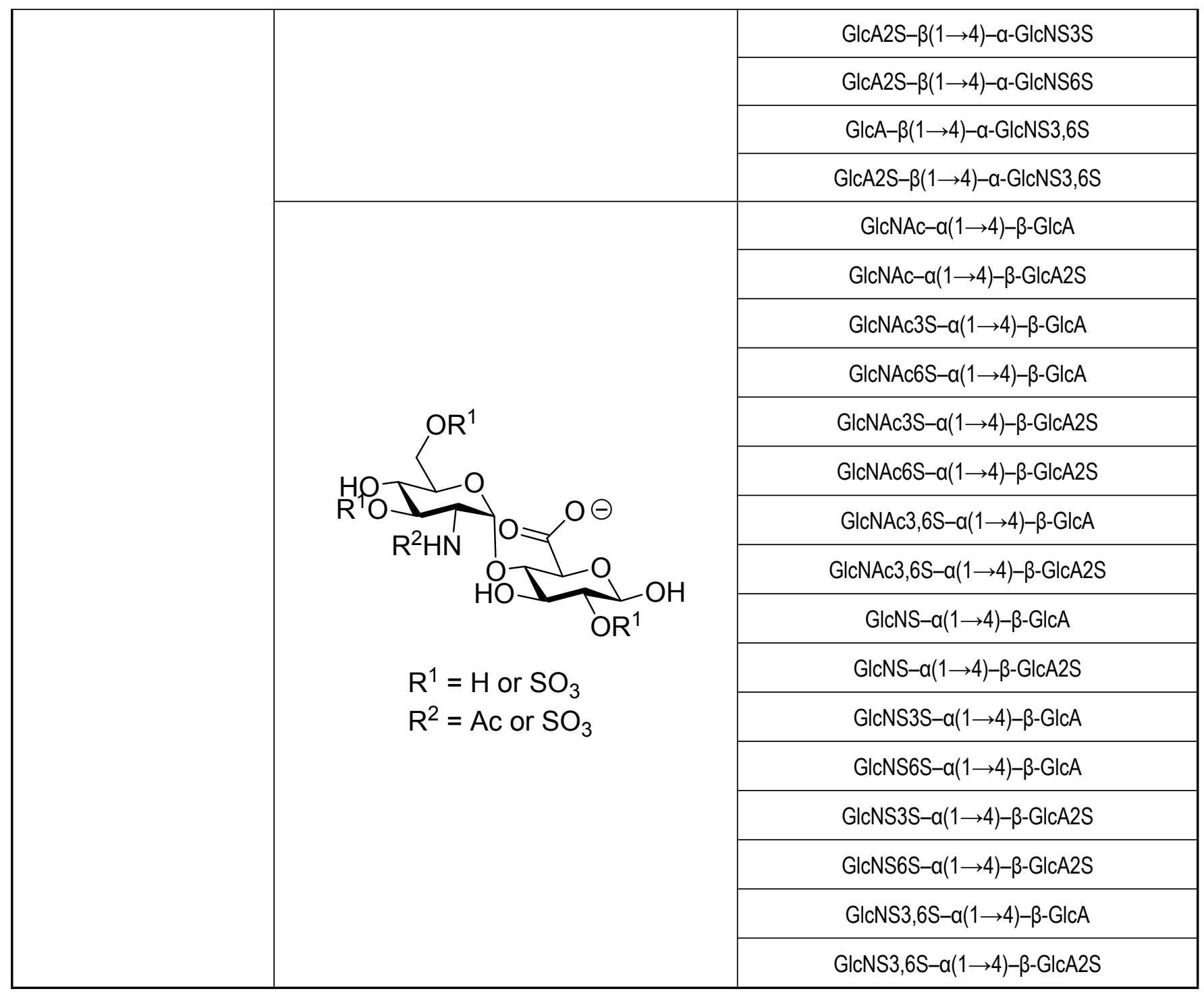




\section{Free energy maps (Fig. S1)}

\section{Hyaluronic acid (HA)}

a. GlcA- $\beta(1 \rightarrow 3)-\beta-G l c N A c$

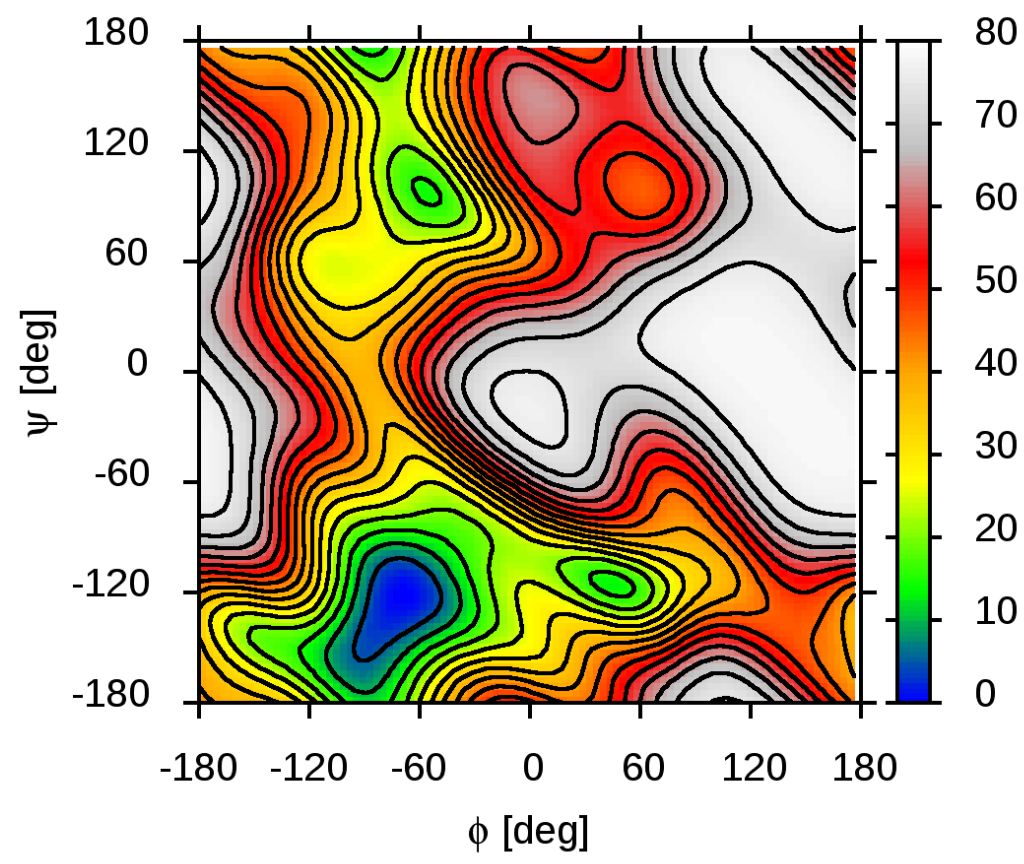

b. GlcNAc- $\beta(1 \rightarrow 4)-\beta-G l c A$

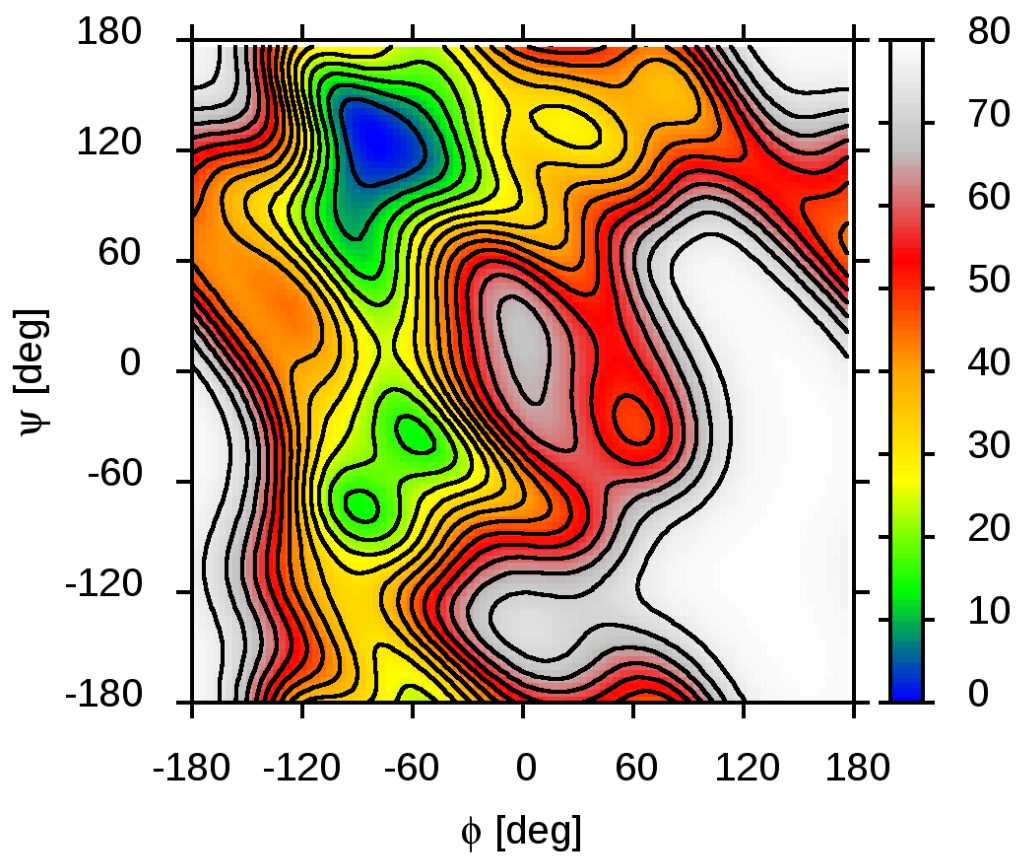

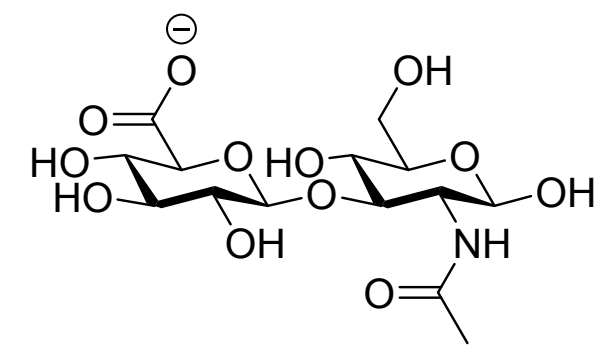




\section{Chondroitin sulfate (CS)}

a. GlcA2R ${ }^{1}-\beta(1 \rightarrow 3)-\beta-G a l N A c 4,6 R^{1}$

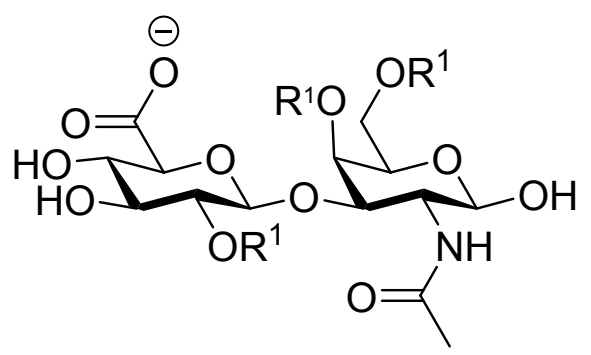

$$
\mathrm{R}^{1}=\mathrm{H} \text { or } \mathrm{SO}_{3}
$$

1. $\operatorname{Glc} A-\beta(1 \rightarrow 3)-\beta-$ GalNAc

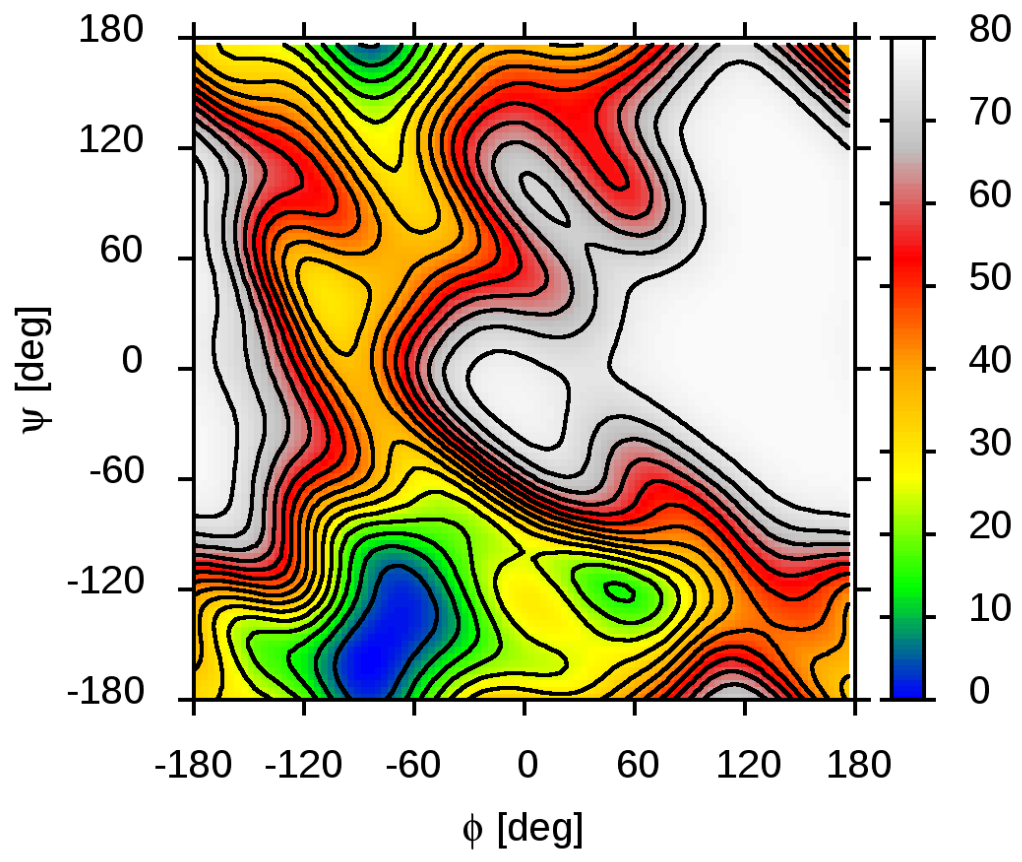


2. GlcA- $\beta(1 \rightarrow 3)-\beta-$ GalNAc4S

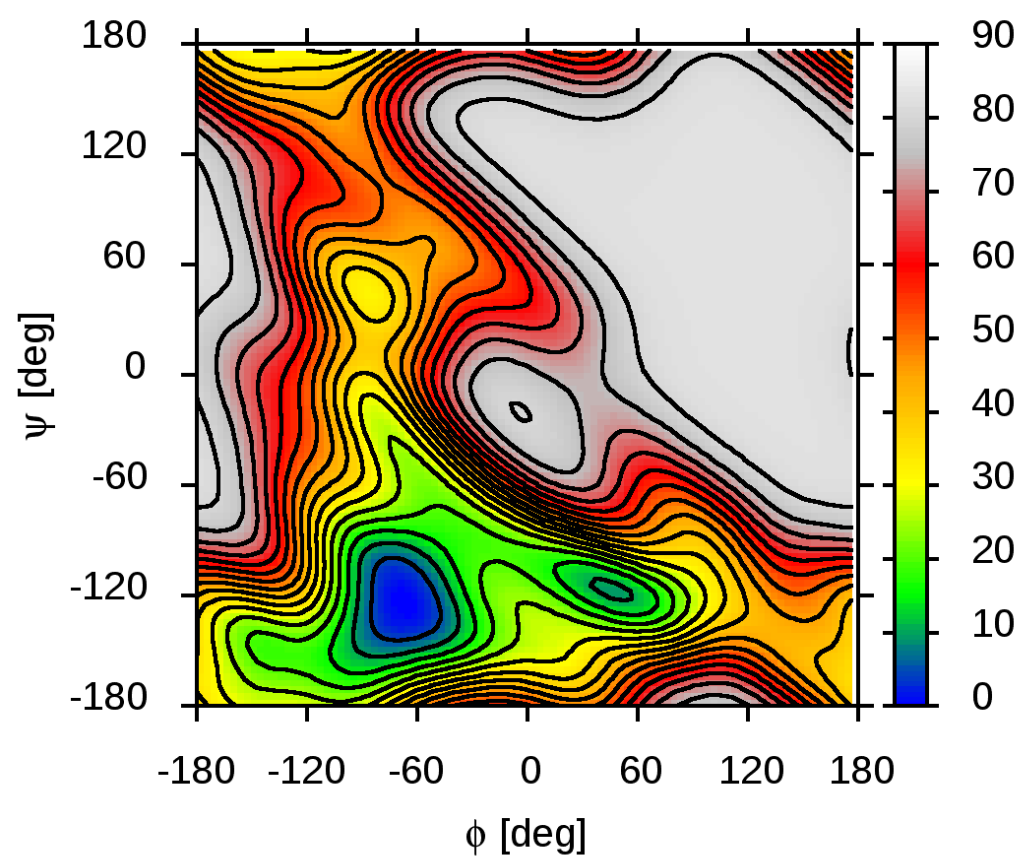

3. $\operatorname{GlcA}-\beta(1 \rightarrow 3)-\beta-$ GalNAc6S

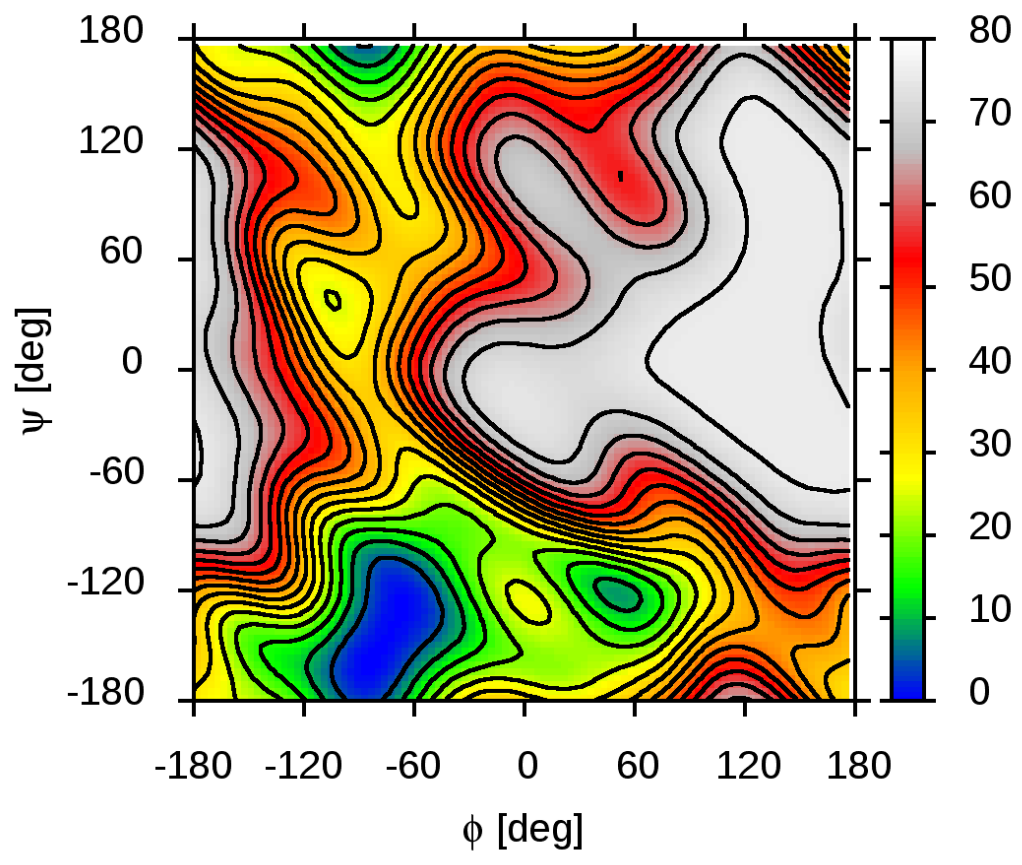


4. GlcA2S- $\beta(1 \rightarrow 3)-\beta-$ GalNAc

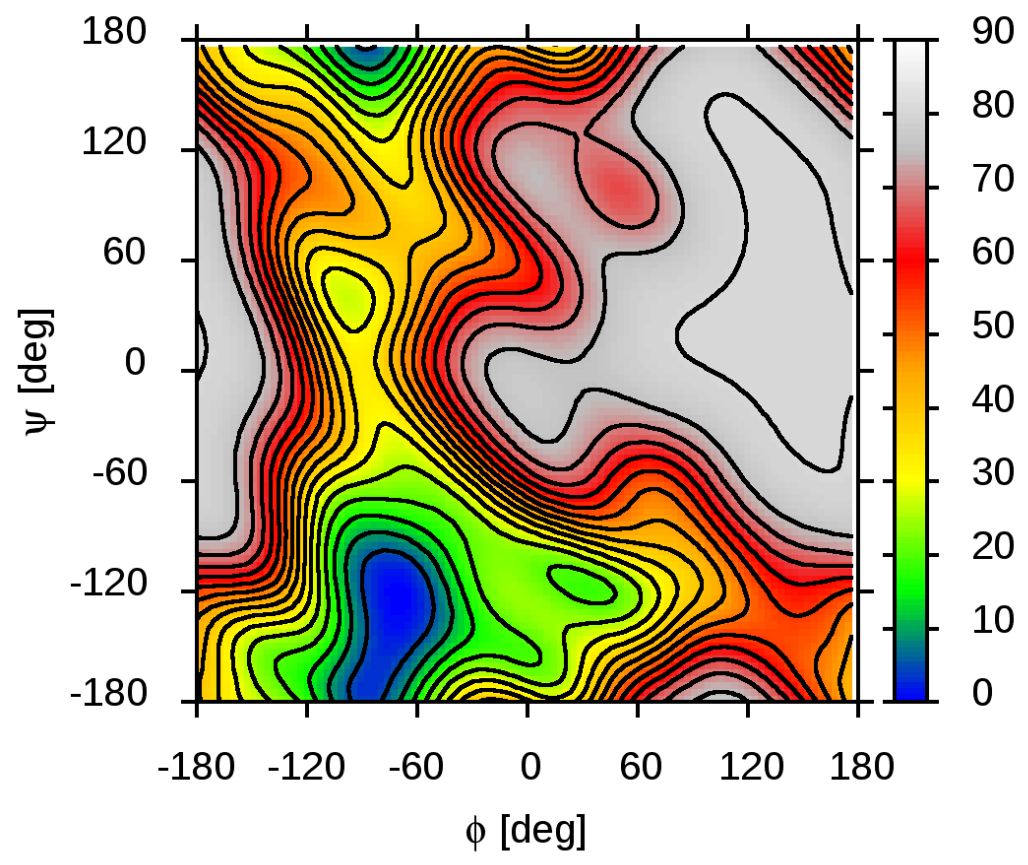

5. GlcA2S- $\beta(1 \rightarrow 3)-\beta-$ GalNAc4S

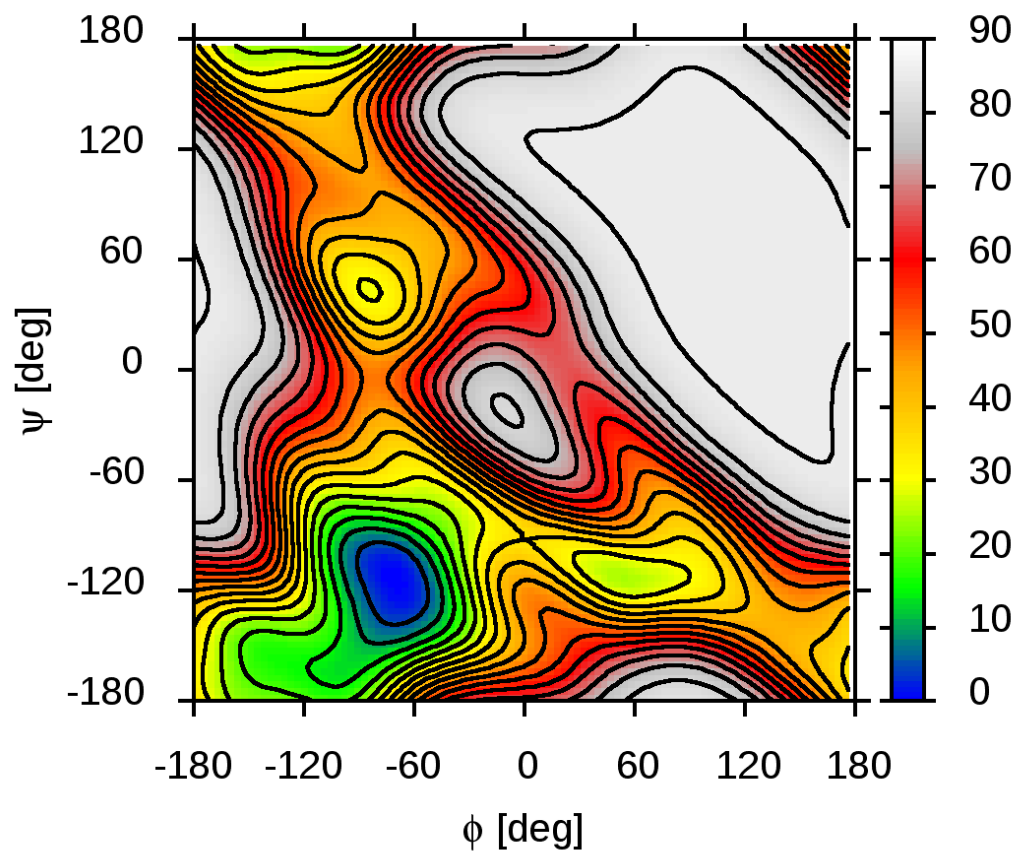


6. GlcA2S- $\beta(1 \rightarrow 3)-\beta-$ GalNAc6S

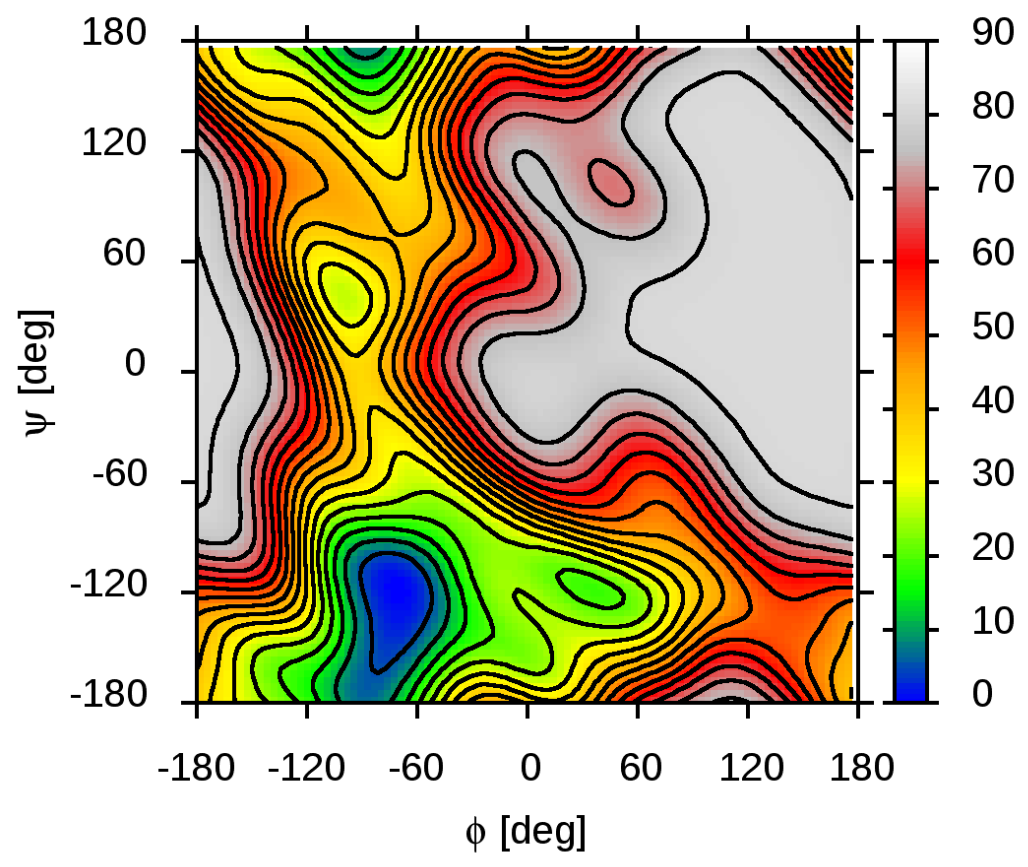

7. GlcA- $\beta(1 \rightarrow 3)-\beta-$ GalNAc4,6S

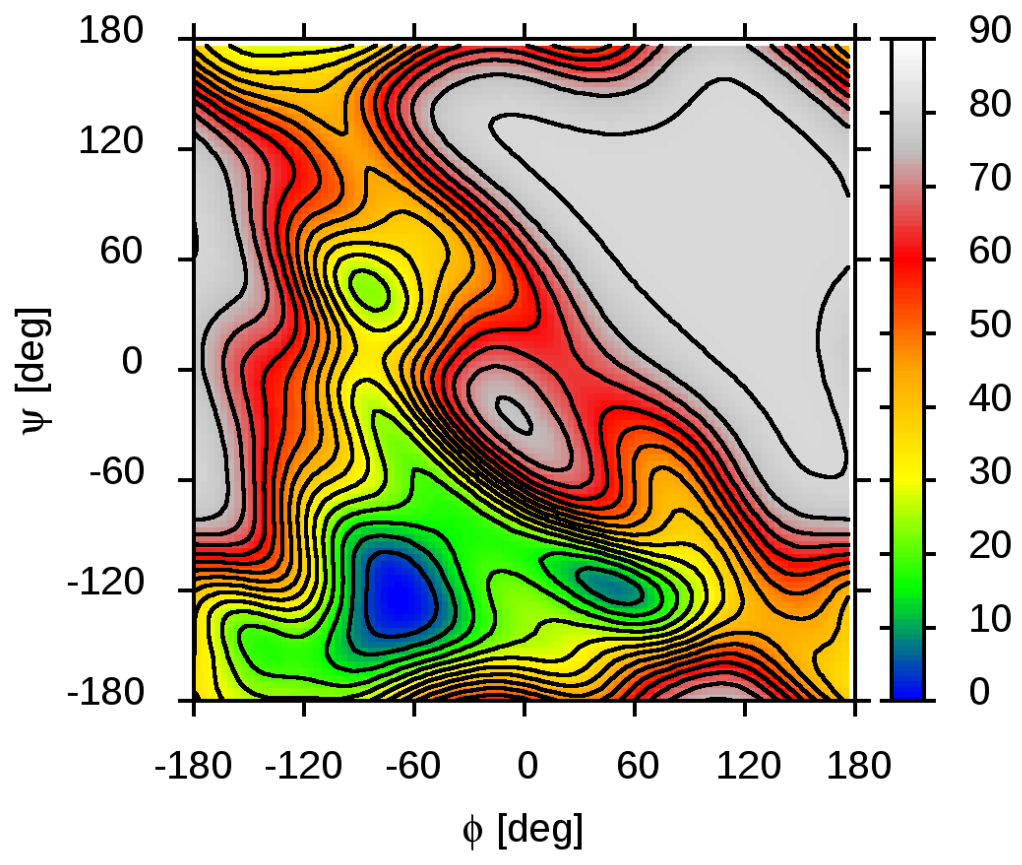


8. GlcA2S- $\beta(1 \rightarrow 3)-\beta-$ GalNAc4,6S

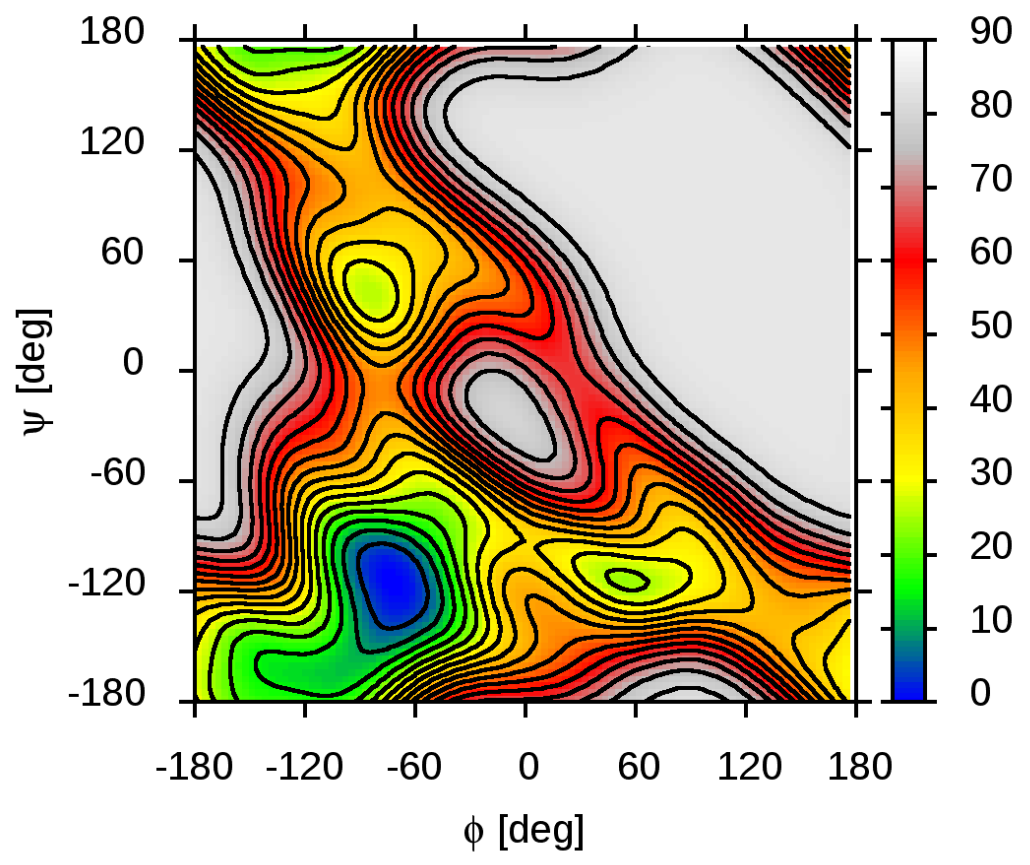

b. GalNAc4,6R ${ }^{1}-\beta(1 \rightarrow 4)-\beta-G \mid c A 2 R^{1}$

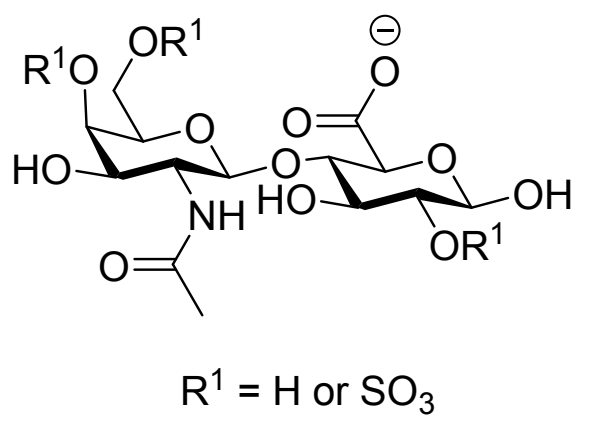


1. GalNAc- $\beta(1 \rightarrow 4)-\beta-G \mid c A$

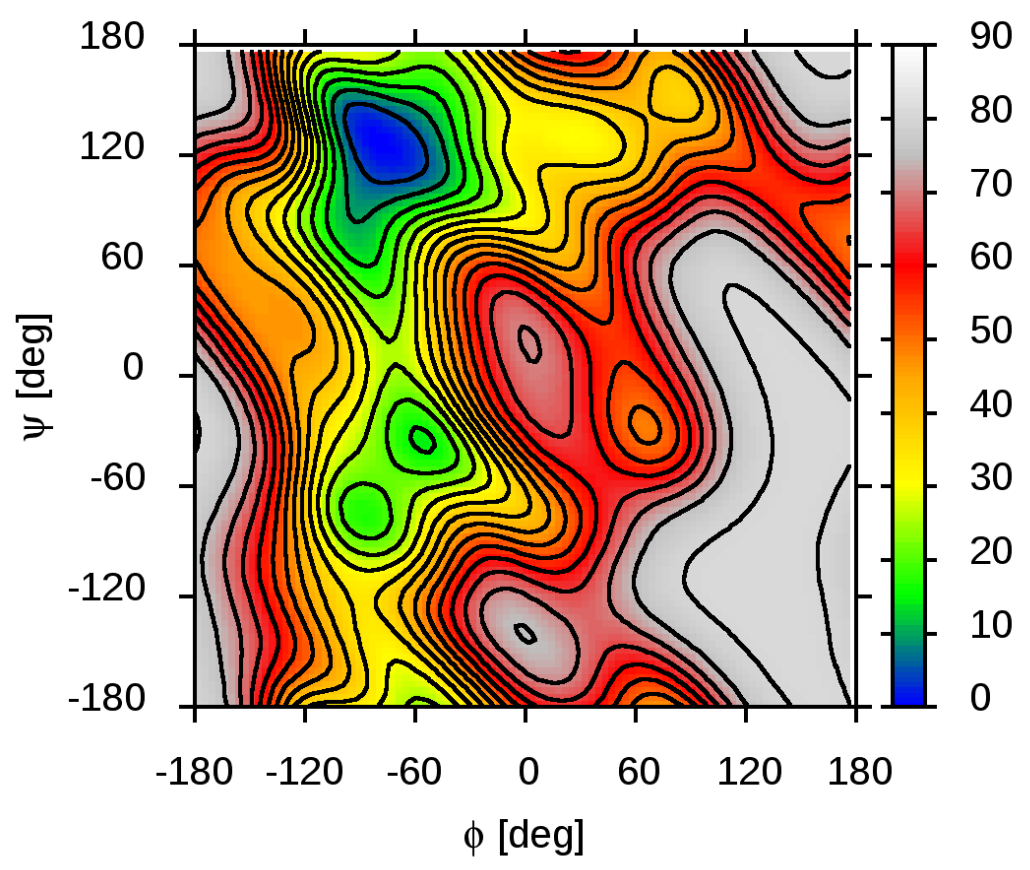

2. GalNAc- $\beta(1 \rightarrow 4)-\beta-$ GlcA2S

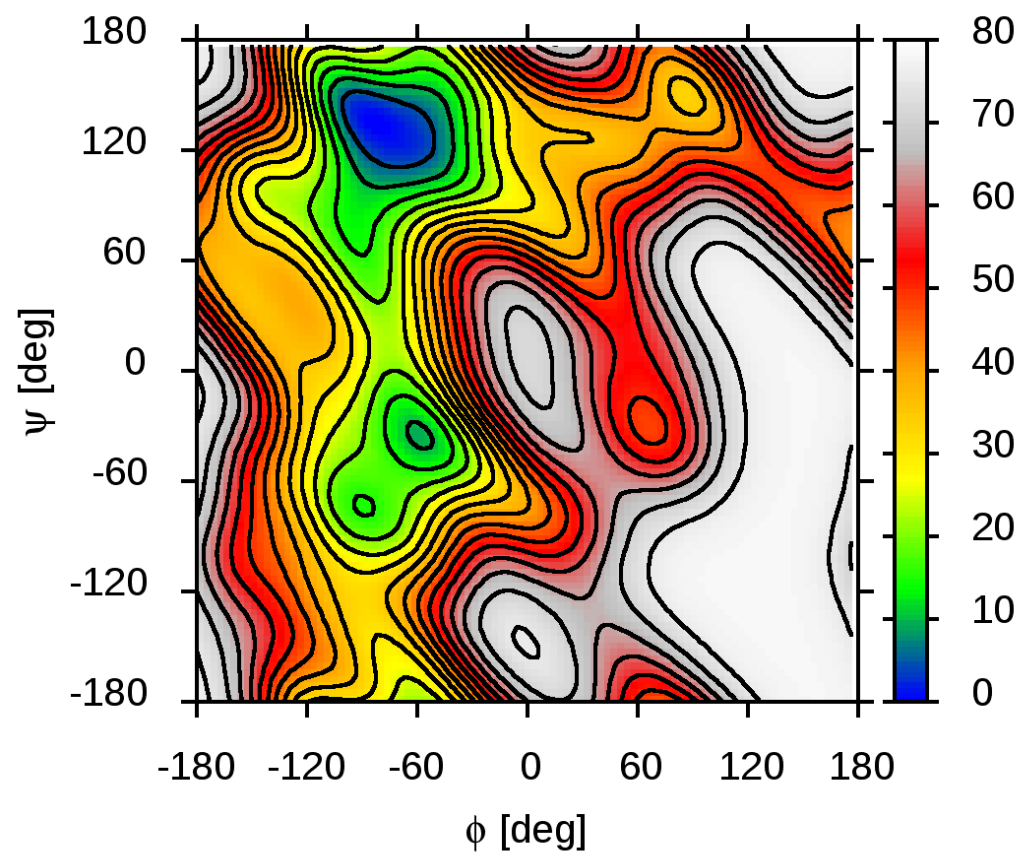


3. GalNAc4S- $\beta(1 \rightarrow 4)-\beta-G l c A$

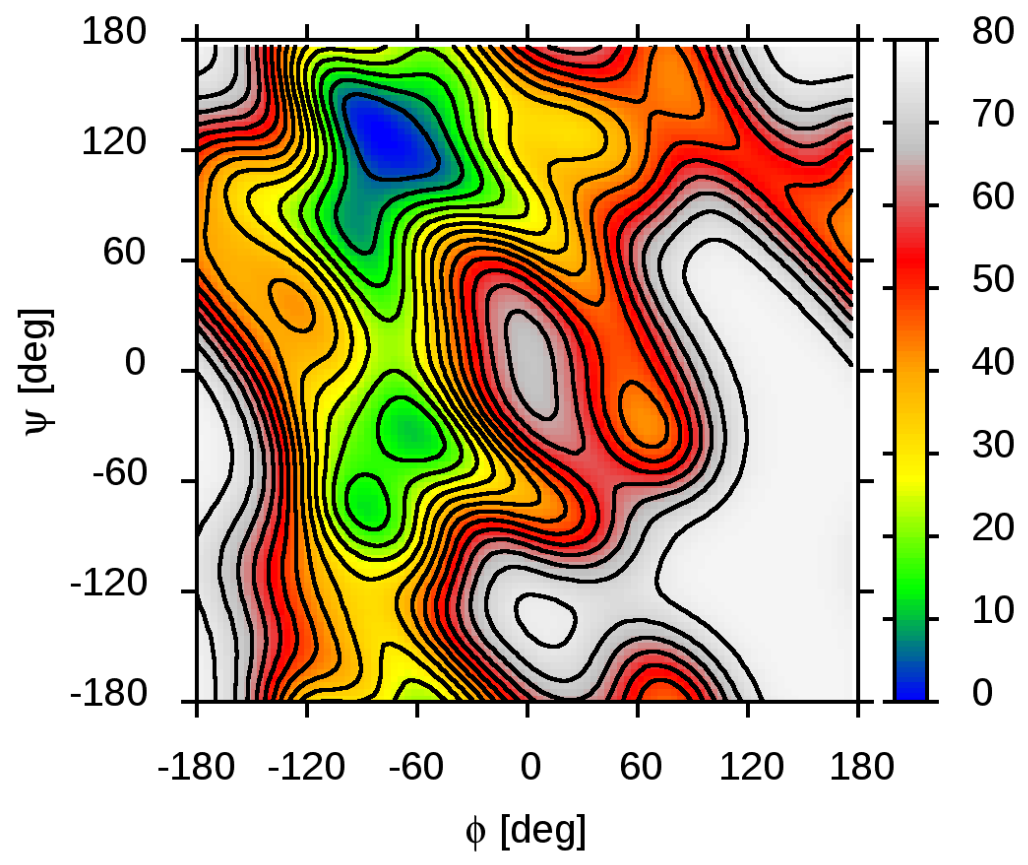

4. GalNAc6S- $\beta(1 \rightarrow 4)-\beta-$ GlcA

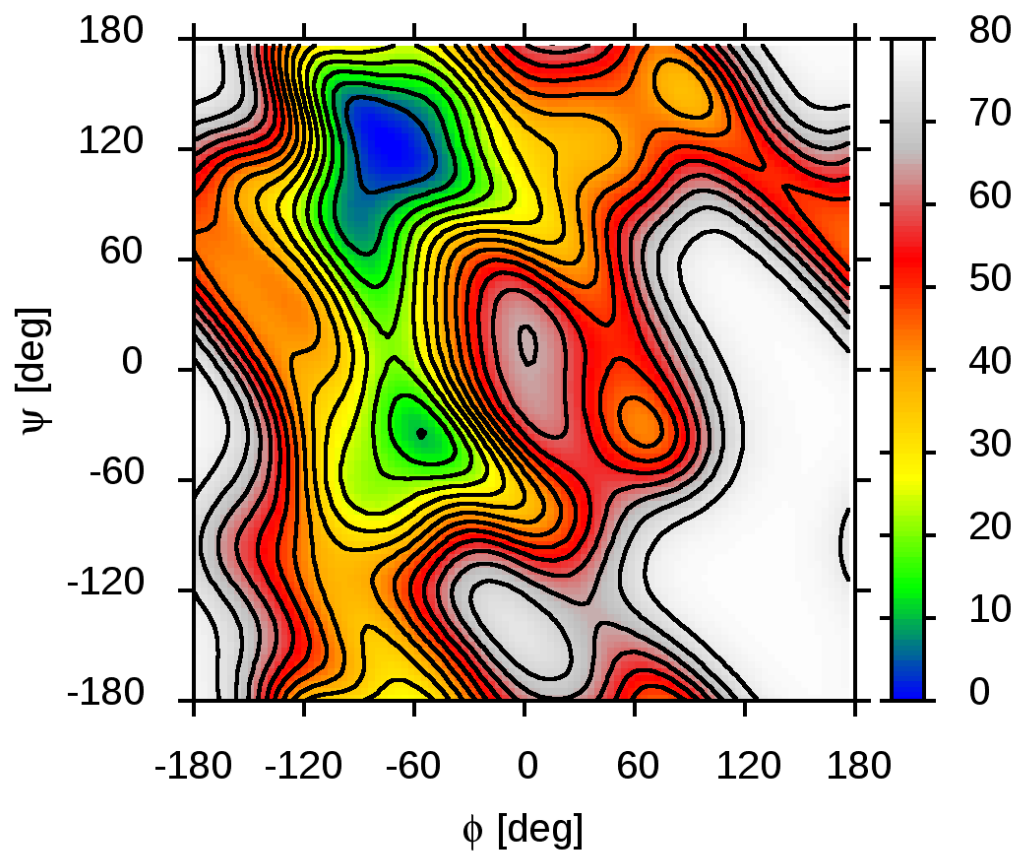


5. GalNAc4S- $\beta(1 \rightarrow 4)-\beta-$ GlcA2S

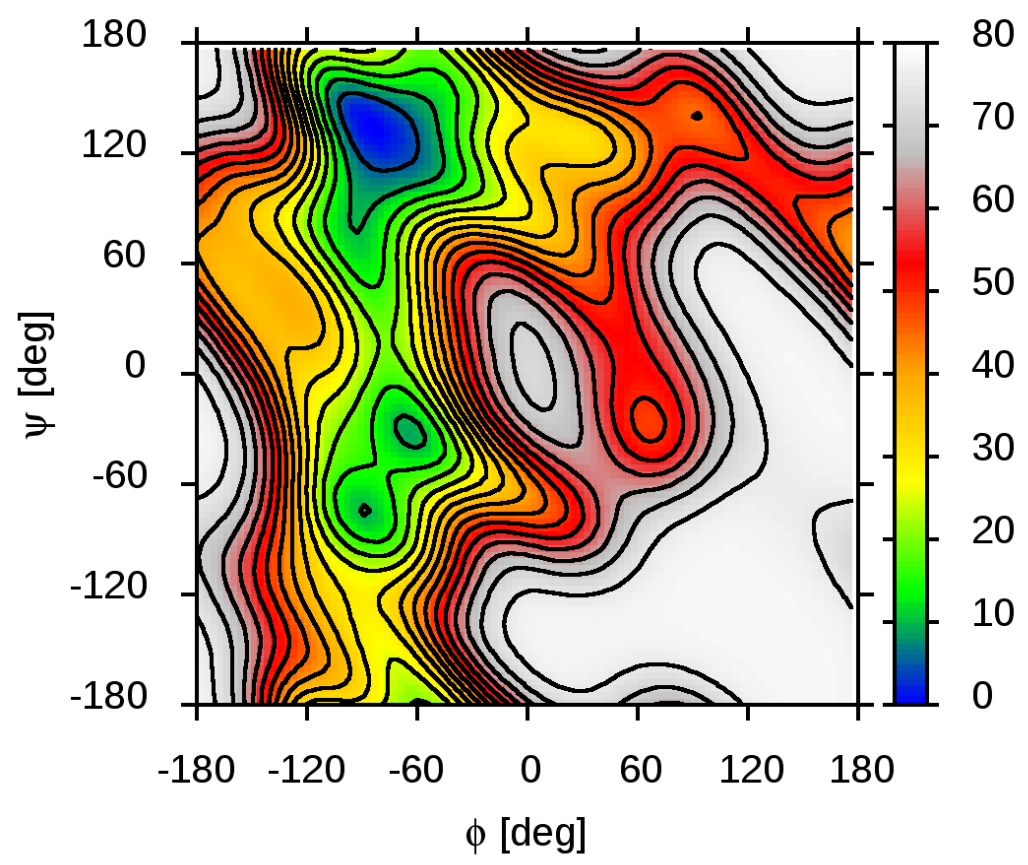

6. GalNAc6S- $\beta(1 \rightarrow 4)-\beta-G l c A 2 S$

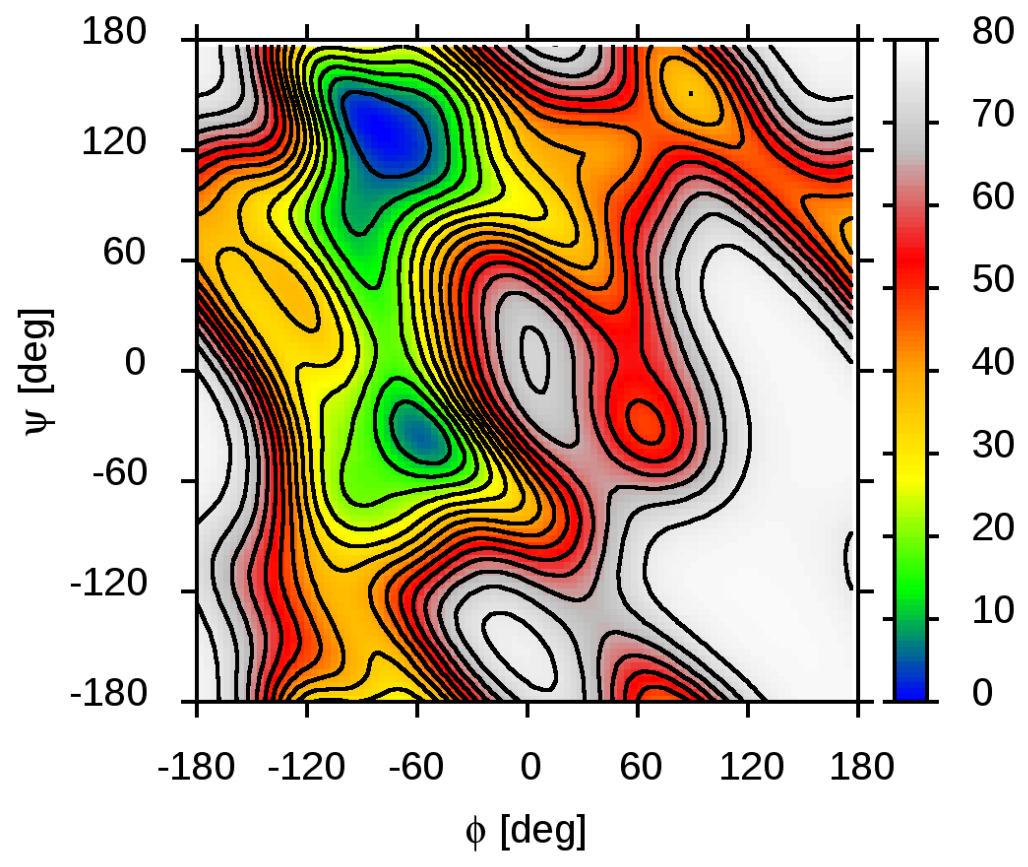


7. GalNAc4,6S- $\beta(1 \rightarrow 4)-\beta-G \mid c A$

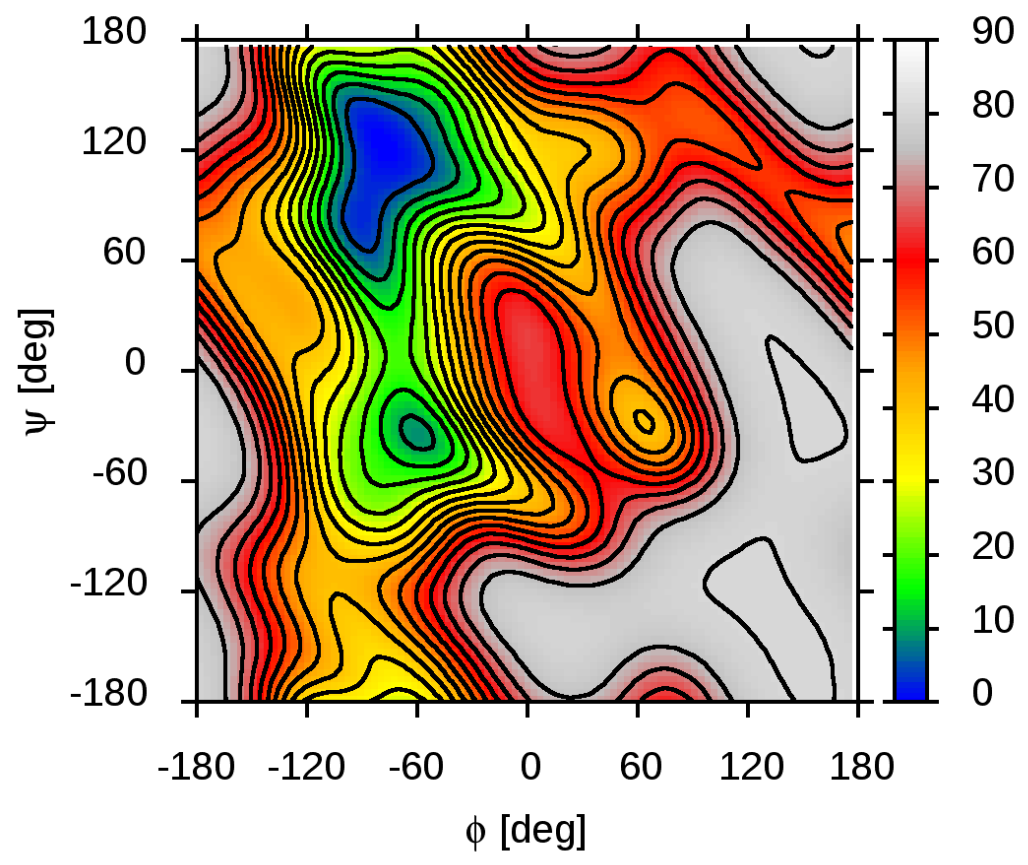

8. GalNAc4,6S- $\beta(1 \rightarrow 4)-\beta-$-GlcA2S

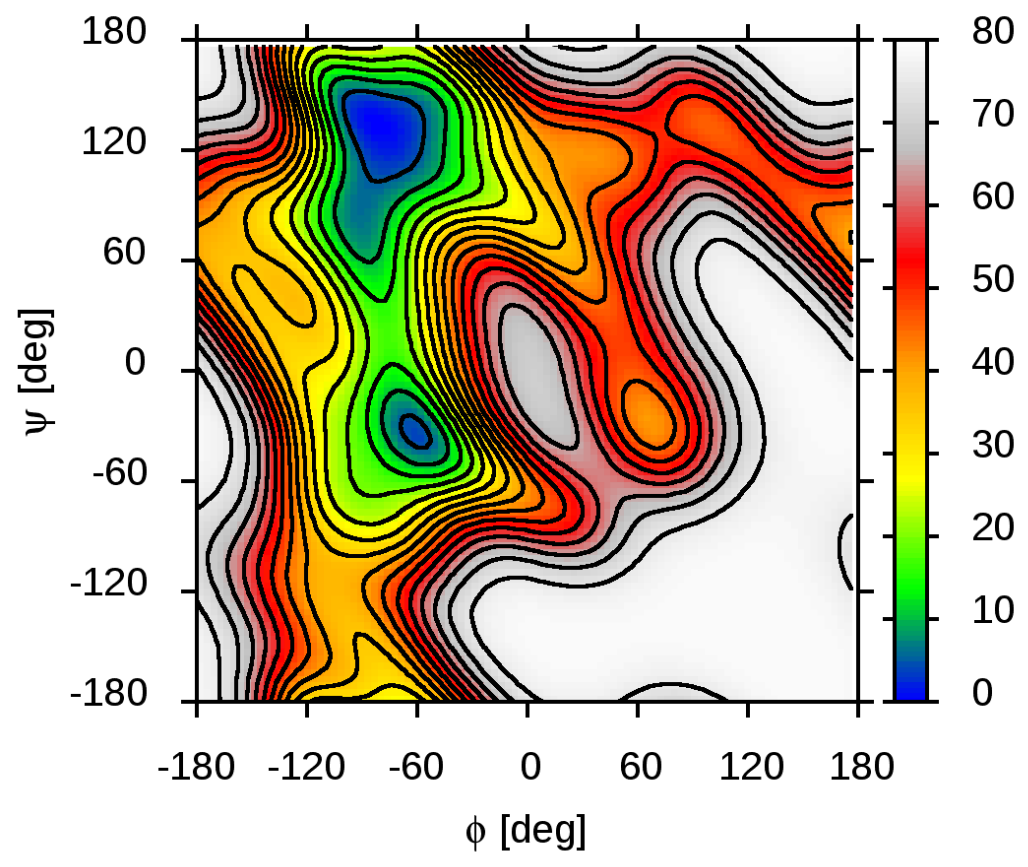




\section{Dermatan sulfate (DS)}

a. L-IdoA2R ${ }^{1}-\alpha(1 \rightarrow 3)-\beta$-GalNAc4,6R ${ }^{1}$

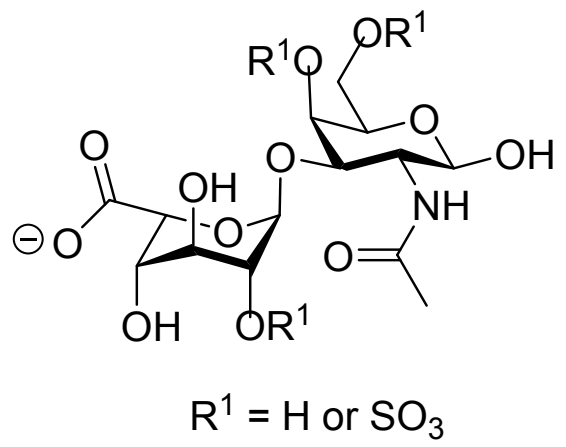

1. L-IdoA- $\alpha(1 \rightarrow 3)-\beta-G a I N A c$

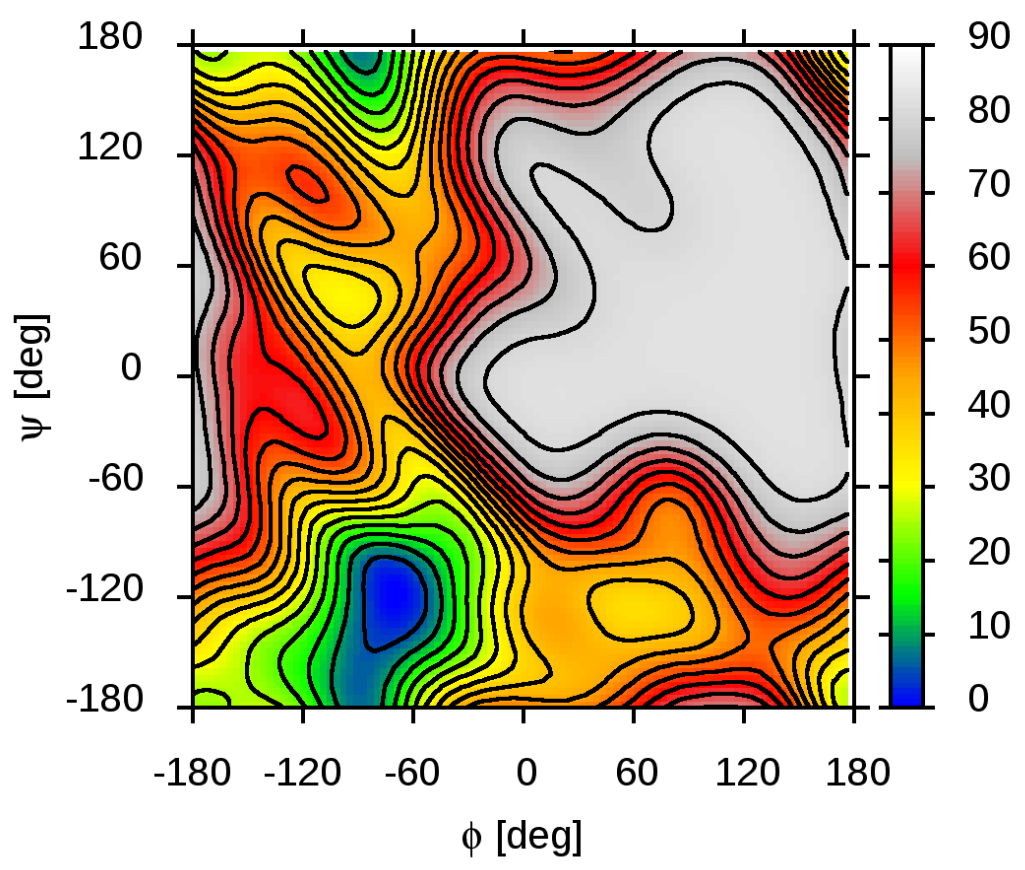


2. L-IdoA- $\alpha(1 \rightarrow 3)-\beta-$ GalNAc4S

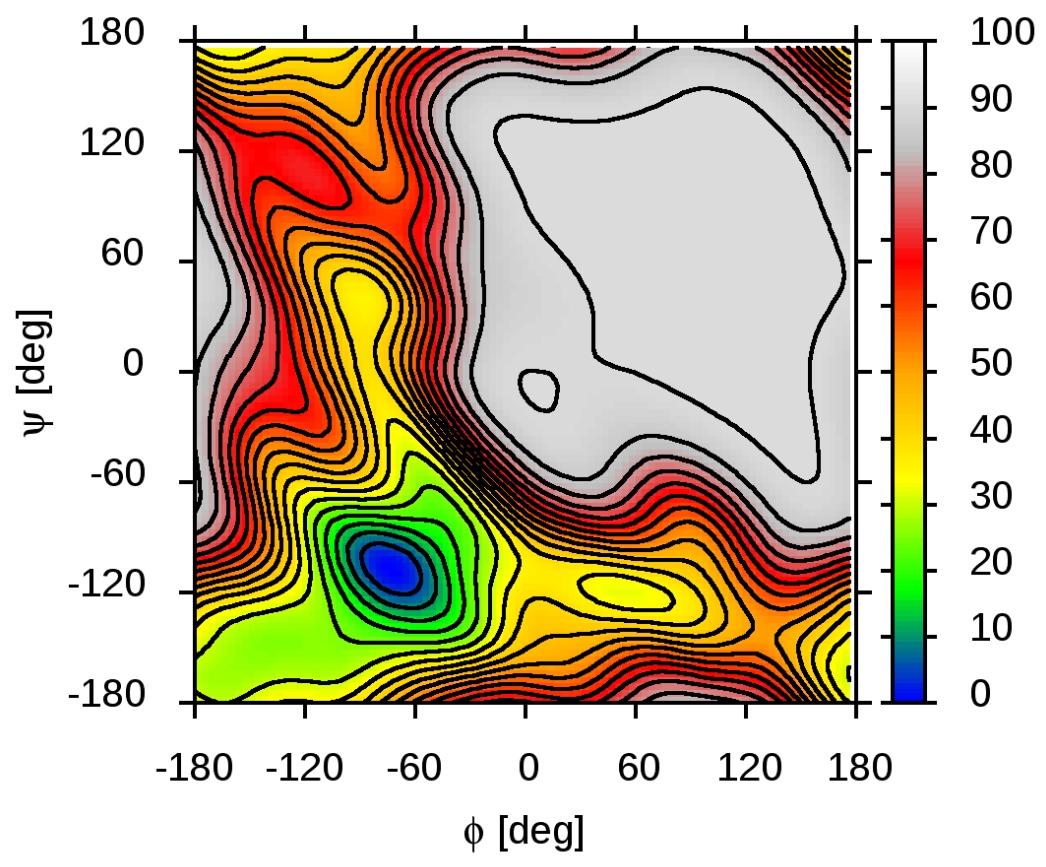

3. L-IdoA- $\alpha(1 \rightarrow 3)-\beta-$-GalNAc6S

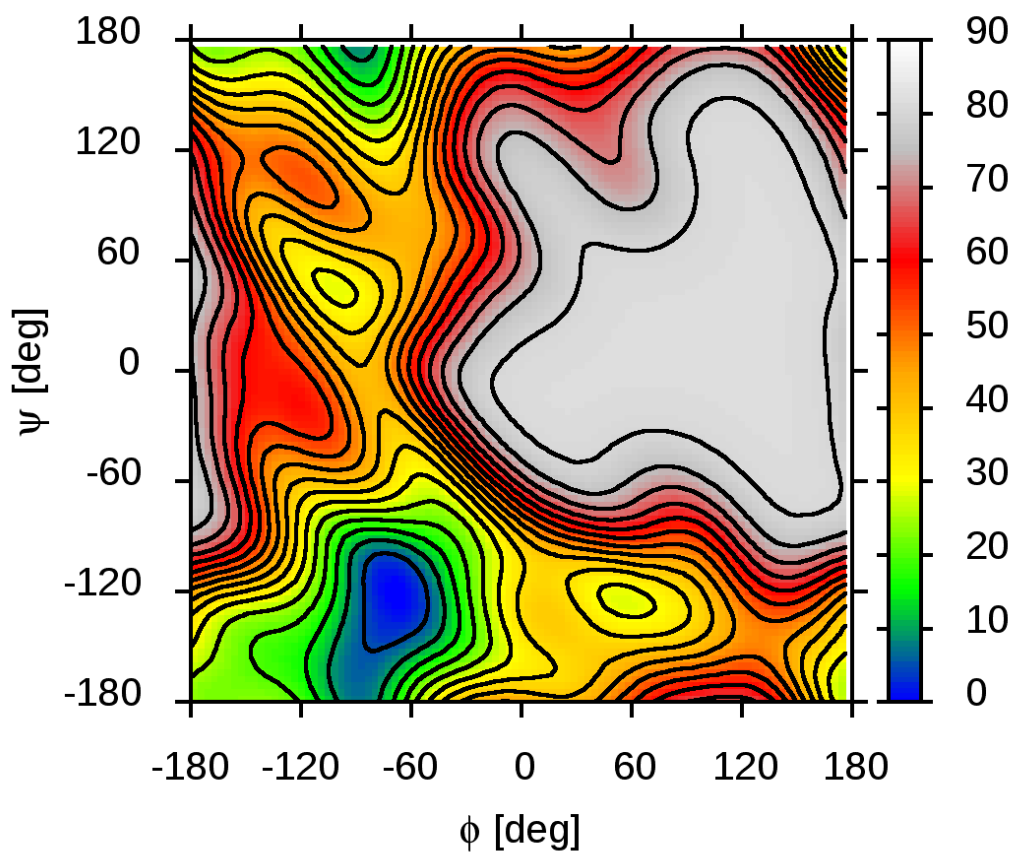


4. L-IdoA2S- $\alpha(1 \rightarrow 3)-\beta$-GalNAc

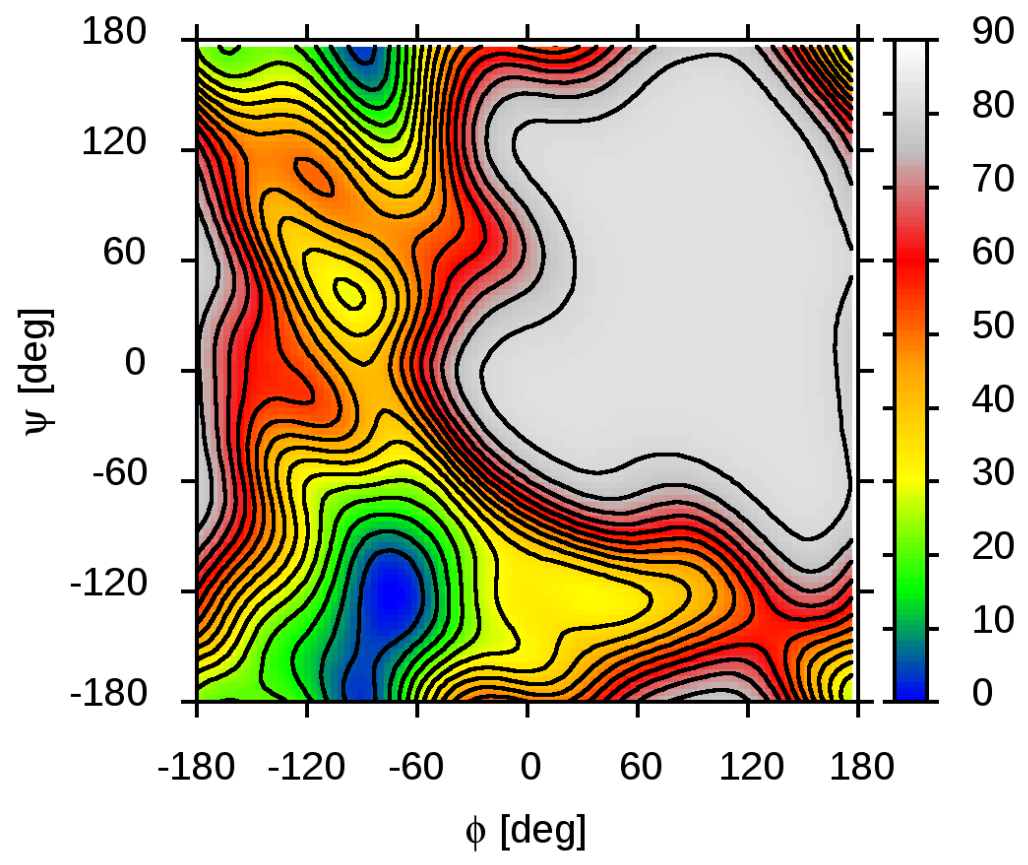

5. L-IdoA2S- $\alpha(1 \rightarrow 3)-\beta-G a I N A c 4 S$

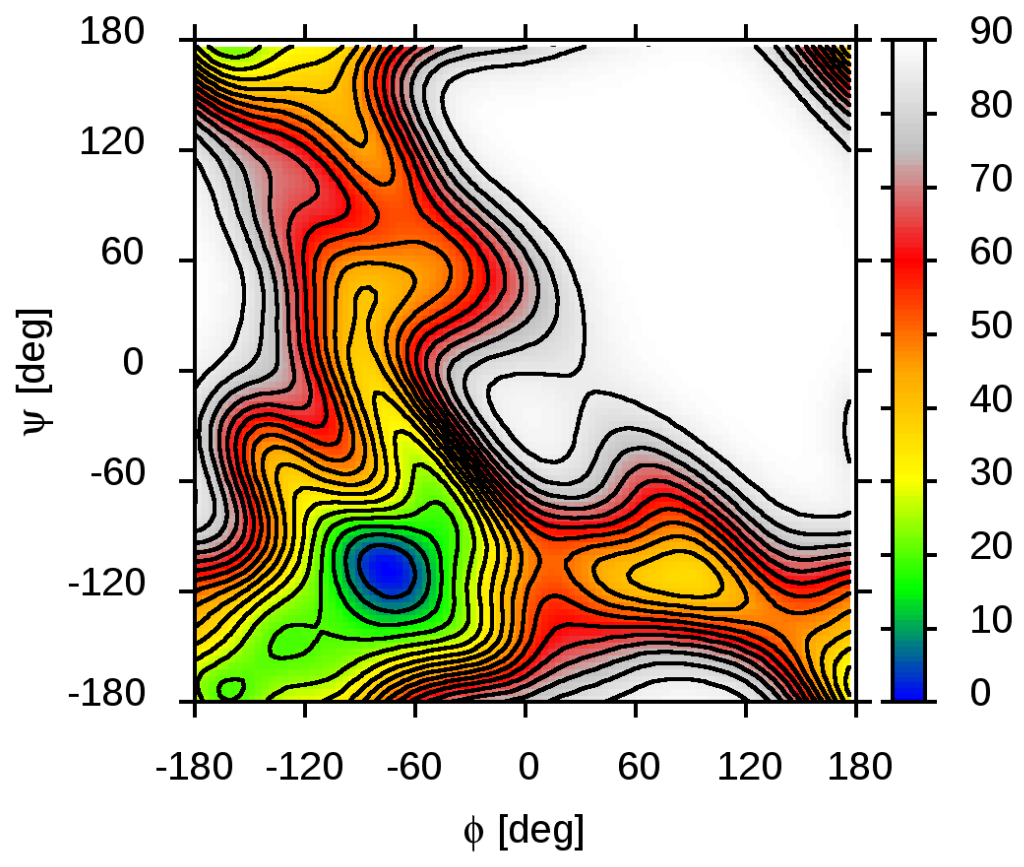


6. L-IdoA2S- $\alpha(1 \rightarrow 3)-\beta-$ GalNAc6S

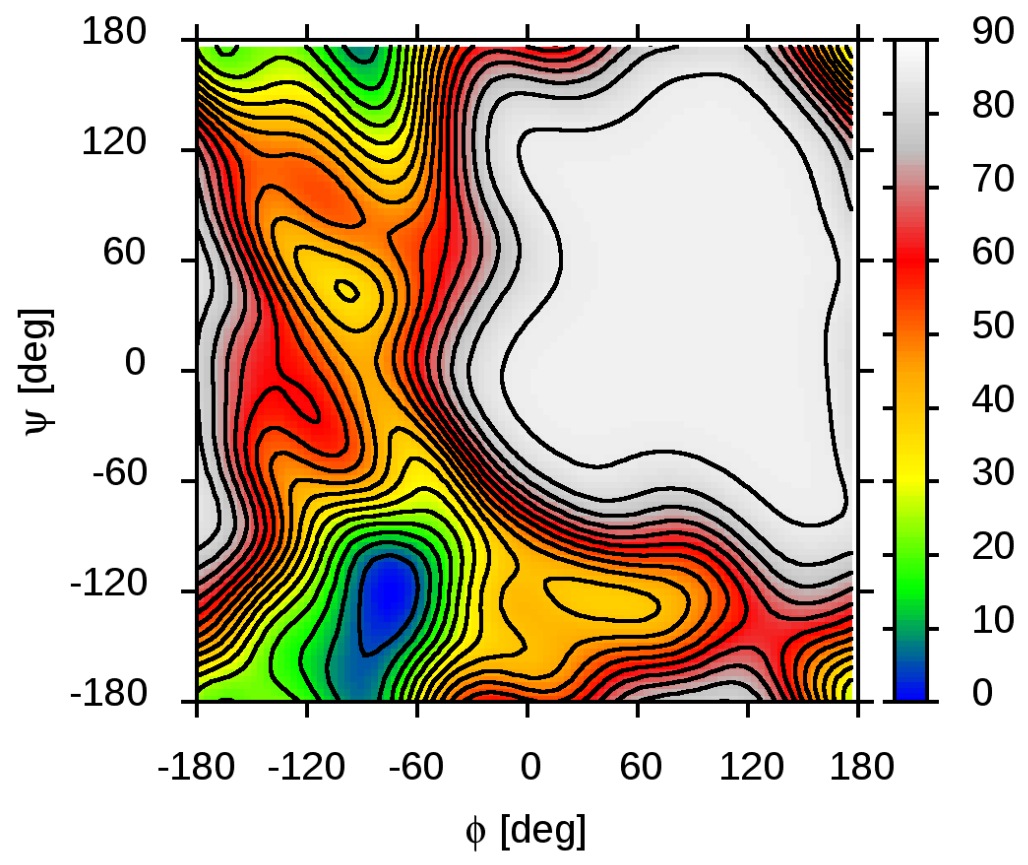

7. L-IdoA- $\alpha(1 \rightarrow 3)-\beta$-GalNAc4,6S

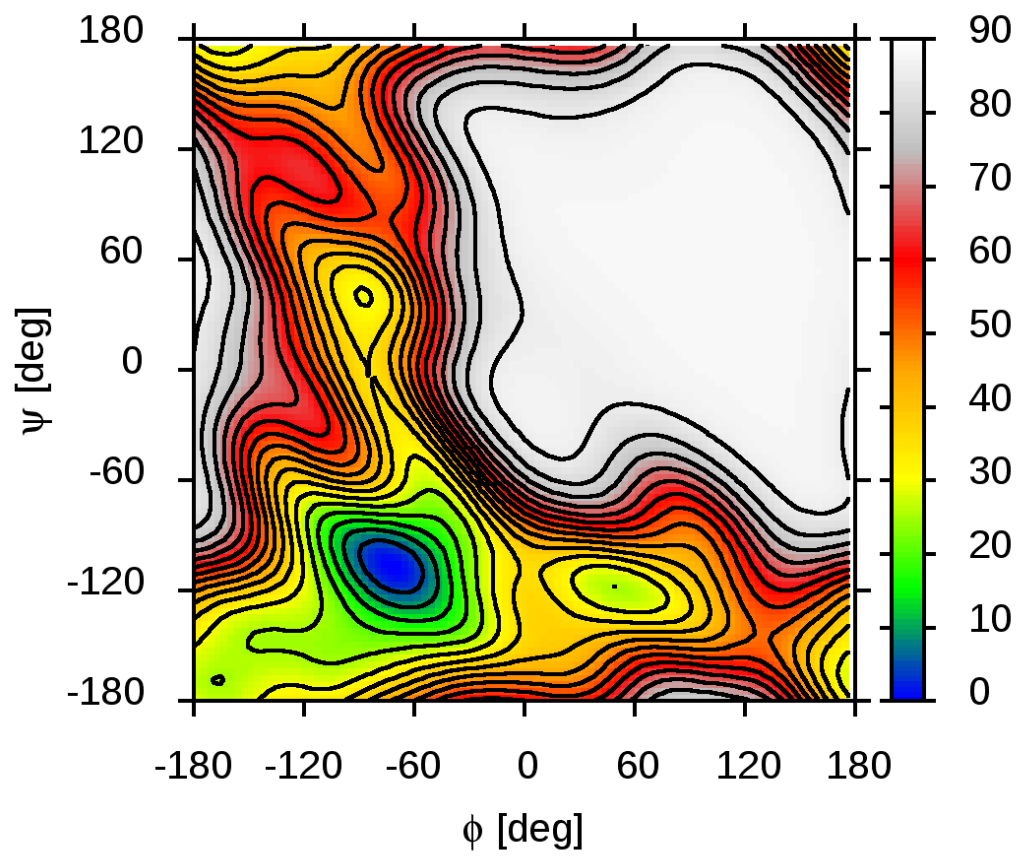


8. L-IdoA2S- $\alpha(1 \rightarrow 3)-\beta-$ GalNAc4,6S

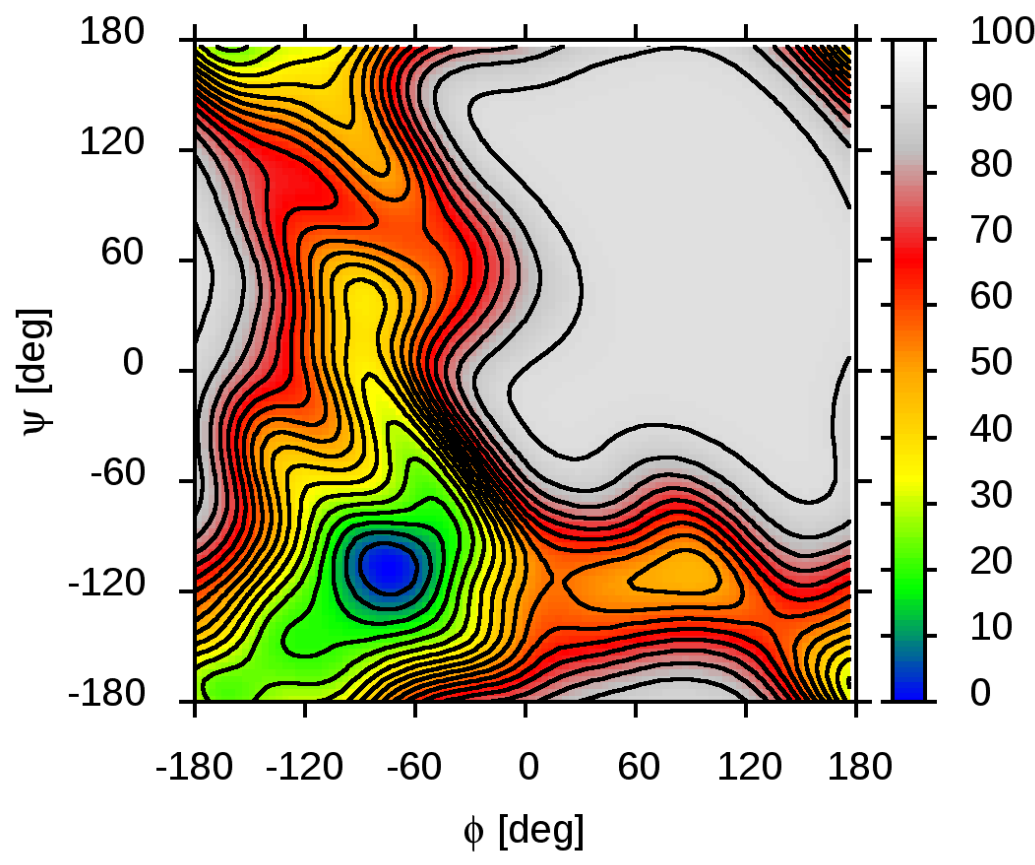

b. GalNAc4,6R ${ }^{1}-\beta(1 \rightarrow 4)-\alpha-L_{-I d o A 2 R^{1}}$

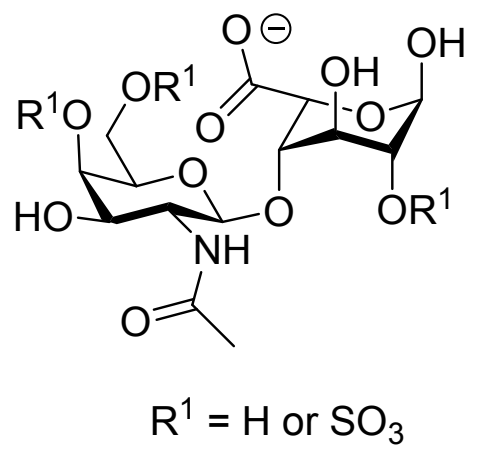


1. GalNAc- $\beta(1 \rightarrow 4)-\alpha-L-I d o A$

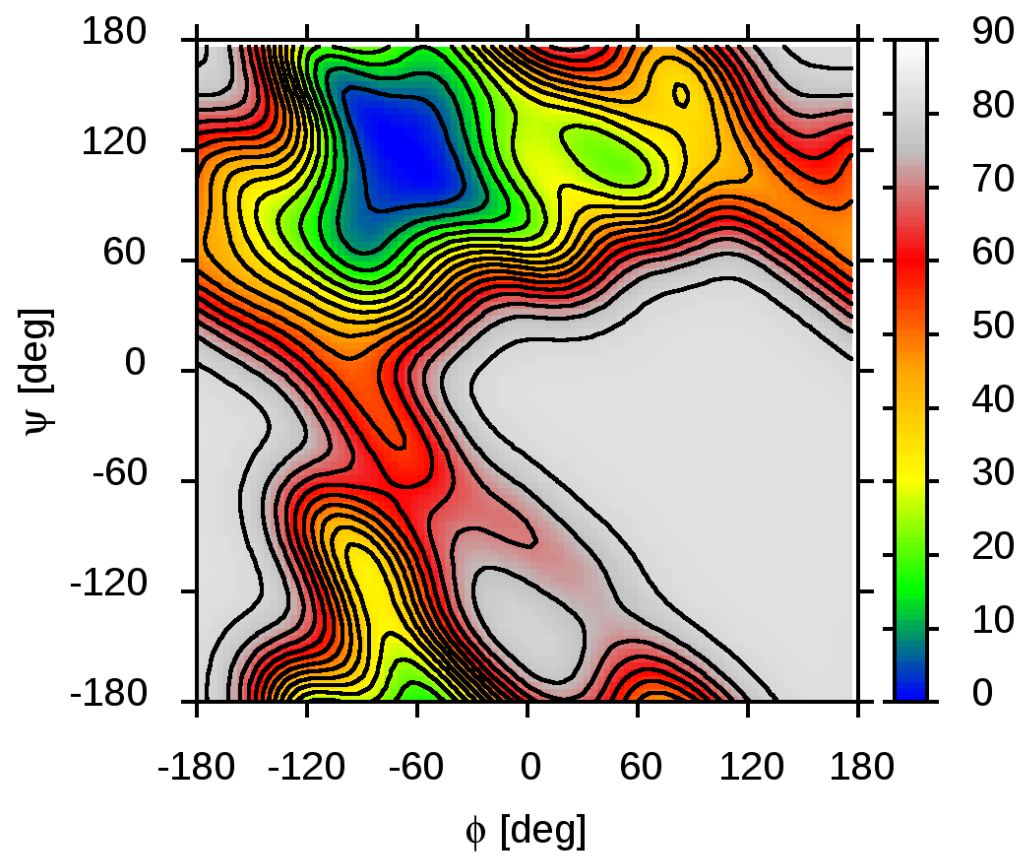

2. GalNAc- $\beta(1 \rightarrow 4)-\alpha-L-I d o A 2 S$

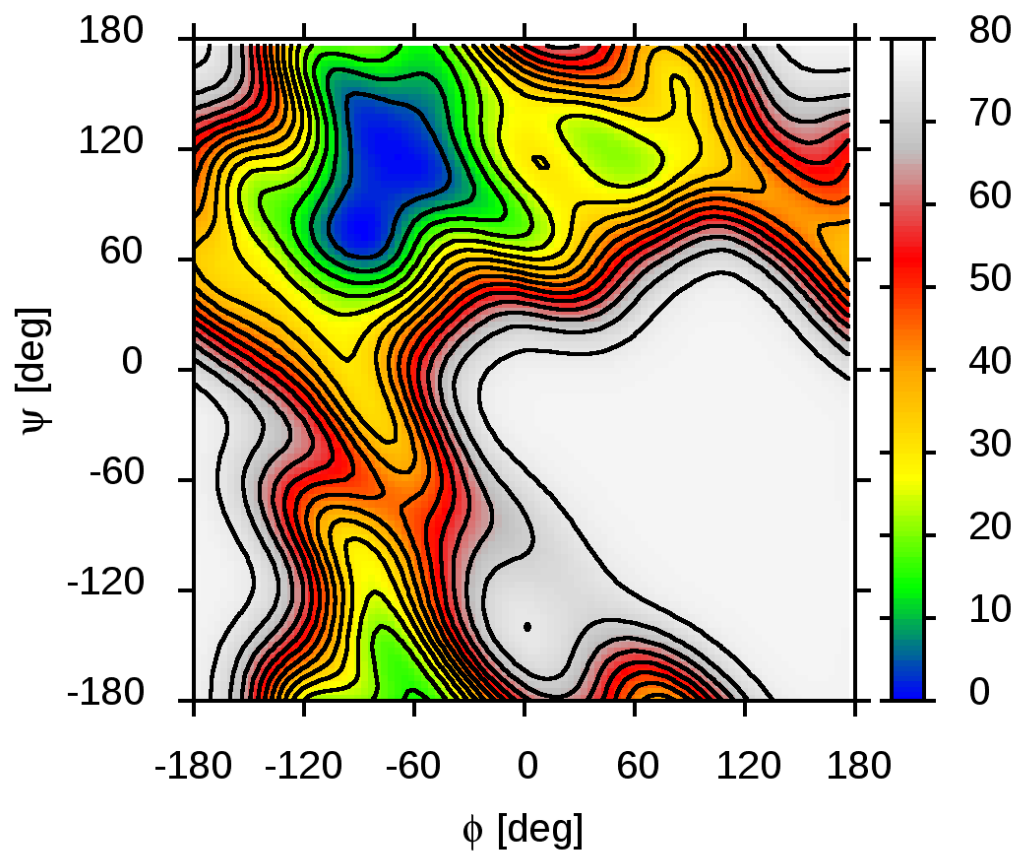


3. GalNAc4S- $\beta(1 \rightarrow 4)-\alpha-L-I d o A$

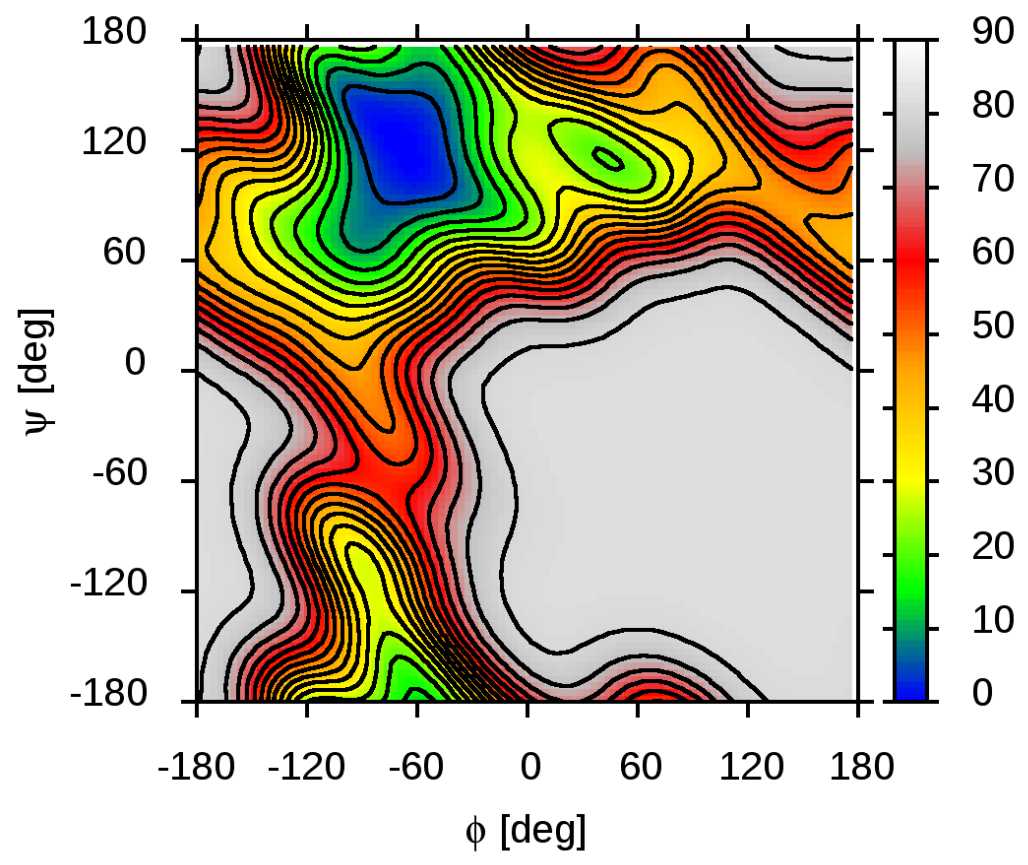

4. GalNAc6S- $\beta(1 \rightarrow 4)-\alpha-L-I d o A$

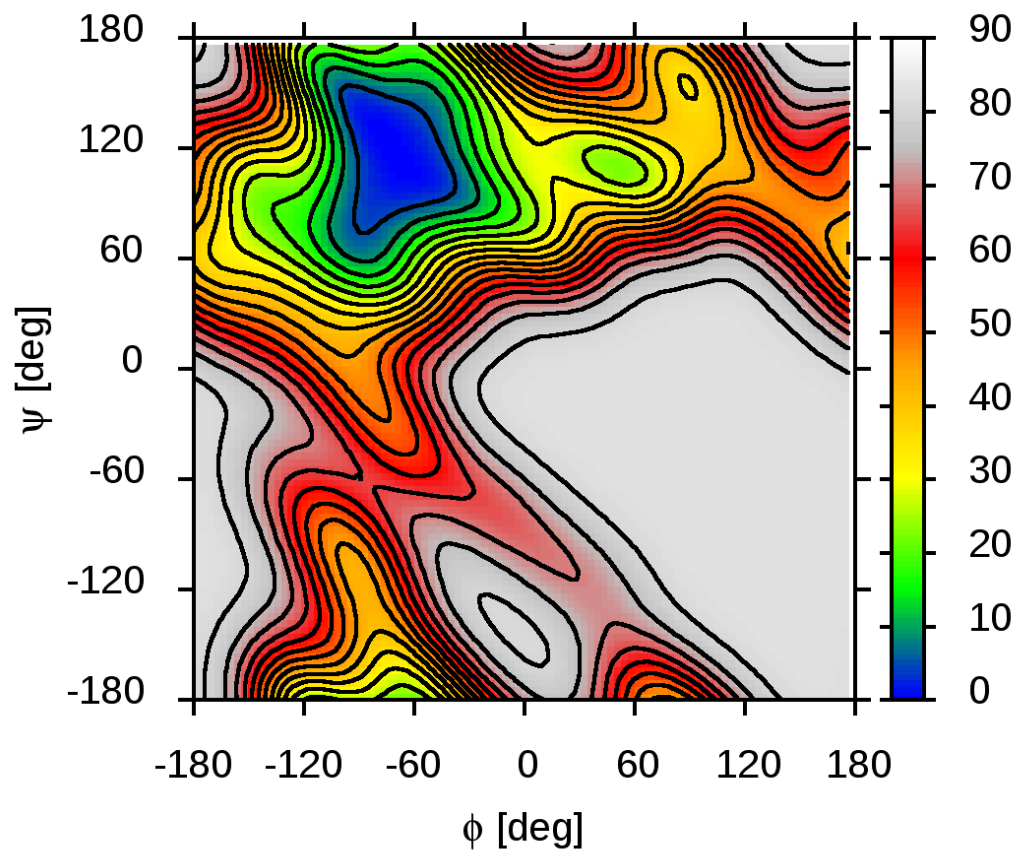


5. GalNAc4S- $\beta(1 \rightarrow 4)-\alpha-$-LIdoA2S

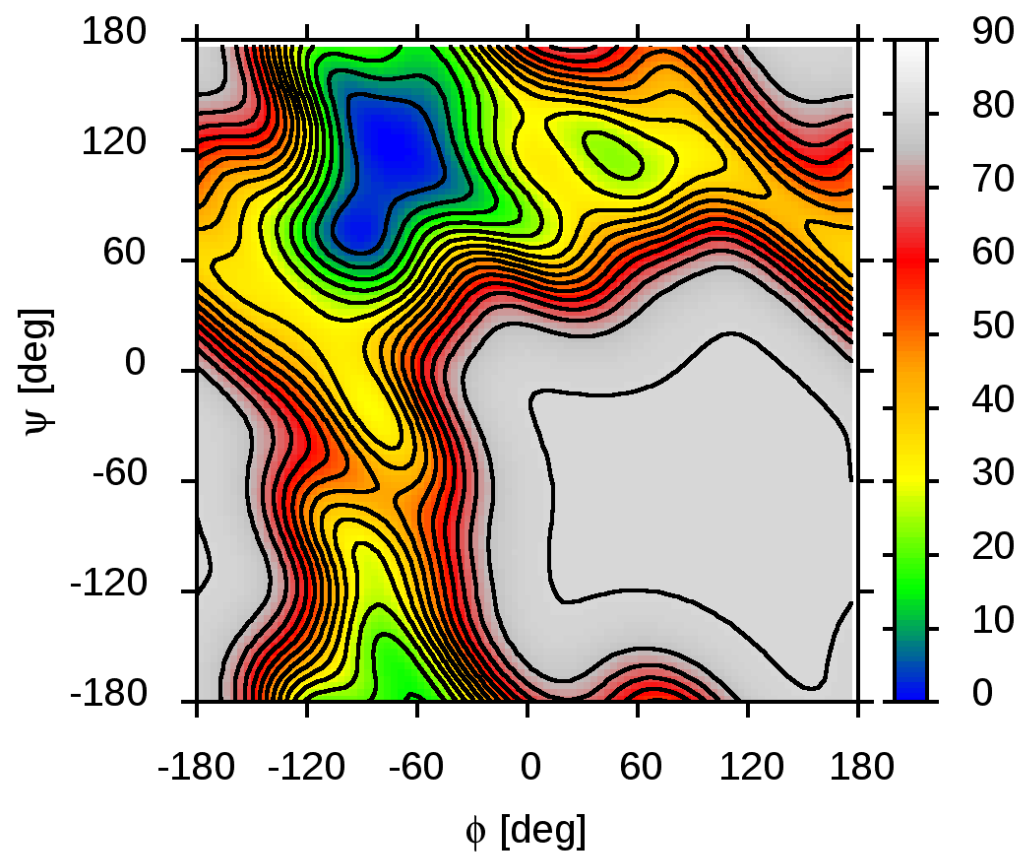

6. GalNAc6S- $\beta(1 \rightarrow 4)-\alpha-L-I d o A 2 S$

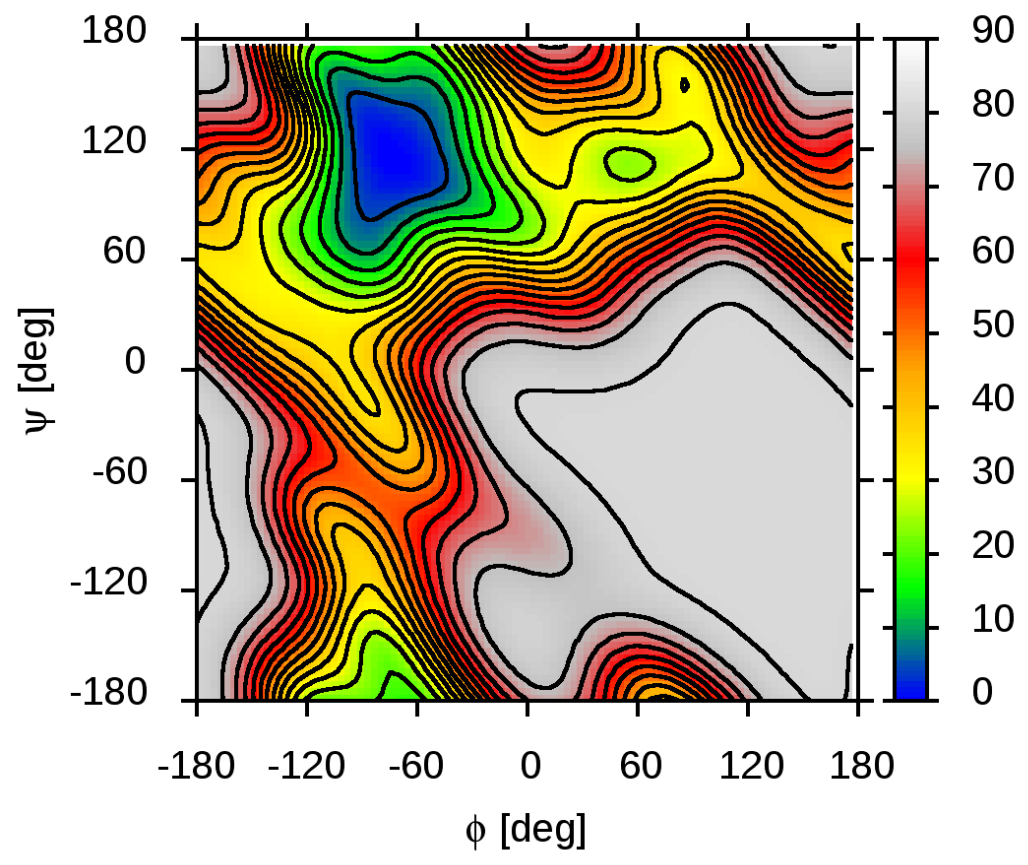


7. GalNAc4,6S- $\beta(1 \rightarrow 4)-\alpha-L-I d o A$

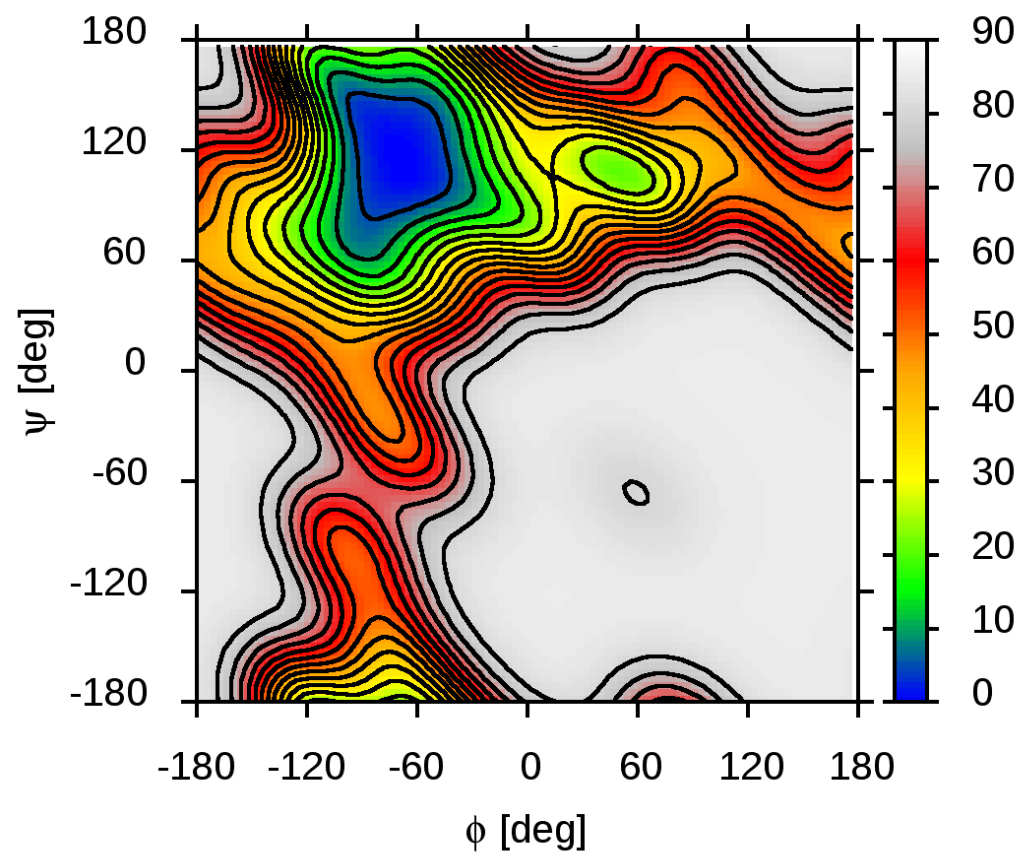

8. GalNAc4,6S- $\beta(1 \rightarrow 4)-\alpha-L-I d o A 2 S$

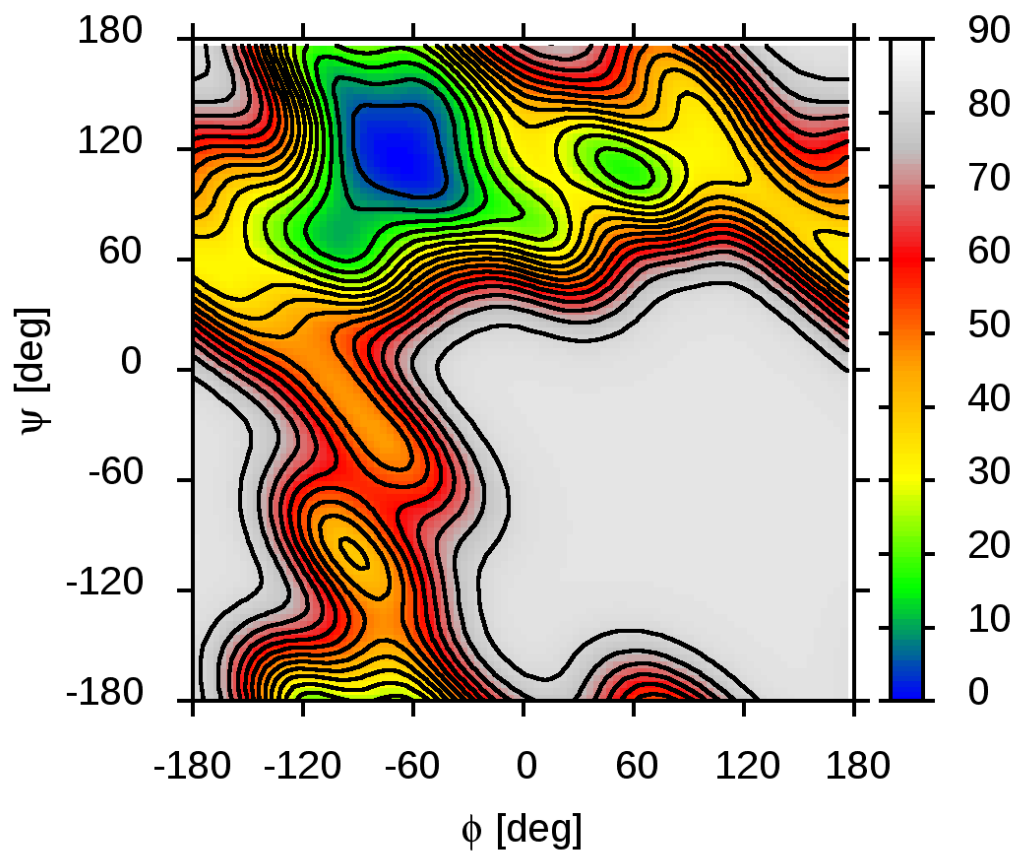


IV. Keratan sulfate (KS)

a. Gal6R ${ }^{1}-\beta(1 \rightarrow 4)-\beta-$ GlcNAc6R $^{1}$

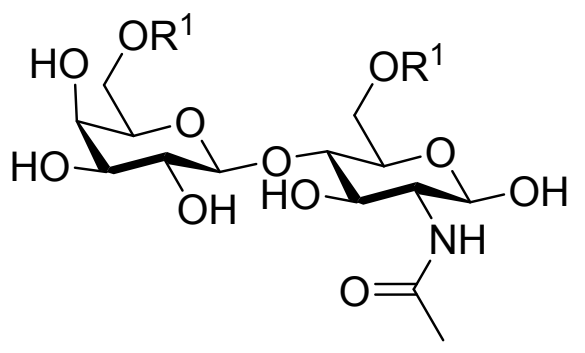

$$
\mathrm{R}^{1}=\mathrm{H} \text { or } \mathrm{SO}_{3}
$$

1. Gal- $\beta(1 \rightarrow 4)-\beta-G l c N A c$

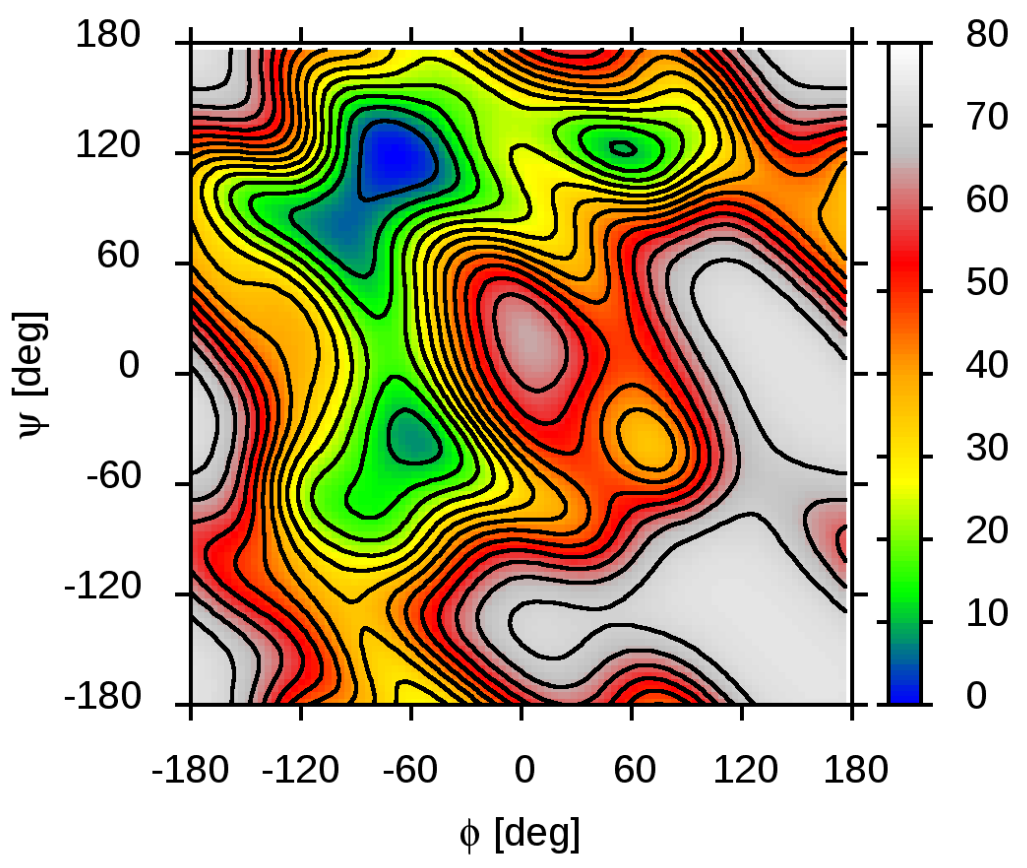


2. Gal- $\beta(1 \rightarrow 4)-\beta-$ GlcNAc6S

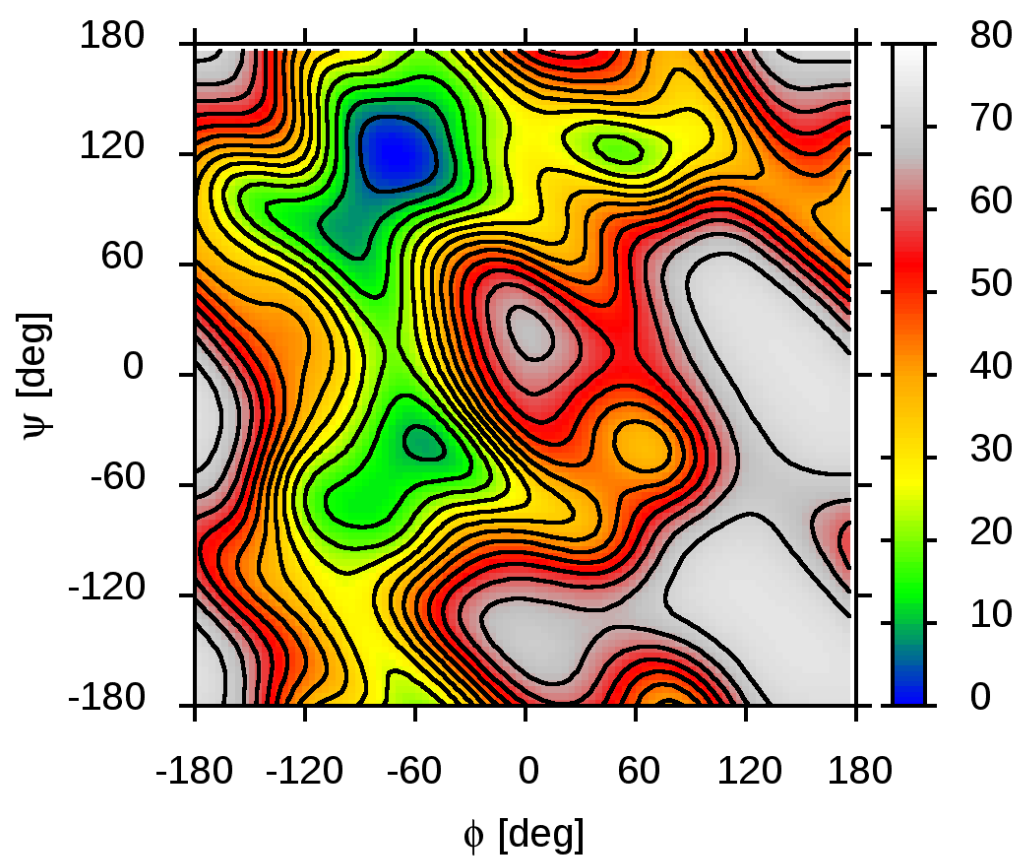

3. Gal6S- $\beta(1 \rightarrow 4)-\beta-$ GlcNAc

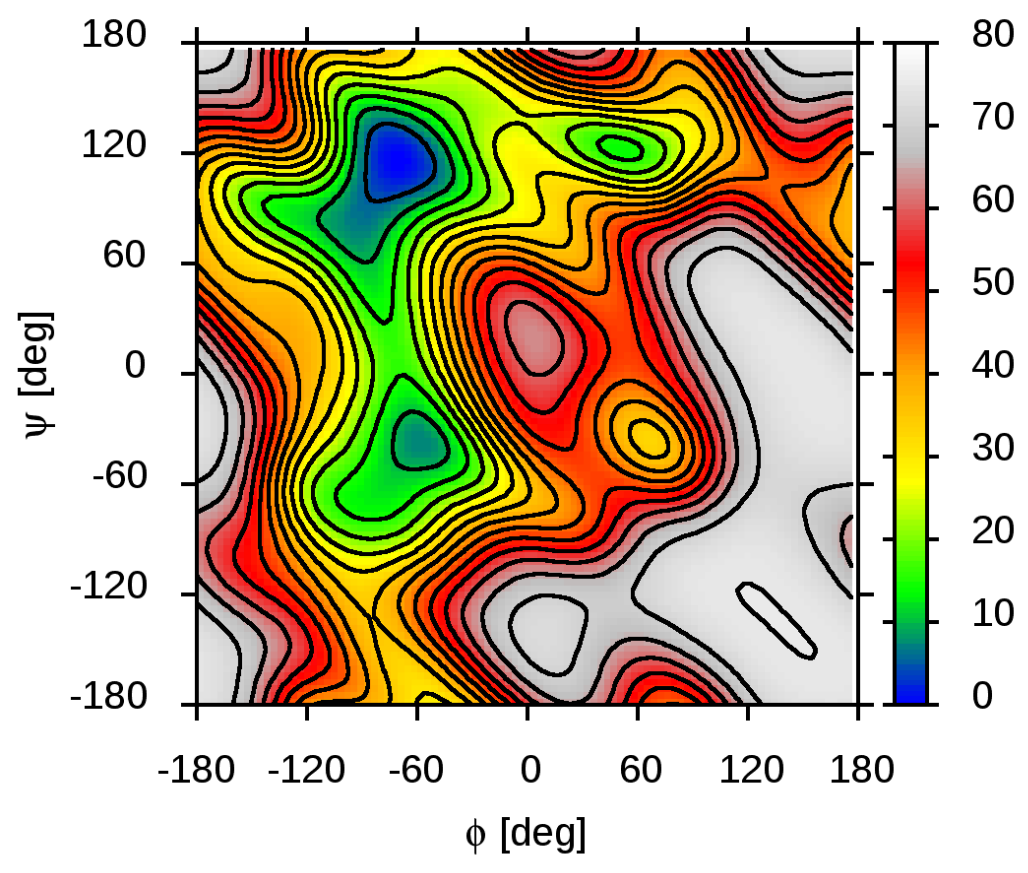


4. Gal6S- $\beta(1 \rightarrow 4)-\beta-$-GlcNAc6S

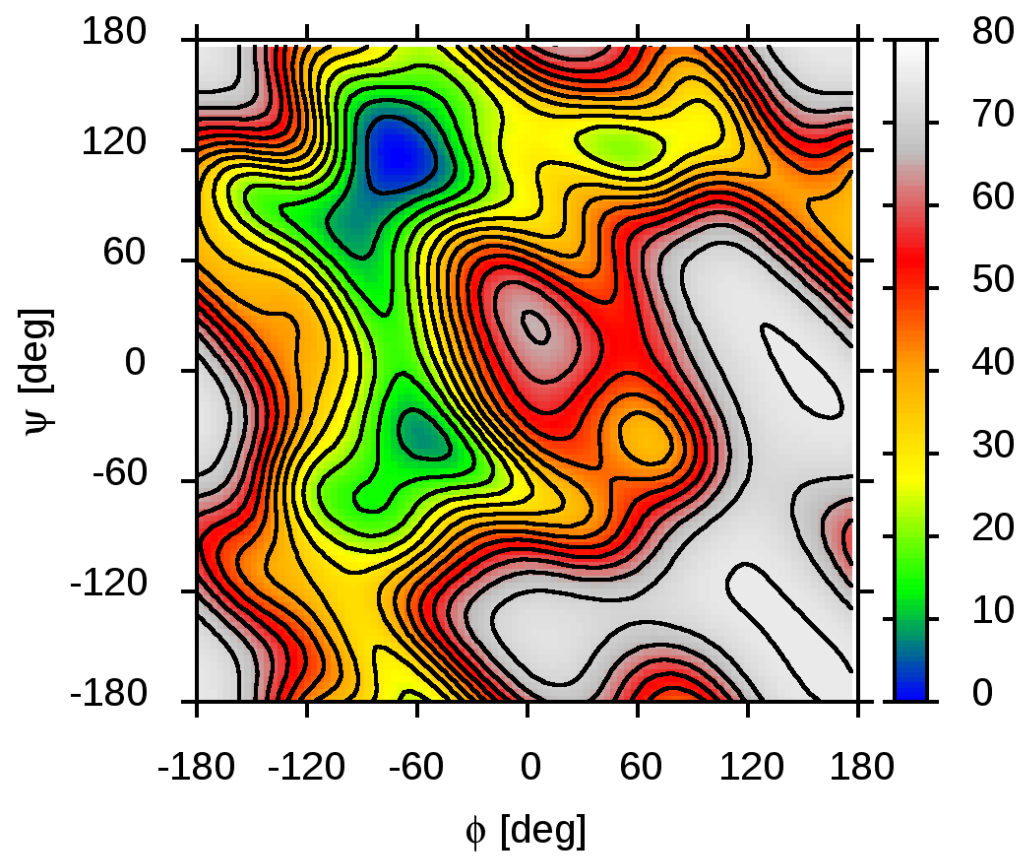

b. GlcNAc6R ${ }^{1}-\beta(1 \rightarrow 3)-\beta-G^{-} 6 R^{1}$

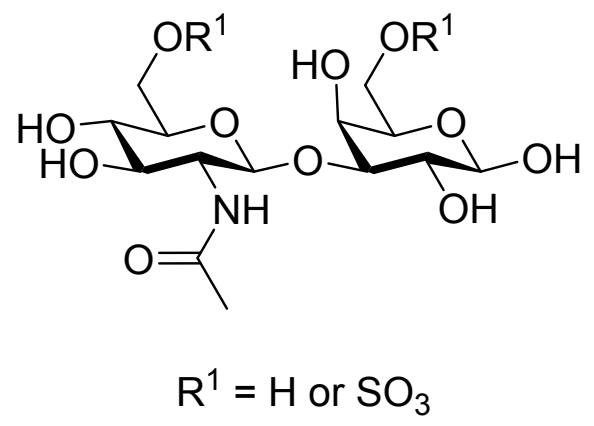


1. GlcNAc- $\beta(1 \rightarrow 3)-\beta-$ Gal

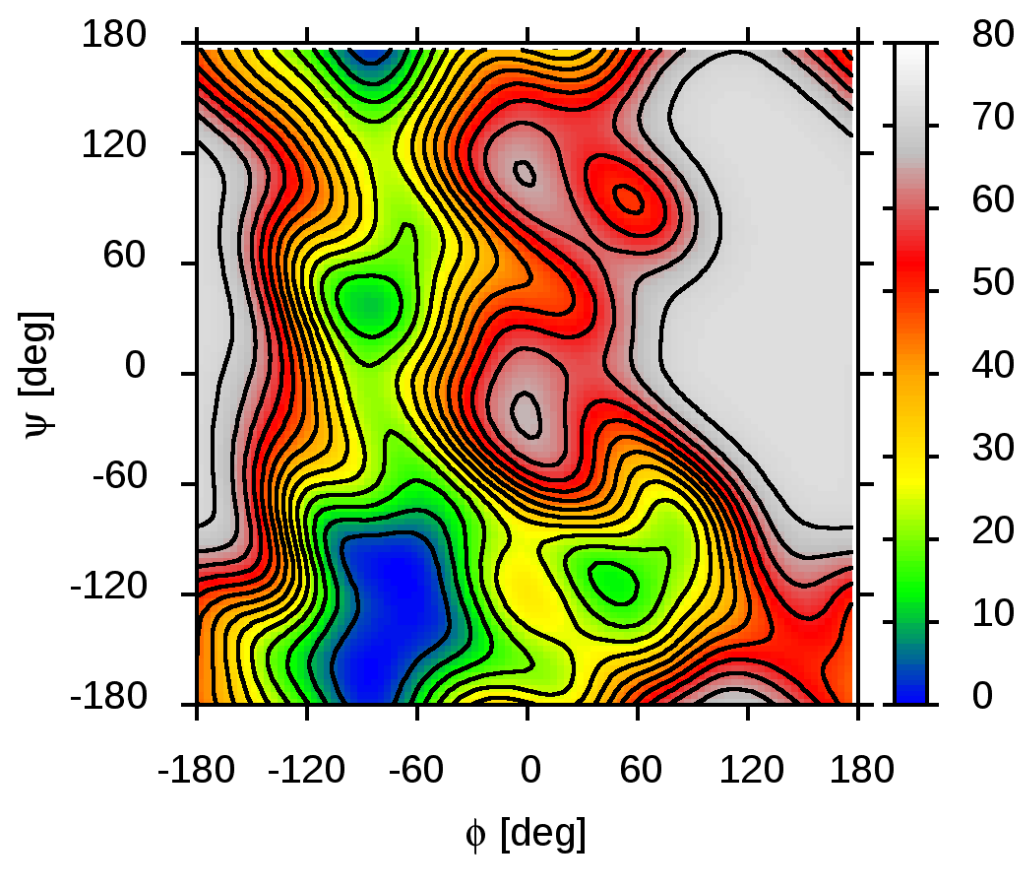

2. GlcNAc- $\beta(1 \rightarrow 3)-\beta-$ Gal6S

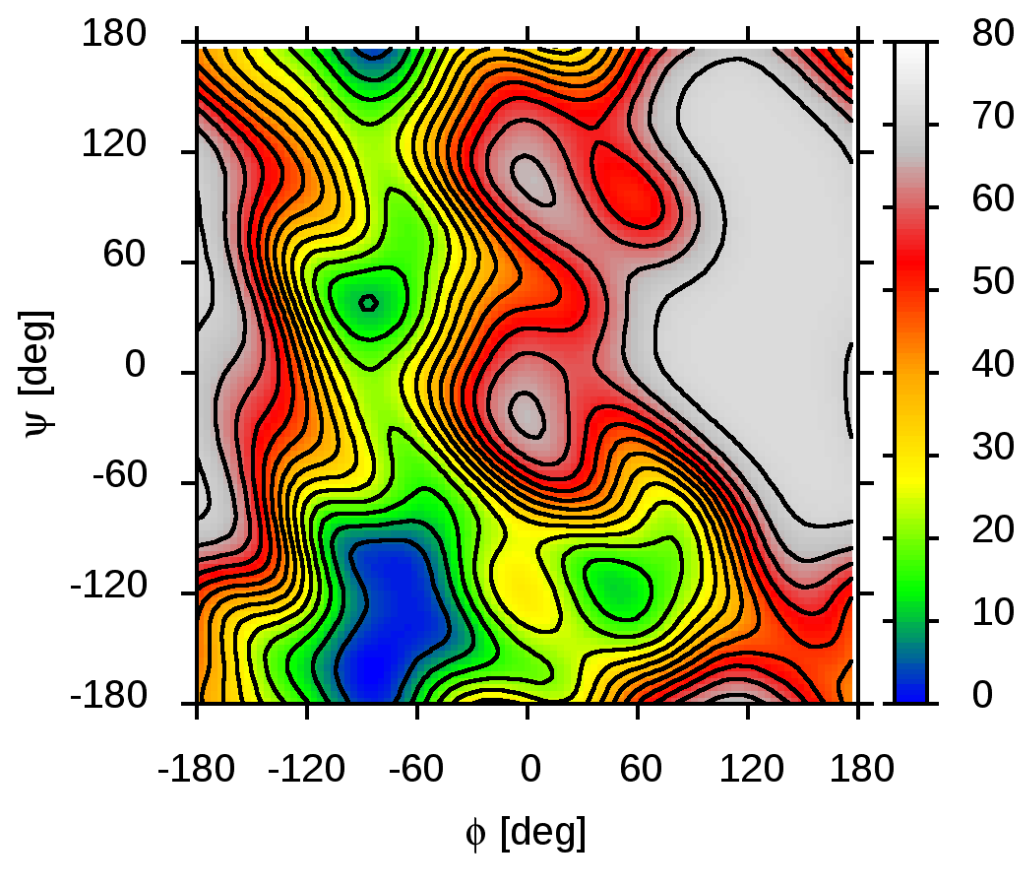


3. GlcNAc6S- $\beta(1 \rightarrow 3)-\beta-$ Gal

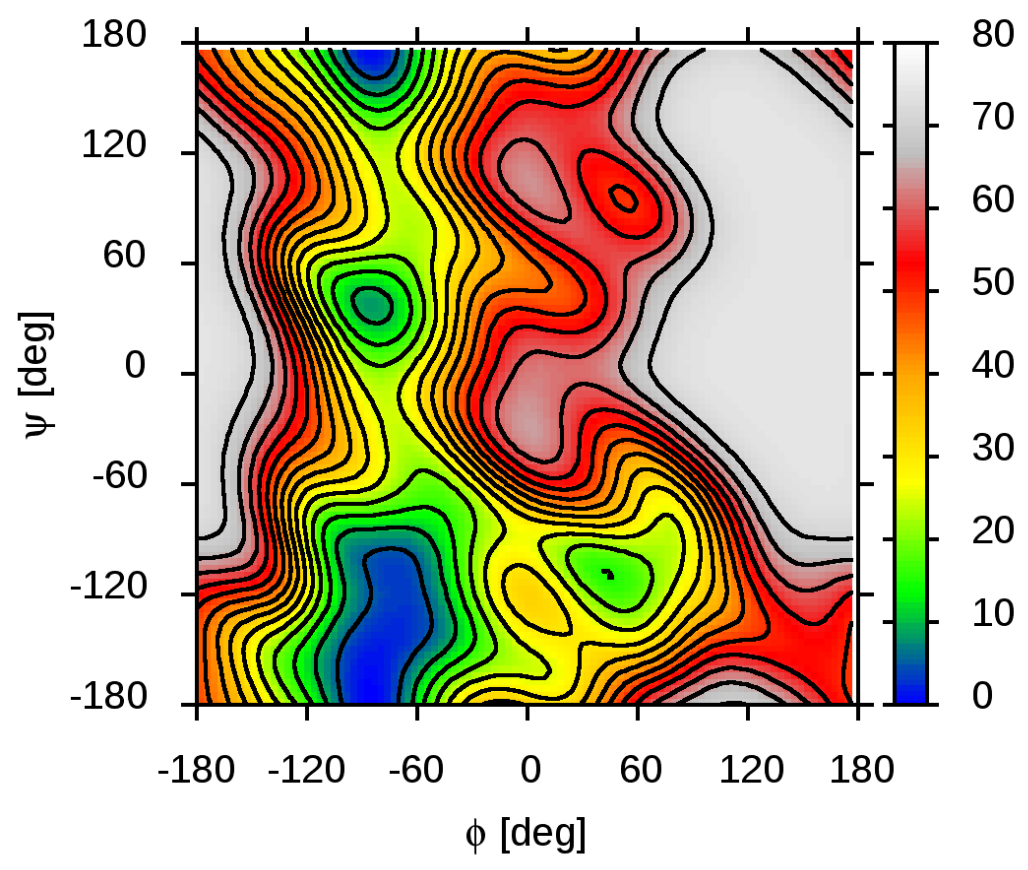

4. GlcNAc6S- $\beta(1 \rightarrow 3)-\beta-$-Gal6S

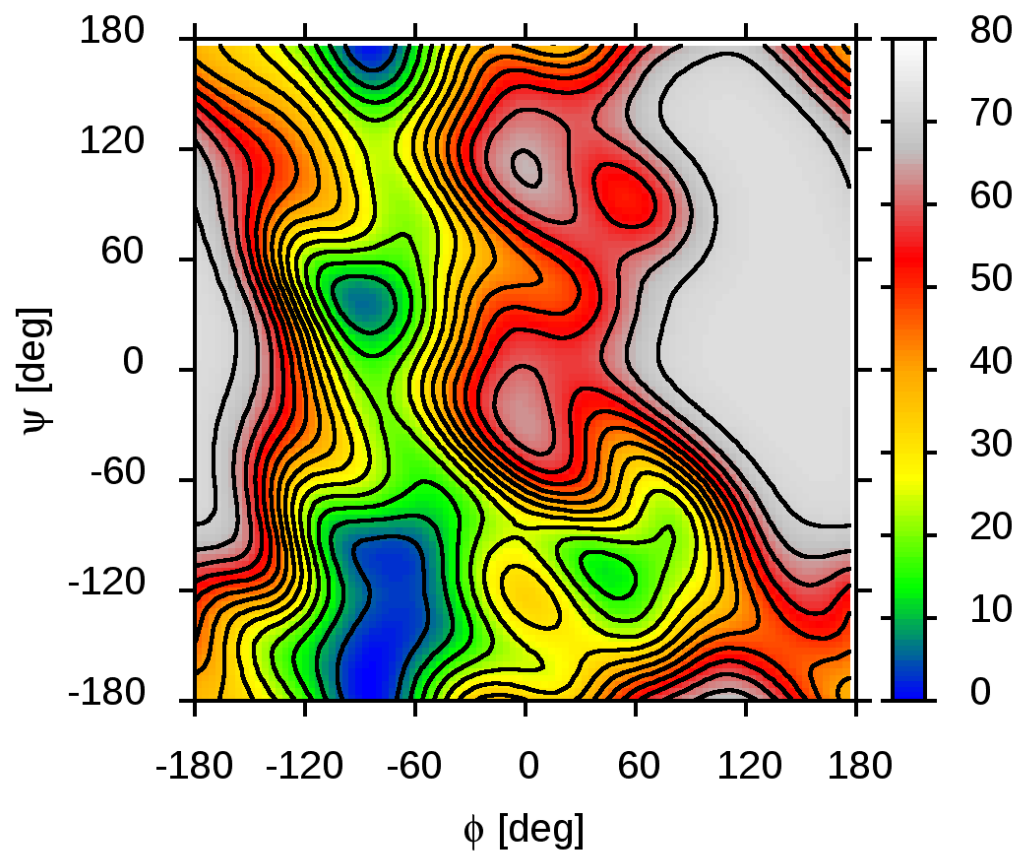


V. Heparin/Heparan sulfate (HP/HS)

a. L-IdoA2R ${ }^{1}-\alpha(1 \rightarrow 4)-\alpha-G l c N R^{2} 3,6 R^{1}$

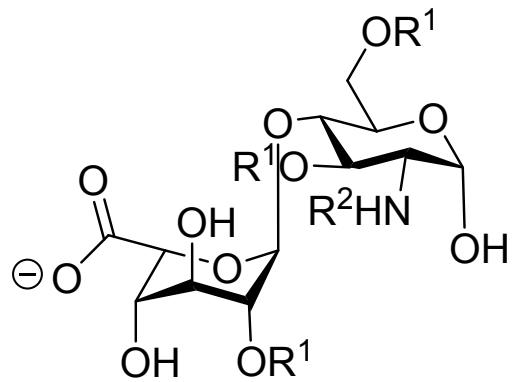

$$
\begin{aligned}
& \mathrm{R}^{1}=\mathrm{H} \text { or } \mathrm{SO}_{3} \\
& \mathrm{R}^{2}=\mathrm{Ac} \text { or } \mathrm{SO}_{3}
\end{aligned}
$$

1. L-IdoA- $\alpha(1 \rightarrow 4)-\alpha-G \mid c N A c$

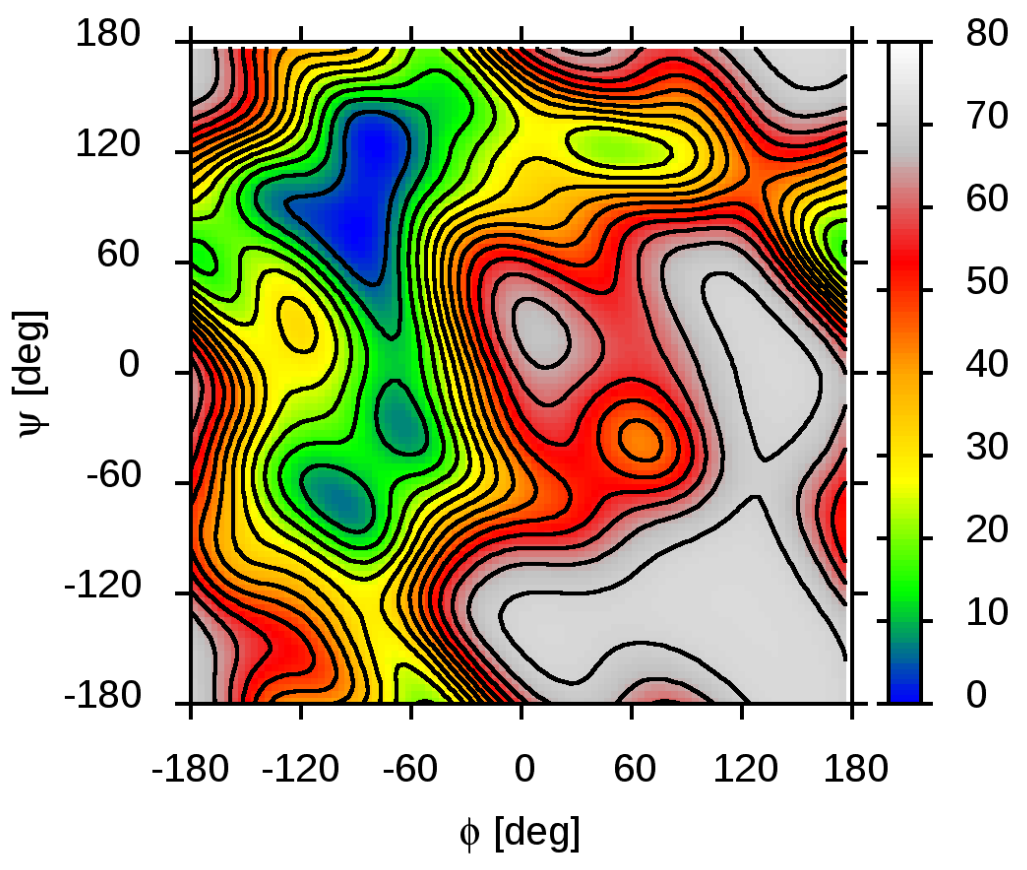


2. L-IdoA- $\alpha(1 \rightarrow 4)-\alpha-$ GlcNAc3S

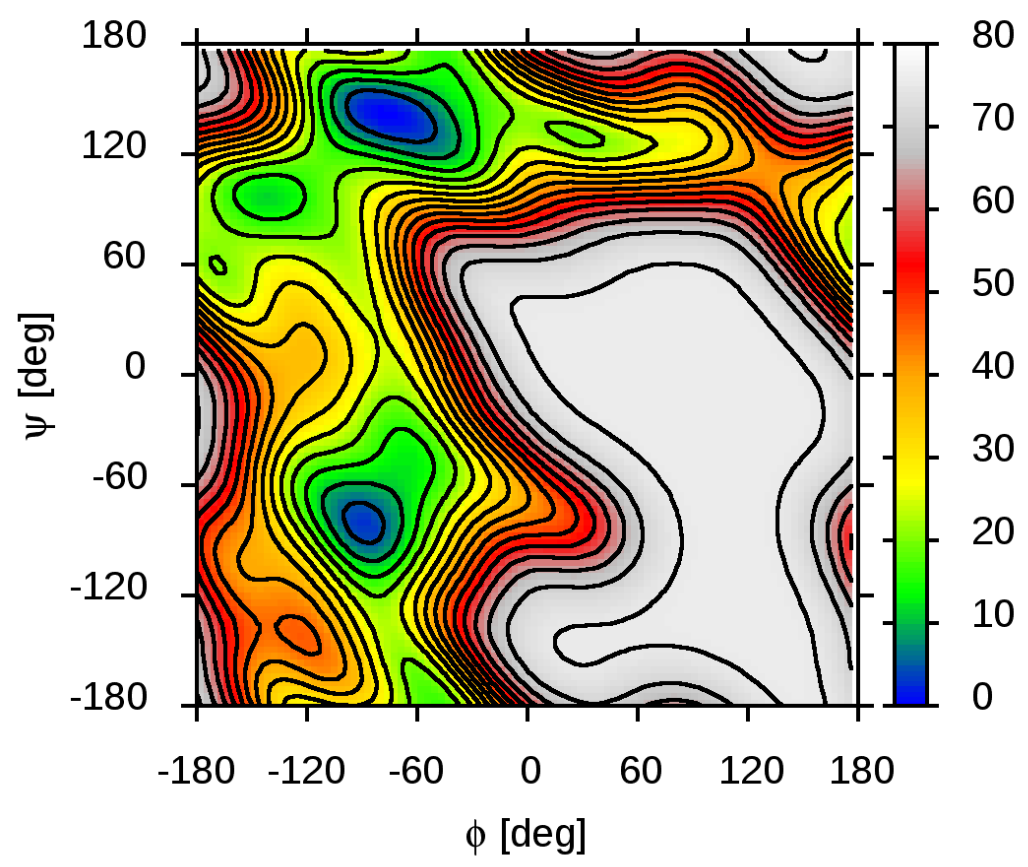

3. L-IdoA- $\alpha(1 \rightarrow 4)-\alpha-G \mid c N A c 6 S$

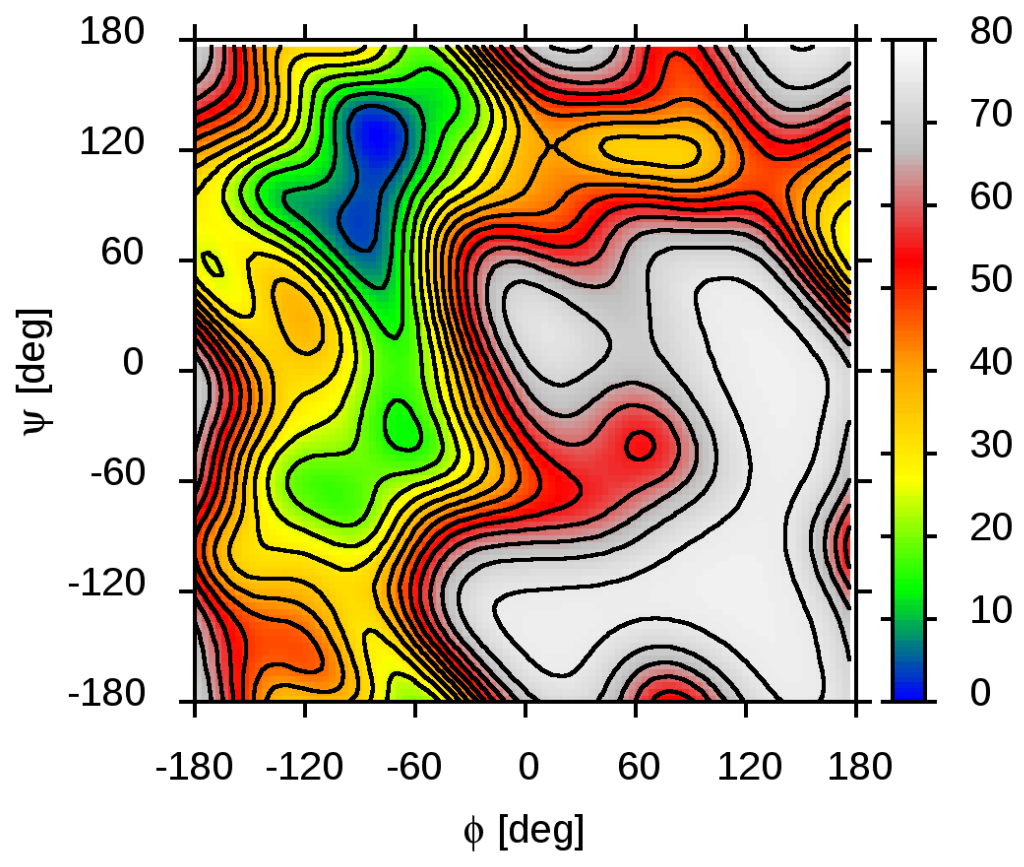


4. L-IdoA2S- $\alpha(1 \rightarrow 4)-\alpha-$ GlcNAc

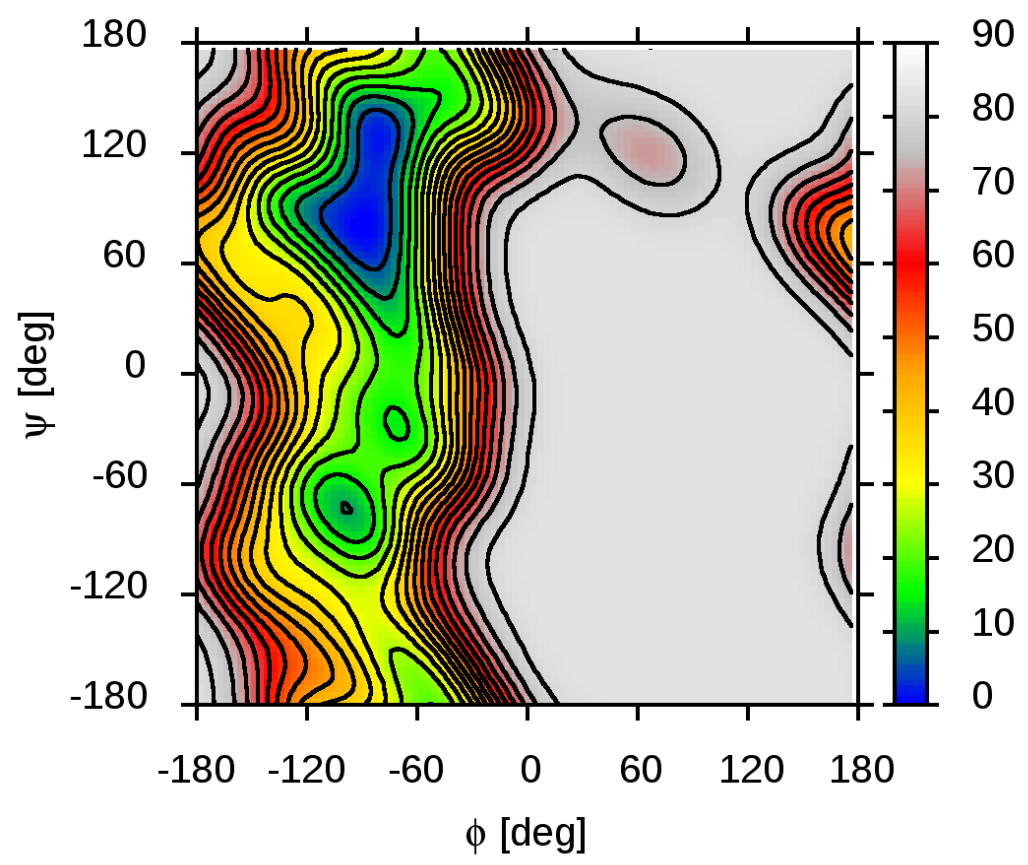

5. L-IdoA2S- $\alpha(1 \rightarrow 4)-\alpha-G l c N A c 3 S$

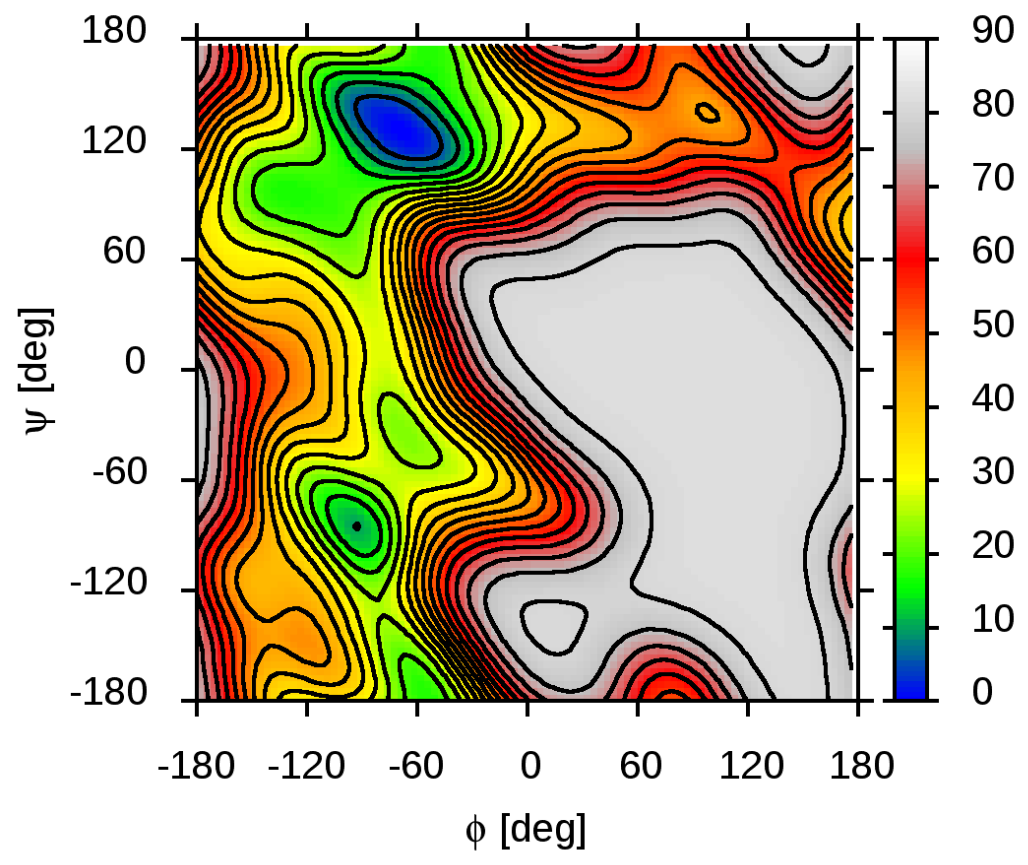


6. L-IdoA2S- $\alpha(1 \rightarrow 4)-\alpha-$ GIcNAc6S

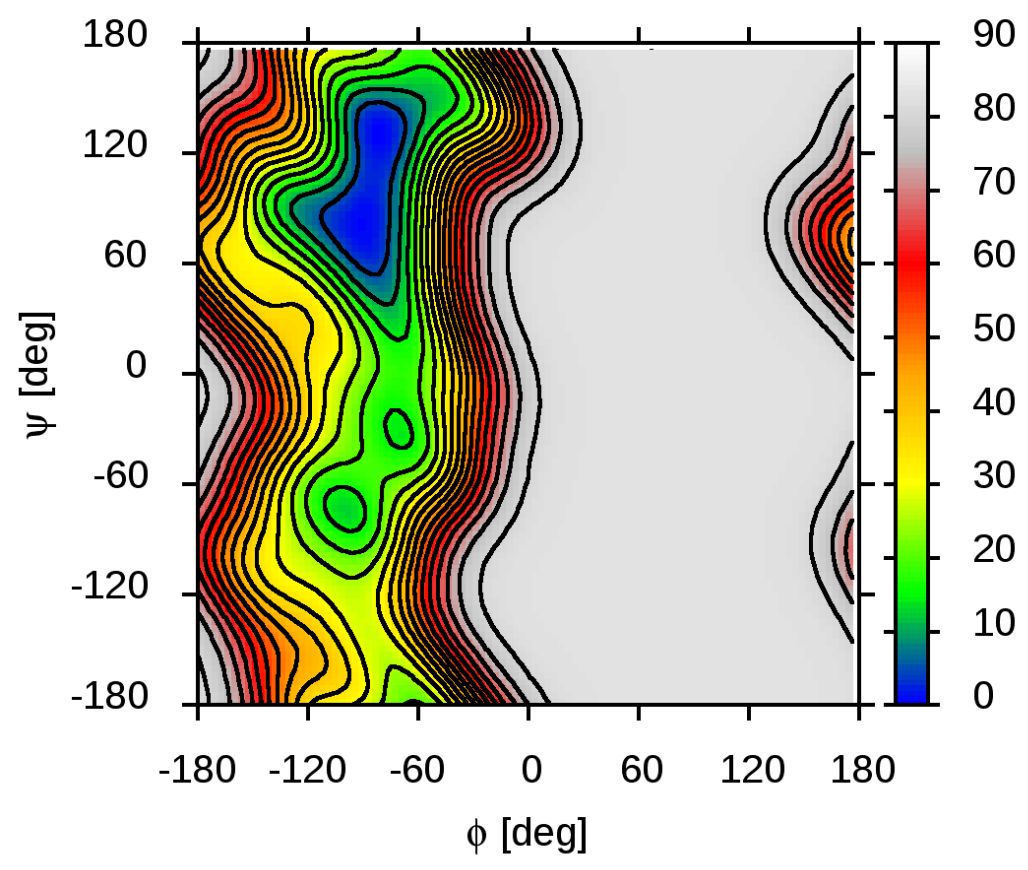

7. L-IdoA- $\alpha(1 \rightarrow 4)-\alpha-G \mid c N A c 3,6 S$

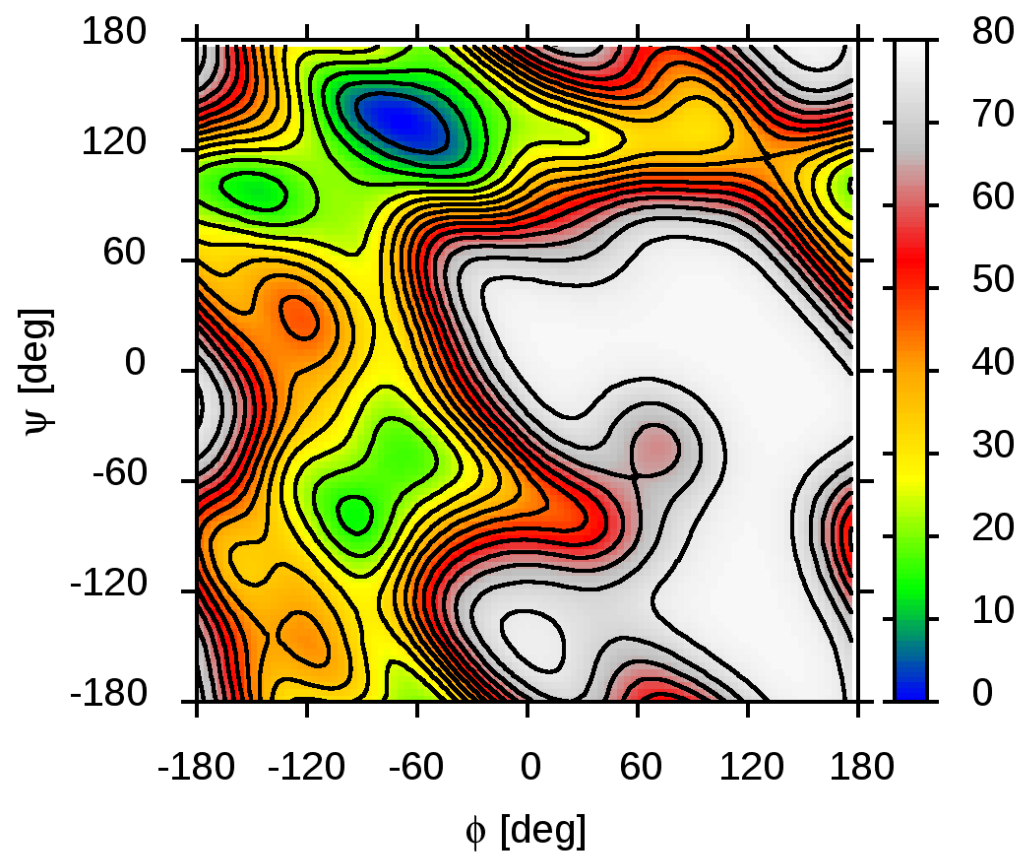


8. L-IdoA2S- $\alpha(1 \rightarrow 4)-\alpha-G l c N A c 3,6 S$

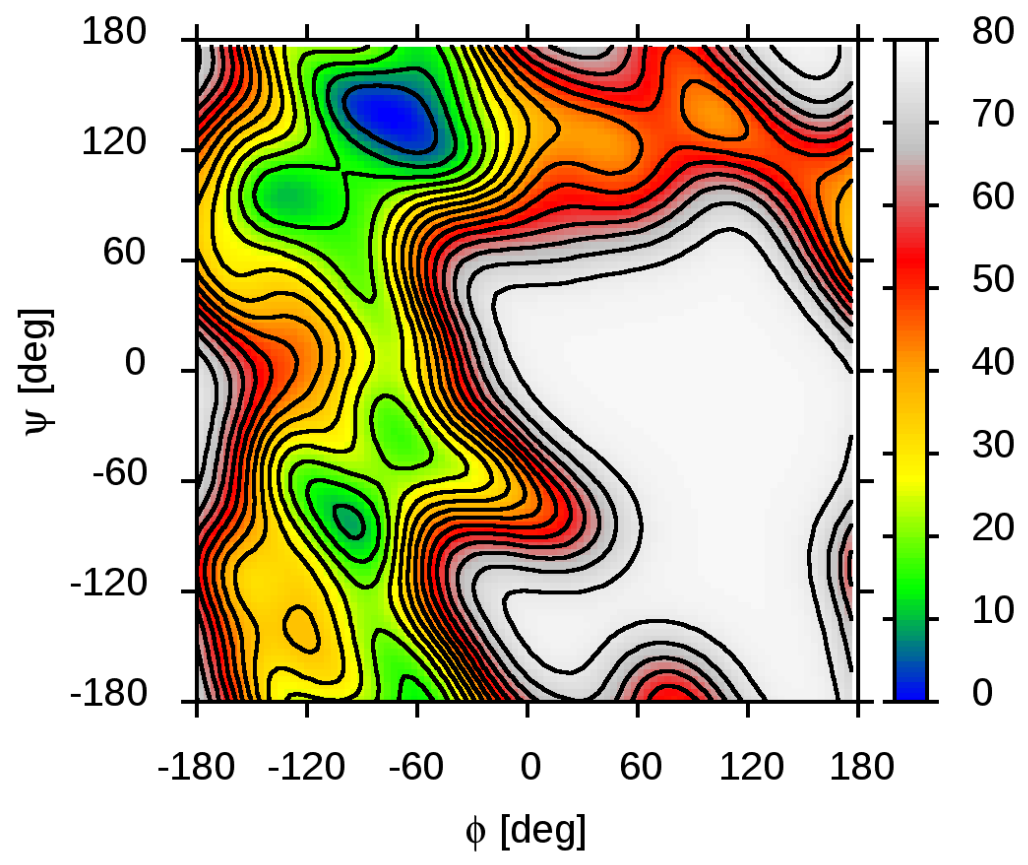

9. L-IdoA- $\alpha(1 \rightarrow 4)-\alpha-$ GICNS

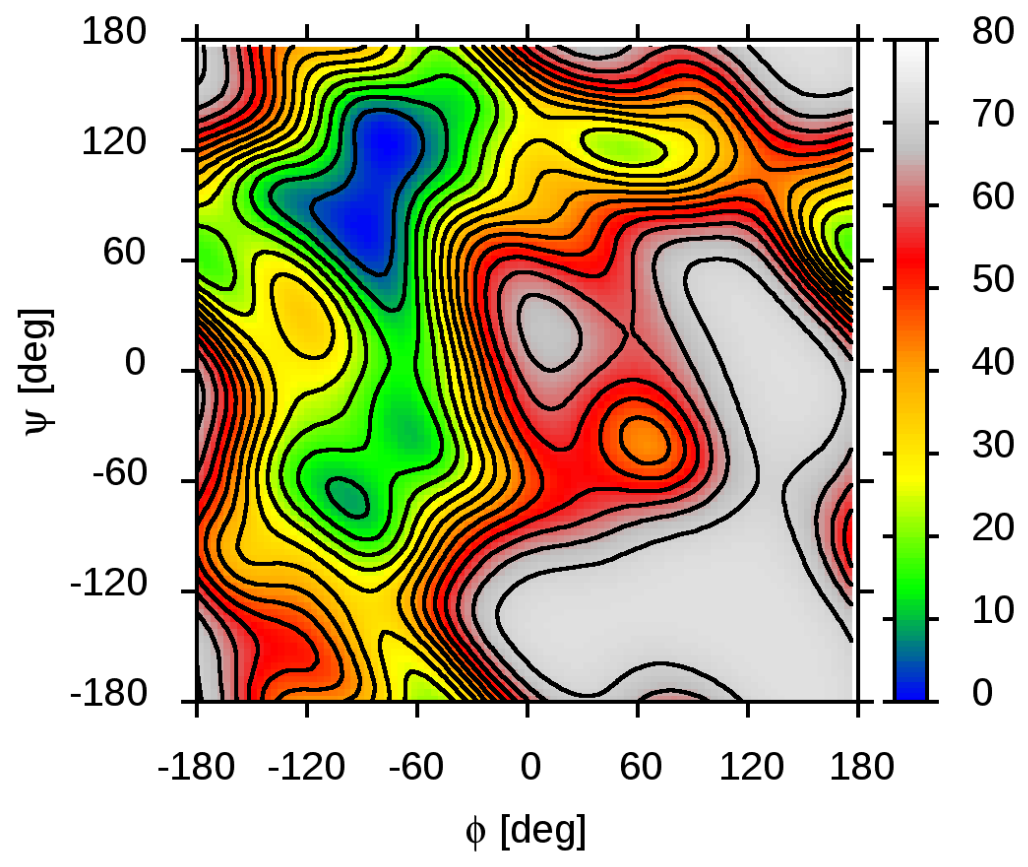


10. L-IdoA- $\alpha(1 \rightarrow 4)-\alpha-$ GICNS3S

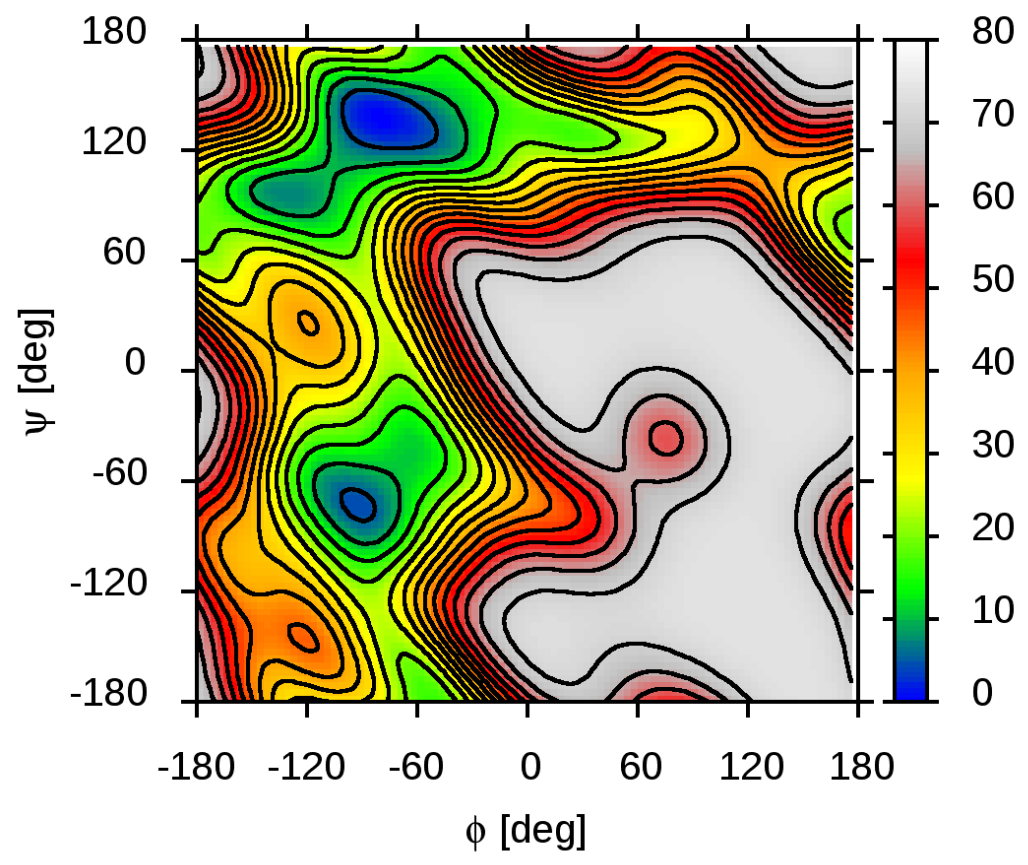

11. L-IdoA- $\alpha(1 \rightarrow 4)-\alpha-G I c N S 6 S$

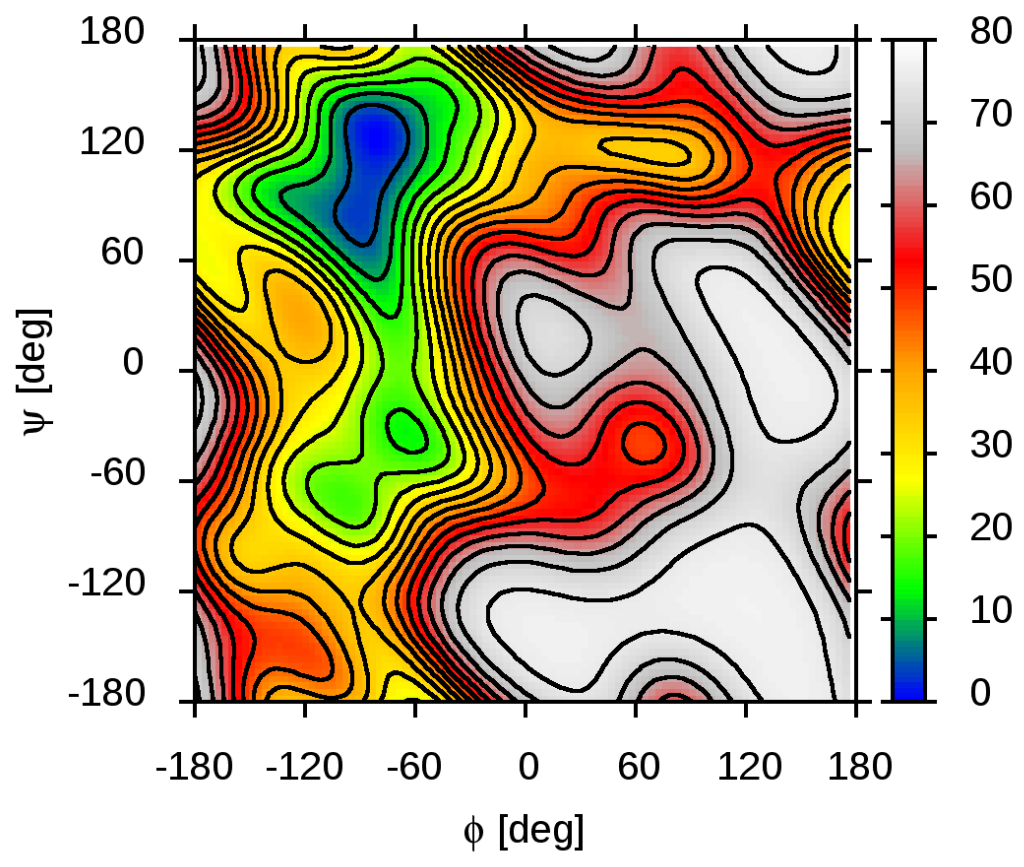


12. L-IdoA2S- $\alpha(1 \rightarrow 4)-\alpha-G I c N S$

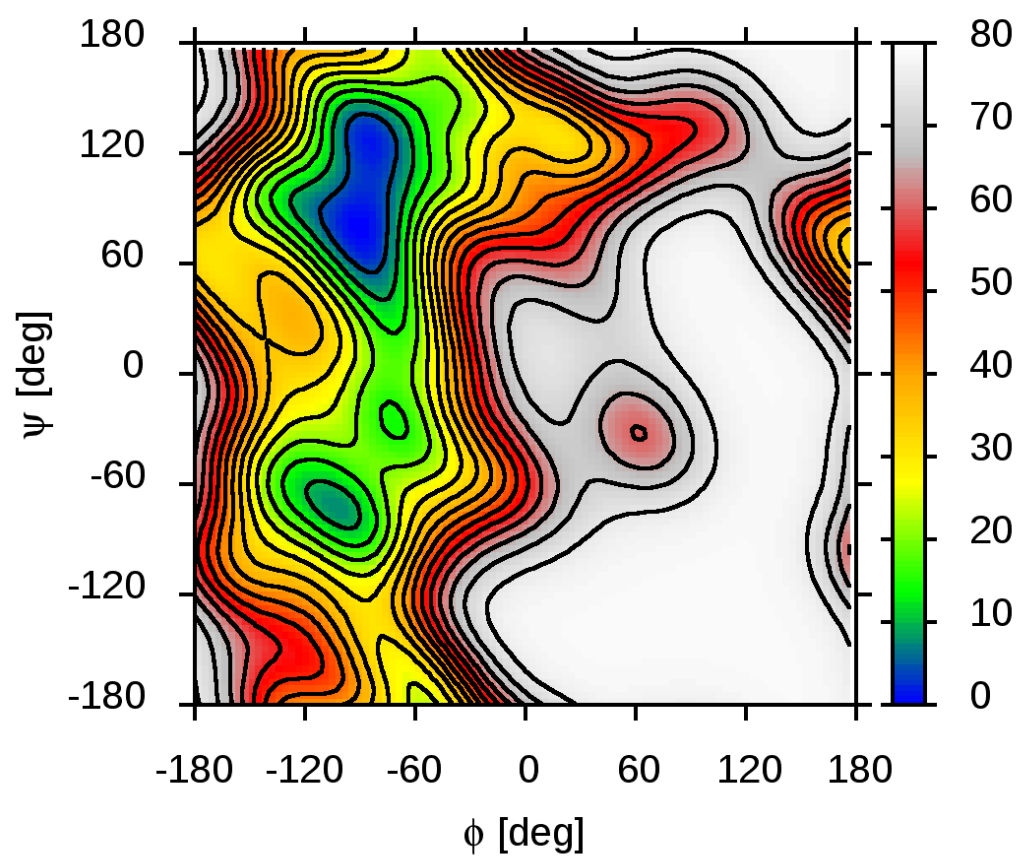

13. L-IdoA2S- $\alpha(1 \rightarrow 4)-\alpha-G I c N S 3 S$

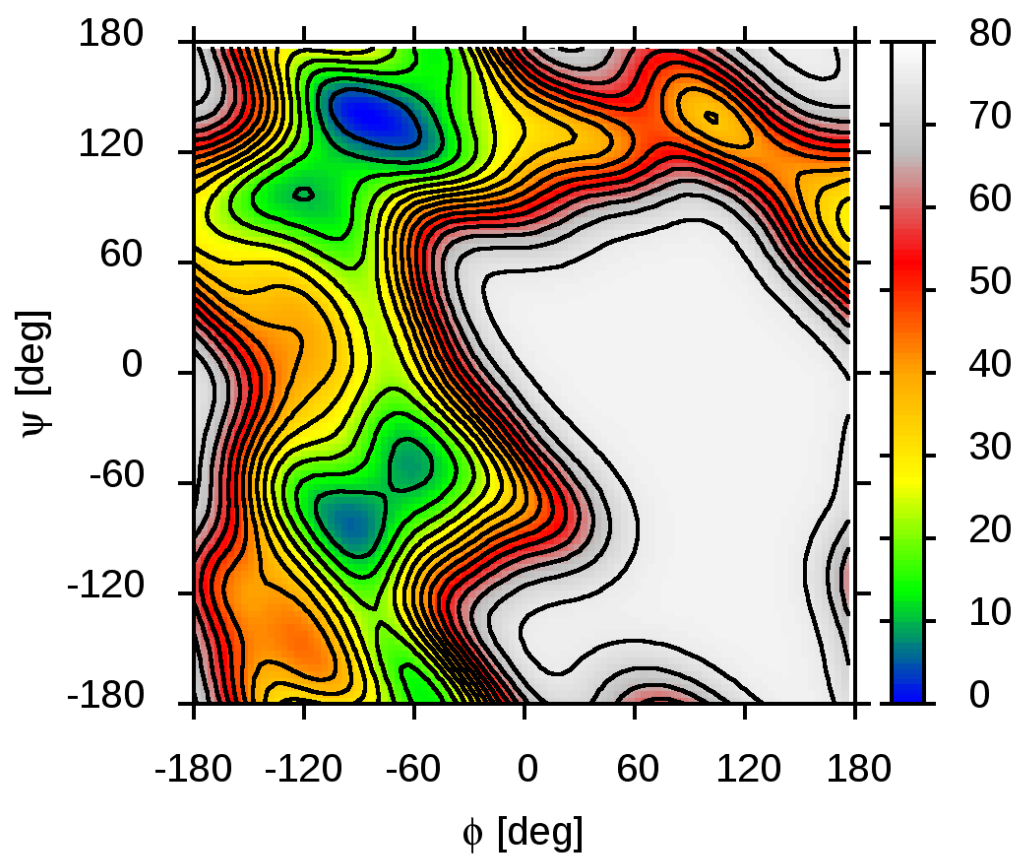


14. L-IdoA2S- $\alpha(1 \rightarrow 4)-\alpha-$-GICNS6S

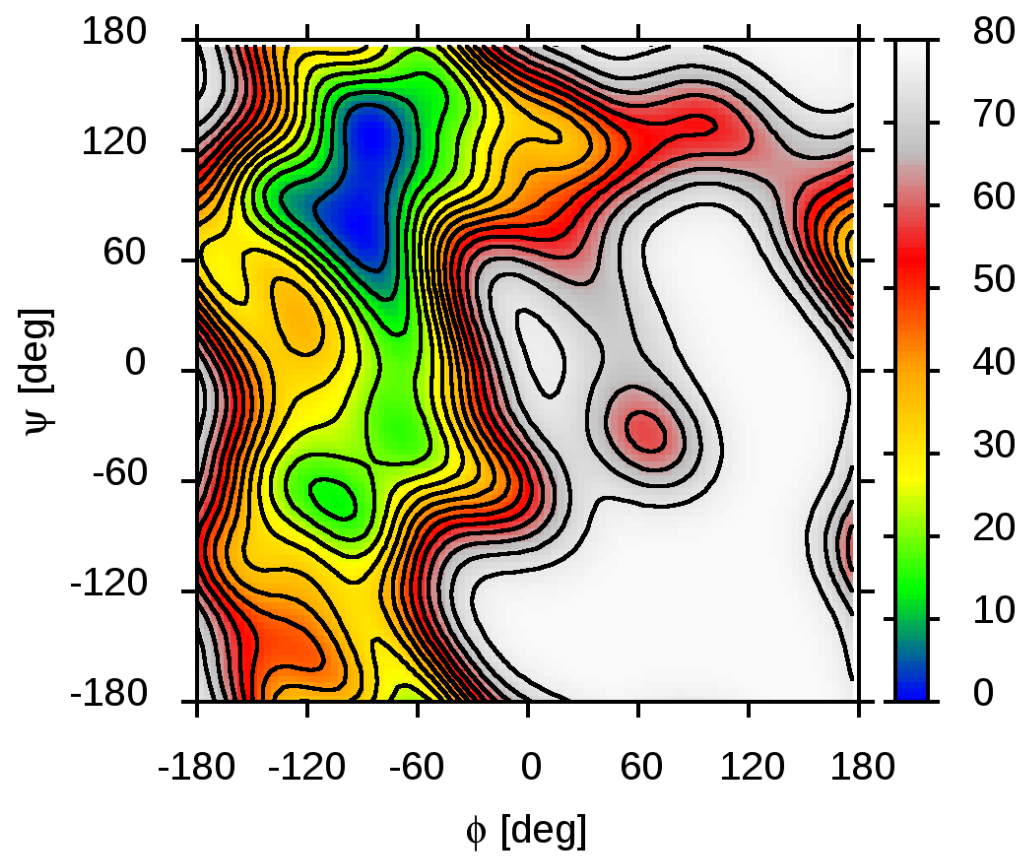

15. L-IdoA- $\alpha(1 \rightarrow 4)-\alpha-$ GIcNS3,6S

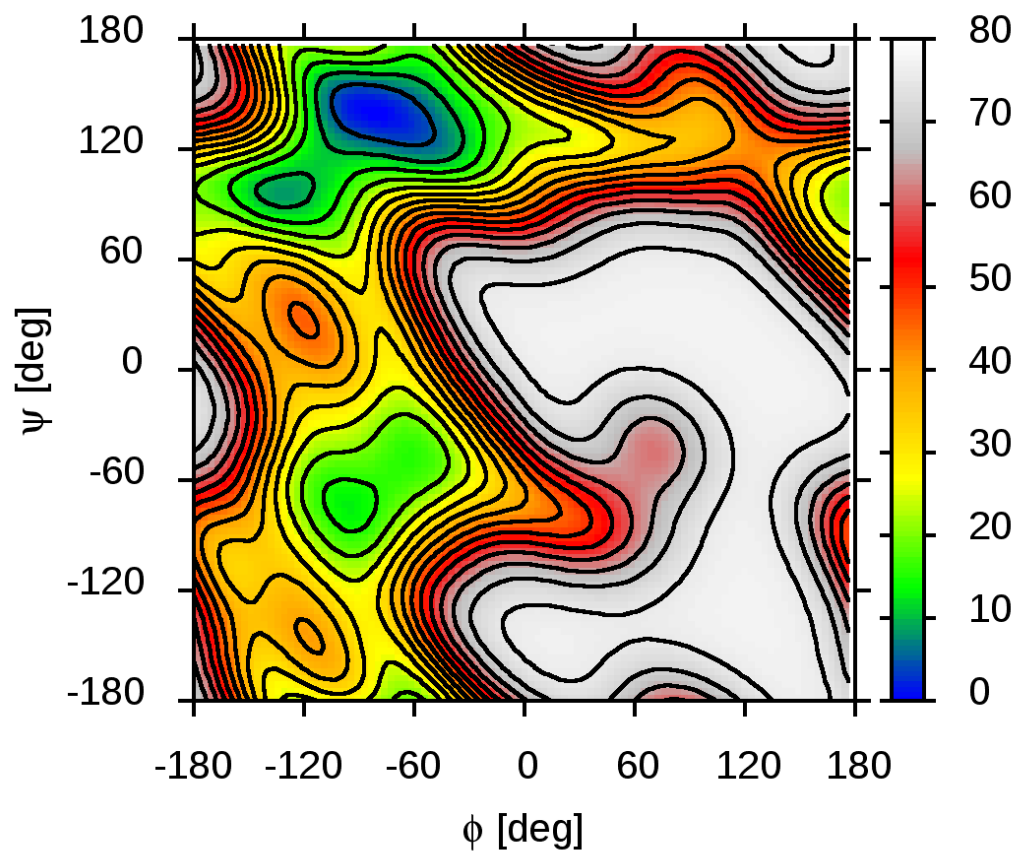


16. L-IdoA2S- $\alpha(1 \rightarrow 4)-\alpha-G I c N S 3,6 S$

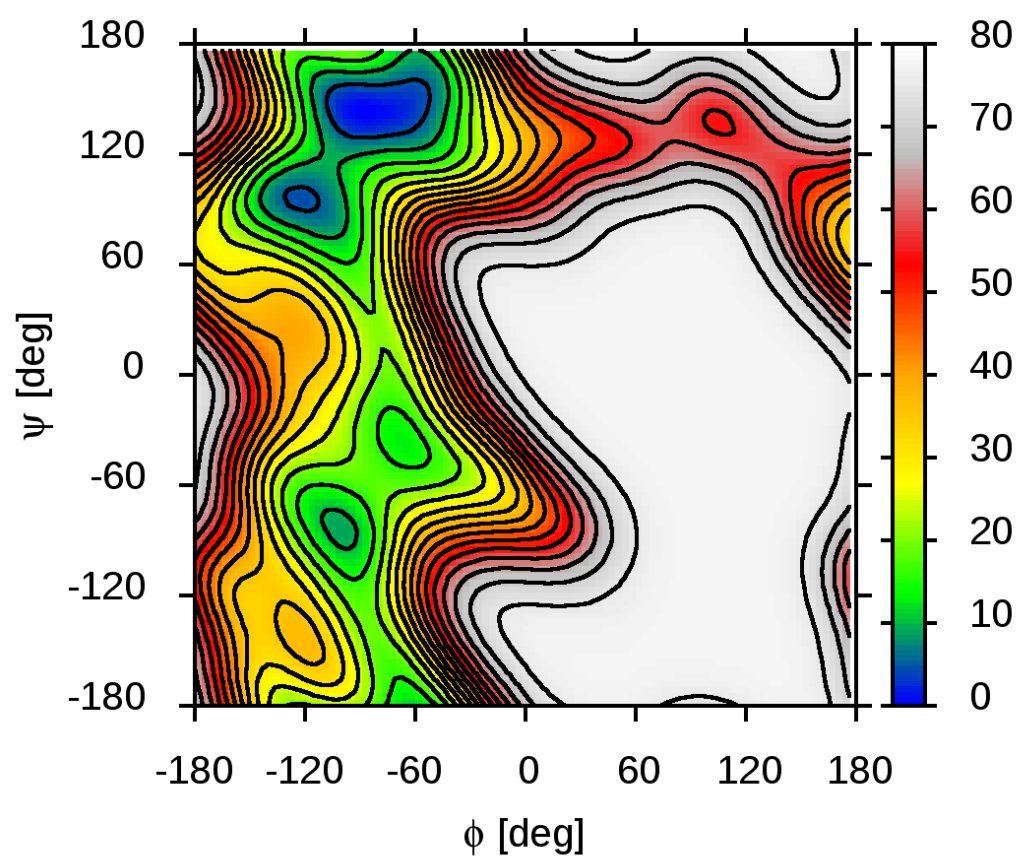

b. GlcNR $23,6 R^{1}-\alpha(1 \rightarrow 4)-\alpha-L-I d o A 2 R^{1}$

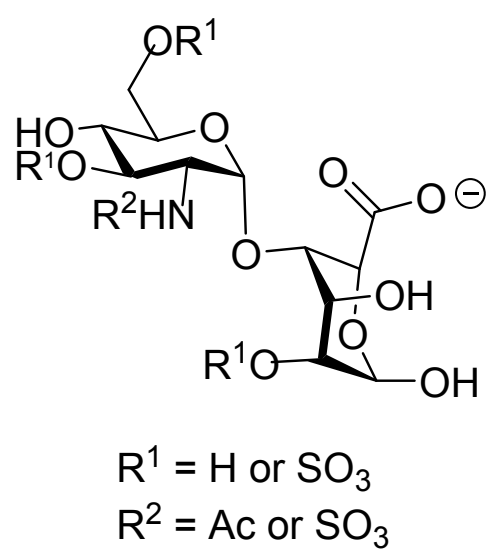


1. GlcNAc- $\alpha(1 \rightarrow 4)-\alpha-L-I d o A$

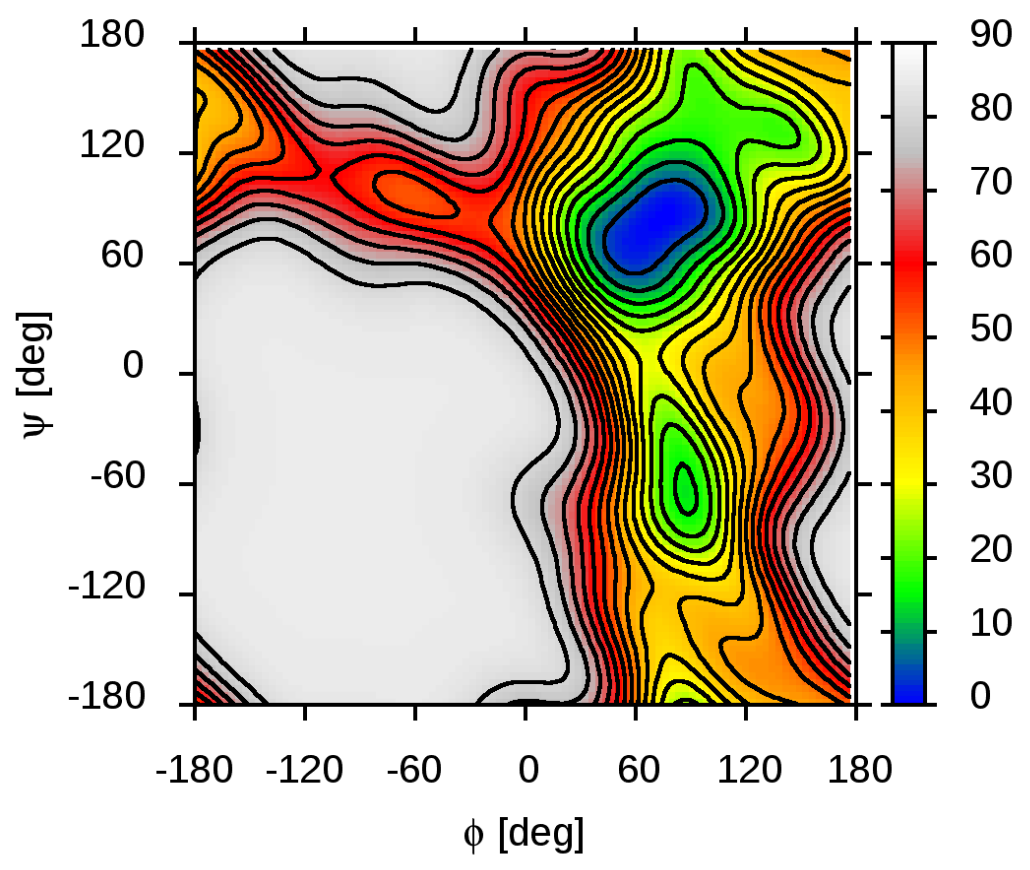

2. GlcNAc- $\alpha(1 \rightarrow 4)-\alpha-L-I d o A 2 S$

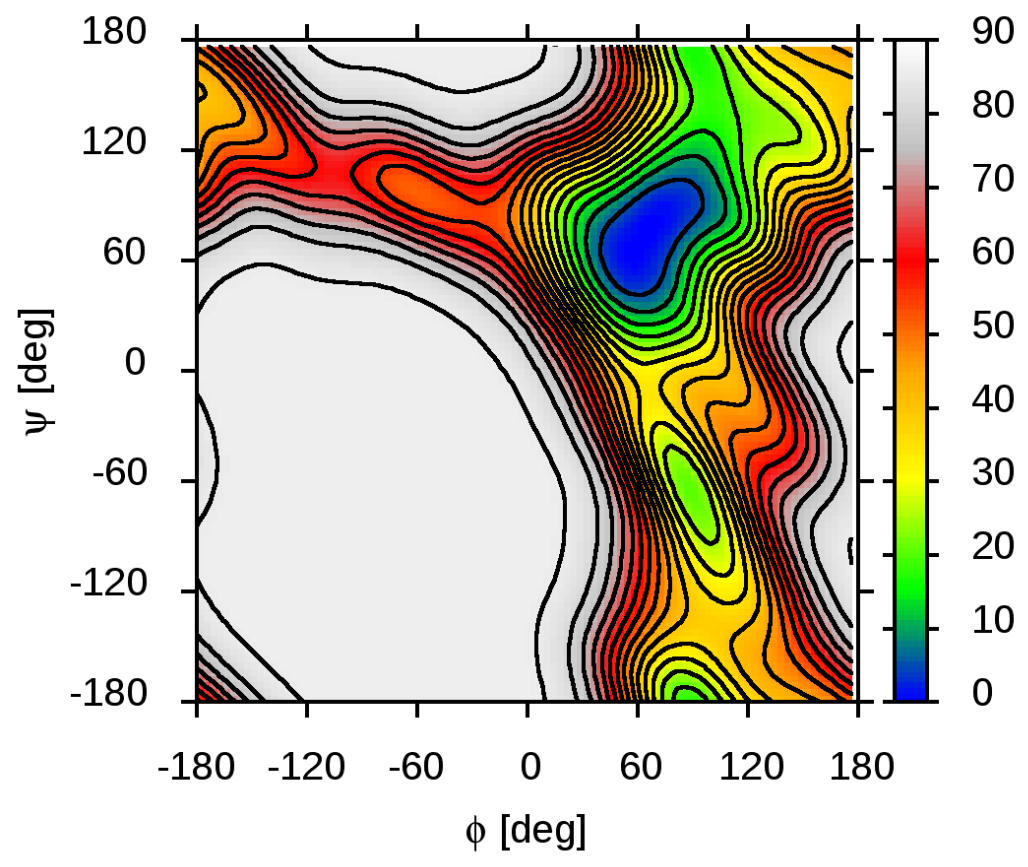


3. GlcNAc3S- $\alpha(1 \rightarrow 4)-\alpha-L-I d o A$

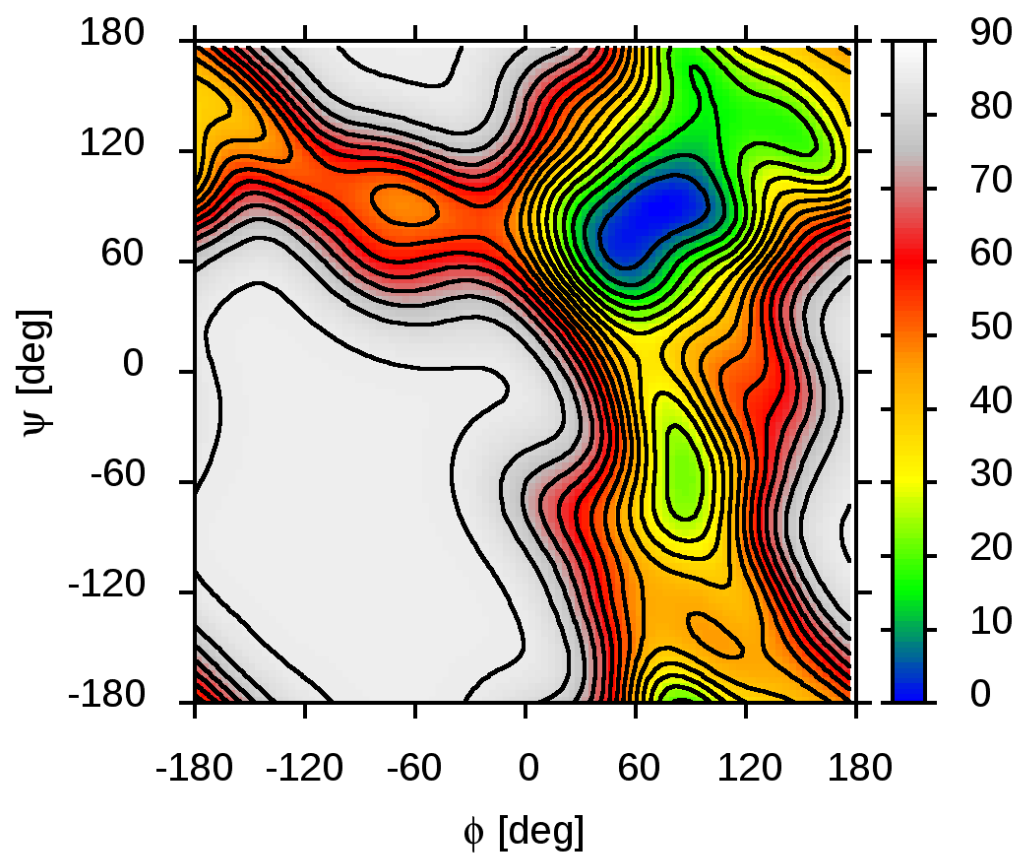

4. GlcNAc6S- $\alpha(1 \rightarrow 4)-\alpha-L-I d o A$

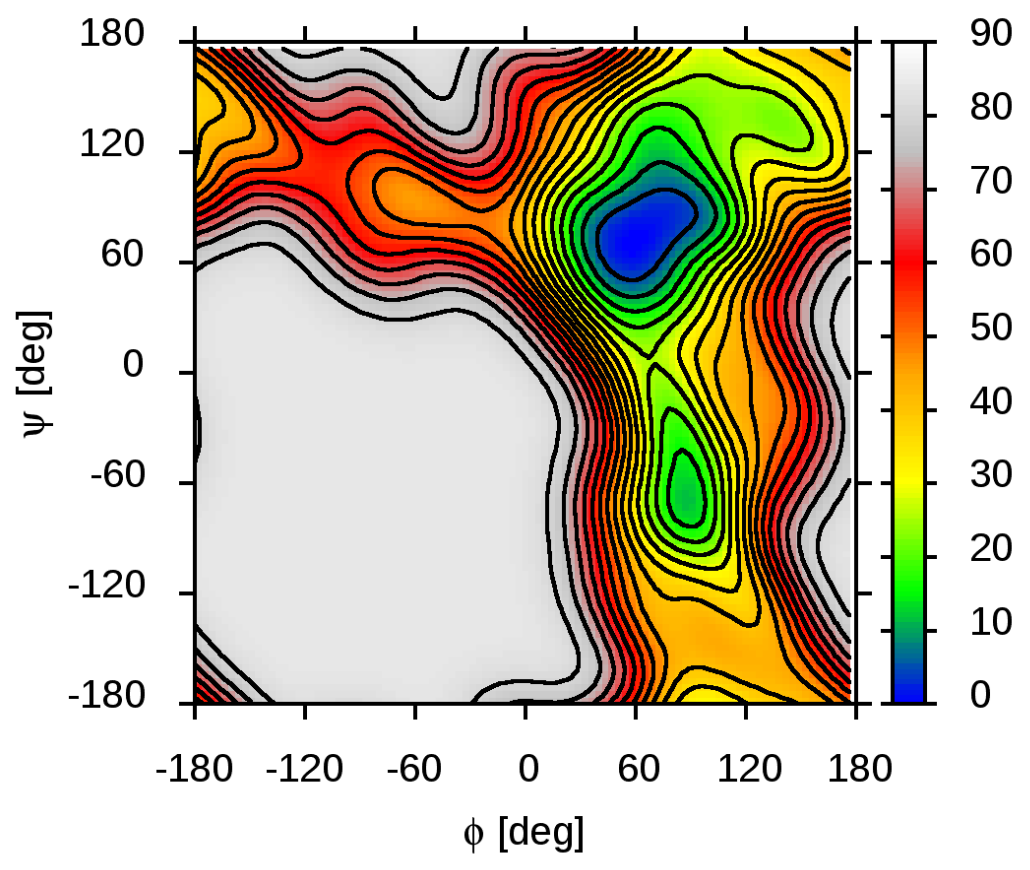


5. GlcNAc3S- $\alpha(1 \rightarrow 4)-\alpha-L-I d o A 2 S$

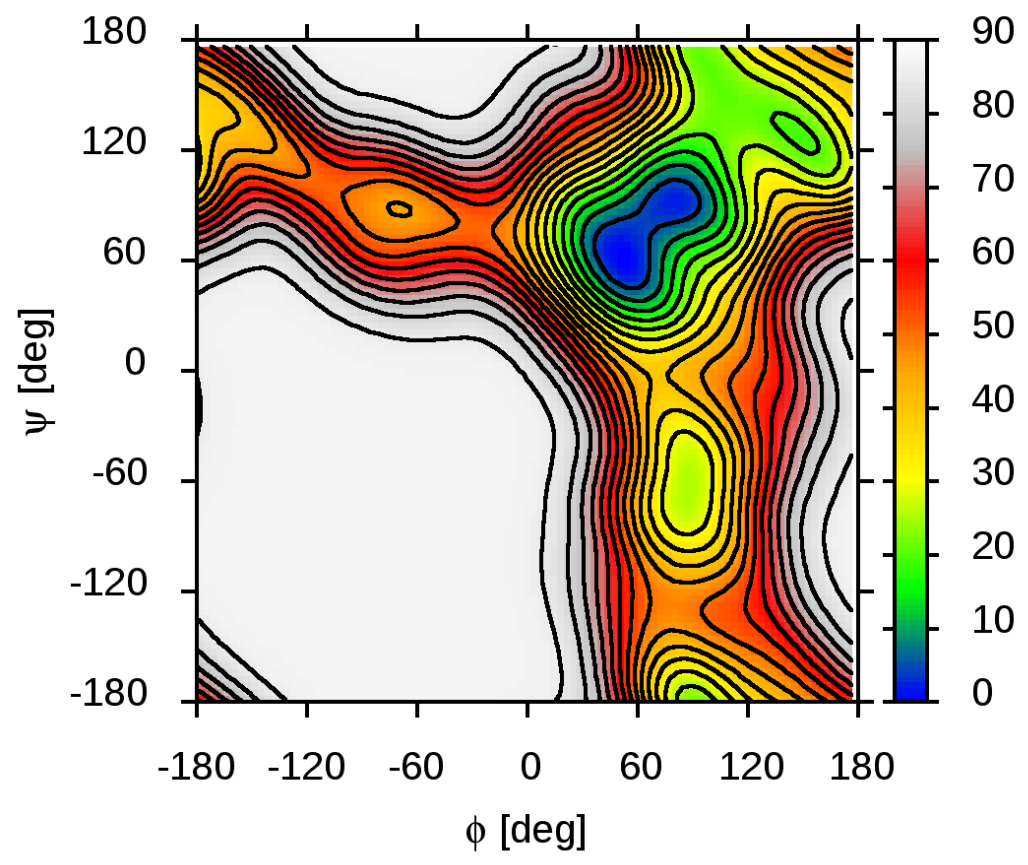

6. GlcNAc6S- $\alpha(1 \rightarrow 4)-\alpha-L-I d o A 2 S$

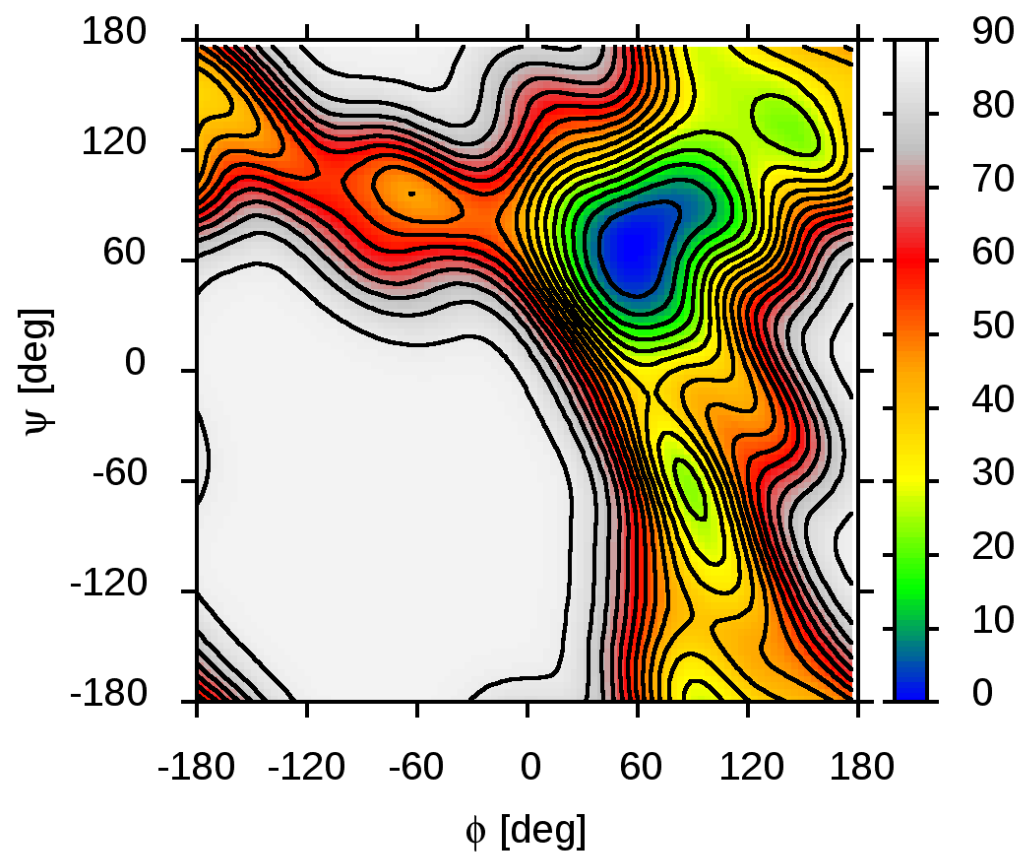


7. GlcNAc3,6S- $\alpha(1 \rightarrow 4)-\alpha-L-I d o A 2 S$

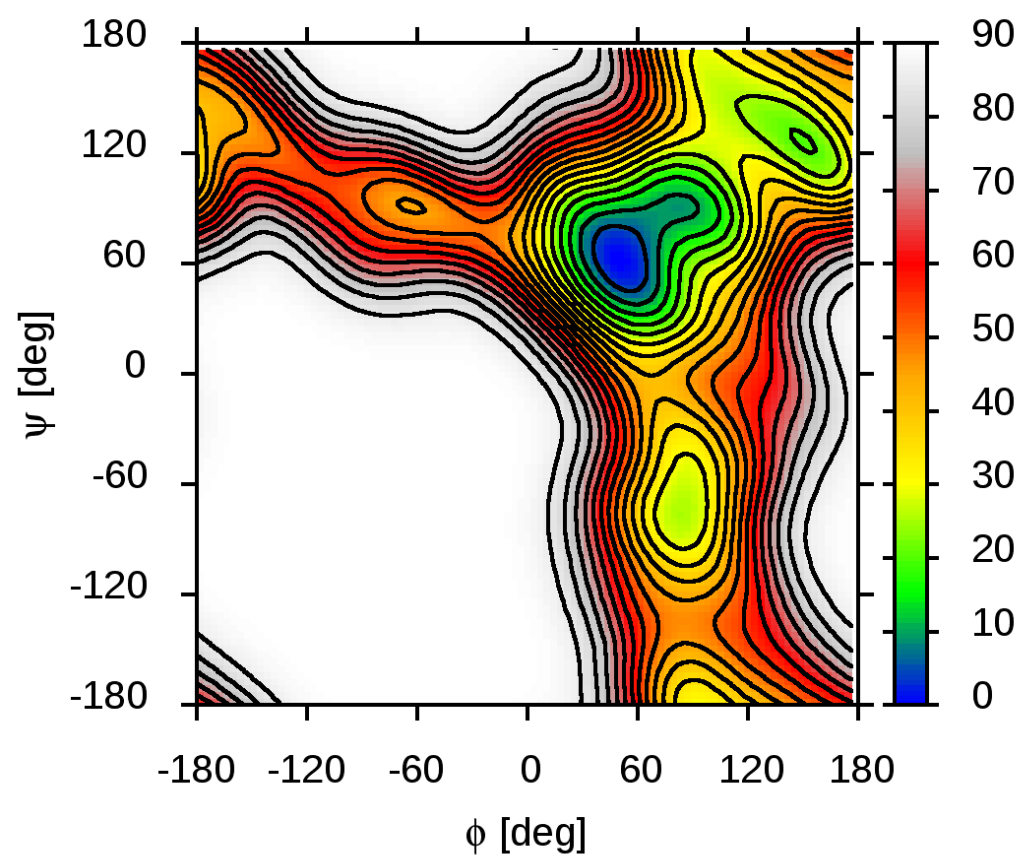

8. GlcNAc3,6S- $\alpha(1 \rightarrow 4)-\alpha-L-I d o A$

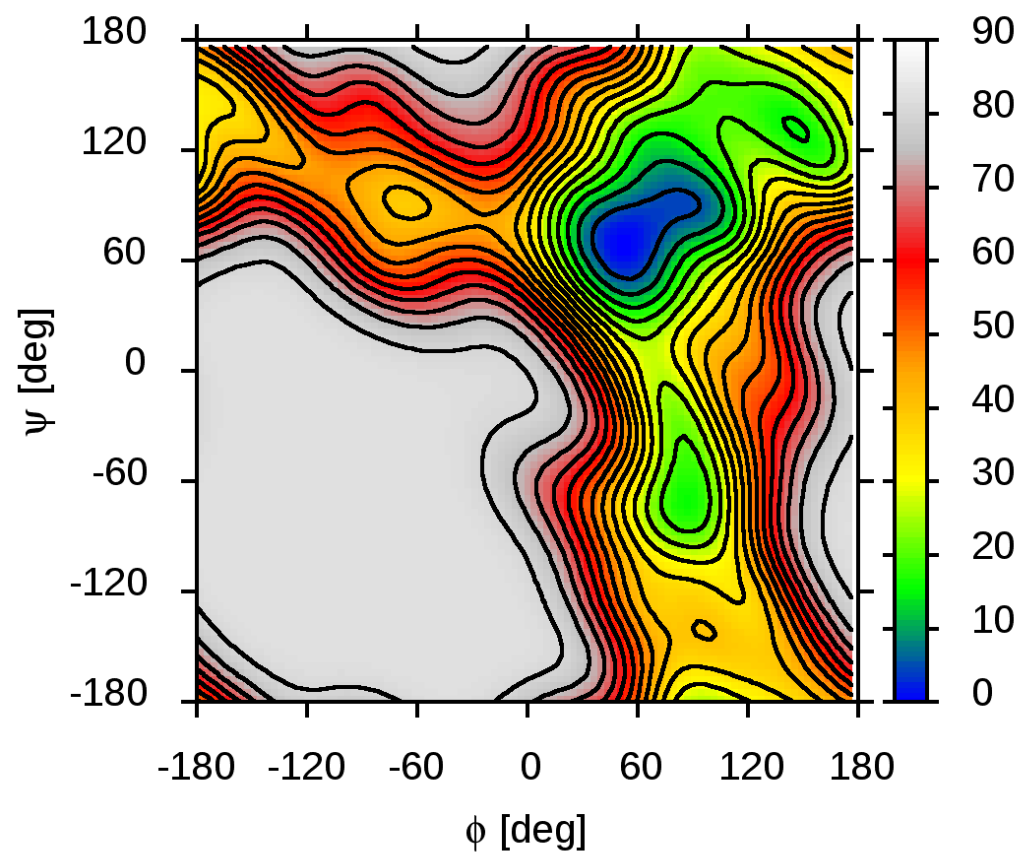


9. GlcNS- $\alpha(1 \rightarrow 4)-\alpha-L-I d o A$

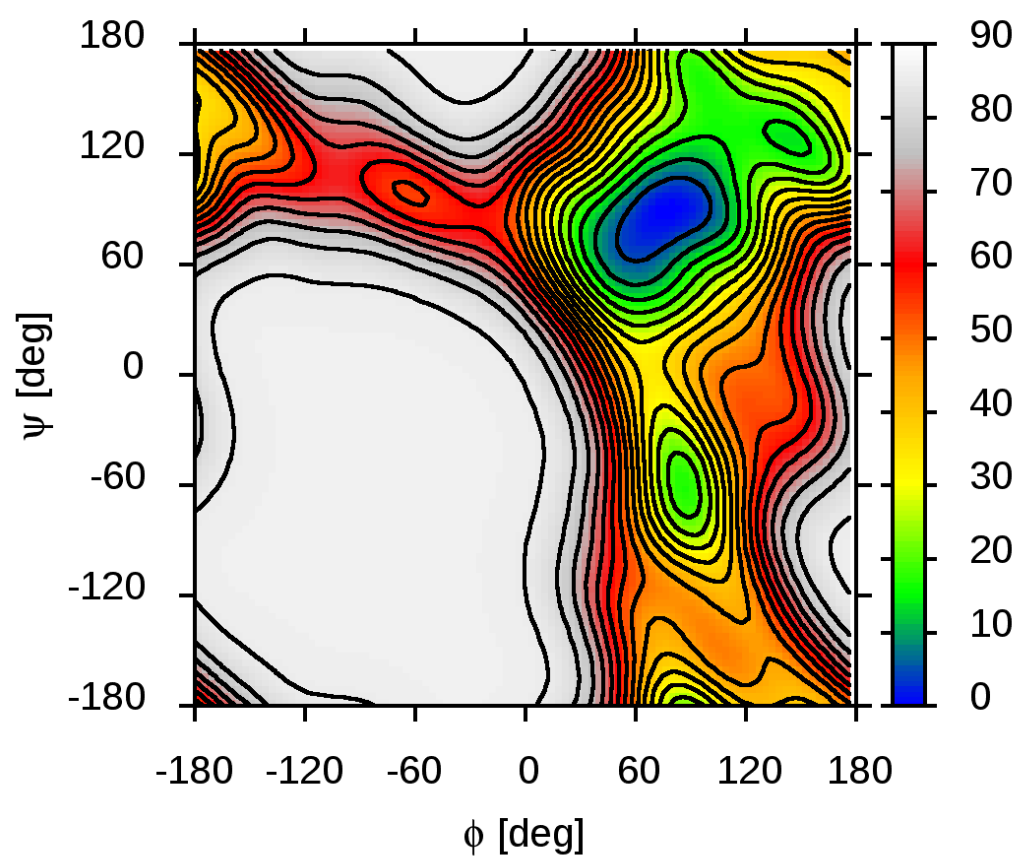

10. GlcNS- $\alpha(1 \rightarrow 4)-\alpha-L-I d o A 2 S$

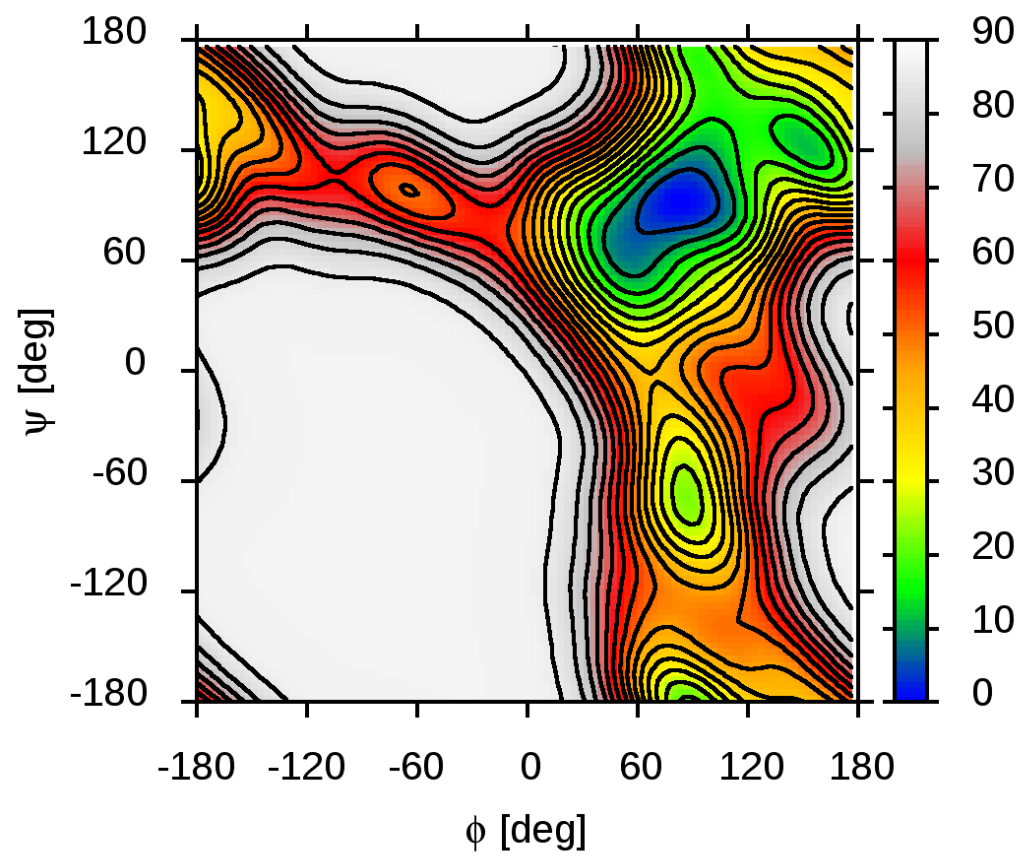


11. GlcNS3S- $\alpha(1 \rightarrow 4)-\alpha-$-LIdoA

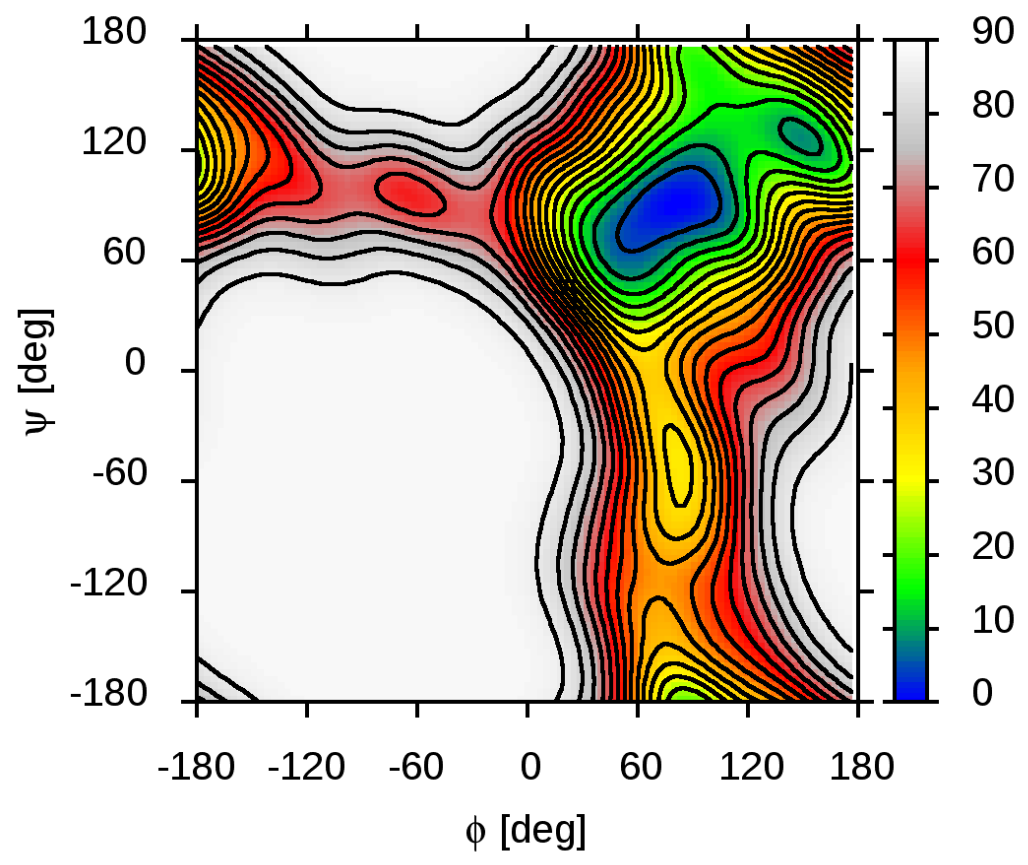

12. GlcNS6S- $\alpha(1 \rightarrow 4)-\alpha-$-LIdoA

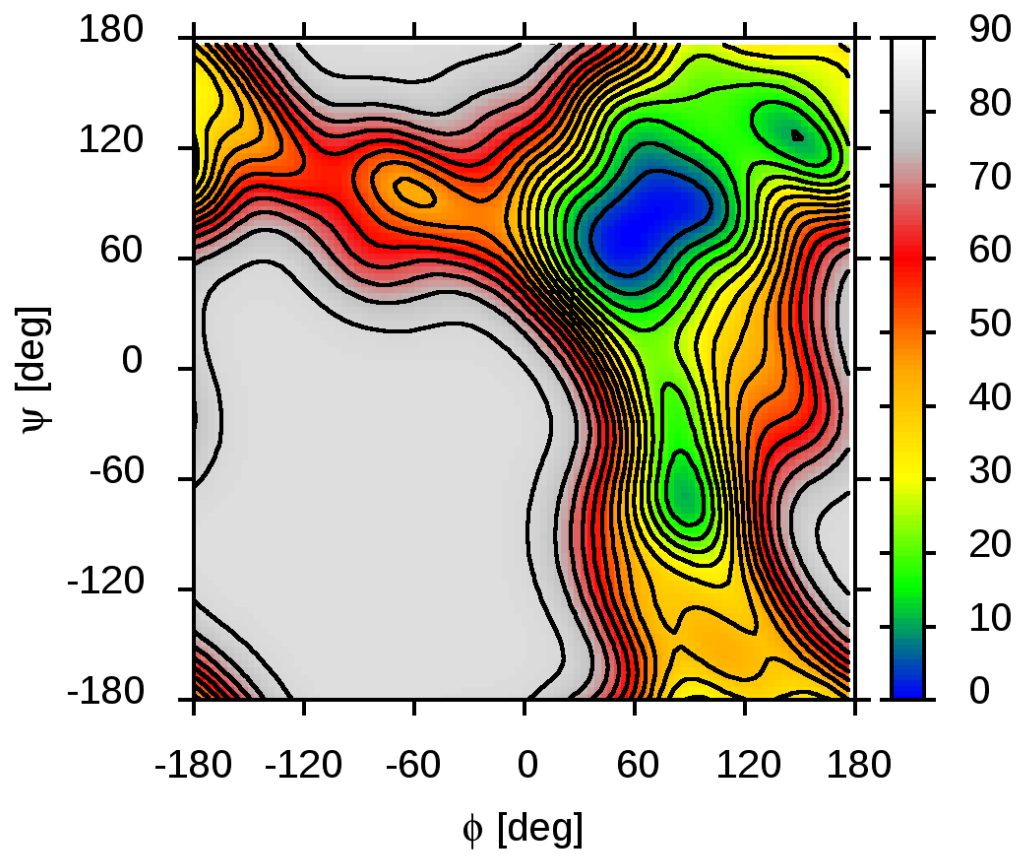


13. GlcNS3S- $\alpha(1 \rightarrow 4)-\alpha-$-LIdoA2S

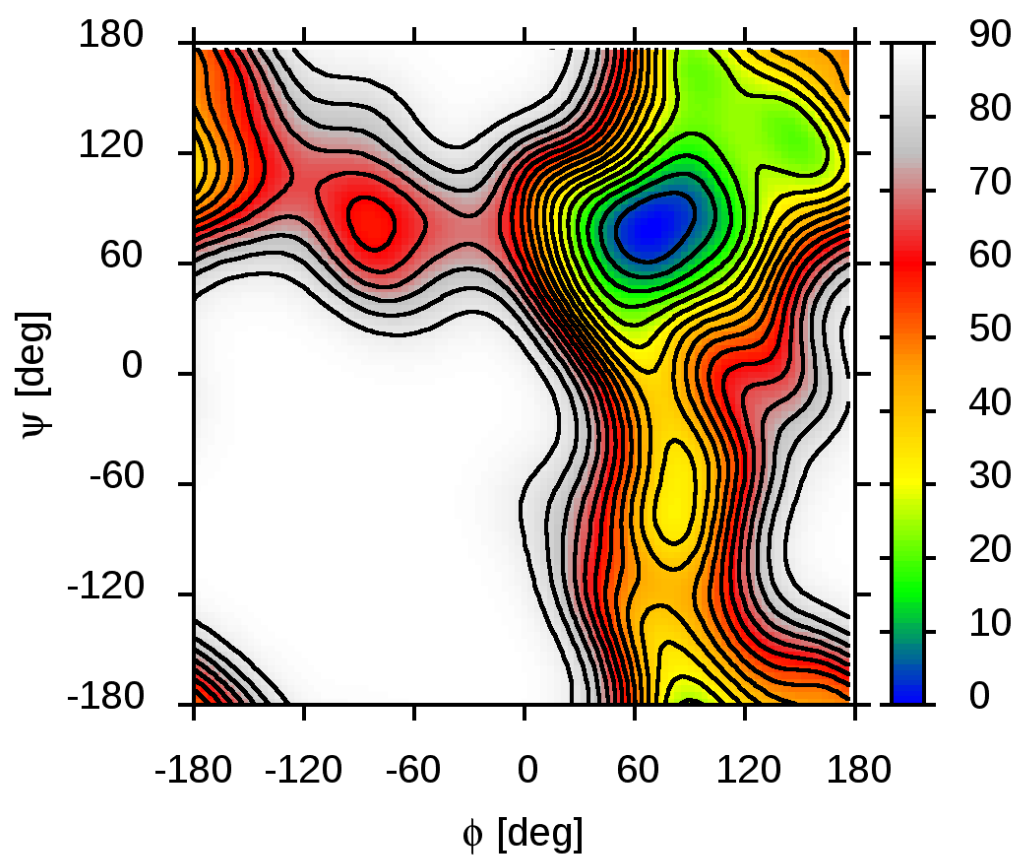

14. GlcNS6S- $\alpha(1 \rightarrow 4)-\alpha-$-L-IdoA2S

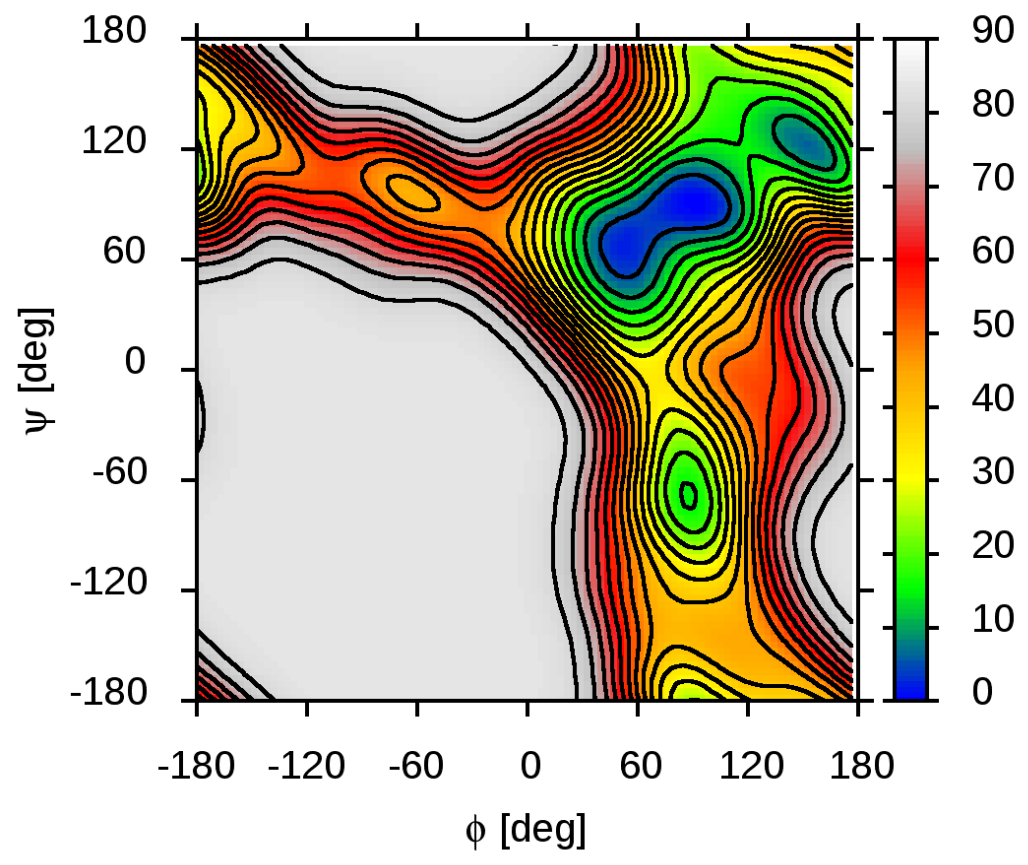


15. GlcNS3,6S-a(1 $\rightarrow 4)-\alpha-L-I d o A$

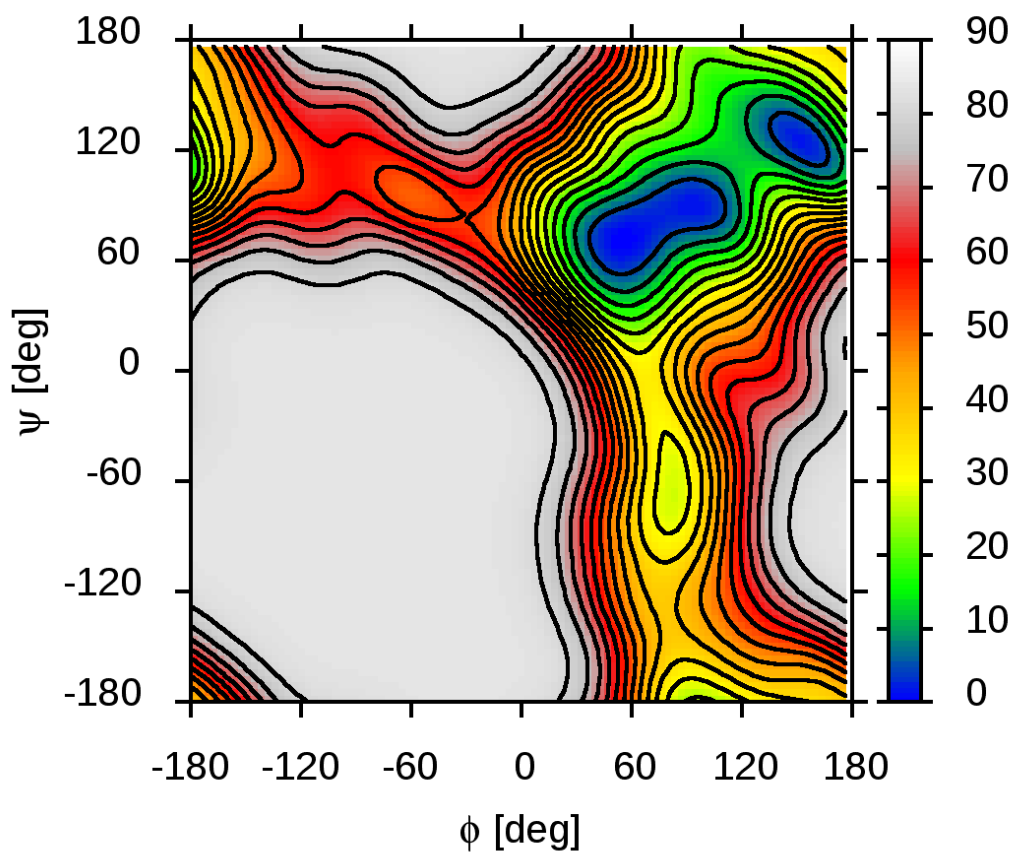

16. GlcNS3,6S- $\alpha(1 \rightarrow 4)-\alpha-L-I d o A 2 S$

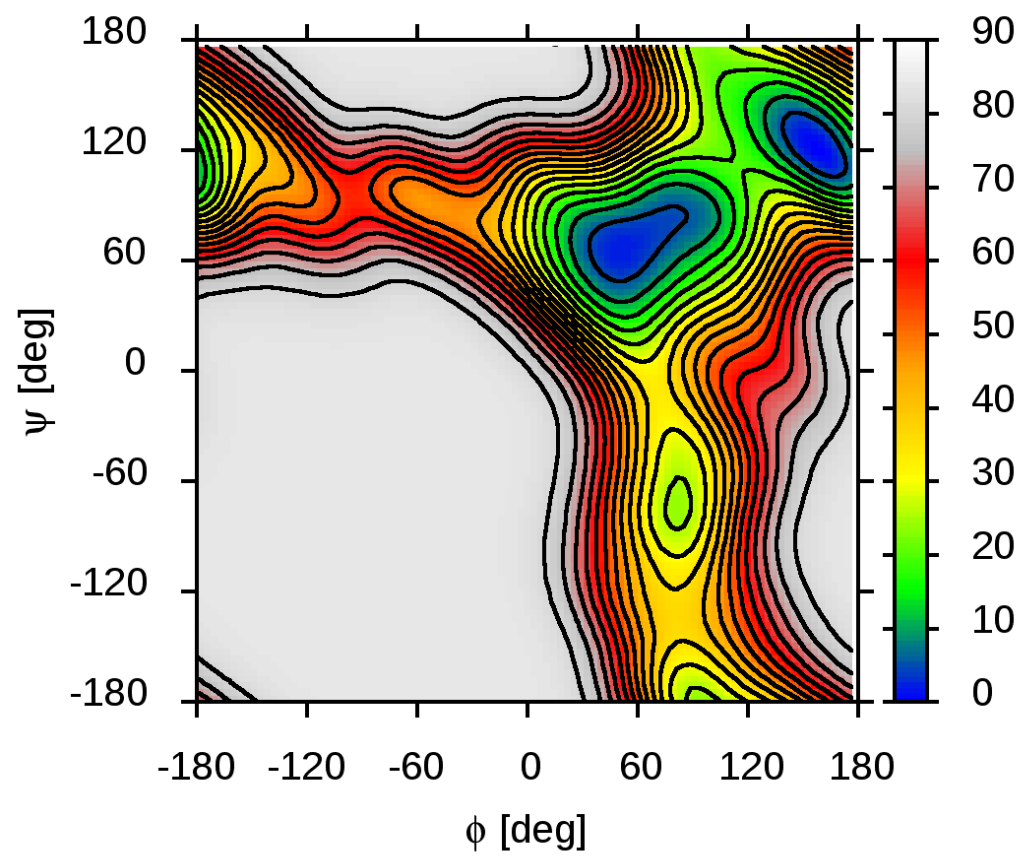


c. $G \mid c A 2 R^{1}-\beta(1 \rightarrow 4)-\alpha-G_{c} N R^{2} 3,6 R^{1}$

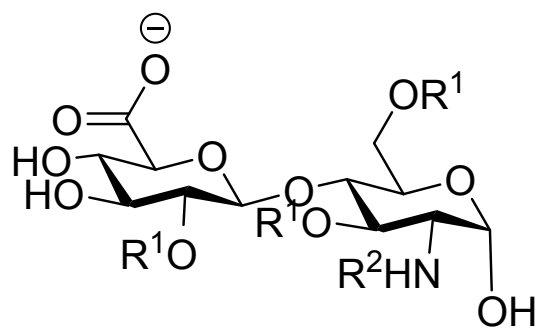

$$
\begin{aligned}
& \mathrm{R}^{1}=\mathrm{H} \text { or } \mathrm{SO}_{3} \\
& \mathrm{R}^{2}=\mathrm{Ac} \text { or } \mathrm{SO}_{3}
\end{aligned}
$$

1. GlcA- $\beta(1 \rightarrow 4)-\alpha-G \mid c N A c$

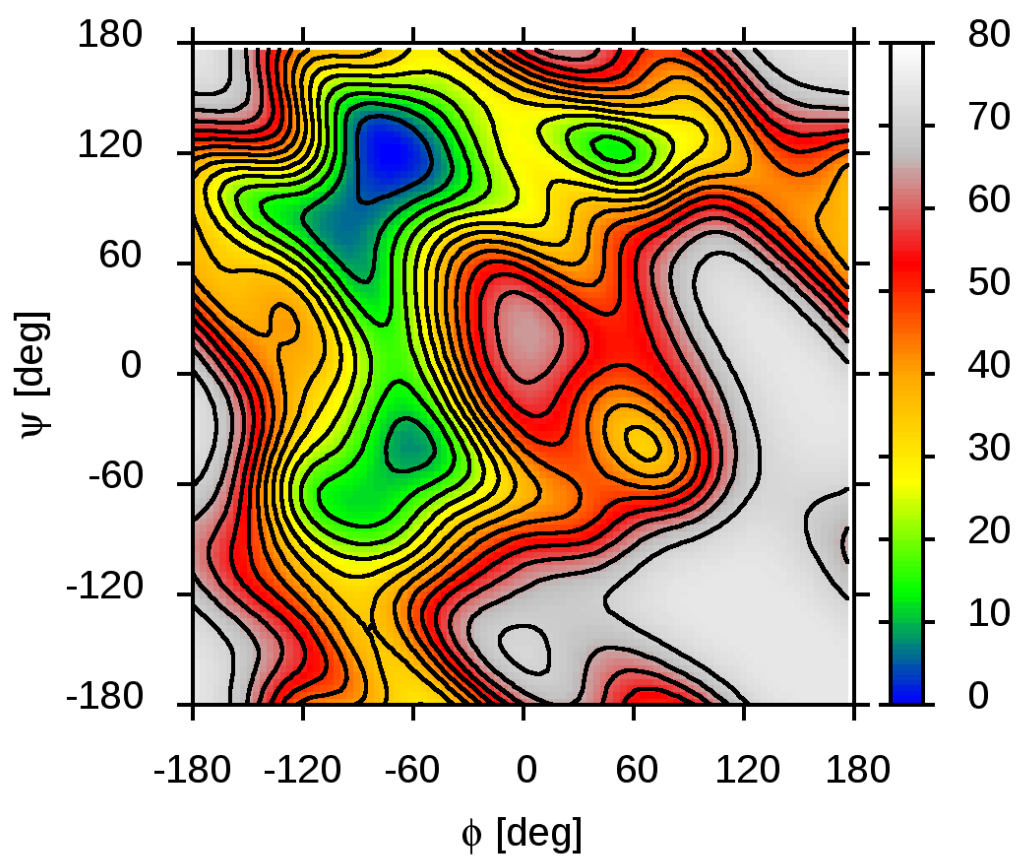


2. GlcA- $\beta(1 \rightarrow 4)-\alpha-G l c N A c 3 S$

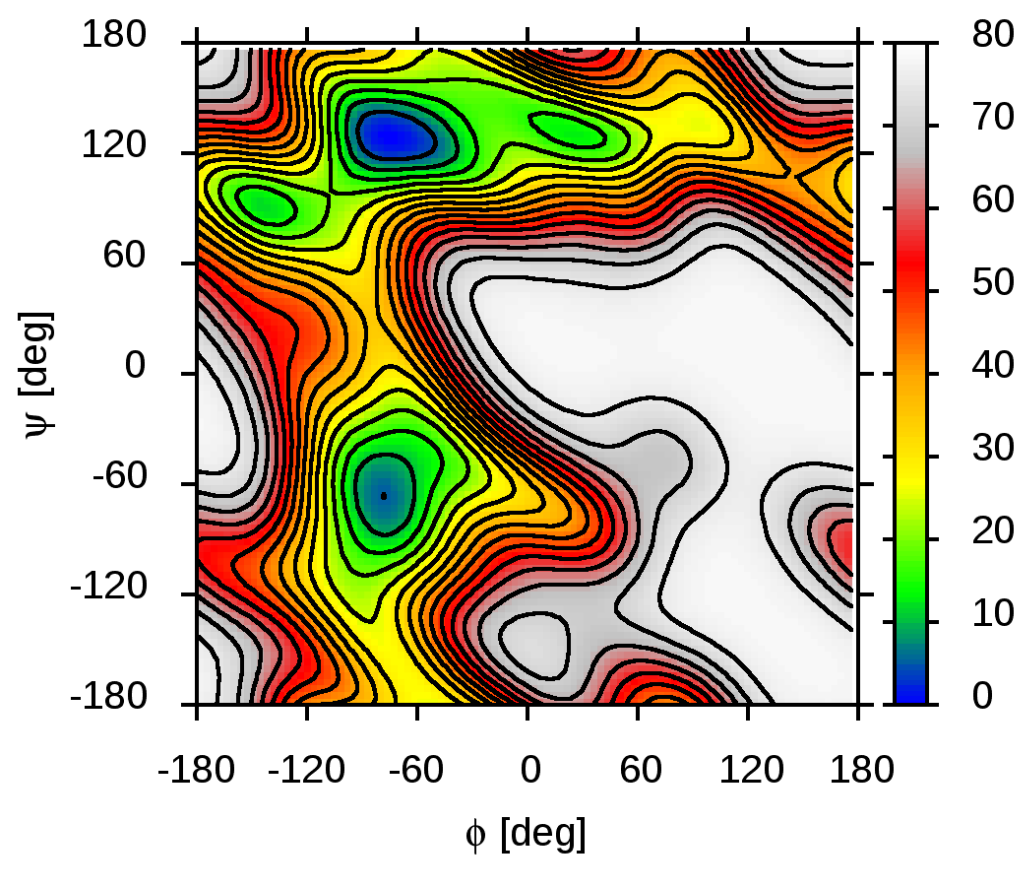

3. GlcA- $\beta(1 \rightarrow 4)-\alpha-G l c N A c 6 S$

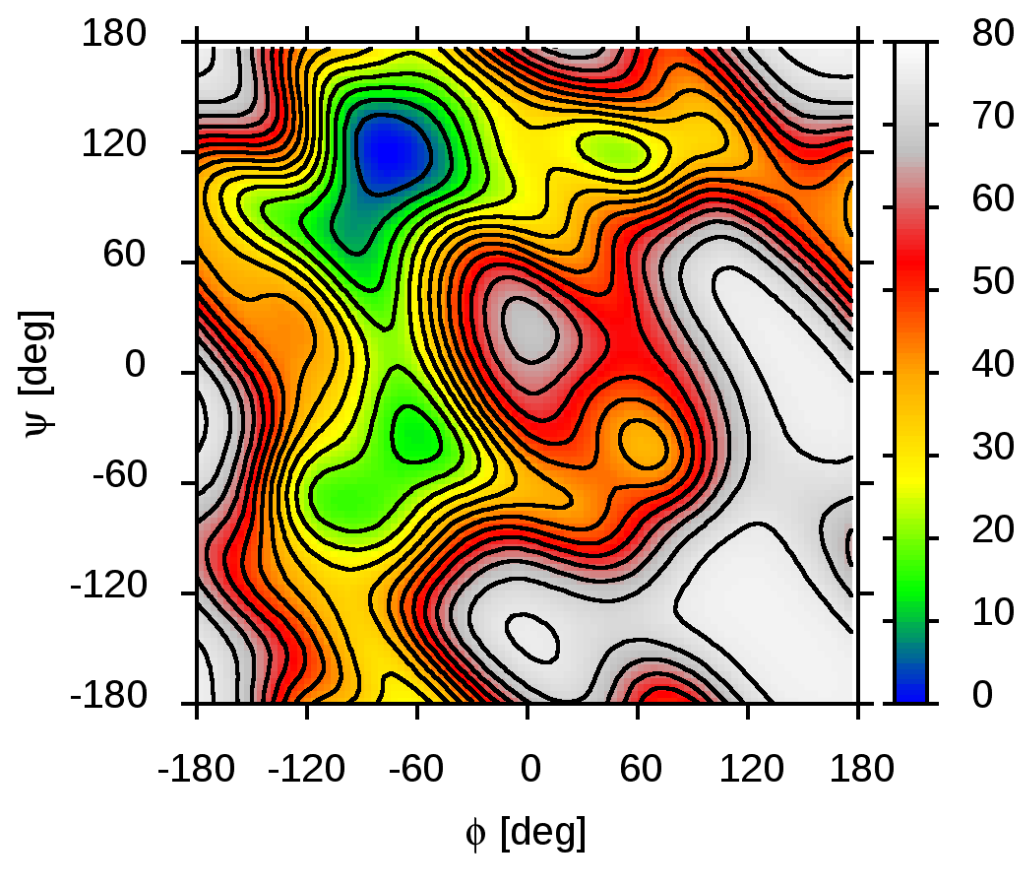


4. GlcA2S- $\beta(1 \rightarrow 4)-\alpha-G l c N A c$

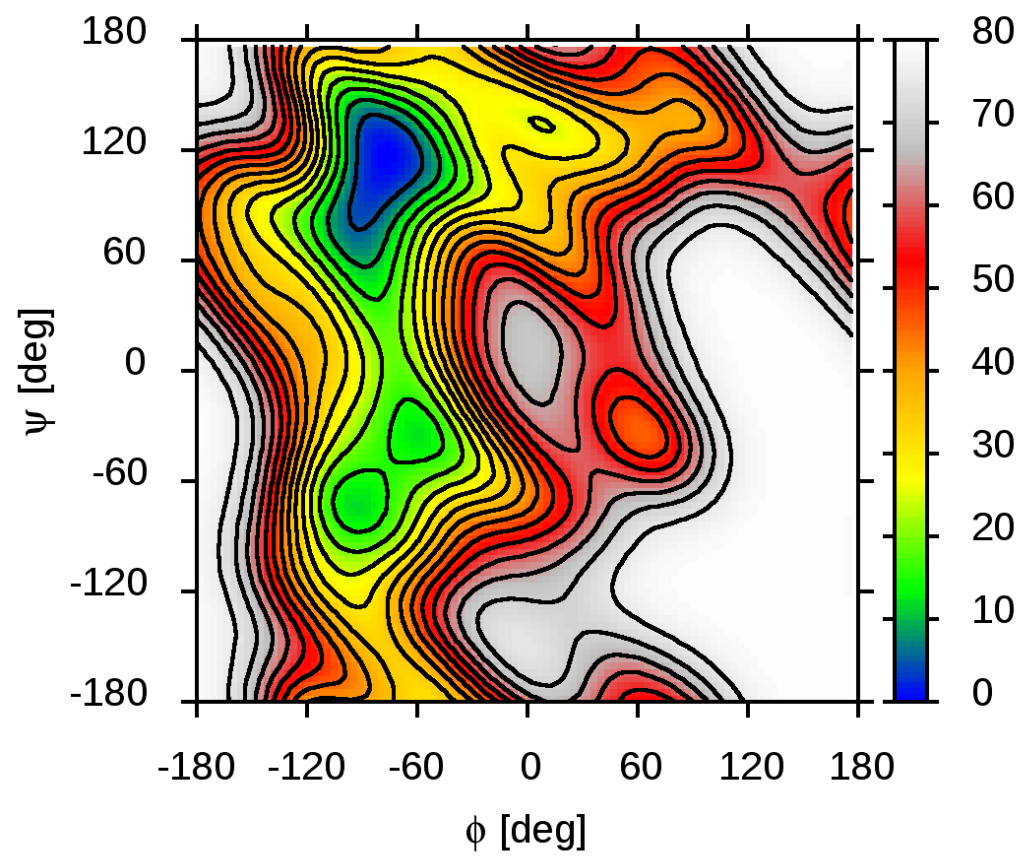

5. GlcA2S- $\beta(1 \rightarrow 4)-\alpha-$ GlcNAc3S

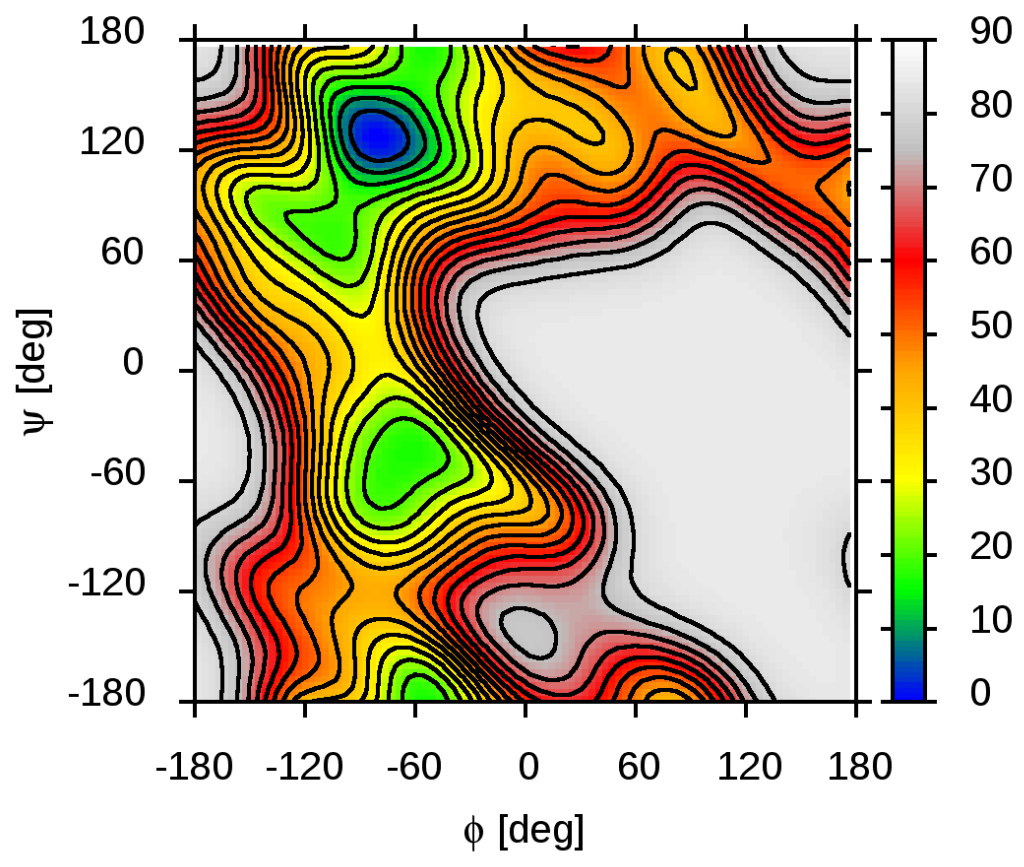


6. GlcA2S- $\beta(1 \rightarrow 4)-\alpha-G l c N A c 6 S$

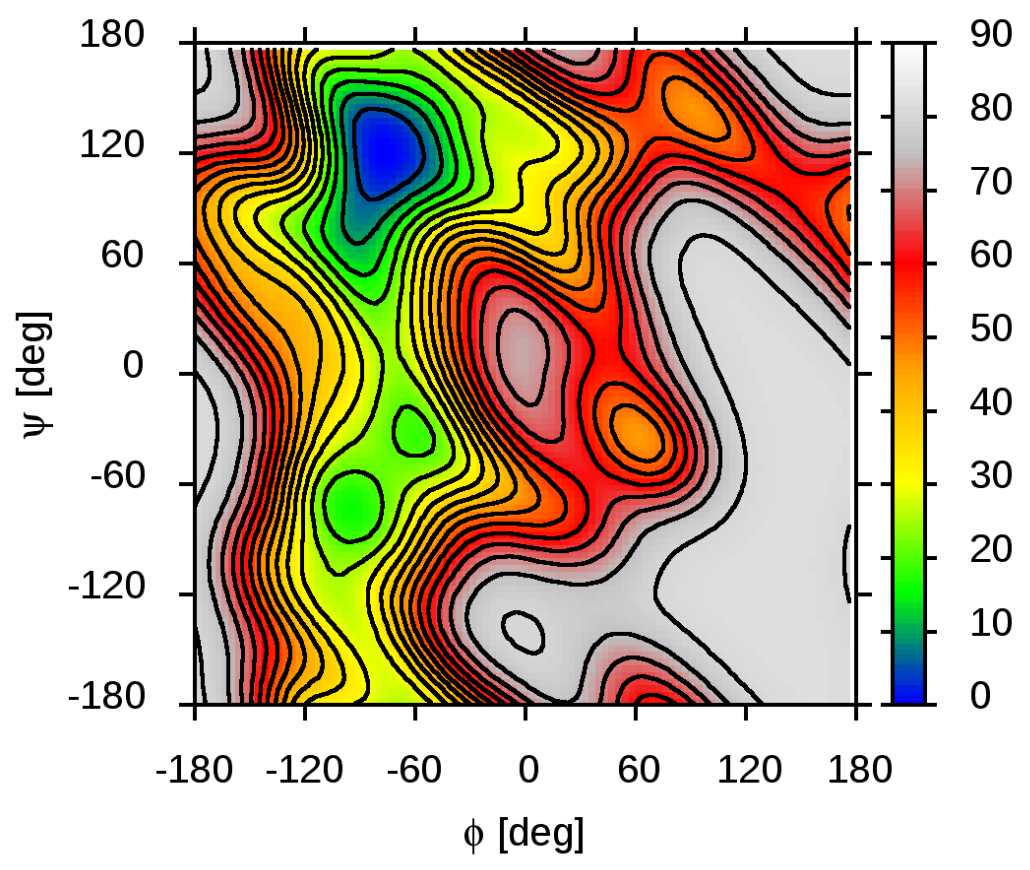

7. $\operatorname{GlcA}-\beta(1 \rightarrow 4)-\alpha-G \mid c N A c 3,6 S$

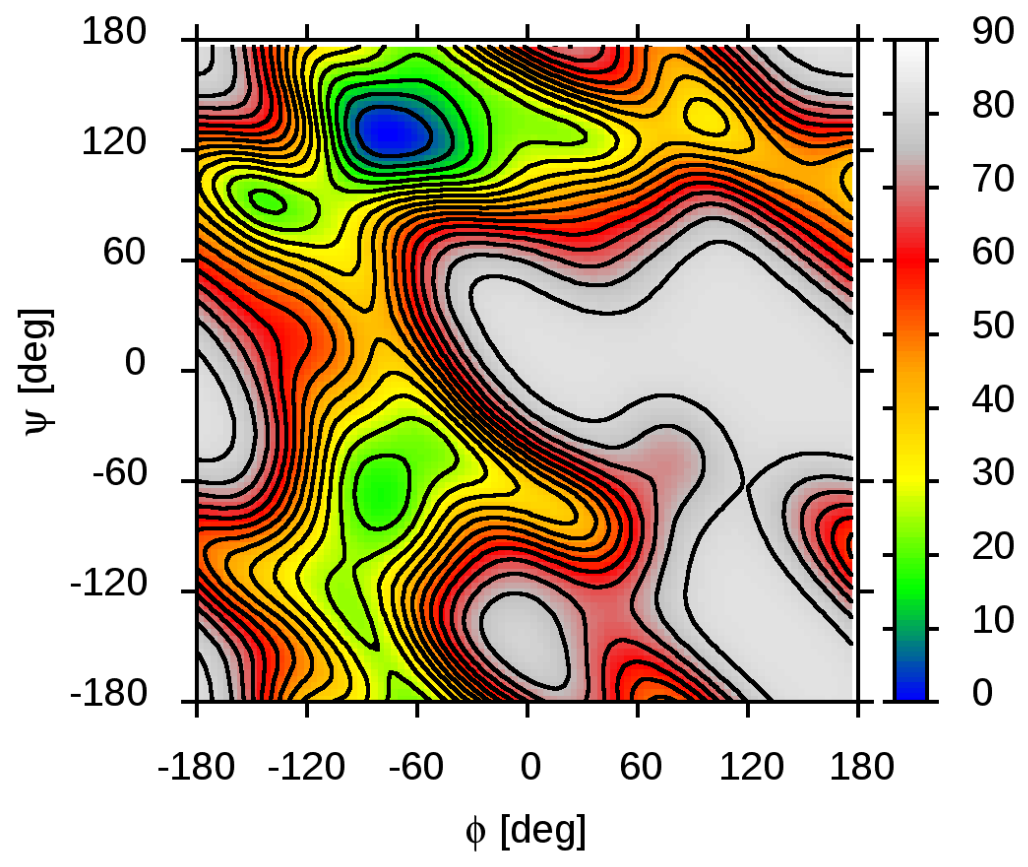


8. GlcA2S- $\beta(1 \rightarrow 4)-\alpha-$ GlcNAc3,6S

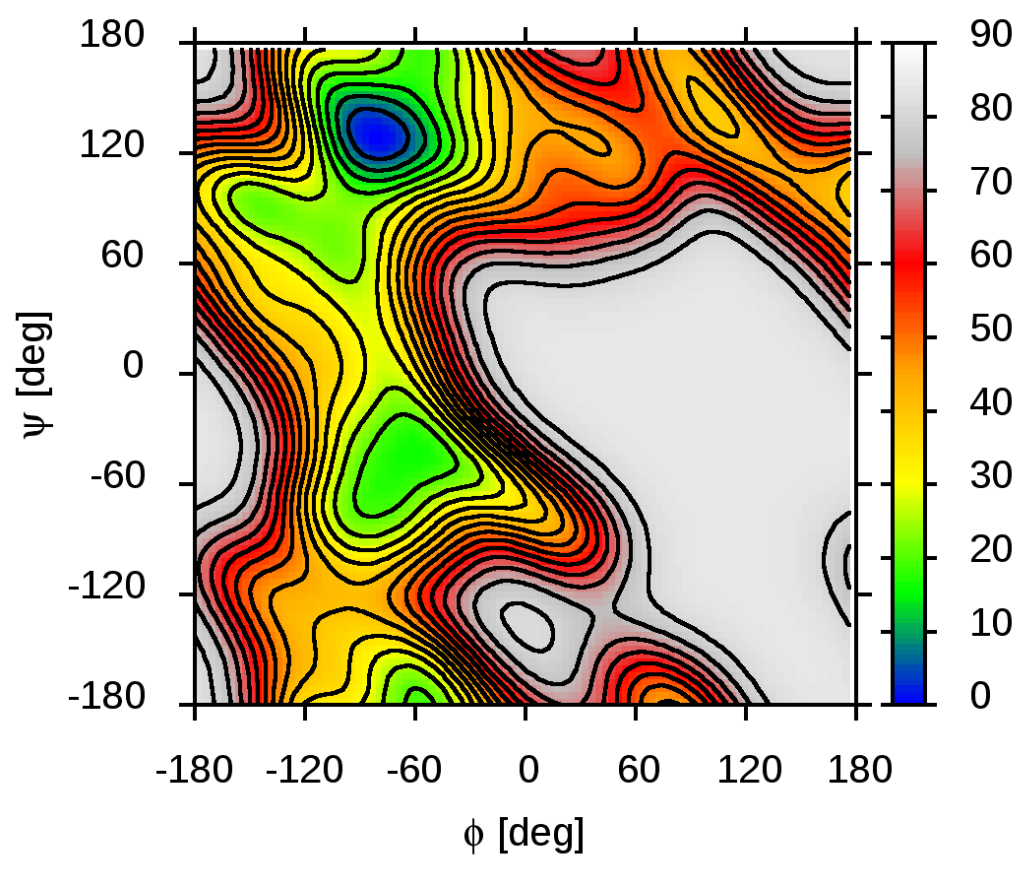

9. GlcA- $\beta(1 \rightarrow 4)-\alpha-G \mid c N S$

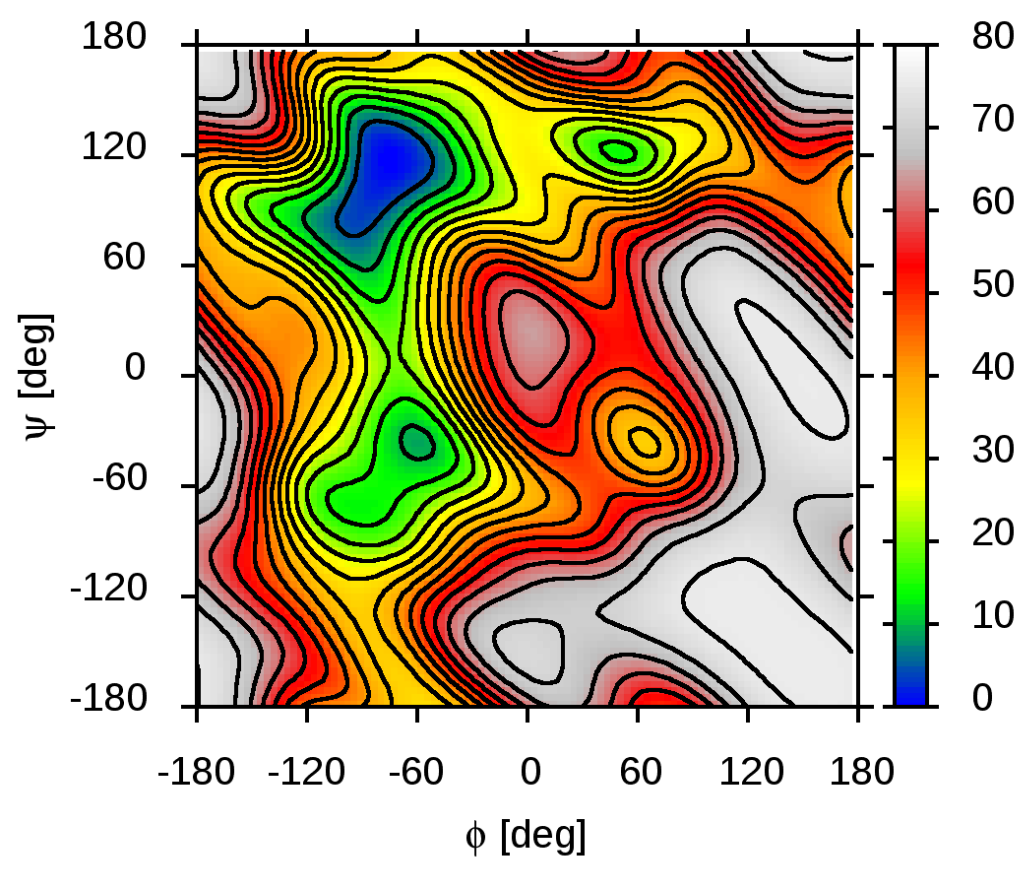


10. GlcA- $\beta(1 \rightarrow 4)-\alpha-$ GlcNS3S

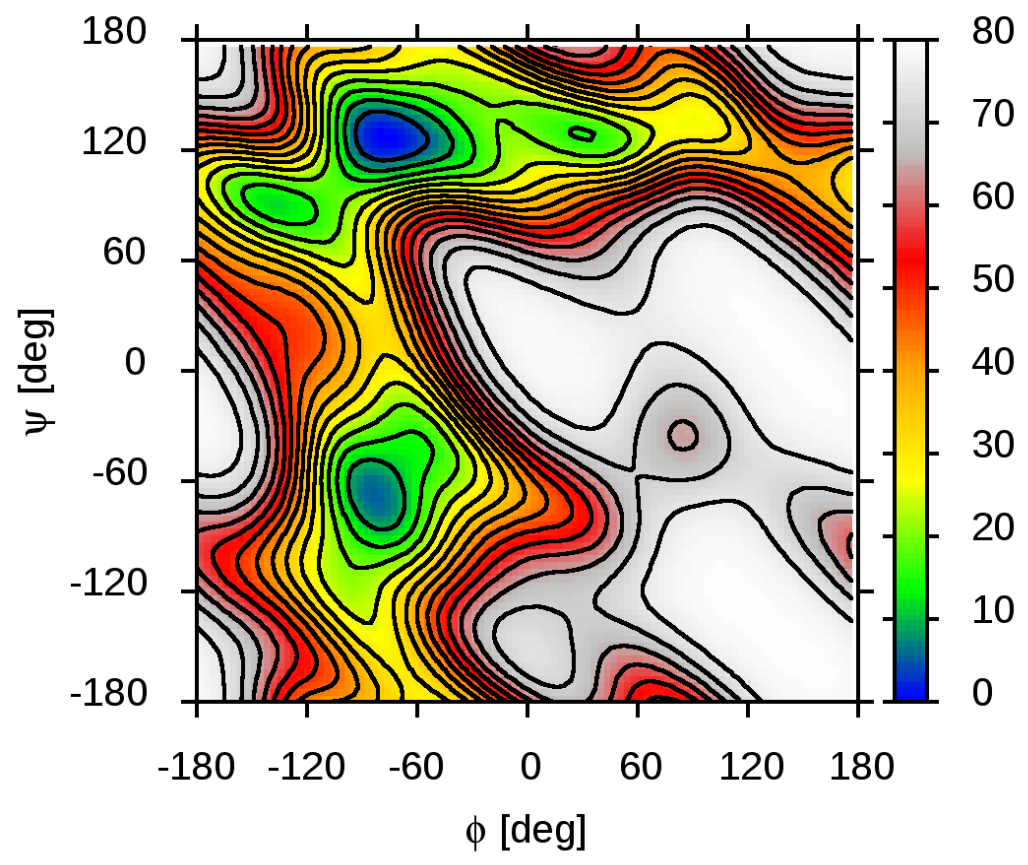

11. GlcA- $\beta(1 \rightarrow 4)-\alpha-$ GlcNS6S

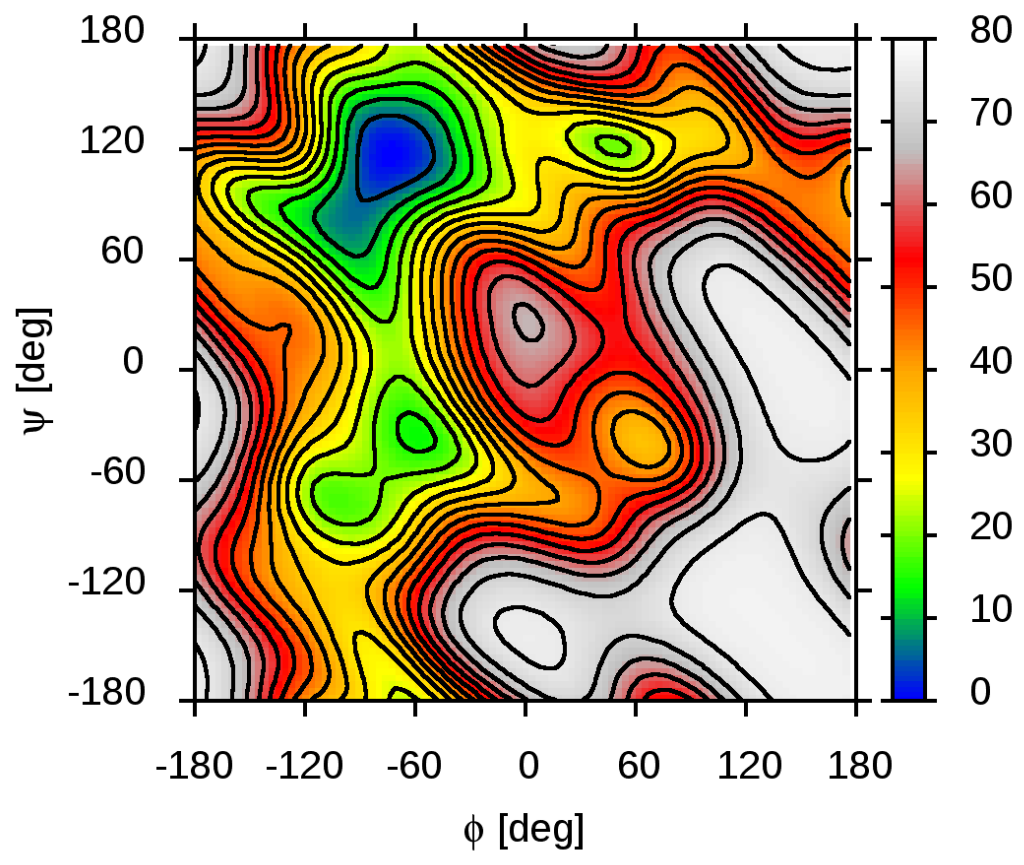


12. GlcA2S- $\beta(1 \rightarrow 4)-\alpha-G \mid c N S$

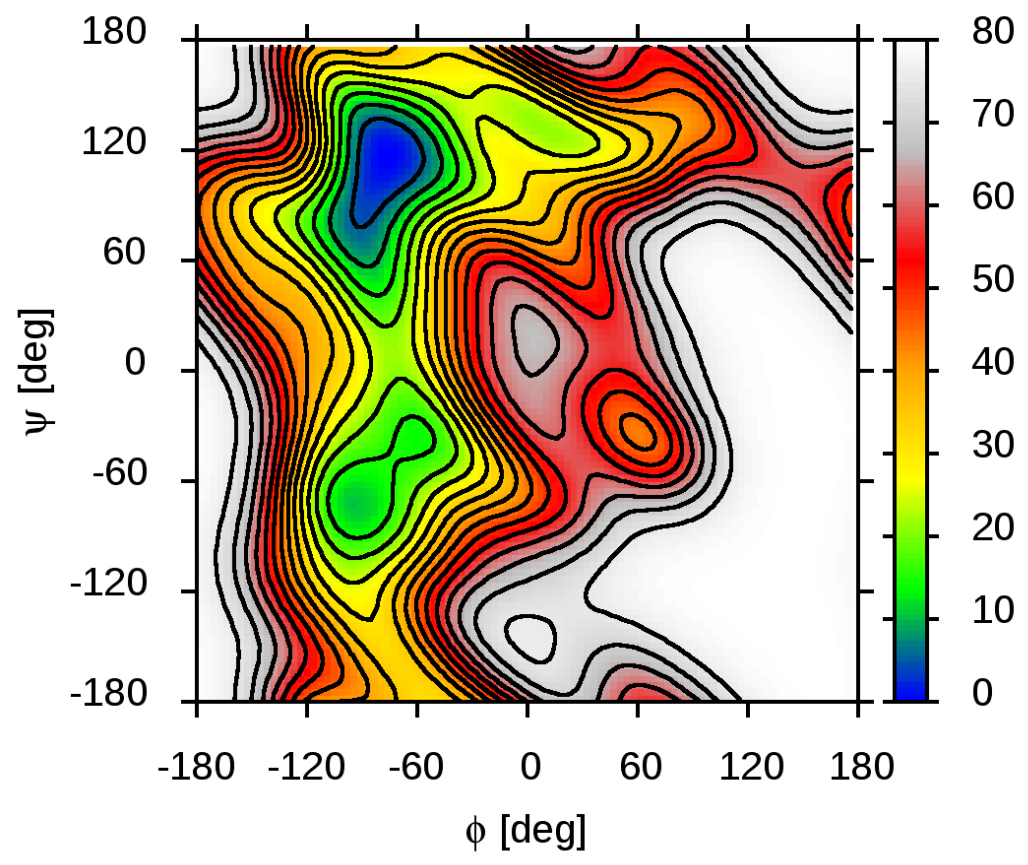

13. GlcA2S- $\beta(1 \rightarrow 4)-\alpha-G \mid c N S 3 S$

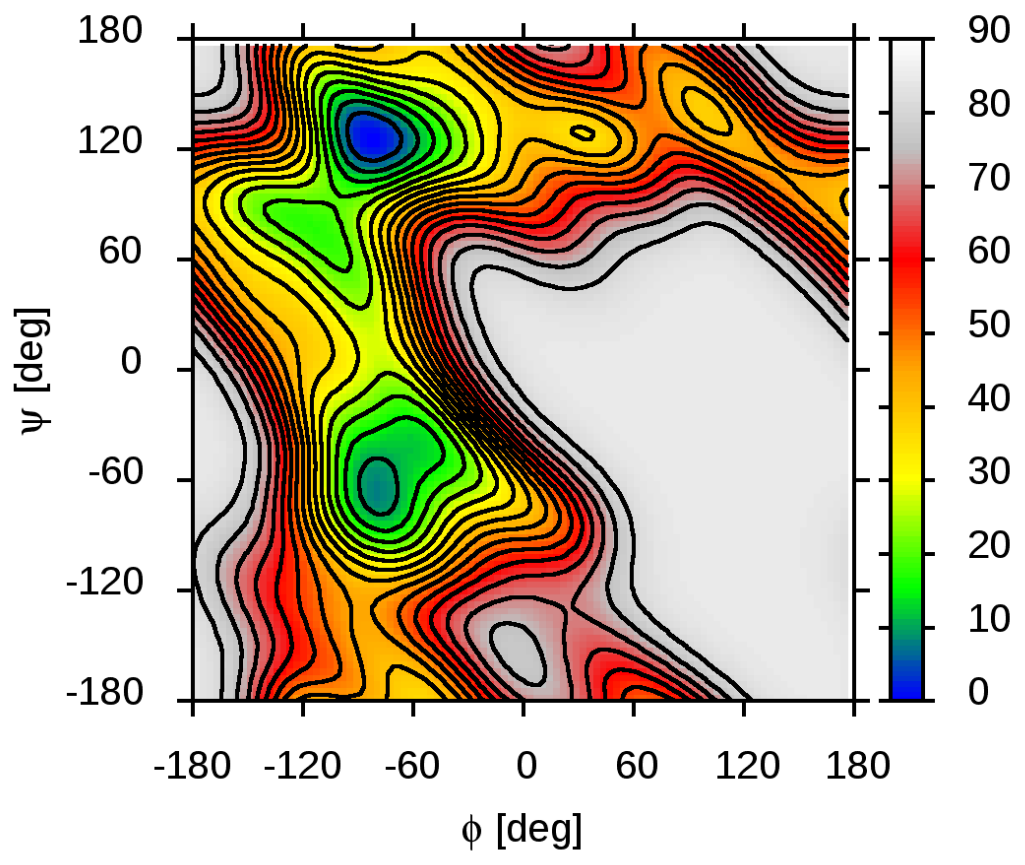


14. GlcA2S- $\beta(1 \rightarrow 4)-\alpha-$ GlcNS6S

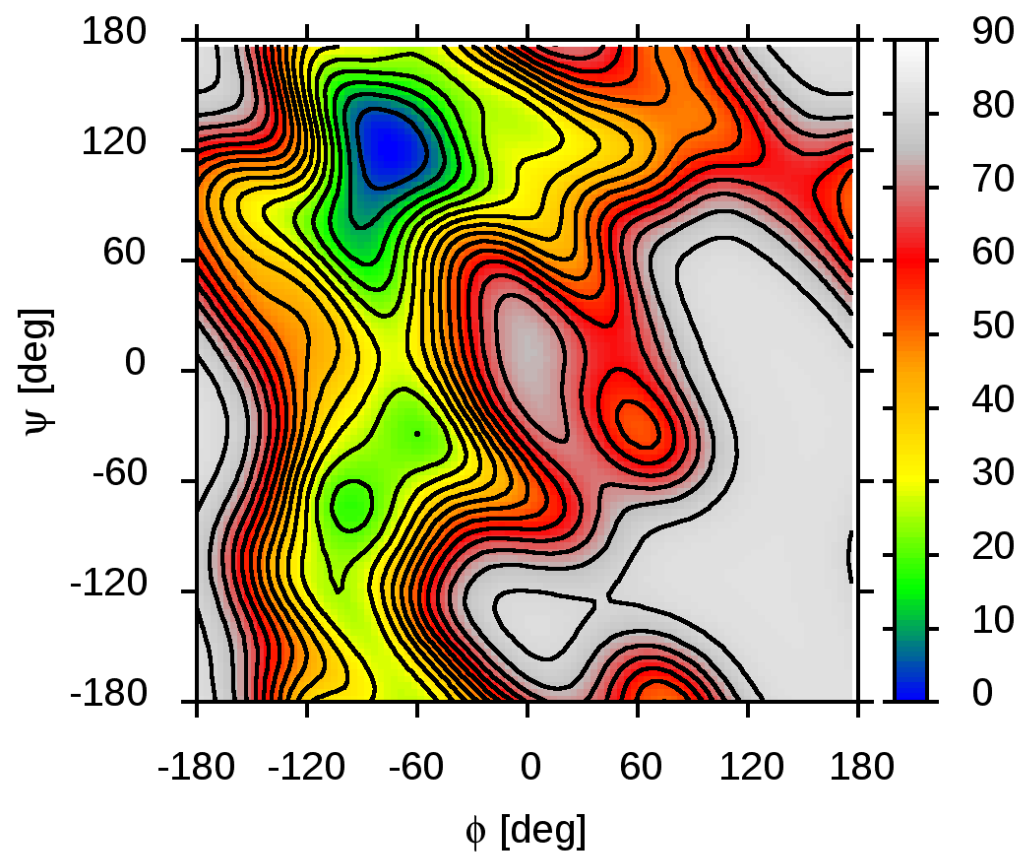

15. GlcA- $\beta(1 \rightarrow 4)-\alpha-G \mid c N S 3,6 S$

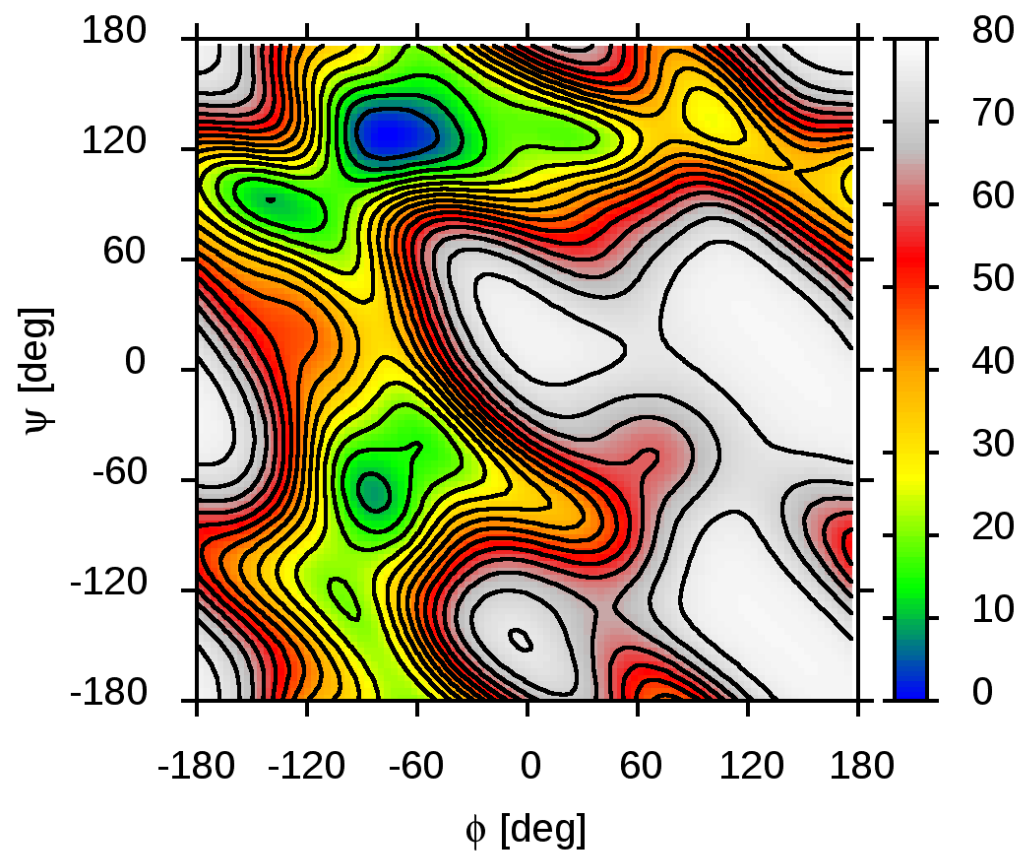


16. GlcA2S- $\beta(1 \rightarrow 4)-\alpha-G \mid c N S 3,6 S$

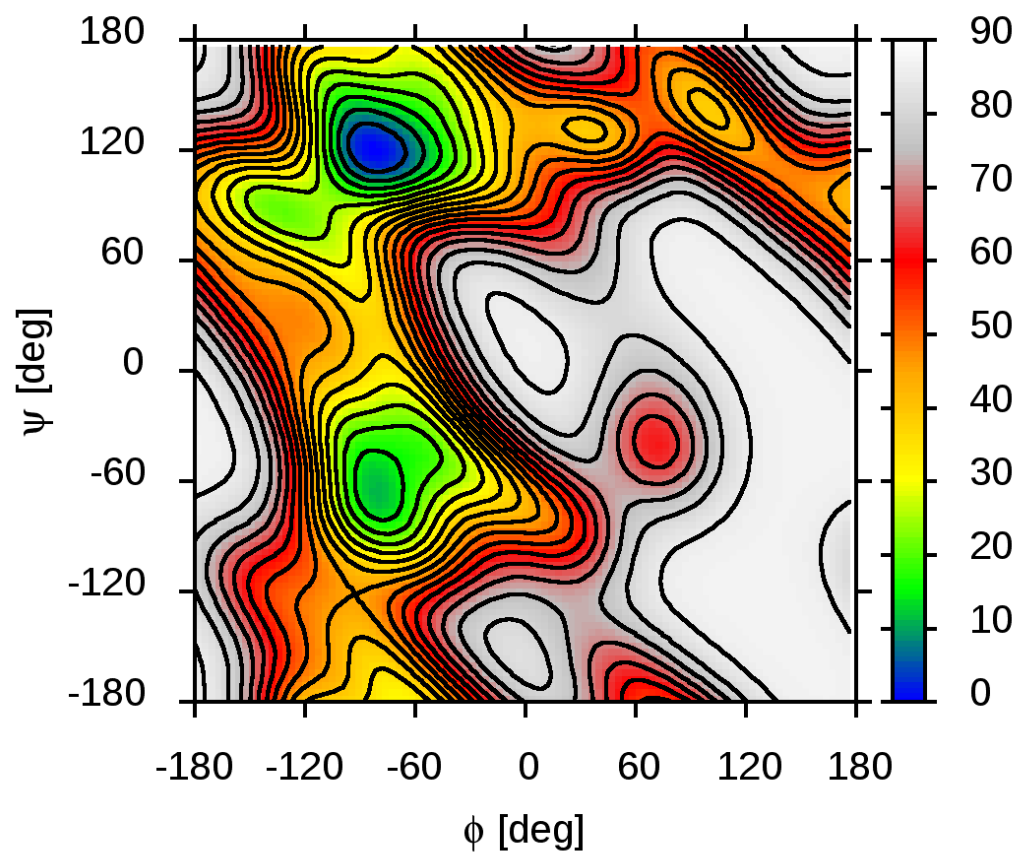

d. $G_{1 c N R}^{23}, 6 R^{1}-\alpha(1 \rightarrow 4)-\beta-G \mid c A 2 R^{1}$

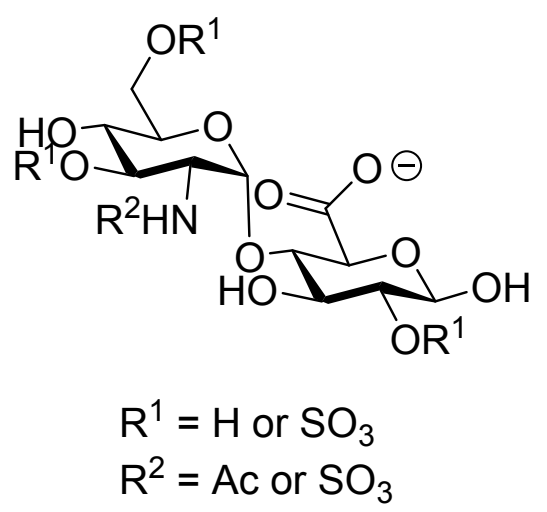


1. GlcNAc- $\alpha(1 \rightarrow 4)-\beta-G \mid c A$

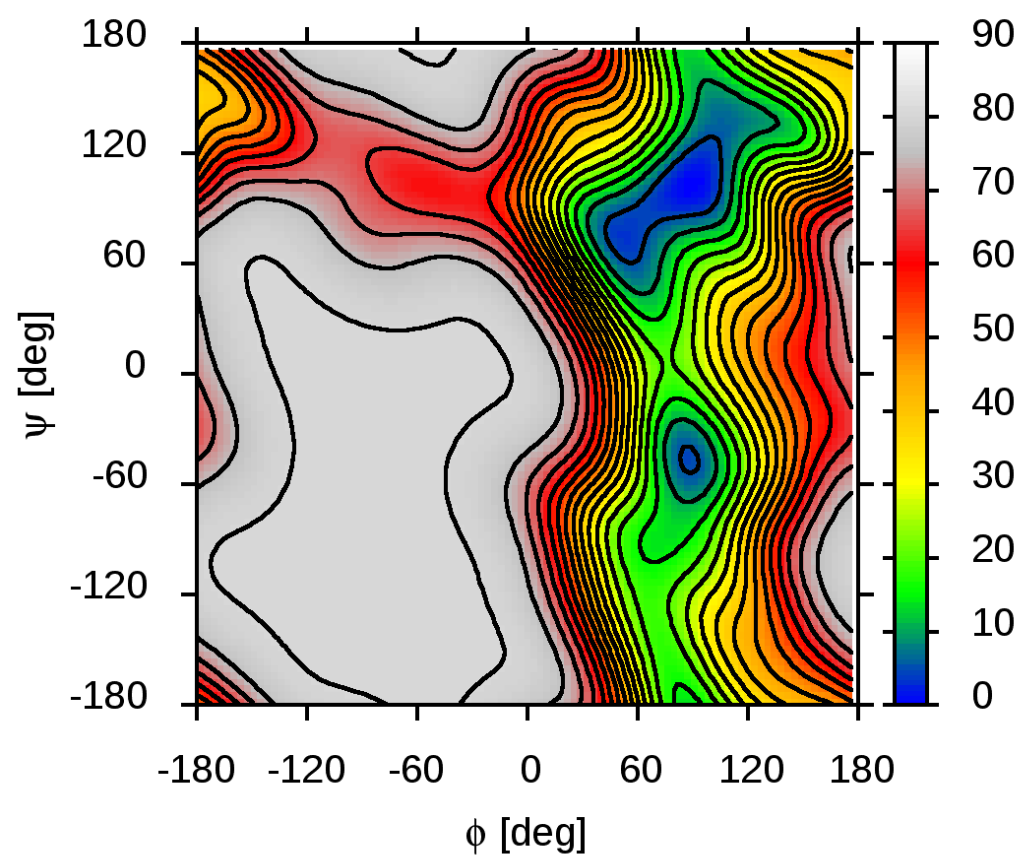

2. GlcNAc- $\alpha(1 \rightarrow 4)-\beta-G \mid c A 2 S$

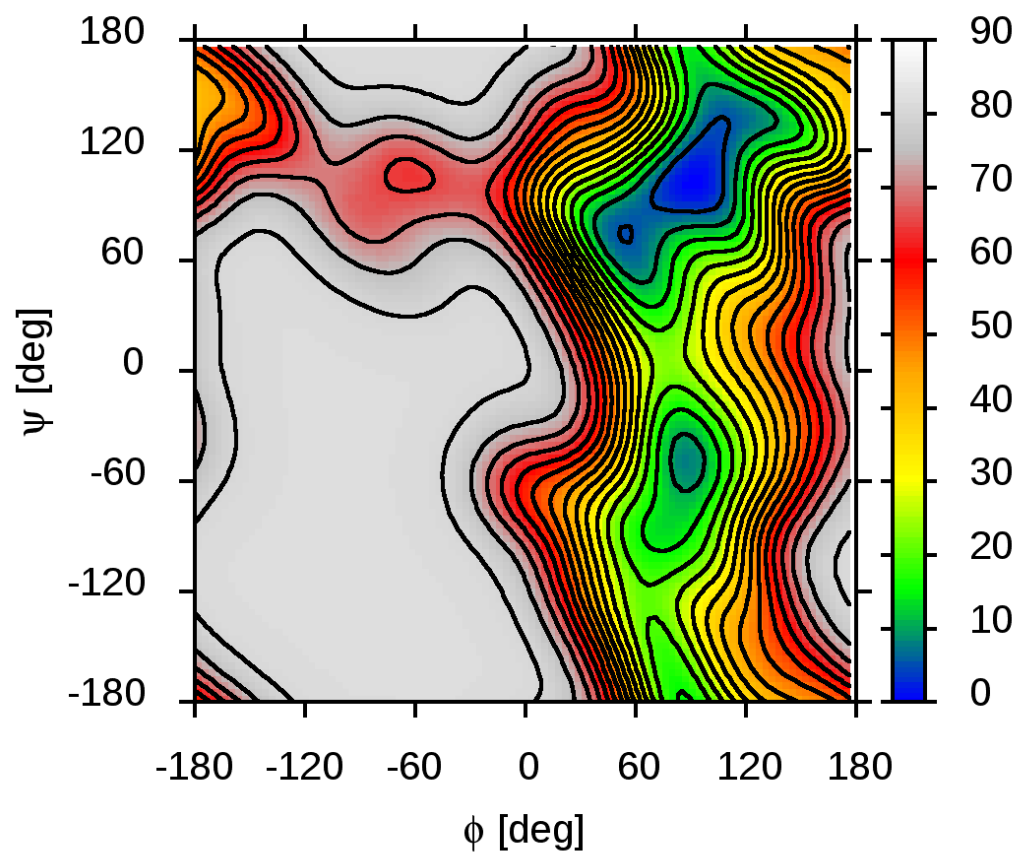


3. GlcNAc3S- $\alpha(1 \rightarrow 4)-\beta-G \mid c A$

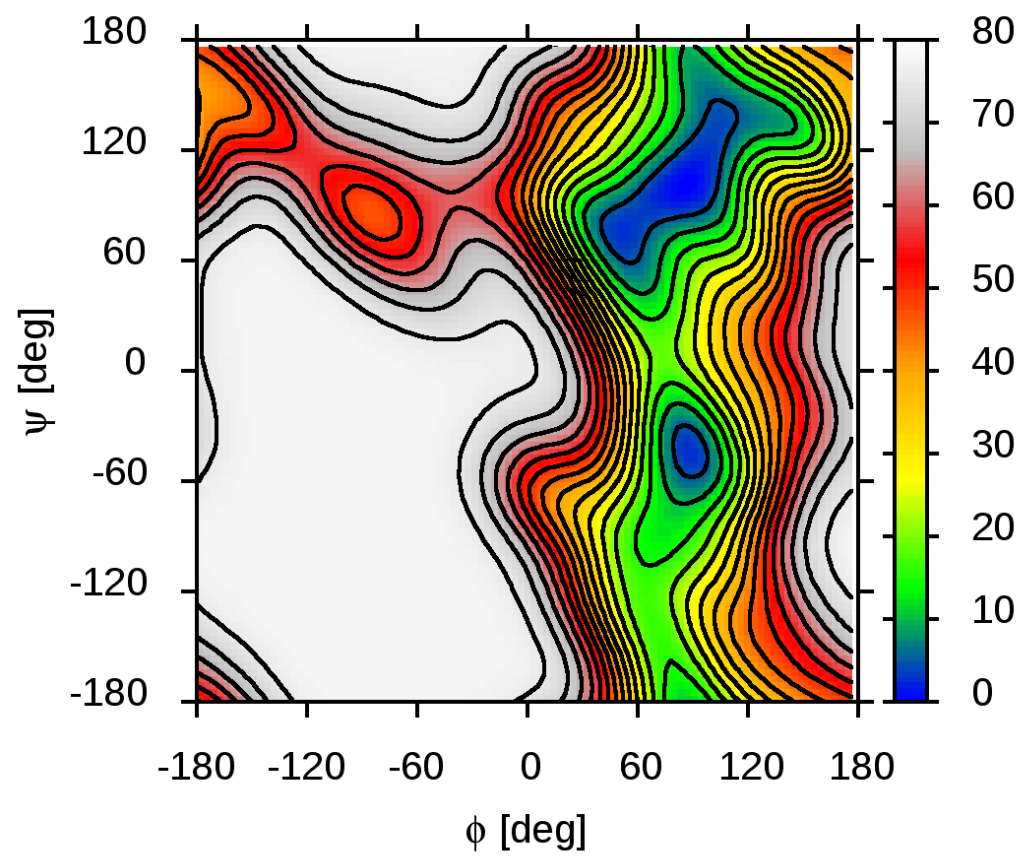

4. GlcNAc6S- $\alpha(1 \rightarrow 4)-\beta-G l c A$

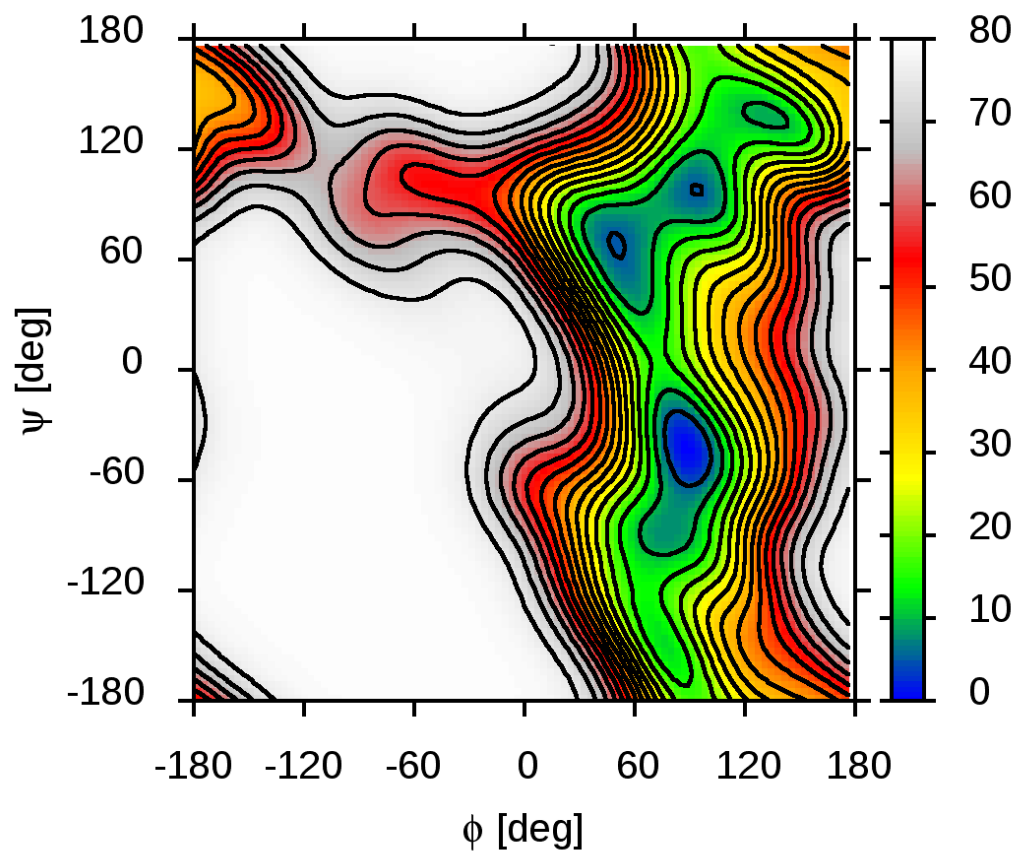


5. GlcNAc3S- $\alpha(1 \rightarrow 4)-\beta-G l c A 2 S$

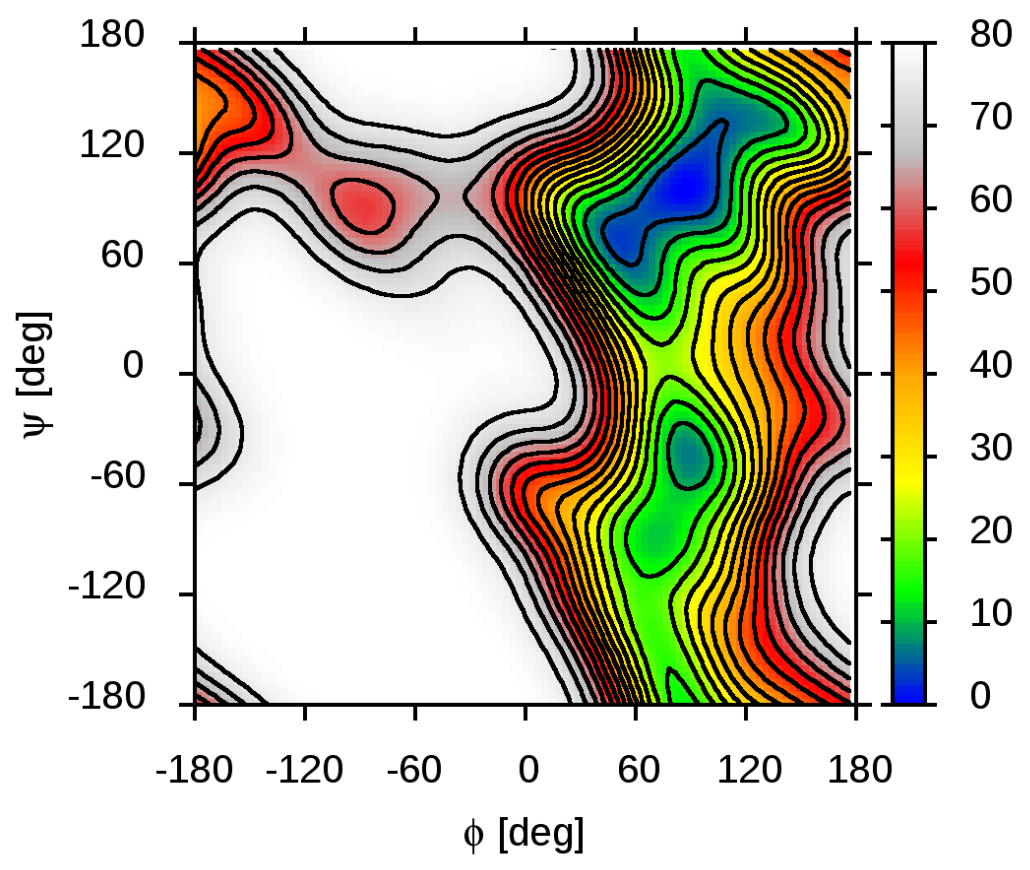

6. GlcNAc6S- $\alpha(1 \rightarrow 4)-\beta-G l c A 2 S$

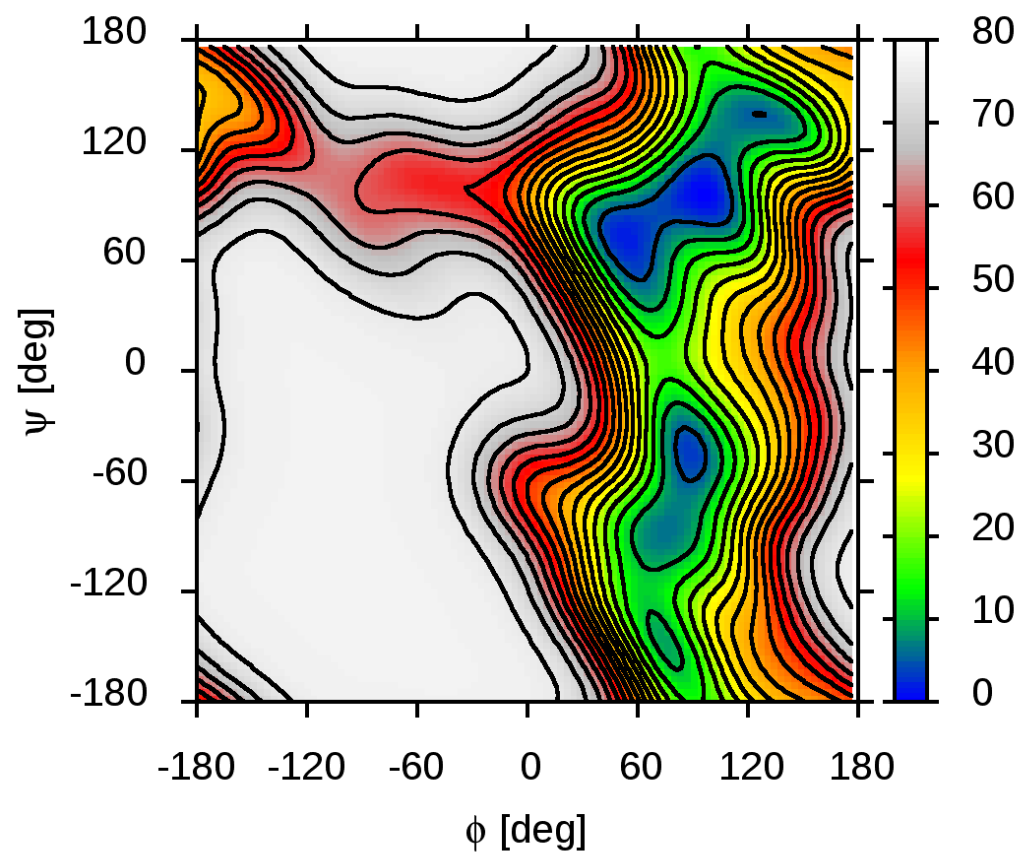


7. GlcNAc3,6S- $\alpha(1 \rightarrow 4)-\beta-G \mid c A$

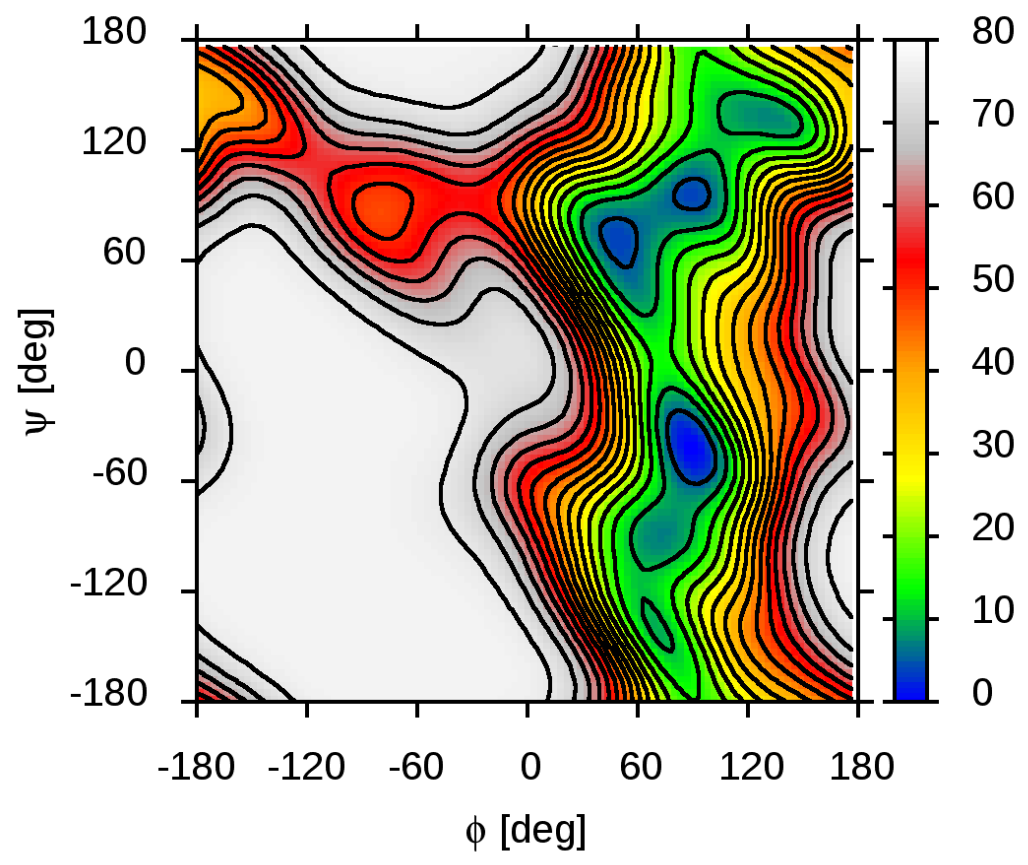

8. GlcNAc3,6S- $\alpha(1 \rightarrow 4)-\beta-G \mid c A 2 S$

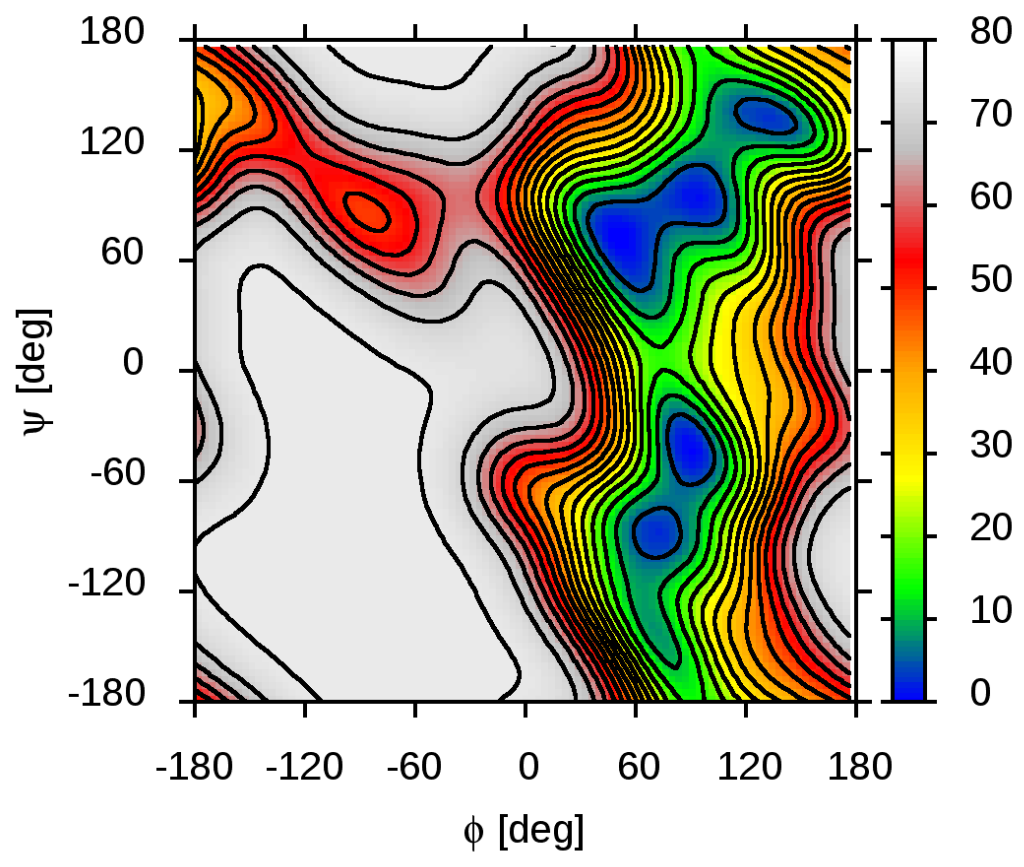


9. GlcNS- $\alpha(1 \rightarrow 4)-\beta-G \mid c A$

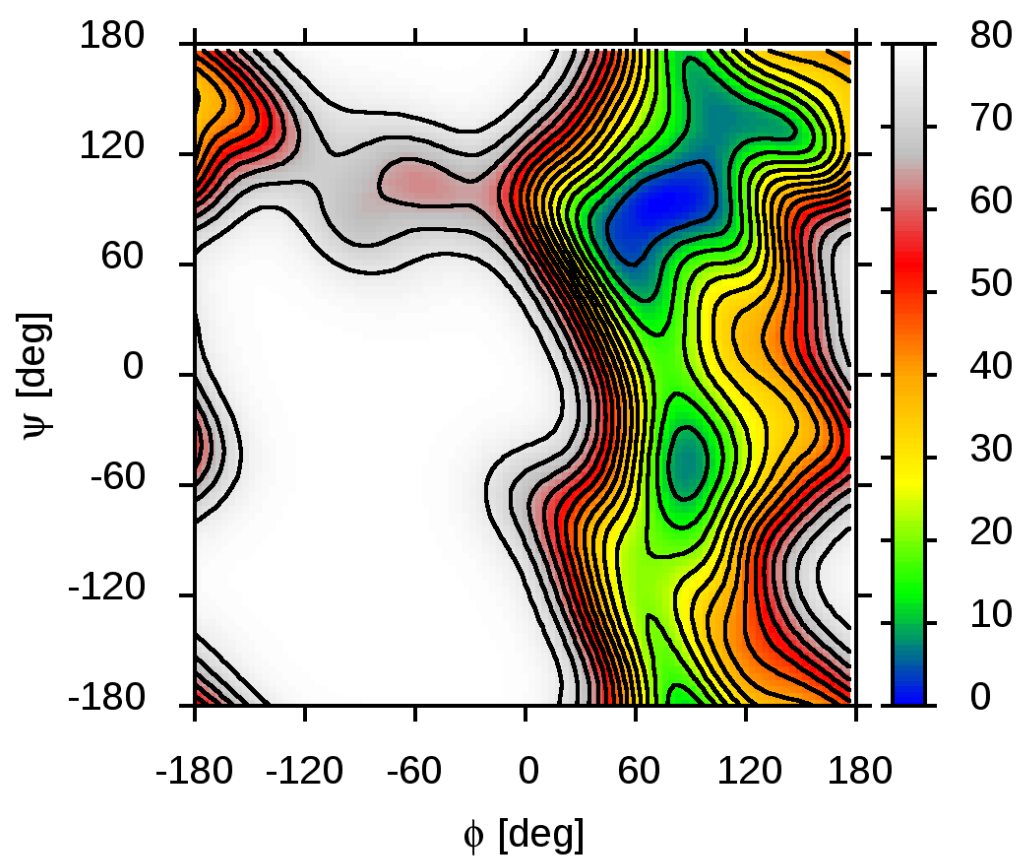

10. GlcNS- $\alpha(1 \rightarrow 4)-\beta-G \mid c A 2 S$

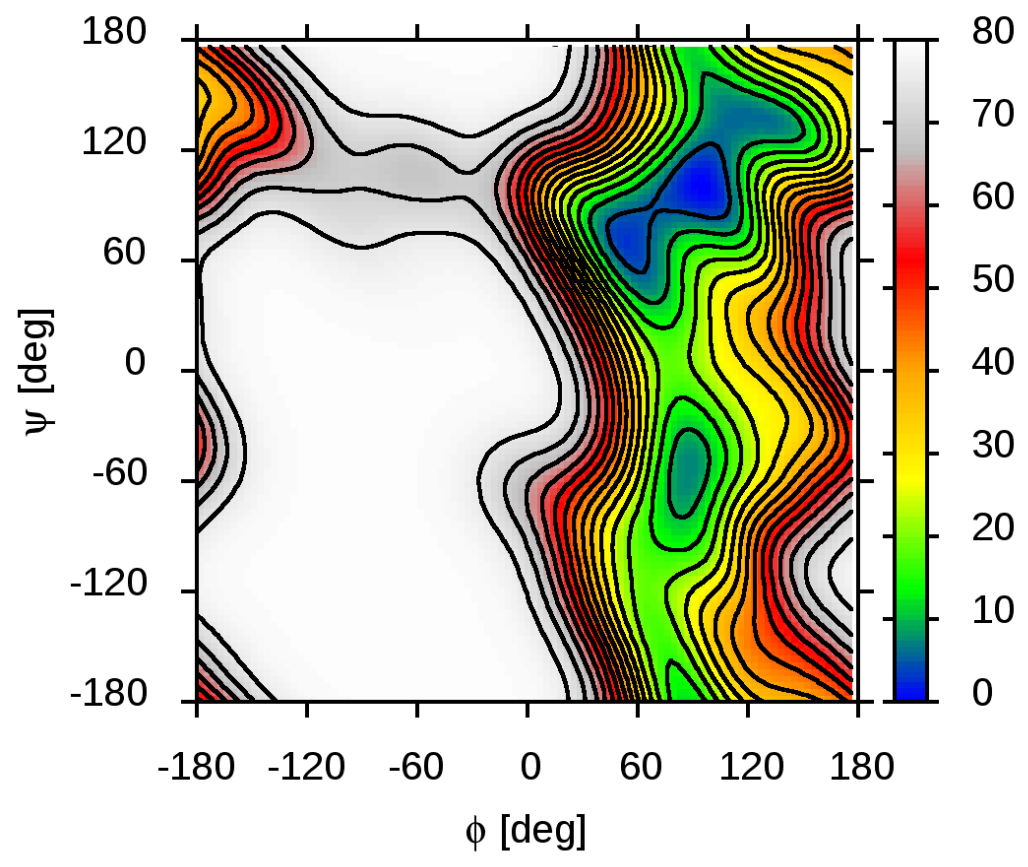


11. GlcNS3S- $\alpha(1 \rightarrow 4)-\beta-G l c A$

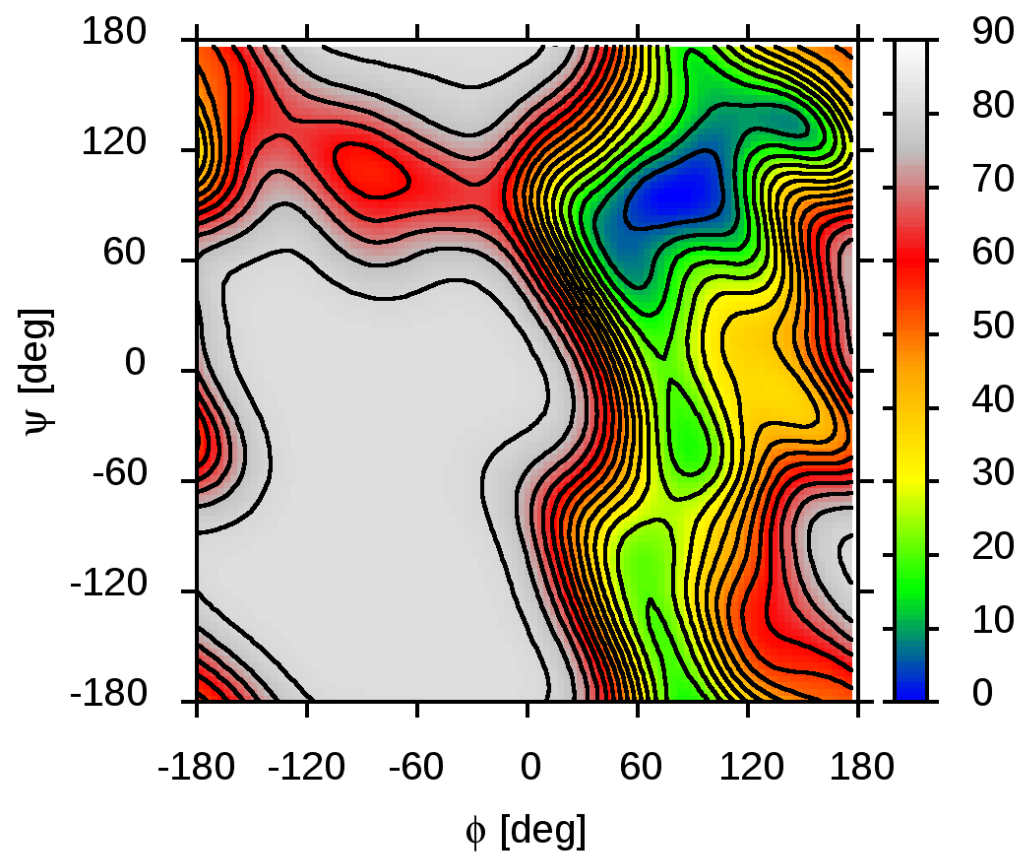

12. GlcNS6S- $\alpha(1 \rightarrow 4)-\beta-G \mid c A$

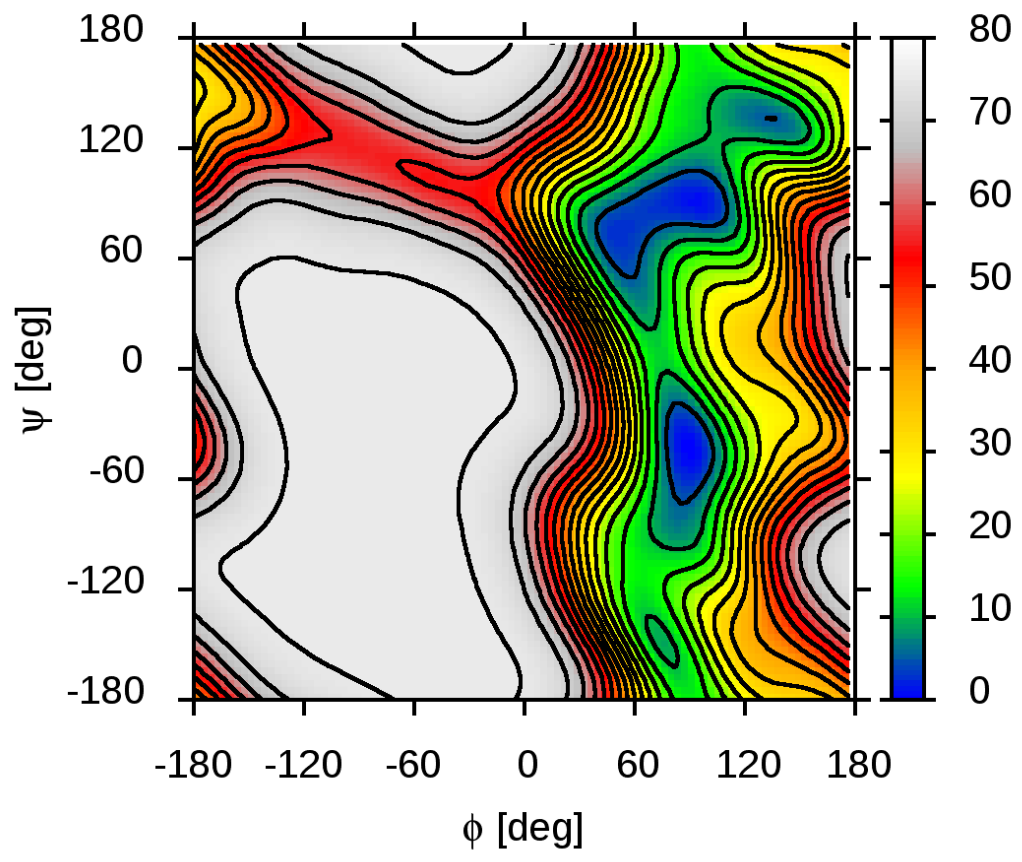


13. GlcNS3S- $\alpha(1 \rightarrow 4)-\beta-G l c A 2 S$

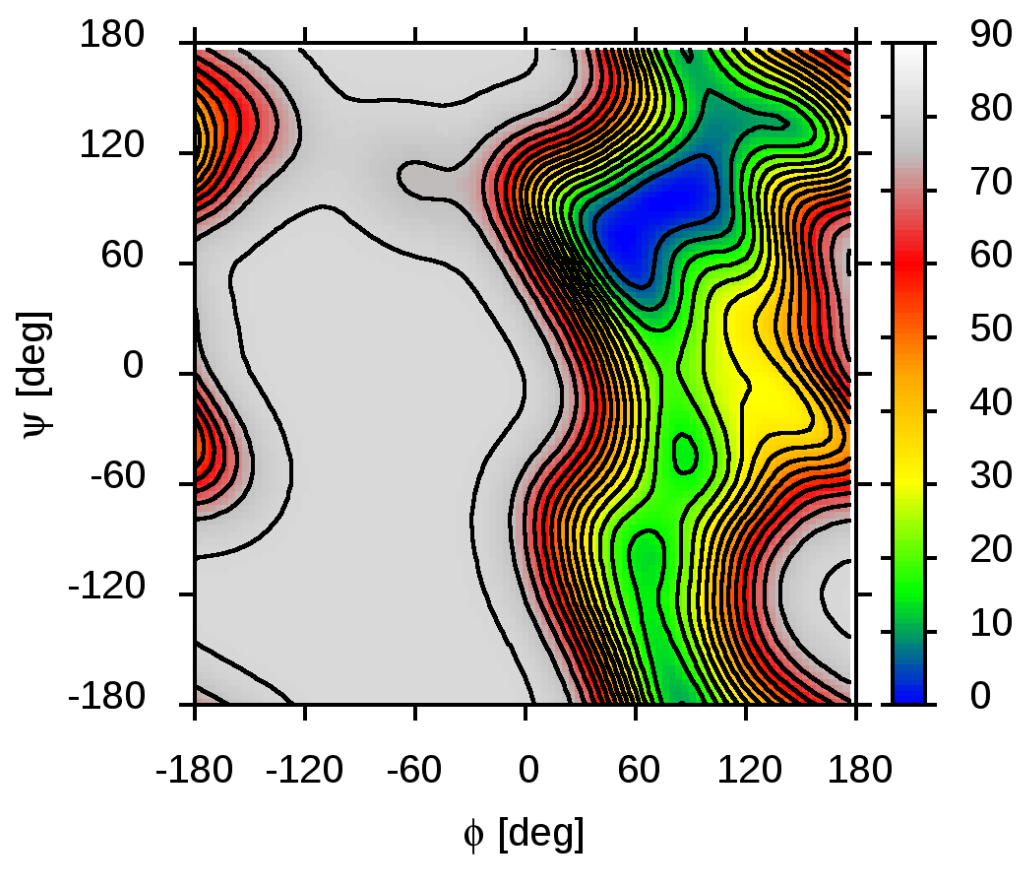

14. GlcNS6S- $\alpha(1 \rightarrow 4)-\beta$-GlcA2S

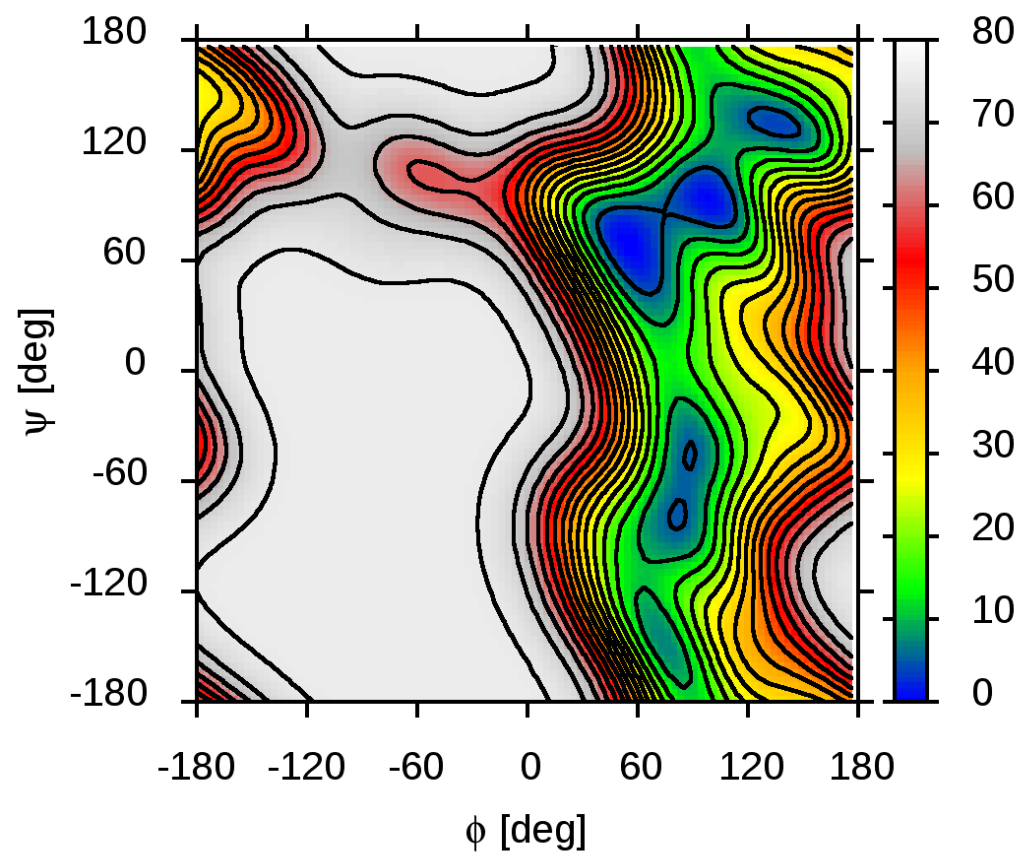


15. GlcNS3,6S- $\alpha(1 \rightarrow 4)-\beta-G l c A$

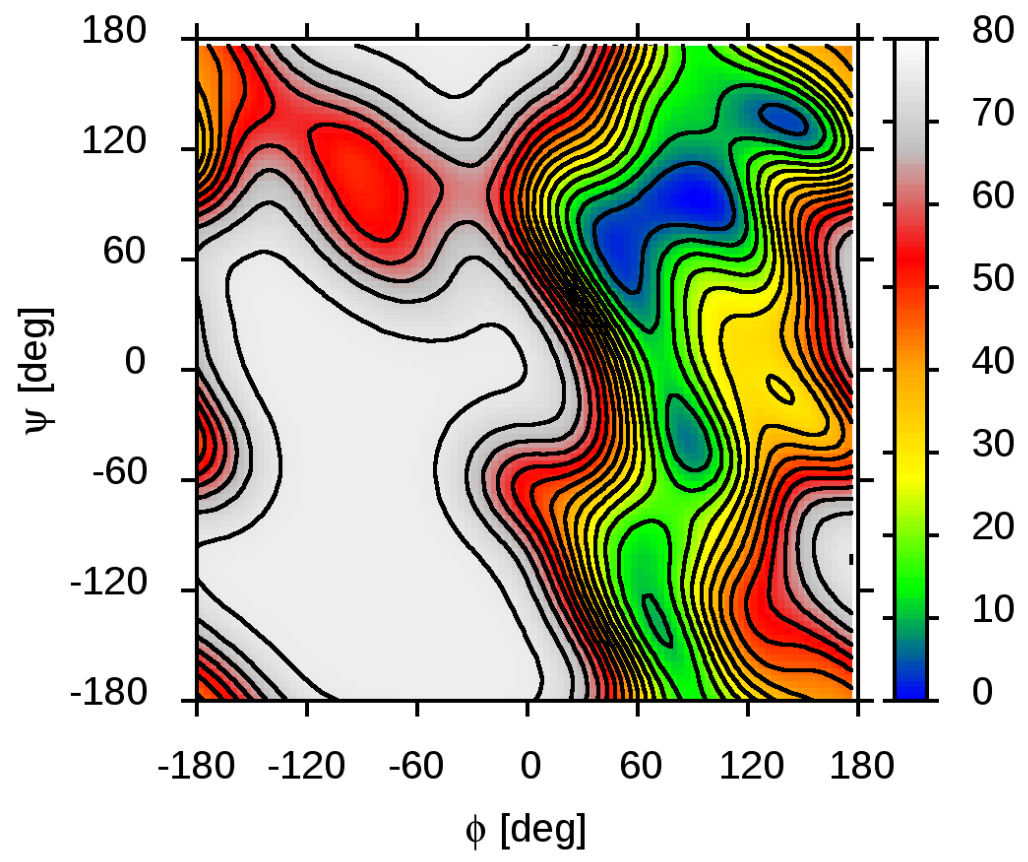

16. GlcNS3,6S- $\alpha(1 \rightarrow 4)-\beta-G \mid c A 2 S$

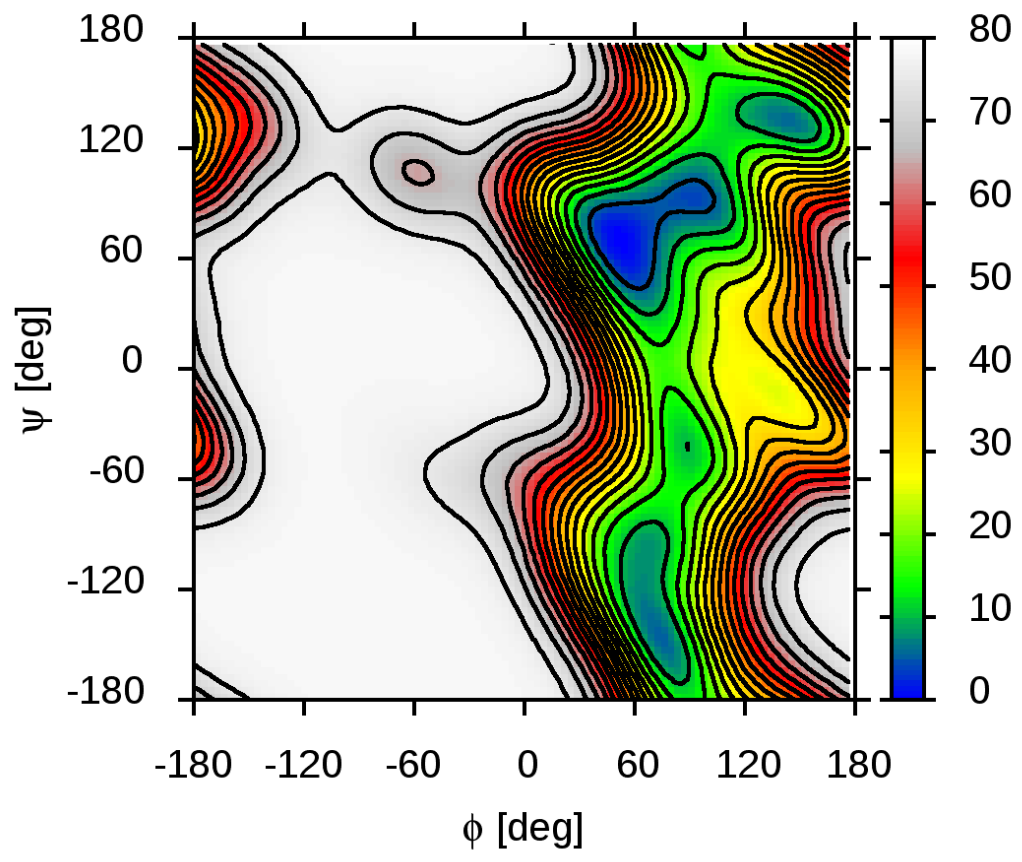


Tab. S2. The values of the $\phi$ and $\psi$ angles corresponding to the most favorable geometries of glycosidic linkages ( $3^{\text {rd }}$ column) and the 'averaged' values of $\phi$ and $\psi$, corresponding to the given threshold of the free energy $(R T, 2 R T$ or $4 R T)\left(4^{\text {th }}-6^{\text {th }}\right.$ columns). In the case of non-sulfated disaccharides, the standard deviations calculated over four independent simulations are given.

\begin{tabular}{|c|c|c|c|c|c|c|c|c|c|}
\hline \multirow{2}{*}{ GAG } & \multirow{2}{*}{ Disaccharide } & \multicolumn{2}{|c|}{ Energy minimum } & \multicolumn{2}{|c|}{ RT } & \multicolumn{2}{|c|}{ 2RT } & \multicolumn{2}{|c|}{ 4RT } \\
\hline & & $\Phi$ & $\Psi$ & $\Phi$ & $\Psi$ & $\Phi$ & $\Psi$ & $\Phi$ & $\Psi$ \\
\hline \multirow{2}{*}{ Hyaluronic acid (HA) } & $\begin{array}{l}\text { GIcA- } \beta(1 \rightarrow 3)-\beta- \\
\text { GICNAc }\end{array}$ & $-70.9 \pm 2.6$ & $-121.8 \pm 1.8$ & -70.3 & -123.1 & -73.1 & -127.0 & -75.1 & -131.7 \\
\hline & $\begin{array}{c}\text { GIcNAc- } \beta(1 \rightarrow 4)- \\
\beta-G \mid c A\end{array}$ & $-81.8 \pm 1.6$ & $129.1 \pm 1.8$ & -79.4 & 125.6 & -78.2 & 124.0 & -78.4 & 119.1 \\
\hline \multirow{7}{*}{$\begin{array}{l}\text { Chondroitin sulfate } \\
\text { (CS) }\end{array}$} & $\begin{array}{c}\text { GlcA- } \beta(1 \rightarrow 3)-\beta- \\
\text { GalNAc }\end{array}$ & $-85.5 \pm 0.0$ & $-158.2 \pm 1.8$ & -77.4 & -149.2 & -75.4 & -141.2 & -74.8 & -119.4 \\
\hline & $\begin{array}{l}\text { GlcA-B } \beta(1 \rightarrow 3)-\beta- \\
\text { GalNAc4S }\end{array}$ & -67.3 & -125.5 & -68.2 & -123.0 & -67.7 & -124.0 & -62.9 & -123.8 \\
\hline & 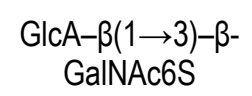 & -81.8 & -158.2 & -75.2 & -143.0 & -75.2 & -140.1 & -68.8 & -120.8 \\
\hline & $\begin{array}{c}\text { GlcA2S- } \beta(1 \rightarrow 3)- \\
\beta \text {-GalNAc }\end{array}$ & -70.9 & -121.8 & -71.6 & -126.9 & -75.0 & -133.3 & -76.4 & -112.7 \\
\hline & $\begin{array}{c}\text { GIcA2S- } \beta(1 \rightarrow 3)- \\
\beta-G a l N A c 4 S\end{array}$ & -70.9 & -114.5 & -70.8 & -115.1 & -71.8 & -116.0 & -73.3 & -117.5 \\
\hline & $\begin{array}{c}\text { GlcA2S- } \beta(1 \rightarrow 3)- \\
\beta \text {-GalNAc6S }\end{array}$ & -70.9 & -118.2 & -71.3 & -121.6 & -72.4 & -126.5 & -75.6 & -124.3 \\
\hline & $\begin{array}{l}\text { GIcA-B } \beta(1 \rightarrow 3)-\beta- \\
\text { GalNAc4,6S }\end{array}$ & -67.3 & -125.5 & -68.2 & -123.0 & -69.4 & -123.3 & -54.4 & -123.0 \\
\hline
\end{tabular}




\begin{tabular}{|c|c|c|c|c|c|c|c|c|c|}
\hline & $\begin{array}{c}\text { GlcA2S- } \beta(1 \rightarrow 3)- \\
\beta-G a I N A c 4,6 S\end{array}$ & -70.9 & -114.5 & -71.6 & -115.6 & -72.6 & -116.5 & -73.8 & -118.5 \\
\hline & $\begin{array}{c}\text { GalNAc- } \beta(1 \rightarrow 4)- \\
\beta-G \mid c A\end{array}$ & $-81.8 \pm 5.6$ & $129.1 \pm 10.0$ & -78.4 & 126.8 & -76.7 & 126.0 & -76.4 & 123.0 \\
\hline & $\begin{array}{c}\text { GalNAc- } \beta(1 \rightarrow 4)- \\
\beta-G I c A 2 S\end{array}$ & -81.8 & 136.4 & -77.6 & 132.3 & -75.9 & 131.8 & -72.7 & 123.1 \\
\hline & $\begin{array}{c}\text { GalNAc4S- } \\
\beta(1 \rightarrow 4)-\beta-G \mid c A\end{array}$ & -81.8 & 129.1 & -77.4 & 126.5 & -75.5 & 125.8 & -76.6 & 117.8 \\
\hline & $\begin{array}{c}\text { GalNAc6S- } \\
\beta(1 \rightarrow 4)-\beta-G \mid c A\end{array}$ & -74.5 & 121.8 & -74.0 & 123.0 & -74.3 & 122.6 & -75.8 & 115.6 \\
\hline & $\begin{array}{c}\text { GalNAc4S- } \\
\beta(1 \rightarrow 4)-\beta- \\
\text { GlcA2S }\end{array}$ & -85.5 & 136.4 & -83.8 & 133.8 & -81.5 & 132.1 & -78.0 & 117.2 \\
\hline & $\begin{array}{c}\text { GalNAc6S- } \\
\beta(1 \rightarrow 4)-\beta- \\
\text { GIcA2S }\end{array}$ & -81.8 & 132.7 & -78.2 & 129.3 & -77.1 & 128.9 & -74.3 & 97.0 \\
\hline & $\begin{array}{c}\text { GalNAc4,6S- } \\
\beta(1 \rightarrow 4)-\beta-G \mid c A\end{array}$ & -78.2 & 121.8 & -78.7 & 120.9 & -80.3 & 112.1 & -77.7 & 102.7 \\
\hline & $\begin{array}{l}\text { GalNAc4,6S- } \\
\beta(1 \rightarrow 4)-\beta- \\
\text { GIcA2S }\end{array}$ & -81.8 & 136.4 & -79.5 & 132.1 & -76.4 & 111.9 & -75.8 & 89.7 \\
\hline \multirow{3}{*}{ Dermatan sulfate (DS) } & $\begin{array}{c}\text { L-IdoA-a(1 } \rightarrow 3)- \\
\beta-G a I N A c\end{array}$ & $-60.0 \pm 1.8$ & $-132.7 \pm 0.0$ & -70.8 & -119.7 & -72.4 & -122.3 & -61.6 & -95.1 \\
\hline & $\begin{array}{c}\text { L-IdoA- } a(1 \rightarrow 3)- \\
\quad \beta-G a I N A c 4 S\end{array}$ & -67.3 & -147.3 & -72.7 & -107.3 & -73.4 & -107.1 & -71.8 & -143.2 \\
\hline & $\begin{array}{c}\text { L-IdoA- } a(1 \rightarrow 3)- \\
\beta-G a l N A c 6 S\end{array}$ & -56.4 & -169.1 & -69.6 & -122.0 & -71.1 & -124.9 & -64.7 & -102.5 \\
\hline
\end{tabular}




\begin{tabular}{|c|c|c|c|c|c|c|c|c|}
\hline $\begin{array}{l}\text { L-IdoA2S- } \\
a(1 \rightarrow 3)-\beta- \\
\text { GalNAc }\end{array}$ & -96.4 & -147.3 & -73.9 & -122.9 & -80.0 & -117.0 & -68.5 & -106.6 \\
\hline $\begin{array}{l}\text { L-IdoA2S- } \\
a(1 \rightarrow 3)-\beta- \\
\text { GalNAc4S }\end{array}$ & -67.3 & -147.3 & -75.8 & -108.1 & -75.9 & -108.8 & -72.4 & -122.0 \\
\hline $\begin{array}{l}\text { L-IdoA2S- } \\
a(1 \rightarrow 3)-\beta- \\
\text { GalNAc6S }\end{array}$ & -85.5 & -147.3 & -76.0 & -121.7 & -77.2 & -125.1 & -82.8 & -104.0 \\
\hline $\begin{array}{c}\text { L-IdoA-a(1 } \rightarrow 3)- \\
\beta-G a l N A c 4,6 S\end{array}$ & -67.3 & -150.9 & -72.4 & -107.1 & -72.3 & -107.1 & -74.6 & -143.6 \\
\hline $\begin{array}{l}\text { L-IdoA2S- } \\
\alpha(1 \rightarrow 3)-\beta- \\
\text { GalNAc4,6S }\end{array}$ & -60.0 & -154.5 & -74.5 & -107.3 & -75.0 & -107.2 & -59.7 & -145.7 \\
\hline $\begin{array}{c}\text { GalNAc- } \beta(1 \rightarrow 4)- \\
\alpha-L-I d o A\end{array}$ & $-63.6 \pm 1.6$ & $114.5 \pm 1.6$ & -67.9 & 120.1 & -68.6 & 119.7 & -69.3 & 117.2 \\
\hline $\begin{array}{c}\text { GalNAc- } \beta(1 \rightarrow 4)- \\
\quad \alpha-L-I d o A 2 S\end{array}$ & -89.1 & 78.2 & -76.7 & 105.6 & -76.4 & 107.5 & -75.4 & 110.5 \\
\hline $\begin{array}{c}\text { GalNAc4S- } \\
\beta(1 \rightarrow 4)-\alpha-L-I d o A\end{array}$ & -67.3 & 121.8 & -69.0 & 123.8 & -69.0 & 123.7 & -69.6 & 119.4 \\
\hline $\begin{array}{c}\text { GalNAc6S- } \\
\beta(1 \rightarrow 4)-\alpha-L-I d o A\end{array}$ & -78.2 & 129.1 & -70.9 & 118.6 & -71.6 & 115.2 & -72.0 & 113.1 \\
\hline $\begin{array}{c}\text { GalNAc4S- } \\
\beta(1 \rightarrow 4)-\alpha-L- \\
\quad \text { doA2S }\end{array}$ & -74.5 & 121.8 & -76.9 & 113.7 & -77.9 & 111.0 & -76.7 & 113.0 \\
\hline $\begin{array}{l}\text { GalNAc6S- } \\
\beta(1 \rightarrow 4)-\alpha-L- \\
\quad \text { IdoA2S }\end{array}$ & -74.5 & 118.2 & -73.6 & 117.6 & -73.6 & 117.2 & -73.8 & 116.7 \\
\hline $\begin{array}{c}\text { GalNAc4,6S- } \\
\beta(1 \rightarrow 4)-\alpha-\mathrm{L}-\text { IdoA }\end{array}$ & -67.3 & 110.9 & -70.4 & 118.6 & -71.0 & 118.4 & -72.6 & 115.1 \\
\hline
\end{tabular}




\begin{tabular}{|c|c|c|c|c|c|c|c|c|c|}
\hline & $\begin{array}{l}\text { GalNAc4,6S- } \\
\beta(1 \rightarrow 4)-\alpha-L- \\
\quad \text { IdoA2S }\end{array}$ & -67.3 & 114.5 & -68.2 & 117.3 & -67.9 & 120.0 & -68.3 & 121.5 \\
\hline \multirow{8}{*}{ Keratan sulfate (KS) } & $\begin{array}{c}\text { Gal- } \beta(1 \rightarrow 4)-\beta- \\
\text { GlcNAc }\end{array}$ & $-67.3 \pm 0.0$ & $118.2 \pm 1.6$ & -69.3 & 118.0 & -69.5 & 116.6 & -74.7 & 86.7 \\
\hline & $\begin{array}{c}\text { Gal- } \beta(1 \rightarrow 4)-\beta- \\
\text { GlcNAc6S }\end{array}$ & -70.9 & 121.8 & -70.2 & 120.1 & -71.0 & 120.1 & -73.7 & 101.5 \\
\hline & $\begin{array}{c}\text { Gal6S- } \beta(1 \rightarrow 4)- \\
\beta-G I c N A c\end{array}$ & -70.9 & 118.2 & -71.2 & 116.7 & -71.1 & 114.8 & -74.0 & 81.7 \\
\hline & $\begin{array}{c}\text { Gal6S-B(1 } \rightarrow 4)- \\
\beta-G I C N A c 6 S\end{array}$ & -70.9 & 118.2 & -71.3 & 117.8 & -70.8 & 117.6 & -72.8 & 90.0 \\
\hline & $\begin{array}{c}\operatorname{GlcNAc}-\beta(1 \rightarrow 3)- \\
\beta-G a l\end{array}$ & $-67.3 \pm 0.0$ & $-110.9 \pm 1.8$ & -75.1 & -133.5 & -76.2 & -121.1 & -76.7 & -105.9 \\
\hline & $\begin{array}{c}\text { GIcNAc- } \beta(1 \rightarrow 3)- \\
\beta-G a l 6 S\end{array}$ & -85.5 & -161.8 & -75.0 & -140.5 & -75.3 & -127.2 & -75.9 & -108.6 \\
\hline & $\begin{array}{l}\text { GlcNAc6S- } \\
\beta(1 \rightarrow 3)-\beta-G a l\end{array}$ & -85.5 & -176.4 & -83.6 & -109.0 & -78.6 & -104.5 & -79.0 & -86.8 \\
\hline & $\begin{array}{c}\text { GlcNAc6S- } \\
\beta(1 \rightarrow 3)-\beta-G a l 6 S\end{array}$ & -85.5 & -172.7 & -82.3 & -124.3 & -77.6 & -108.2 & -78.8 & -81.0 \\
\hline \multirow{3}{*}{$\begin{array}{l}\text { Heparin/Heparan } \\
\text { sulfate (HP/HS) }\end{array}$} & $\begin{array}{c}\text { L-IdoA-a }(1 \rightarrow 4)- \\
a-G I c N A c\end{array}$ & $-78.2 \pm 1.6$ & $125.5 \pm 0.0$ & -85.7 & 98.6 & -88.0 & 96.0 & -87.8 & 62.1 \\
\hline & $\begin{array}{c}\text { L-IdoA-a }(1 \rightarrow 4)- \\
\text { a-GlcNAc3S }\end{array}$ & -78.2 & 143.6 & -75.1 & 141.4 & -78.8 & 80.5 & -78.5 & 52.7 \\
\hline & 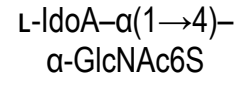 & -81.8 & 129.1 & -80.7 & 126.3 & -84.2 & 109.3 & -87.7 & 103.3 \\
\hline
\end{tabular}




\begin{tabular}{|c|c|c|c|c|c|c|c|c|}
\hline $\begin{array}{l}\text { L-IdoA2S- } \\
\mathrm{a}(1 \rightarrow 4)-\mathrm{a}- \\
\quad \text { GIcNAc }\end{array}$ & -89.1 & 81.8 & -87.6 & 96.3 & -87.8 & 98.1 & -88.4 & 96.5 \\
\hline $\begin{array}{l}\text { L-IdoA2S- } \\
\mathrm{a}(1 \rightarrow 4)-\alpha- \\
\text { GlcNAc3S }\end{array}$ & -67.3 & 129.1 & -70.5 & 131.9 & -70.8 & 132.1 & -71.6 & 131.1 \\
\hline $\begin{array}{l}\text { L-IdoA2S- } \\
\mathrm{a}(1 \rightarrow 4)-\mathrm{a}- \\
\text { GlcNAc6S }\end{array}$ & -81.8 & 129.1 & -86.4 & 101.4 & -87.3 & 100.7 & -87.4 & 99.5 \\
\hline $\begin{array}{c}\text { L-IdoA-a(1 } \rightarrow 4)- \\
a-G I c N A c 3,6 S\end{array}$ & -67.3 & 136.4 & -68.4 & 135.6 & -67.6 & 135.1 & -66.5 & 135.2 \\
\hline $\begin{array}{l}\text { L-IdoA2S- } \\
a(1 \rightarrow 4)-\alpha- \\
\text { GlcNAc3,6S }\end{array}$ & -74.5 & 140.0 & -74.6 & 138.7 & -73.9 & 138.5 & -74.4 & 124.0 \\
\hline $\begin{array}{l}\text { L-IdoA-a(1 } \rightarrow 4)- \\
\quad a-G I c N S\end{array}$ & -78.2 & 125.5 & -84.0 & 101.0 & -84.8 & 98.8 & -86.2 & 89.2 \\
\hline $\begin{array}{l}\text { L-IdoA-a }(1 \rightarrow 4)- \\
\text { a-GICNS3S }\end{array}$ & -81.8 & 140.0 & -78.7 & 137.4 & -79.6 & 102.4 & -87.7 & 70.4 \\
\hline $\begin{array}{l}\text { L-IdoA-a(1 } \rightarrow 4)- \\
\quad a-G I c N S 6 S\end{array}$ & -81.8 & 129.1 & -81.6 & 126.6 & -85.2 & 110.8 & -88.1 & 105.0 \\
\hline$\underset{\mathrm{L}}{\mathrm{L}-\mathrm{IdoA} 2 \mathrm{(} \rightarrow 4)-\mathrm{a}-\mathrm{GlcNS}}$ & -89.1 & 78.2 & -88.3 & 94.1 & -88.4 & 98.3 & -90.8 & 80.6 \\
\hline $\begin{array}{l}\text { L-IdoA2S- } \\
\mathrm{a}(1 \rightarrow 4)-\mathrm{a}- \\
\text { GIcNS3S }\end{array}$ & -85.5 & 140.0 & -82.7 & 138.8 & -81.8 & 138.3 & -81.6 & 56.9 \\
\hline $\begin{array}{l}\text { L-IdoA2S- } \\
\mathrm{a}(1 \rightarrow 4)-\mathrm{a}- \\
\text { GIcNS6S }\end{array}$ & -85.5 & 129.1 & -88.8 & 101.0 & -89.3 & 100.0 & -90.5 & 100.3 \\
\hline $\begin{array}{c}\text { L-IdoA-a }(1 \rightarrow 4)- \\
\text { a-GlcNS3,6S }\end{array}$ & -81.8 & 140.0 & -80.6 & 140.7 & -78.5 & 139.4 & -81.0 & 132.4 \\
\hline
\end{tabular}




\begin{tabular}{|c|c|c|c|c|c|c|c|c|}
\hline $\begin{array}{l}\text { L-IdoA2S- } \\
a(1 \rightarrow 4)-a- \\
\text { GlcNS3,6S }\end{array}$ & -89.1 & 143.6 & -83.8 & 144.0 & -84.9 & 139.4 & -91.9 & 118.1 \\
\hline $\begin{array}{c}\text { GIcNAc- } a(1 \rightarrow 4)- \\
a-L-I d o A\end{array}$ & $74.5 \pm 1.6$ & $85.5 \pm 1.6$ & 70.4 & 81.4 & 70.2 & 80.6 & 70.8 & 81.1 \\
\hline $\begin{array}{c}\text { GIcNAc-a }(1 \rightarrow 4)- \\
a-\text {-LIdoA2S }\end{array}$ & 60.0 & 67.3 & 64.9 & 73.8 & 66.1 & 74.2 & 68.8 & 75.6 \\
\hline $\begin{array}{c}\text { GlcNAc3S- } \\
a(1 \rightarrow 4)-a-L-I d o A\end{array}$ & 74.5 & 89.1 & 69.5 & 84.7 & 68.4 & 83.3 & 69.3 & 83.3 \\
\hline $\begin{array}{c}\text { GlcNAc6S- } \\
a(1 \rightarrow 4)-a-L-I d o A\end{array}$ & 60.0 & 70.9 & 62.4 & 74.9 & 64.9 & 76.3 & 66.2 & 78.0 \\
\hline $\begin{array}{l}\text { GlcNAc3S- } \\
\mathrm{a}(1 \rightarrow 4)-\mathrm{a}-\mathrm{L}- \\
\quad \text { IdoA2S }\end{array}$ & 52.7 & 60.0 & 59.2 & 70.5 & 62.8 & 75.6 & 64.5 & 76.7 \\
\hline $\begin{array}{l}\text { GlcNAc6S- } \\
a(1 \rightarrow 4)-a-L- \\
\text { IdoA2S }\end{array}$ & 60.0 & 67.3 & 58.8 & 67.3 & 60.3 & 67.6 & 64.1 & 70.1 \\
\hline 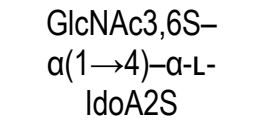 & 49.1 & 63.6 & 50.9 & 63.3 & 51.2 & 63.8 & 56.5 & 68.8 \\
\hline $\begin{array}{c}\text { GlcNAc3,6S- } \\
a(1 \rightarrow 4)-a-L-I d o A\end{array}$ & 52.7 & 70.9 & 53.4 & 70.4 & 61.3 & 76.0 & 65.2 & 79.5 \\
\hline $\begin{array}{l}\text { GlcNS-a }(1 \rightarrow 4)- \\
\quad a-L-I d o A\end{array}$ & 78.2 & 89.1 & 76.6 & 87.7 & 74.9 & 85.6 & 74.0 & 84.0 \\
\hline $\begin{array}{c}\text { GlcNS-a }(1 \rightarrow 4)- \\
\text { a-L-IdoA2S }\end{array}$ & 81.8 & 92.7 & 84.1 & 92.8 & 82.4 & 91.4 & 78.1 & 87.0 \\
\hline $\begin{array}{c}\text { GlcNS3S- } \\
a(1 \rightarrow 4)-a-L-I d o A\end{array}$ & 81.8 & 92.7 & 80.9 & 91.1 & 77.8 & 88.1 & 81.2 & 89.6 \\
\hline
\end{tabular}




\begin{tabular}{|c|c|c|c|c|c|c|c|c|}
\hline $\begin{array}{c}\text { GIcNS6S- } \\
a(1 \rightarrow 4)-a-L-I d o A\end{array}$ & 60.0 & 74.5 & 65.8 & 79.6 & 66.9 & 80.3 & 67.9 & 81.4 \\
\hline $\begin{array}{l}\text { GlcNS3S- } \\
a(1 \rightarrow 4)-a-L- \\
\quad \text { IdoA2S }\end{array}$ & 67.3 & 78.2 & 69.7 & 79.0 & 71.8 & 80.3 & 73.6 & 80.6 \\
\hline $\begin{array}{l}\text { GlcNS6S- } \\
a(1 \rightarrow 4)-a-L- \\
\text { IdoA2S }\end{array}$ & 89.1 & 92.7 & 81.5 & 85.5 & 74.0 & 80.8 & 84.1 & 85.8 \\
\hline $\begin{array}{c}\text { GlcNS3,6S- } \\
a(1 \rightarrow 4)-a-\text {-L-IdoA }\end{array}$ & 56.4 & 70.9 & 83.0 & 86.6 & 90.1 & 90.7 & 94.8 & 93.8 \\
\hline $\begin{array}{l}\text { GlcNS3,6S- } \\
a(1 \rightarrow 4)-\alpha-L- \\
\quad \operatorname{ldoA} 2 S\end{array}$ & 158.2 & 121.8 & 117.5 & 101.4 & 95.6 & 90.8 & 92.6 & 89.1 \\
\hline $\begin{array}{c}\text { GlcA- } \beta(1 \rightarrow 4)-\alpha- \\
\text { GlcNAc }\end{array}$ & $-74.5 \pm 1.6$ & $121.8 \pm 0.0$ & -73.4 & 119.9 & -73.4 & 117.6 & -77.5 & 87.6 \\
\hline $\begin{array}{l}\text { GlcA- } \beta(1 \rightarrow 4)-\alpha- \\
\quad \text { GlcNAc3S }\end{array}$ & -74.5 & 129.1 & -74.0 & 129.2 & -72.2 & 128.9 & -73.6 & 54.9 \\
\hline $\begin{array}{l}\text { GlcA- } \beta(1 \rightarrow 4)-\alpha- \\
\text { GlcNAc6S }\end{array}$ & -74.5 & 121.8 & -74.8 & 119.8 & -75.0 & 119.5 & -76.7 & 112.4 \\
\hline $\begin{array}{c}\text { GlcA2S- } \beta(1 \rightarrow 4)- \\
a-G I c N A c\end{array}$ & -78.2 & 118.2 & -77.7 & 115.2 & -79.6 & 110.2 & -80.8 & 105.4 \\
\hline $\begin{array}{c}\text { GlcA2S- } \beta(1 \rightarrow 4)- \\
\text { a-GIcNAc3S }\end{array}$ & -81.8 & 129.1 & -80.6 & 128.6 & -80.5 & 128.0 & -79.2 & 128.6 \\
\hline $\begin{array}{c}\text { GlcA2S- } \beta(1 \rightarrow 4)- \\
\text { a-GIcNAc6S }\end{array}$ & -78.2 & 121.8 & -76.0 & 121.2 & -76.5 & 120.9 & -77.2 & 117.2 \\
\hline $\begin{array}{l}\text { GlcA- } \beta(1 \rightarrow 4)-\alpha- \\
\text { GlcNAc3,6S }\end{array}$ & -74.5 & 129.1 & -74.9 & 129.3 & -73.7 & 129.9 & -70.8 & 130.6 \\
\hline
\end{tabular}




\begin{tabular}{|c|c|c|c|c|c|c|c|c|}
\hline 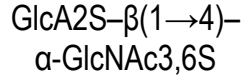 & -81.8 & 129.1 & -80.4 & 130.0 & -81.0 & 130.5 & -79.8 & 131.4 \\
\hline $\begin{array}{c}\text { GlcA- } \beta(1 \rightarrow 4)-\alpha- \\
\text { GlcNS }\end{array}$ & -74.5 & 118.2 & -75.3 & 116.7 & -78.3 & 109.9 & -79.7 & 97.3 \\
\hline $\begin{array}{c}\text { GlcA- } \beta(1 \rightarrow 4)-\alpha- \\
\text { GlcNS3S }\end{array}$ & -78.2 & 129.1 & -77.0 & 127.7 & -75.6 & 127.7 & -76.1 & 60.4 \\
\hline $\begin{array}{c}\text { GlcA- } \beta(1 \rightarrow 4)-\alpha- \\
\text { GlcNS6S }\end{array}$ & -74.5 & 118.2 & -73.7 & 117.2 & -73.2 & 116.7 & -77.4 & 109.1 \\
\hline $\begin{array}{c}\text { GlcA2S- } \beta(1 \rightarrow 4)- \\
\alpha-G I c N S\end{array}$ & -78.2 & 118.2 & -76.7 & 115.5 & -78.4 & 111.9 & -80.1 & 105.2 \\
\hline $\begin{array}{c}\text { GIcA2S- } \beta(1 \rightarrow 4)- \\
a-G I c N S 3 S\end{array}$ & -81.8 & 125.5 & -82.3 & 126.1 & -81.6 & 126.1 & -81.0 & 70.6 \\
\hline $\begin{array}{c}\text { GlcA2S- } \beta(1 \rightarrow 4)- \\
\text { a-GlcNS6S }\end{array}$ & -74.5 & 121.8 & -75.5 & 121.7 & -75.1 & 121.1 & -76.2 & 118.2 \\
\hline $\begin{array}{c}\text { GlcA- } \beta(1 \rightarrow 4)-\alpha- \\
\text { GIcNS3,6S }\end{array}$ & -74.5 & 129.1 & -73.7 & 128.6 & -72.6 & 128.8 & -71.3 & 108.8 \\
\hline $\begin{array}{c}\text { GlcA2S- } \beta(1 \rightarrow 4)- \\
\alpha-G l c N S 3,6 S\end{array}$ & -81.8 & 121.8 & -82.5 & 121.5 & -81.9 & 121.3 & -80.4 & 122.4 \\
\hline $\begin{array}{c}\text { GIcNAc-a(1 } \rightarrow 4)- \\
\beta-G \mid c A\end{array}$ & $89.1 \pm 0.0$ & $103.6 \pm 0.0$ & 89.0 & 102.6 & 78.7 & 86.4 & 83.1 & 77.0 \\
\hline 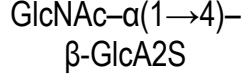 & 92.7 & 103.6 & 91.6 & 103.7 & 91.5 & 105.0 & 86.6 & 88.9 \\
\hline $\begin{array}{l}\text { GlcNAc3S- } \\
\mathrm{a}(1 \rightarrow 4)-\beta-G \mid c A\end{array}$ & 85.5 & 100.0 & 85.6 & 99.4 & 80.6 & 79.2 & 85.2 & 79.4 \\
\hline
\end{tabular}




\begin{tabular}{|c|c|c|c|c|c|c|c|c|}
\hline $\begin{array}{c}\text { GlcNAc6S- } \\
a(1 \rightarrow 4)-\beta-G \mid c A\end{array}$ & 89.1 & -41.8 & 88.4 & -41.9 & 84.7 & -25.3 & 79.2 & 21.0 \\
\hline $\begin{array}{c}\text { GlcNAc3S- } \\
a(1 \rightarrow 4)-\beta- \\
\text { GlcA2S }\end{array}$ & 85.5 & 100.0 & 86.4 & 102.4 & 79.0 & 96.2 & 85.3 & 86.1 \\
\hline $\begin{array}{c}\text { GlcNAc6S- } \\
\alpha(1 \rightarrow 4)-\beta- \\
\text { GIcA2S }\end{array}$ & 96.4 & 96.4 & 82.4 & 89.3 & 78.6 & 66.4 & 85.0 & 40.0 \\
\hline $\begin{array}{c}\text { GlcNAc3,6S- } \\
a(1 \rightarrow 4)-\beta-G \mid c A\end{array}$ & 89.1 & -41.8 & 89.1 & -41.8 & 78.2 & 15.3 & 80.6 & 26.1 \\
\hline $\begin{array}{c}\text { GlcNAc3,6S- } \\
a(1 \rightarrow 4)-\beta- \\
\text { GIcA2S }\end{array}$ & 52.7 & 70.9 & 73.2 & 44.3 & 80.2 & 43.0 & 83.2 & 27.8 \\
\hline $\begin{array}{c}\text { GIcNS-a }(1 \rightarrow 4)- \\
\beta-G \mid c A\end{array}$ & 74.5 & 92.7 & 75.4 & 92.4 & 74.3 & 90.5 & 85.4 & 87.8 \\
\hline $\begin{array}{c}\text { GIcNS-a }(1 \rightarrow 4)- \\
\beta-G I c A 2 S\end{array}$ & 96.4 & 100.0 & 95.1 & 101.2 & 80.0 & 91.0 & 88.4 & 81.1 \\
\hline $\begin{array}{c}\text { GIcNS3S- } \\
a(1 \rightarrow 4)-\beta-G \mid c A\end{array}$ & 81.8 & 96.4 & 82.0 & 96.2 & 82.0 & 96.7 & 85.5 & 99.2 \\
\hline $\begin{array}{c}\text { GlcNS6S- } \\
a(1 \rightarrow 4)-\beta-G \mid c A\end{array}$ & 89.1 & -41.8 & 90.4 & 20.8 & 79.2 & 40.2 & 85.4 & 42.5 \\
\hline $\begin{array}{c}\text { GlcNS3S- } \\
a(1 \rightarrow 4)-\beta- \\
\text { GlcA2S }\end{array}$ & 78.2 & 96.4 & 69.1 & 85.4 & 71.2 & 85.8 & 78.8 & 92.8 \\
\hline $\begin{array}{c}\text { GlcNS6S- } \\
\alpha(1 \rightarrow 4)-\beta- \\
\text { GlcA2S }\end{array}$ & 56.4 & 70.9 & 72.9 & 78.7 & 82.5 & 75.5 & 87.3 & 36.1 \\
\hline $\begin{array}{c}\text { GlcNS3,6S- } \\
\alpha(1 \rightarrow 4)-\beta-G \mid c A\end{array}$ & 92.7 & 92.7 & 82.2 & 88.4 & 80.0 & 88.6 & 85.9 & 77.2 \\
\hline
\end{tabular}




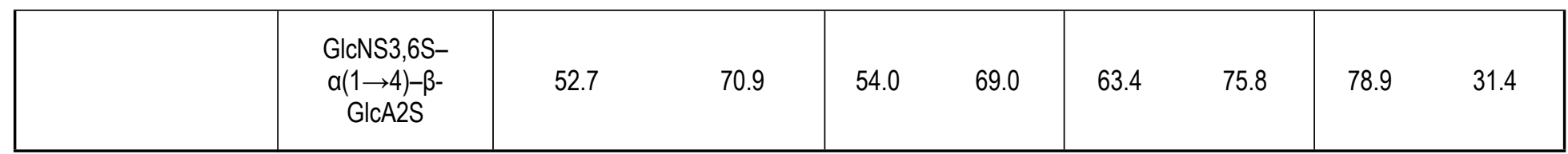


Tab. S3. The occurrences of hydrogen bonds (per frame of MD trajectory) between two monosaccharides that create given GAG disaccharide ( $4^{\text {th }}$ column) and the flexibility factors $F$ (number of points on the $\phi v s$. $\psi$ grid (of the dimensions of $99^{*} 99$ ) for which the energy level does not exceed the global minimum by more than the arbitrarily accepted threshold) ( $5^{\text {th }}-7^{\text {th }}$ columns). In the case of non-sulfated disaccharides, the standard deviations calculated over four independent simulations are given.

\begin{tabular}{|c|c|c|c|c|c|c|}
\hline \multirow{2}{*}{ GAG } & \multirow{2}{*}{ Linkage } & \multirow{2}{*}{ Disaccharide } & \multirow{2}{*}{$\begin{array}{c}\text { Hydrogen } \\
\text { bonds }\end{array}$} & \multicolumn{3}{|c|}{ Flexibility factors $F$} \\
\hline & & & & 1RT & 2RT & 4RT \\
\hline \multirow{2}{*}{$\begin{array}{l}\text { Hyaluronic acid } \\
(\mathrm{HA})\end{array}$} & $\begin{array}{c}\beta-G I c A-\beta(1 \rightarrow 3)-\beta- \\
\text { GIcNAc }\end{array}$ & GlcA- $\beta(1 \rightarrow 3)-\beta-G \mid c N A c$ & 0.711 & $52 \pm 4$ & $119 \pm 9$ & $267 \pm 17$ \\
\hline & $\begin{array}{c}\beta-G I c N A c-\beta(1 \rightarrow 4)- \\
\beta-G I c A\end{array}$ & GlcNAc- $\beta(1 \rightarrow 4)-\beta-G \mid c A$ & 0.863 & $57 \pm 6$ & $113 \pm 12$ & $260 \pm 22$ \\
\hline \multirow{8}{*}{$\begin{array}{l}\text { Chondroitin } \\
\text { sulfate (CS) }\end{array}$} & \multirow{8}{*}{$\begin{array}{c}\text { GIcA2R }^{1}-\beta(1 \rightarrow 3)-\beta- \\
\text { GalNAc4,6R }\end{array}$} & GlcA- $\beta(1 \rightarrow 3)-\beta$-GalNAc & 0.264 & $98 \pm 7$ & $189 \pm 5$ & $356 \pm 7$ \\
\hline & & $\begin{array}{l}\text { GlcA- } \beta(1 \rightarrow 3)-\beta- \\
\text { GalNAc4S }\end{array}$ & 0.202 & 53 & 102 & 218 \\
\hline & & $\begin{array}{l}\text { GlcA- } \beta(1 \rightarrow 3)-\beta- \\
\text { GalNAc6S }\end{array}$ & 0.255 & 128 & 225 & 422 \\
\hline & & $\begin{array}{c}\text { GlcA2S- } \beta(1 \rightarrow 3)-\beta- \\
\text { GalNAc }\end{array}$ & 0.069 & 71 & 184 & 356 \\
\hline & & $\begin{array}{l}\text { GIcA2S- } \beta(1 \rightarrow 3)-\beta- \\
\quad \text { GalNAc4S }\end{array}$ & 0.008 & 48 & 97 & 200 \\
\hline & & $\begin{array}{c}\text { GlcA2S- } \beta(1 \rightarrow 3)-\beta- \\
\text { GalNAc6S }\end{array}$ & 0.050 & 55 & 129 & 305 \\
\hline & & $\begin{array}{l}\text { GlcA- } \beta(1 \rightarrow 3)-\beta- \\
\text { GalNAc4,6S }\end{array}$ & 0.245 & 53 & 110 & 255 \\
\hline & & $\begin{array}{l}\text { GlcA2S- } \beta(1 \rightarrow 3)-\beta- \\
\text { GalNAc4,6S }\end{array}$ & 0.032 & 51 & 100 & 201 \\
\hline
\end{tabular}




\begin{tabular}{|c|c|c|c|c|c|c|}
\hline & & GalNAc- $\beta(1 \rightarrow 4)-\beta-G \mid c A$ & 0.834 & $51 \pm 1$ & $105 \pm 3$ & $233 \pm 9$ \\
\hline & & $\begin{array}{c}\text { GalNAc- } \beta(1 \rightarrow 4)-\beta- \\
\text { GICA2S }\end{array}$ & 0.710 & 54 & 113 & 245 \\
\hline & & $\begin{array}{c}\text { GalNAc4S- } \beta(1 \rightarrow 4)-\beta- \\
\text { GlcA }\end{array}$ & 0.822 & 62 & 121 & 300 \\
\hline & & $\begin{array}{c}\text { GalNAc6S- } \beta(1 \rightarrow 4)-\beta- \\
\text { GlcA }\end{array}$ & 1.027 & 70 & 138 & 310 \\
\hline & & $\begin{array}{c}\text { GalNAc4S- } \beta(1 \rightarrow 4)-\beta- \\
\text { GlcA2S }\end{array}$ & 0.714 & 45 & 94 & 254 \\
\hline & & $\begin{array}{c}\text { GalNAc6S- } \beta(1 \rightarrow 4)-\beta- \\
\text { GlcA2S }\end{array}$ & 0.837 & 57 & 118 & 327 \\
\hline & & $\begin{array}{c}\text { GalNAc4,6S- } \beta(1 \rightarrow 4)-\beta- \\
\text { GlcA }\end{array}$ & 1.042 & 74 & 182 & 370 \\
\hline & & $\begin{array}{c}\text { GalNAc4,6S- } \beta(1 \rightarrow 4)-\beta- \\
\text { GlcA2S }\end{array}$ & 0.841 & 53 & 135 & 394 \\
\hline \multirow{4}{*}{$\begin{array}{l}\text { Dermatan sulfate } \\
\text { (DS) }\end{array}$} & \multirow{4}{*}{$\begin{array}{l}\text { L-IdoA2R } R^{1}-\alpha(1 \rightarrow 3)- \\
\quad \beta-G a l N A c 4,6 R^{1}\end{array}$} & $\begin{array}{c}\text { L-IdoA- } \alpha(1 \rightarrow 3)-\beta- \\
\text { GalNAc }\end{array}$ & 0.296 & $149 \pm 3$ & $105 \pm 12$ & $441 \pm 11$ \\
\hline & & $\begin{array}{l}\text { L-IdoA- } \alpha(1 \rightarrow 3)-\beta- \\
\text { GalNAc4S }\end{array}$ & 0.814 & 71 & 50 & 287 \\
\hline & & $\begin{array}{l}\text { L-IdoA- } \alpha(1 \rightarrow 3)-\beta- \\
\text { GalNAc6S }\end{array}$ & 0.182 & 118 & 117 & 405 \\
\hline & & $\begin{array}{c}\text { L-IdoA2S- } \alpha(1 \rightarrow 3)-\beta- \\
\text { GalNAc }\end{array}$ & 0.865 & 126 & 153 & 683 \\
\hline
\end{tabular}




\begin{tabular}{|c|c|c|c|c|c|}
\hline & $\begin{array}{c}\text { L-IdoA2S- } \alpha(1 \rightarrow 3)-\beta- \\
\text { GalNAc4S }\end{array}$ & 0.747 & 113 & 52 & 407 \\
\hline & $\begin{array}{c}\text { L-IdoA2S- } \alpha(1 \rightarrow 3)-\beta- \\
\text { GalNAc6S }\end{array}$ & 0.839 & 135 & 94 & 597 \\
\hline & $\begin{array}{l}\text { L-IdoA- } \alpha(1 \rightarrow 3)-\beta- \\
\text { GalNAc4,6S }\end{array}$ & 0.806 & 63 & 54 & 285 \\
\hline & $\begin{array}{l}\text { L-IdoA2S- } \alpha(1 \rightarrow 3)-\beta- \\
\text { GalNAc4,6S }\end{array}$ & 0.755 & 53 & 54 & 243 \\
\hline \multirow{8}{*}{$\begin{array}{c}\text { GalNAc4,6R }{ }^{1-} \\
\beta(1 \rightarrow 4)-\alpha-\text { L-IdoA2R }^{1}\end{array}$} & $\begin{array}{c}\text { GalNAc- } \beta(1 \rightarrow 4)-\alpha-L- \\
\text { IdoA }\end{array}$ & 0.245 & $129 \pm 8$ & $230 \pm 14$ & $446 \pm 16$ \\
\hline & $\begin{array}{c}\text { GalNAc- } \beta(1 \rightarrow 4)-\alpha-L- \\
\text { IdoA2S }\end{array}$ & 0.375 & 146 & 269 & 483 \\
\hline & $\begin{array}{c}\text { GalNAc4S- } \beta(1 \rightarrow 4)-\alpha-L- \\
\text { IdoA }\end{array}$ & 0.246 & 116 & 214 & 438 \\
\hline & $\begin{array}{c}\text { GalNAc6S }-\beta(1 \rightarrow 4)-\alpha-L- \\
\text { IdoA }\end{array}$ & 0.337 & 123 & 231 & 437 \\
\hline & $\begin{array}{c}\text { GalNAc4S- } \beta(1 \rightarrow 4)-\alpha-L- \\
\text { IdoA2S }\end{array}$ & 0.303 & 106 & 229 & 439 \\
\hline & $\begin{array}{c}\text { GalNAc6S- } \beta(1 \rightarrow 4)-\alpha-L- \\
\text { IdoA2S }\end{array}$ & 0.393 & 100 & 198 & 403 \\
\hline & $\begin{array}{c}\text { GalNAc4,6S- } \beta(1 \rightarrow 4)-\alpha- \\
\text { L-IdoA }\end{array}$ & 0.310 & 116 & 215 & 421 \\
\hline & $\begin{array}{c}\text { GalNAc4,6S- } \beta(1 \rightarrow 4)-\alpha- \\
\text { L-IdoA2S }\end{array}$ & 0.337 & 78 & 158 & 323 \\
\hline
\end{tabular}




\begin{tabular}{|c|c|c|c|c|c|c|}
\hline \multirow{8}{*}{$\begin{array}{l}\text { Keratan sulfate } \\
\quad(\mathrm{KS})\end{array}$} & \multirow{4}{*}{$\begin{array}{l}\text { Gal6R }{ }^{1}-\beta(1 \rightarrow 4)-\beta- \\
\quad \text { GlcNAc6R }^{1}\end{array}$} & Gal- $\beta(1 \rightarrow 4)-\beta-G \mid c N A c$ & 0.441 & $49 \pm 6$ & $103 \pm 3$ & $363 \pm 20$ \\
\hline & & Gal- $\beta(1 \rightarrow 4)-\beta-$ GlcNAc6S & 0.474 & 50 & 107 & 305 \\
\hline & & Gal6S- $\beta(1 \rightarrow 4)-\beta-$ GlcNAc & 0.650 & 43 & 91 & 312 \\
\hline & & $\begin{array}{l}\text { Gal6S- } \beta(1 \rightarrow 4)-\beta- \\
\text { GlcNAc6S }\end{array}$ & 0.741 & 43 & 93 & 291 \\
\hline & \multirow{4}{*}{$\begin{array}{c}\text { GlcNAc6R }{ }^{1-} \\
\beta(1 \rightarrow 3)-\beta-\text { Gal6R}^{1}\end{array}$} & GlcNAc- $\beta(1 \rightarrow 3)-\beta-$ Gal & 0.079 & $200 \pm 2$ & $338 \pm 4$ & $571 \pm 12$ \\
\hline & & GlcNAc- $\beta(1 \rightarrow 3)-\beta-$ Gal6S & 0.120 & 143 & 285 & 514 \\
\hline & & GlcNAc6S- $\beta(1 \rightarrow 3)-\beta-G a l$ & 0.407 & 86 & 249 & 511 \\
\hline & & $\begin{array}{c}\text { GlcNAc6S- } \beta(1 \rightarrow 3)-\beta- \\
\text { Gal6S }\end{array}$ & 0.194 & 85 & 260 & 556 \\
\hline \multirow{4}{*}{$\begin{array}{c}\text { Heparin/Heparan } \\
\text { sulfate (HP/HS) }\end{array}$} & \multirow{4}{*}{$\begin{array}{l}\text { L-IdoA2R } R^{1}-\alpha(1 \rightarrow 4)- \\
\alpha-G I^{2} R^{2} 3,6 R^{1}\end{array}$} & L-IdoA- $\alpha(1 \rightarrow 4)-\alpha-$ GlcNAc & 0.437 & $123 \pm 10$ & $240 \pm 8$ & $611 \pm 36$ \\
\hline & & $\begin{array}{c}\text { L-IdoA- } \alpha(1 \rightarrow 4)-\alpha- \\
\text { GlcNAc3S }\end{array}$ & 1.205 & 35 & 106 & 271 \\
\hline & & $\begin{array}{l}\text { L-IdoA- } \alpha(1 \rightarrow 4)-\alpha- \\
\text { GlcNAc6S }\end{array}$ & 0.447 & 35 & 127 & 326 \\
\hline & & $\begin{array}{c}\text { L-IdoA2S- }-\alpha(1 \rightarrow 4)-\alpha- \\
\text { GIcNAc }\end{array}$ & 1.033 & 73 & 153 & 308 \\
\hline
\end{tabular}




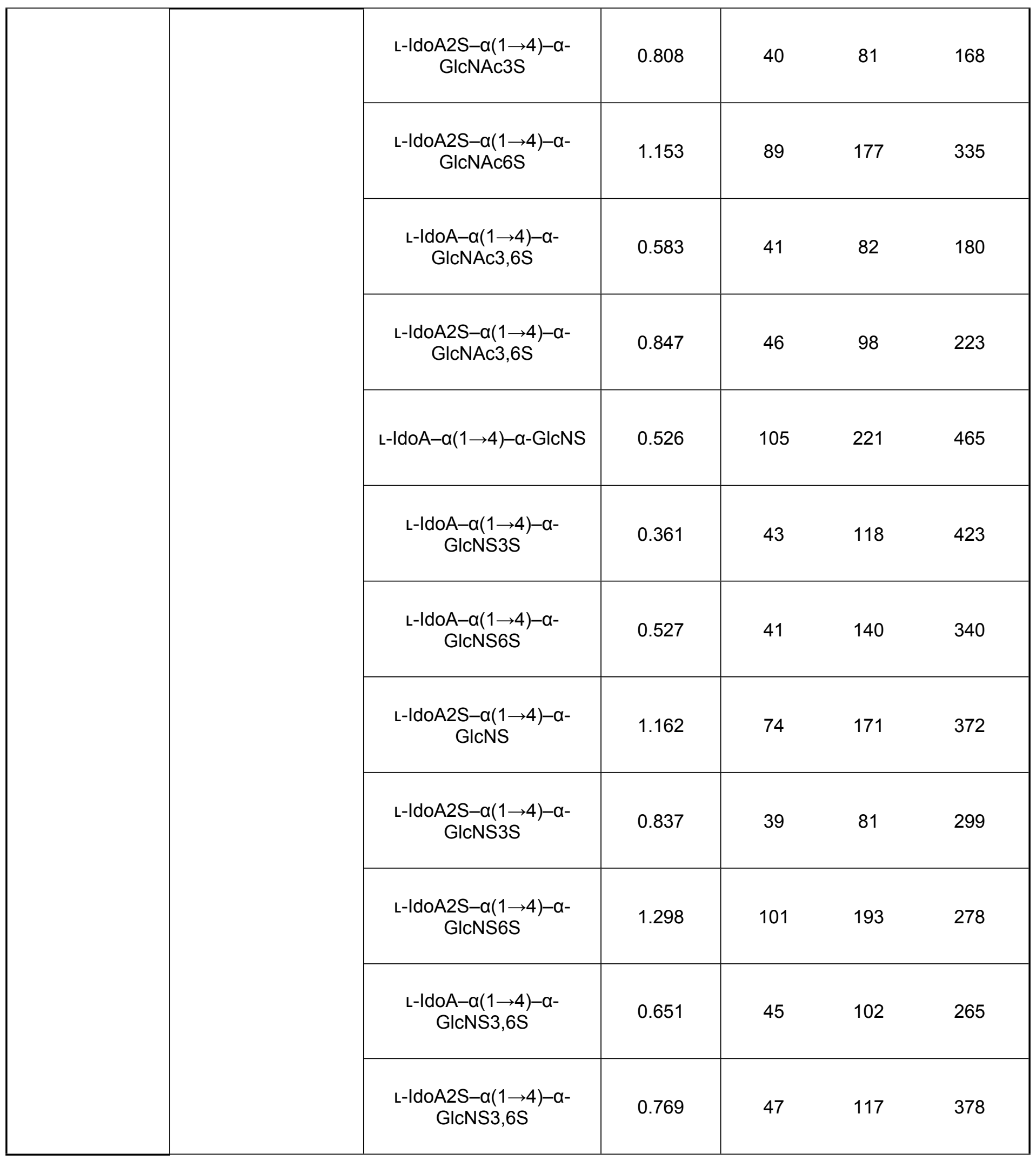




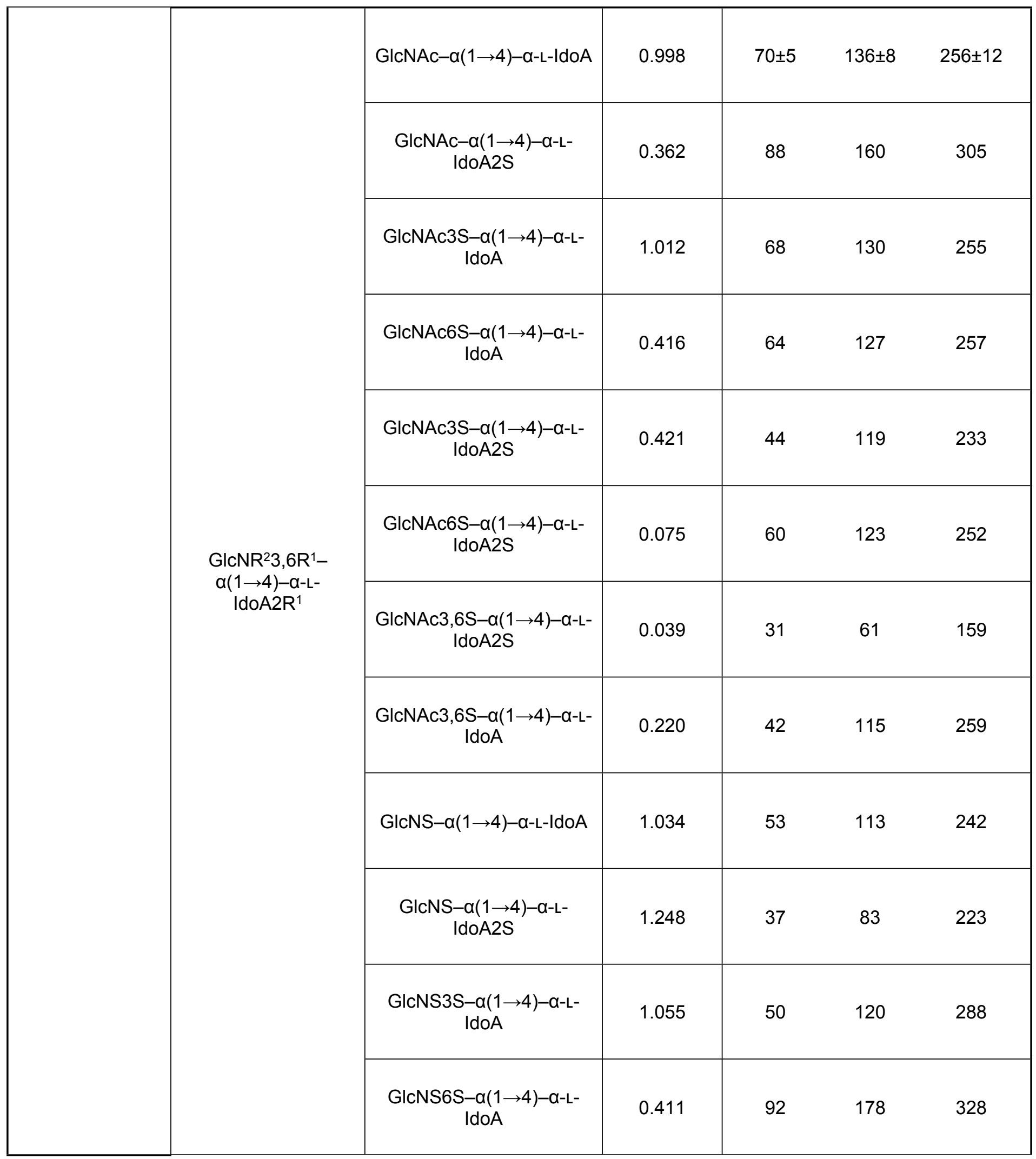




\begin{tabular}{|c|c|c|c|c|c|}
\hline & $\begin{array}{c}\text { GlcNS3S- } \alpha(1 \rightarrow 4)-\alpha-L- \\
\text { IdoA2S }\end{array}$ & 0.472 & 42 & 87 & 189 \\
\hline & $\begin{array}{c}\text { GlcNS6S- } \alpha(1 \rightarrow 4)-\alpha-L- \\
\text { IdoA2S }\end{array}$ & 0.670 & 53 & 141 & 321 \\
\hline & $\begin{array}{c}\text { GlcNS3,6S- } \alpha(1 \rightarrow 4)-\alpha-L- \\
\text { IdoA }\end{array}$ & 0.229 & 92 & 206 & 418 \\
\hline & $\begin{array}{c}\text { GlcNS3,6S- } \alpha(1 \rightarrow 4)-\alpha-L- \\
\text { IdoA2S }\end{array}$ & 0.257 & 45 & 158 & 368 \\
\hline \multirow{8}{*}{$\begin{array}{c}\text { GlcA2R }{ }^{1}-\beta(1 \rightarrow 4)-\alpha- \\
\text { GlcNR }^{2} 3,6 R^{1}\end{array}$} & GlcA- $\beta(1 \rightarrow 4)-\alpha-G \mid c N A c$ & 0.451 & $52 \pm 3$ & $111 \pm 17$ & $348 \pm 39$ \\
\hline & $\begin{array}{l}\text { GlcA- } \beta(1 \rightarrow 4)-\alpha- \\
\text { GlcNAc3S }\end{array}$ & 0.052 & 32 & 65 & 224 \\
\hline & $\begin{array}{l}\text { GlcA- } \beta(1 \rightarrow 4)-\alpha- \\
\text { GlcNAc6S }\end{array}$ & 0.489 & 50 & 106 & 254 \\
\hline & $\begin{array}{c}\text { GlcA2S- } \beta(1 \rightarrow 4)-\alpha- \\
\text { GIcNAc }\end{array}$ & 0.605 & 50 & 118 & 257 \\
\hline & $\begin{array}{c}\text { GlcA2S- } \beta(1 \rightarrow 4)-\alpha- \\
\text { GlcNAc3S }\end{array}$ & 0.076 & 24 & 51 & 115 \\
\hline & $\begin{array}{l}\text { GlcA2S- } \beta(1 \rightarrow 4)-\alpha- \\
\text { GlcNAc6S }\end{array}$ & 0.485 & 54 & 109 & 230 \\
\hline & $\begin{array}{l}\text { GlcA- } \beta(1 \rightarrow 4)-\alpha- \\
\text { GlcNAc3,6S }\end{array}$ & 0.070 & 32 & 65 & 139 \\
\hline & $\begin{array}{l}\text { GlcA2S- } \beta(1 \rightarrow 4)-\alpha- \\
\text { GlcNAc3,6S }\end{array}$ & 0.050 & 25 & 52 & 113 \\
\hline
\end{tabular}




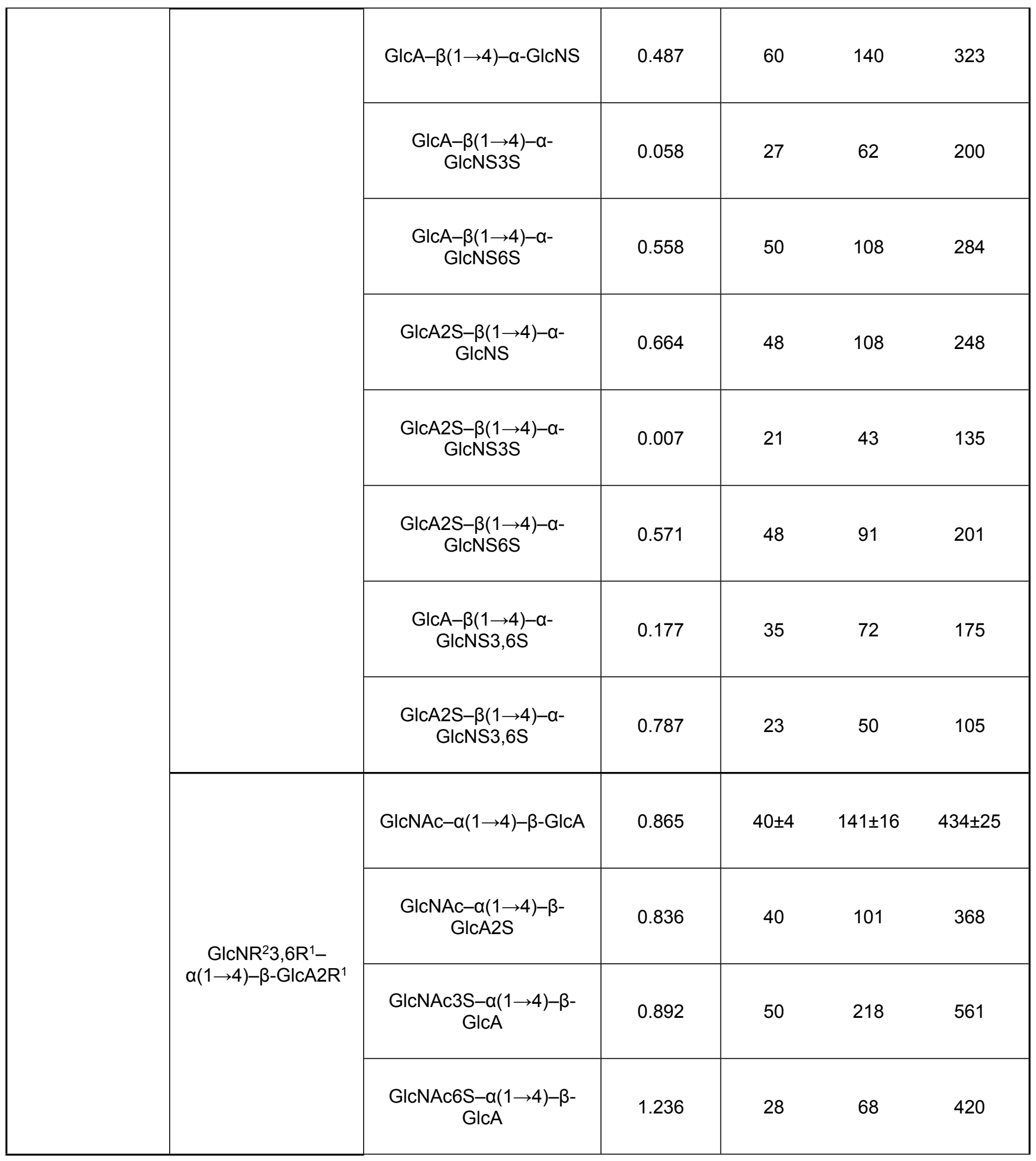




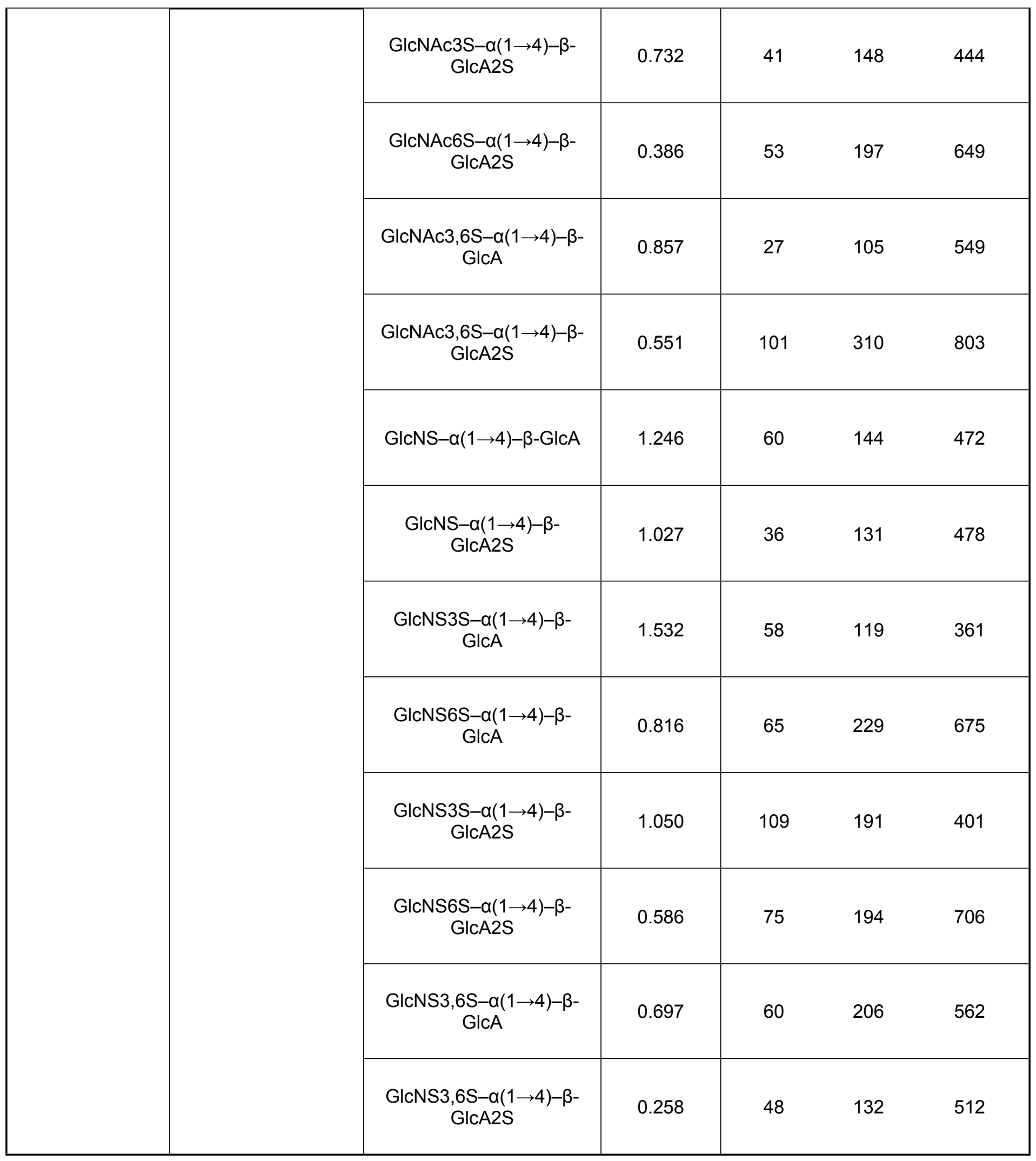




\section{Error analysis (Fig. S2)}

\section{Hyaluronic acid (HA)}

a. GlcA- $\beta(1 \rightarrow 3)-\beta-G \mid c N A c$

Free energy maps from 4 independent metadynamics simulations (10 ns):
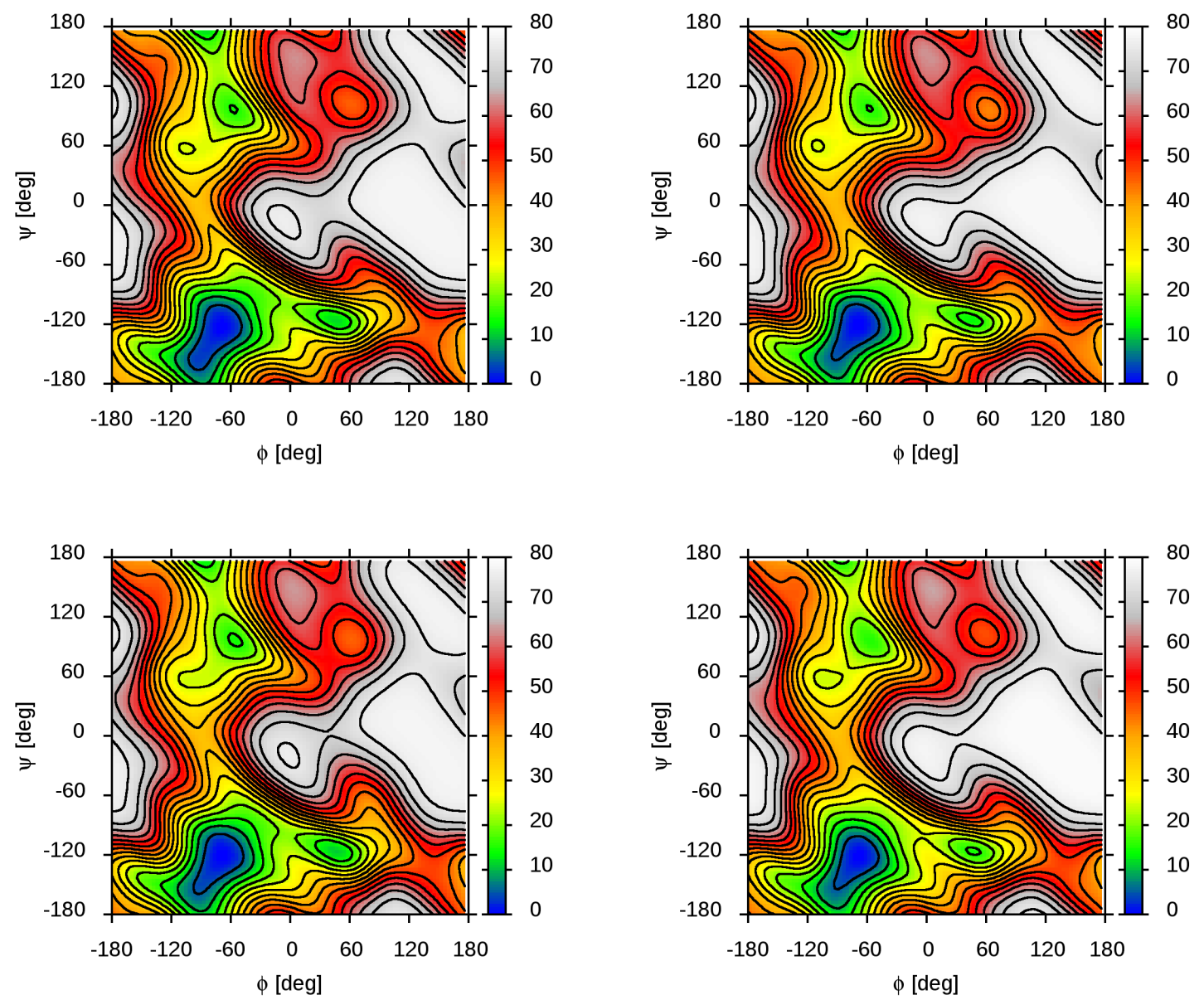

Free energy map from elongated metadynamics simulation (30 ns):

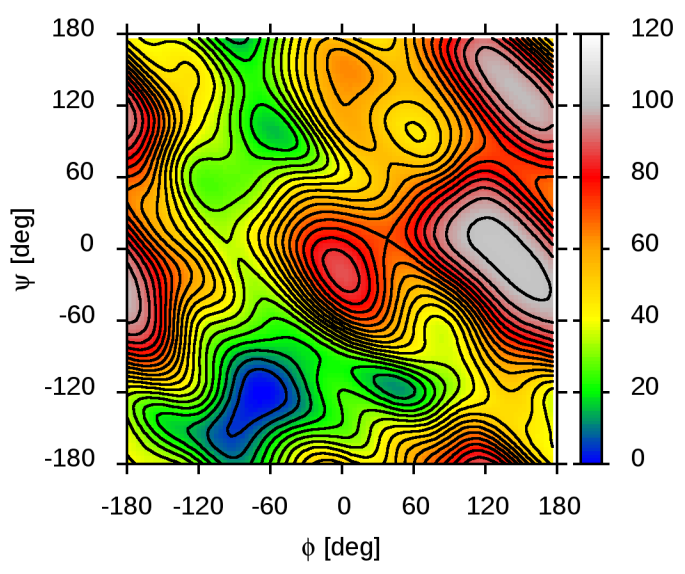


b. GlcNAc- $\beta(1 \rightarrow 4)-\beta-G \mid c A$

Free energy maps from 4 independent metadynamics simulations (10 ns):
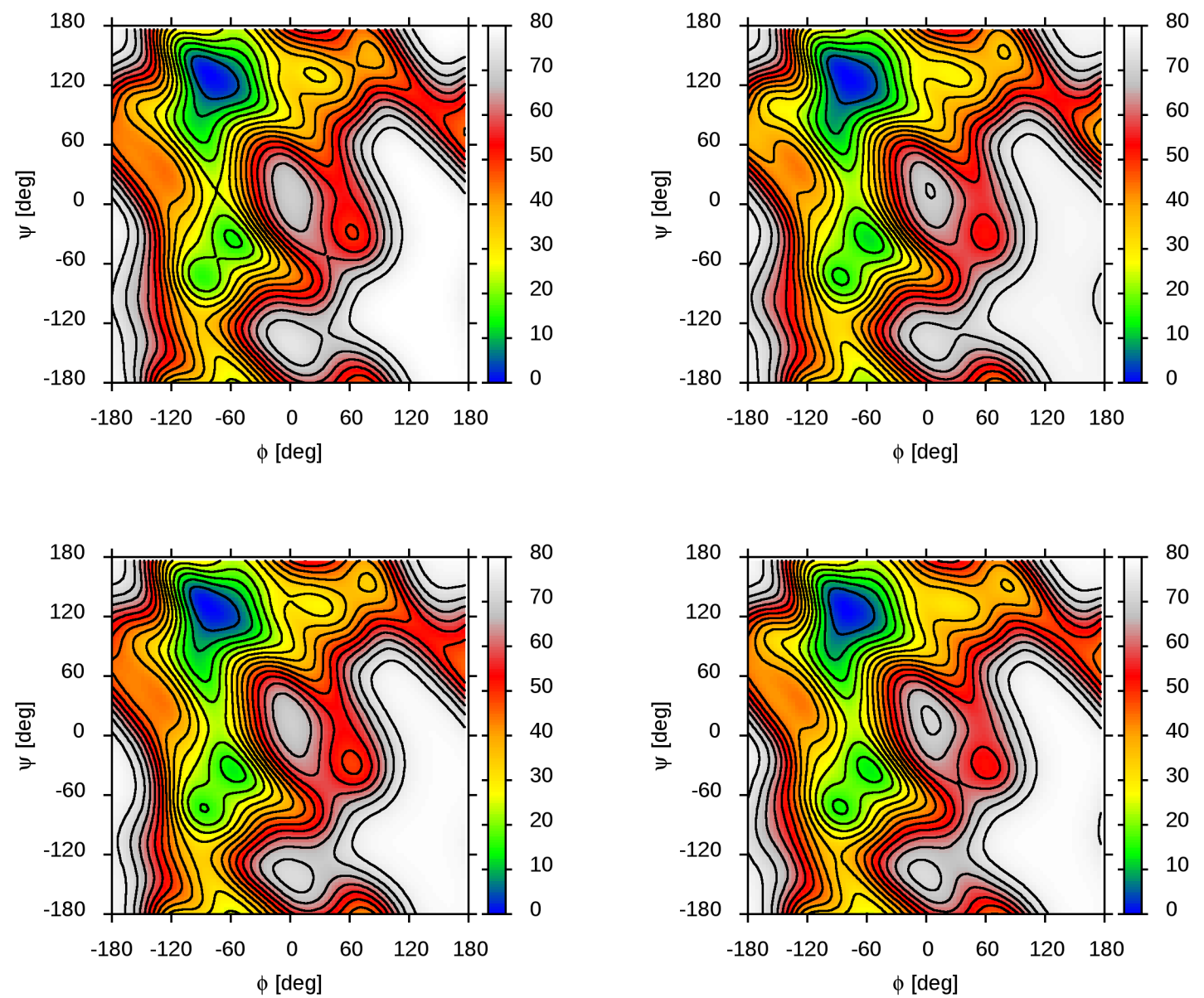

Free energy map from elongated metadynamics simulation (30 ns):

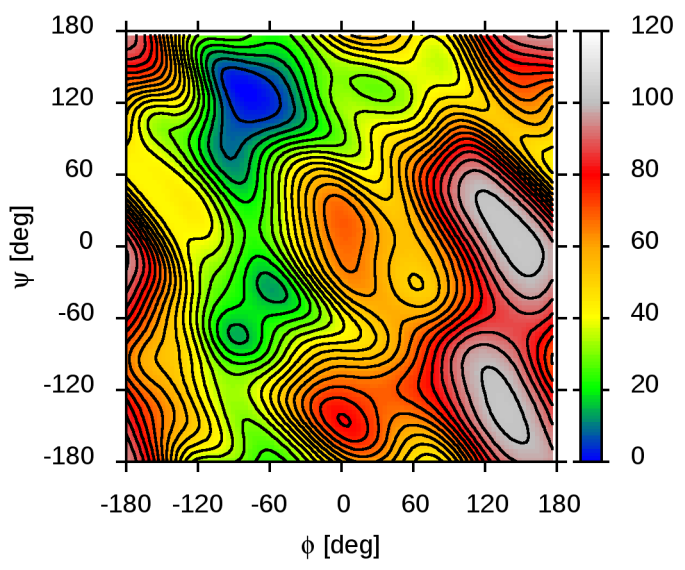




\section{Chondroitin sulfate (CS)}

a. GlcA- $\beta(1 \rightarrow 3)-\beta$-GalNAc

Free energy maps from 4 independent metadynamics simulations (10 ns):
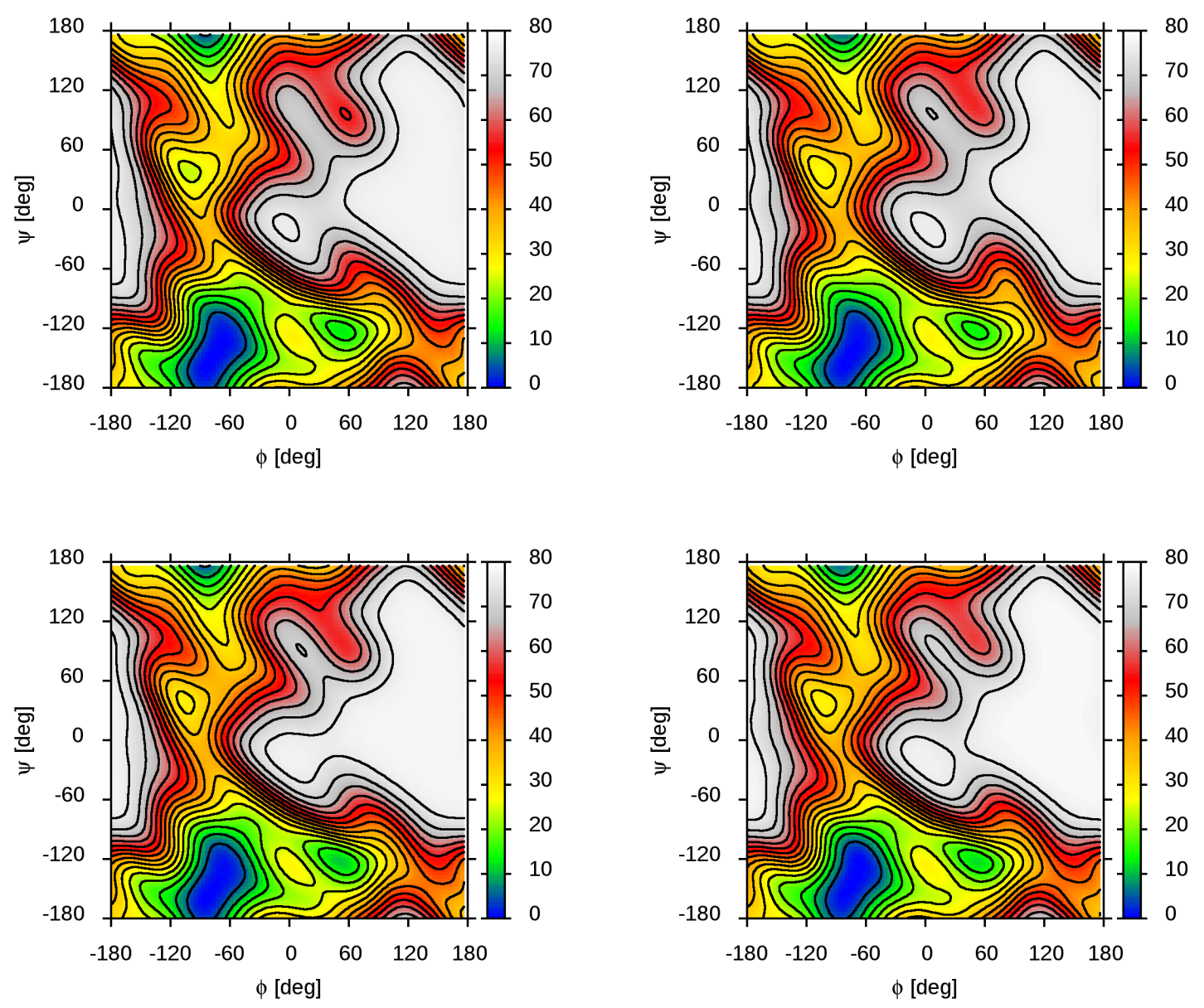

Free energy map from elongated metadynamics simulation (30 ns):

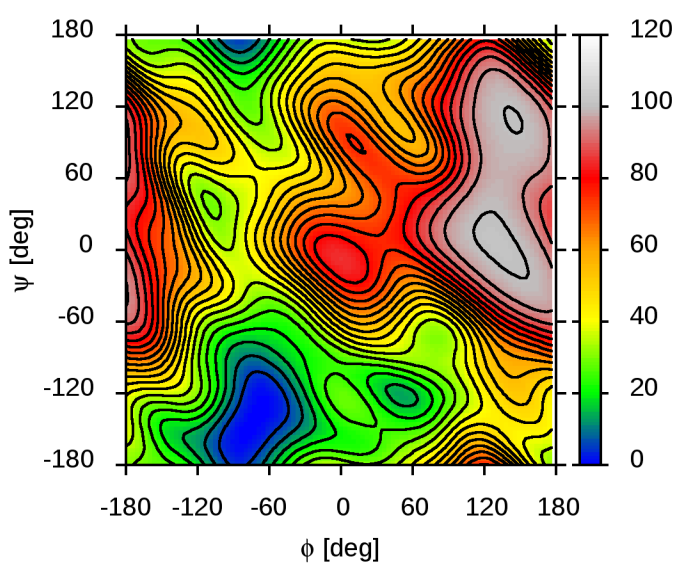


b. GalNAc- $\beta(1 \rightarrow 4)-\beta-G \mid c A$

Free energy maps from 4 independent metadynamics simulations (10 ns):
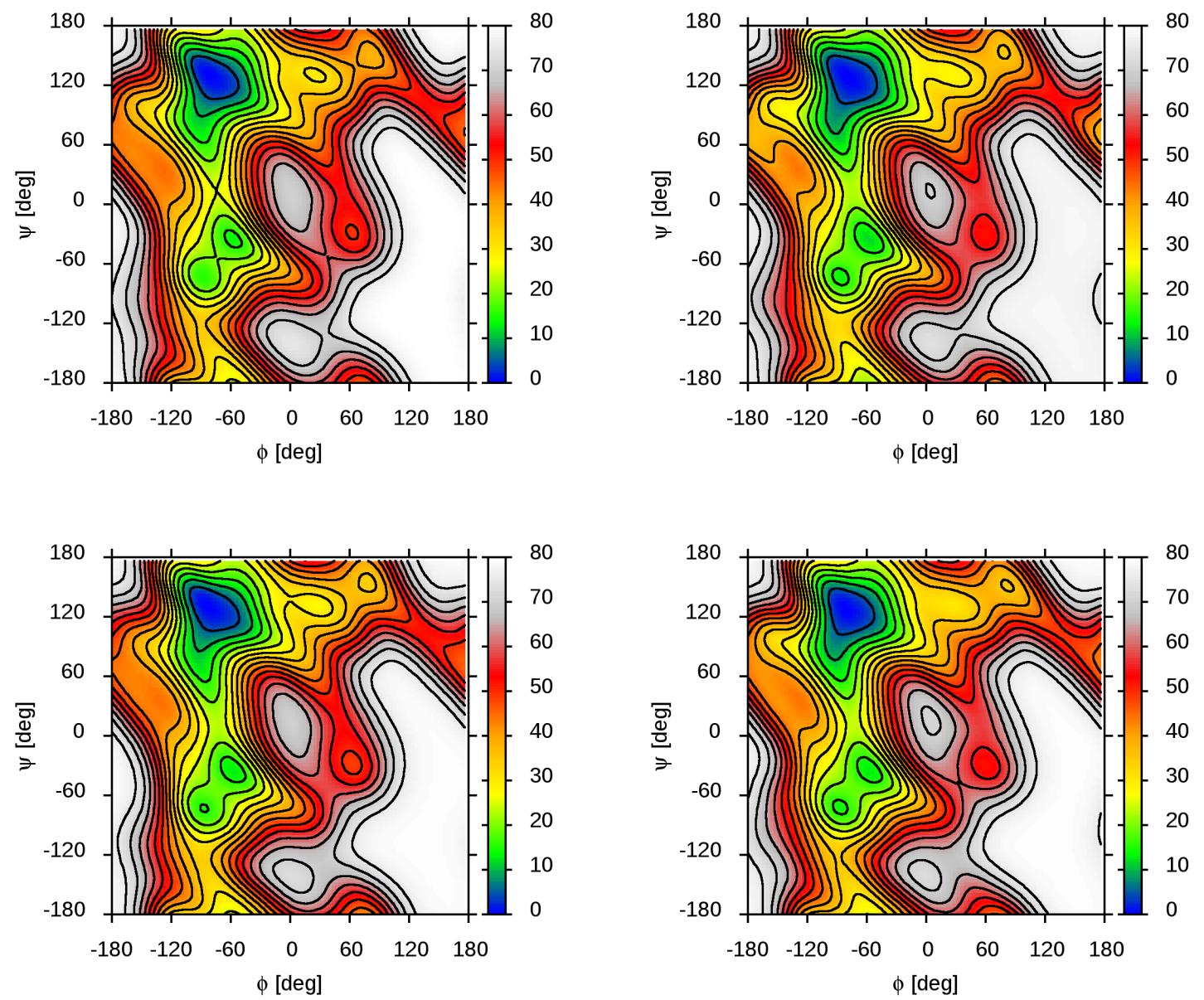

Free energy map from elongated metadynamics simulation (30 ns):

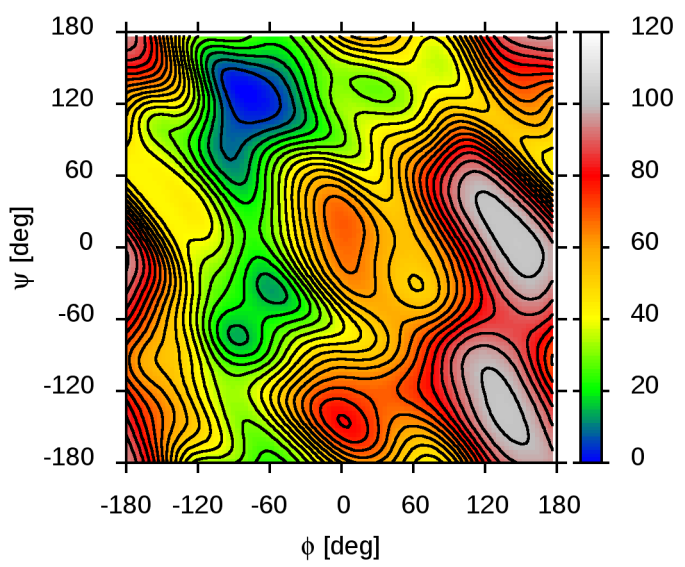




\section{Dermatan sulfate (DS)}

a. L-IdoA- $\alpha(1 \rightarrow 3)-\beta-$ GalNAc

Free energy maps from 4 independent metadynamics simulations (10 ns):
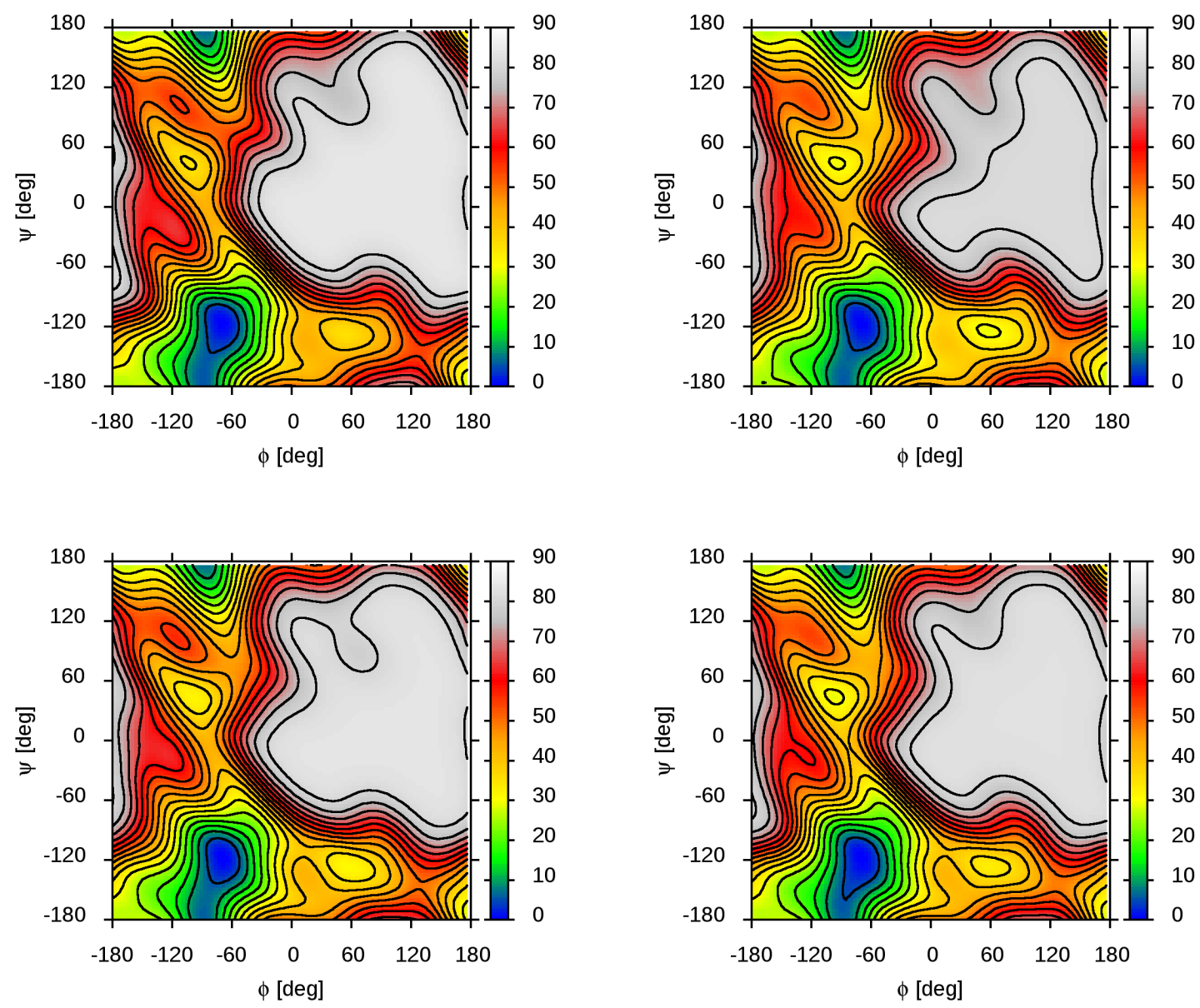

Free energy map from elongated metadynamics simulation (30 ns):

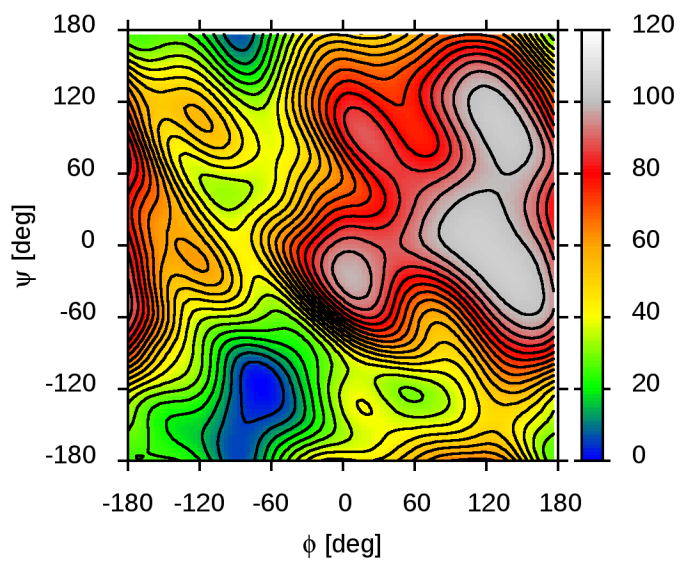


b. GalNAc- $\beta(1 \rightarrow 4)-\alpha-L-I d o A$

Free energy maps from 4 independent metadynamics simulations (10 ns):
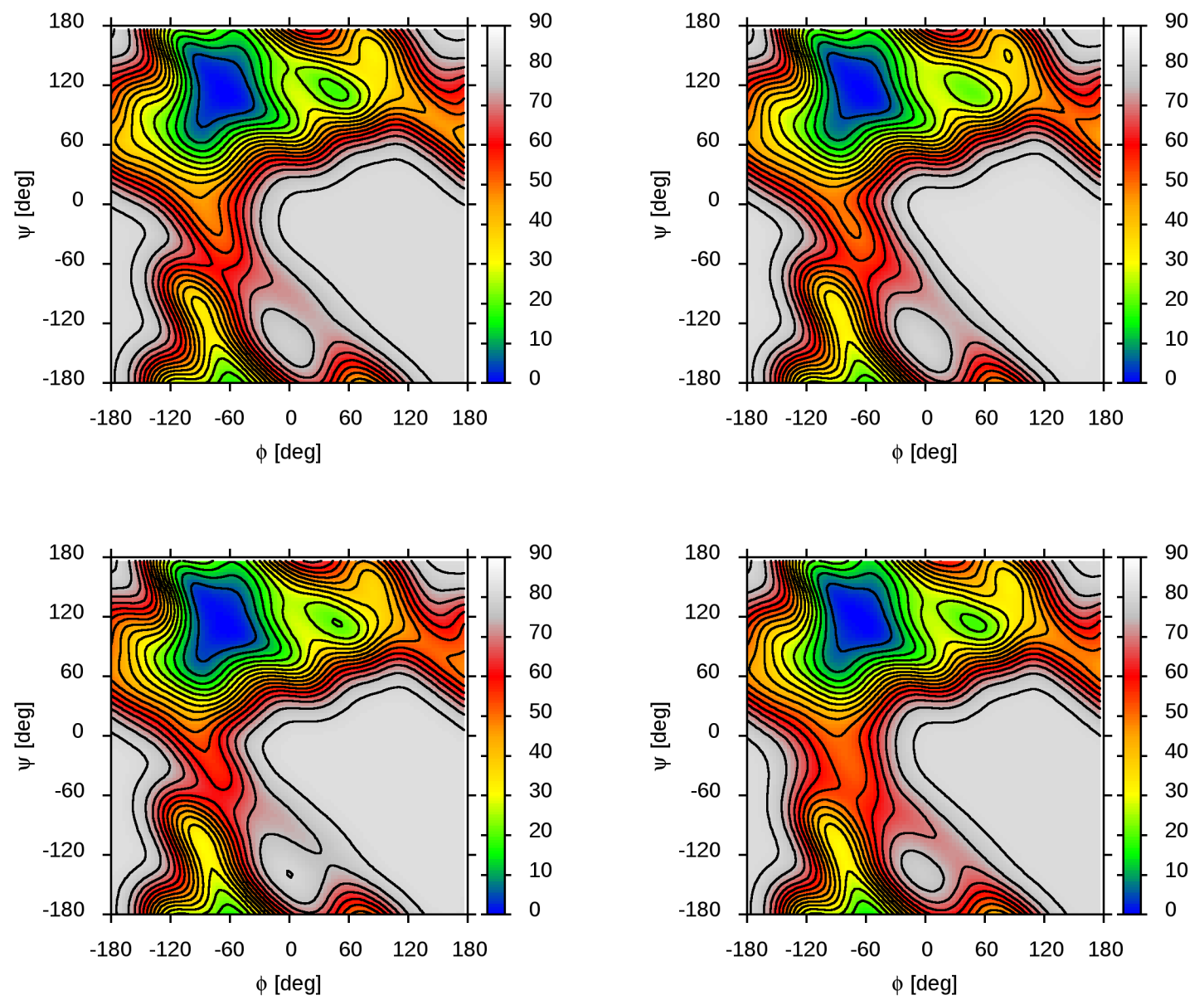

Free energy map from elongated metadynamics simulation (30 ns):

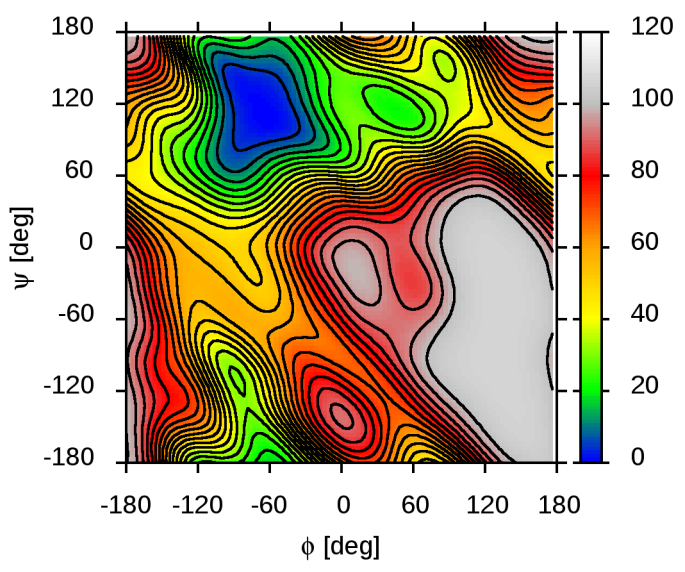




\section{Keratan sulfate (KS)}

a. Gal- $\beta(1 \rightarrow 4)-\beta-$ GlcNAc

Free energy maps from 4 independent metadynamics simulations (10 ns):
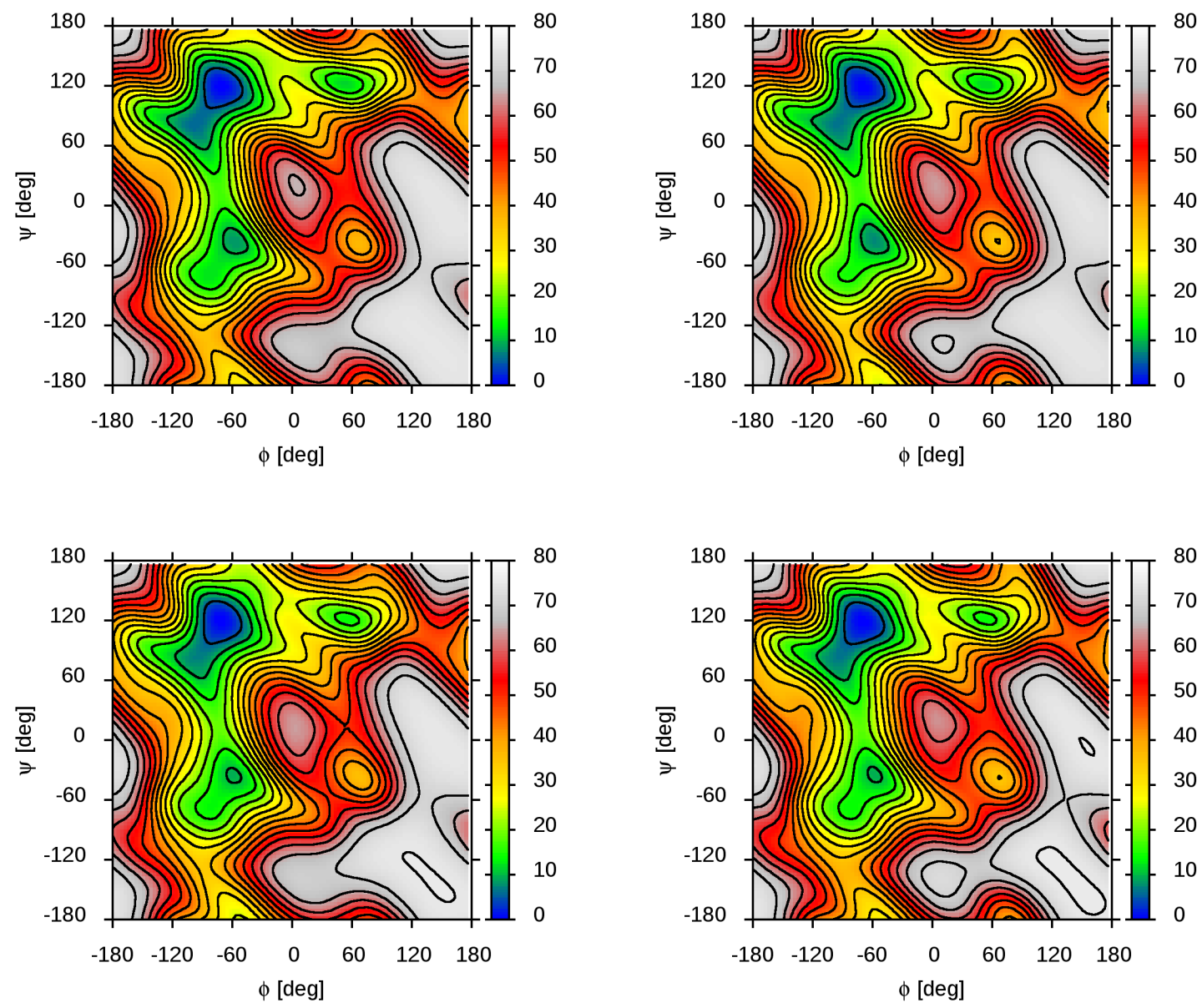

Free energy map from elongated metadynamics simulation (30 ns):

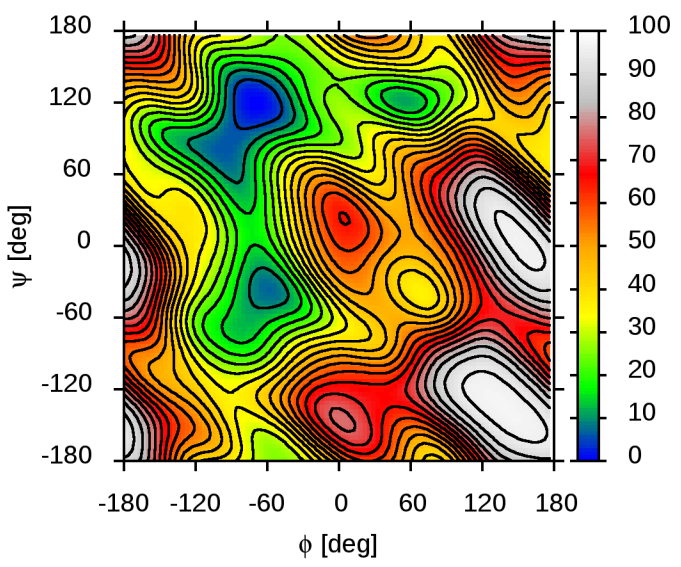


b. GlcNAc- $\beta(1 \rightarrow 3)-\beta-$ Gal

Free energy maps from 4 independent metadynamics simulations (10 ns):
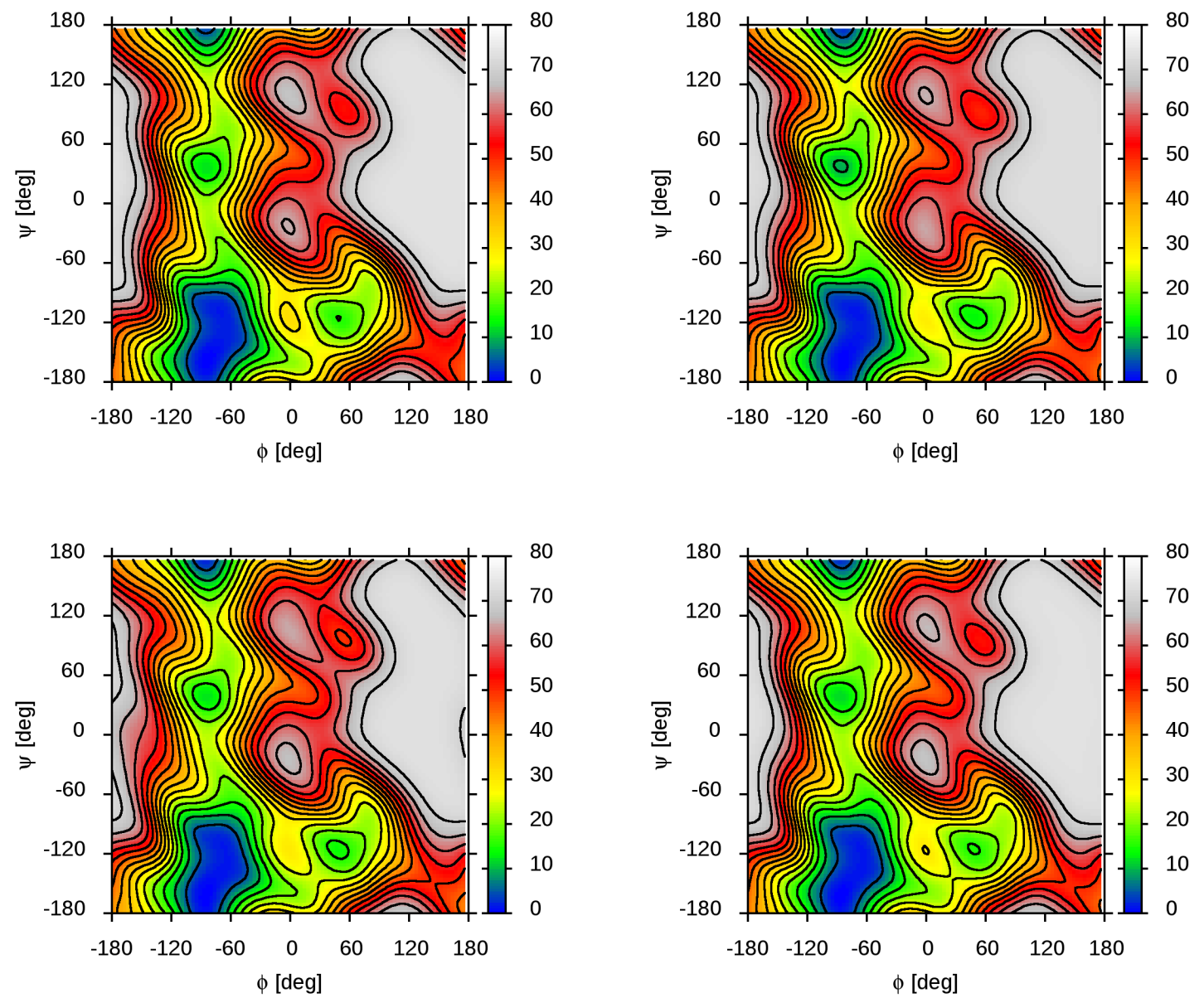

Free energy map from elongated metadynamics simulation (30 ns):

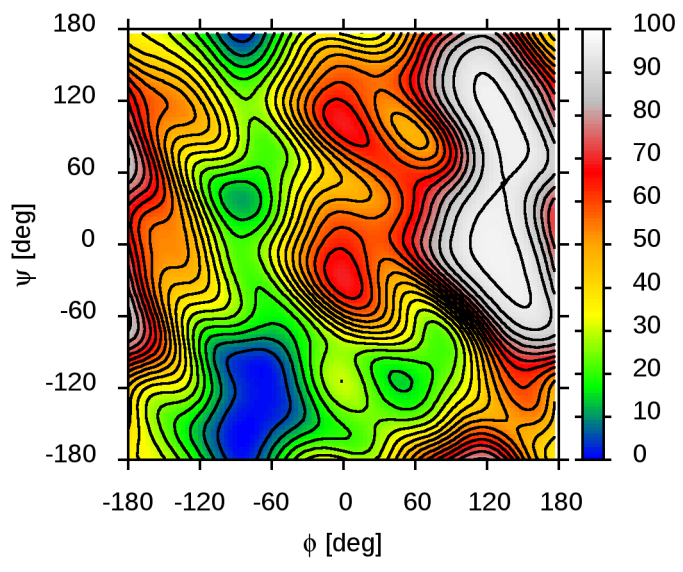




\section{Heparin/Heparan sulfate (HP/HS)}

a. L-IdoA- $\alpha(1 \rightarrow 4)-\alpha-G \mid c N A c$

Free energy maps from 4 independent metadynamics simulations (10 ns):
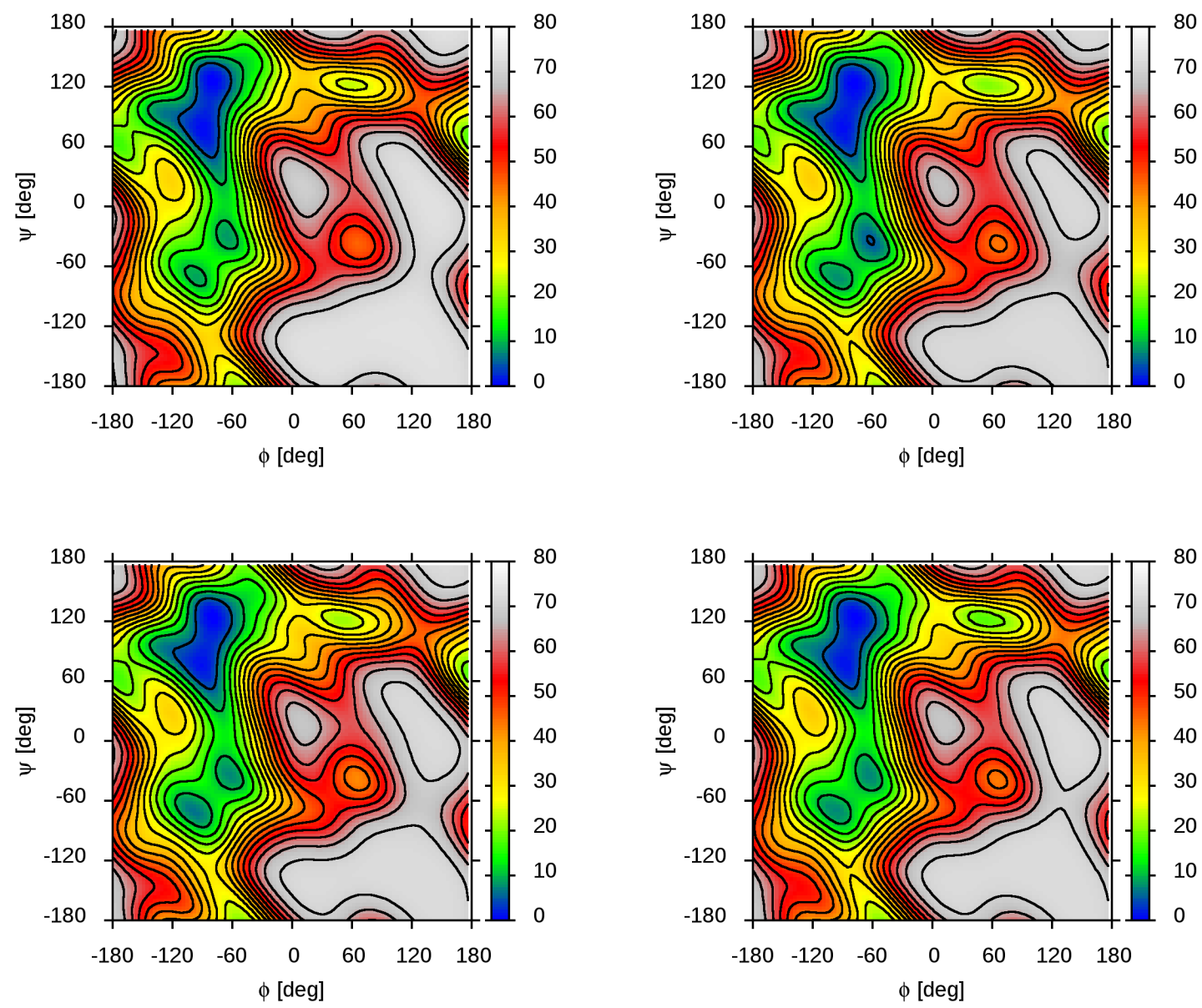

Free energy map from elongated metadynamics simulation (30 ns):

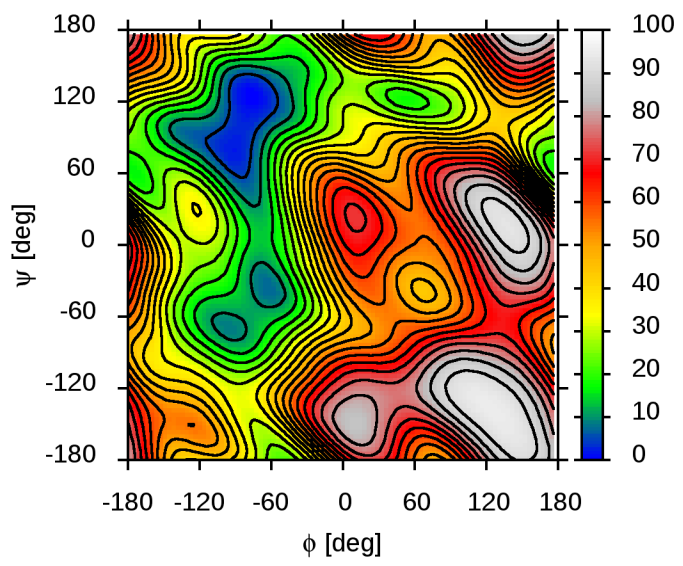


b. GlcNAc- $\alpha(1 \rightarrow 4)-\alpha-L-I d o A$

Free energy maps from 4 independent metadynamics simulations (10 ns):
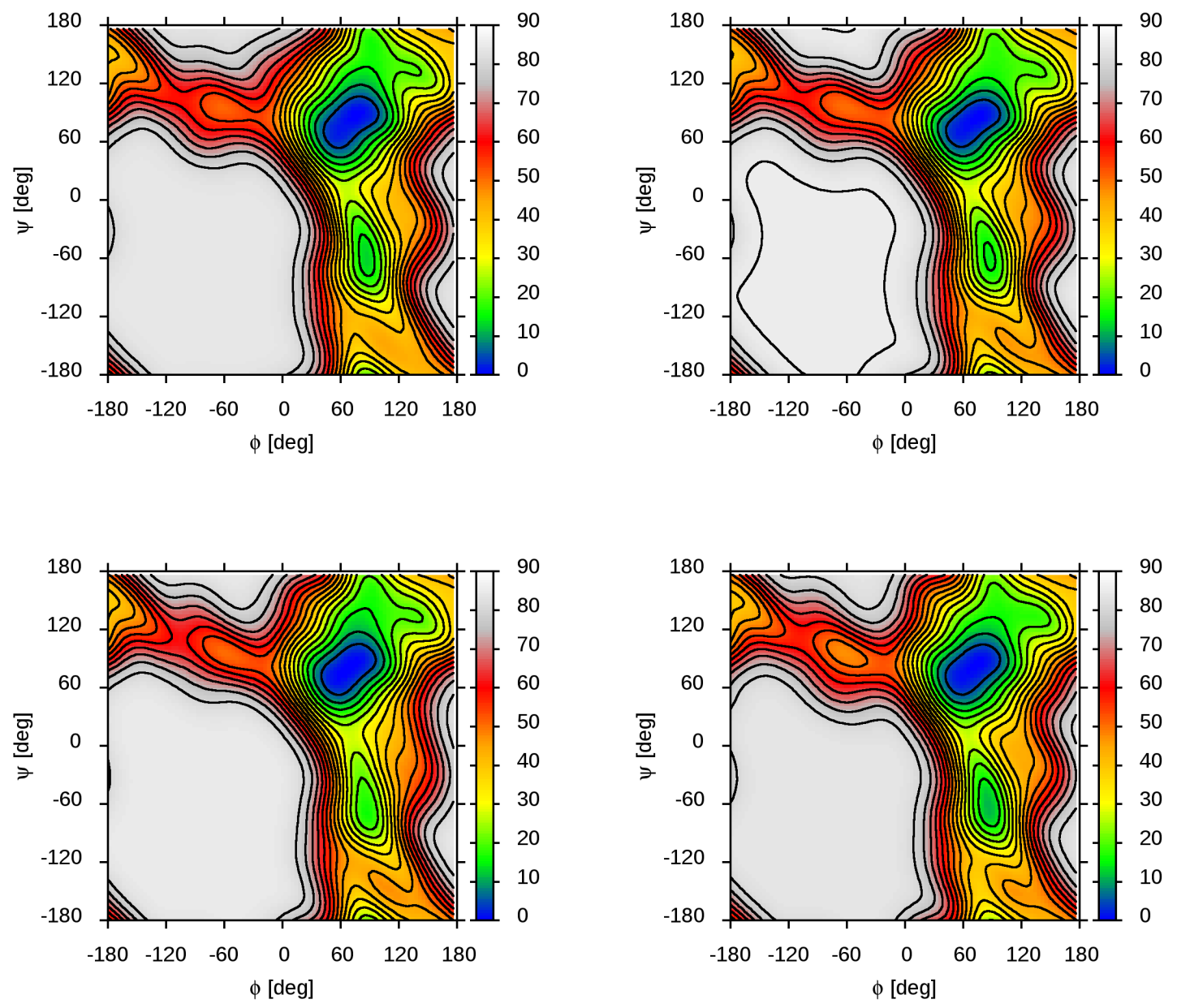

Free energy map from elongated metadynamics simulation (30 ns):

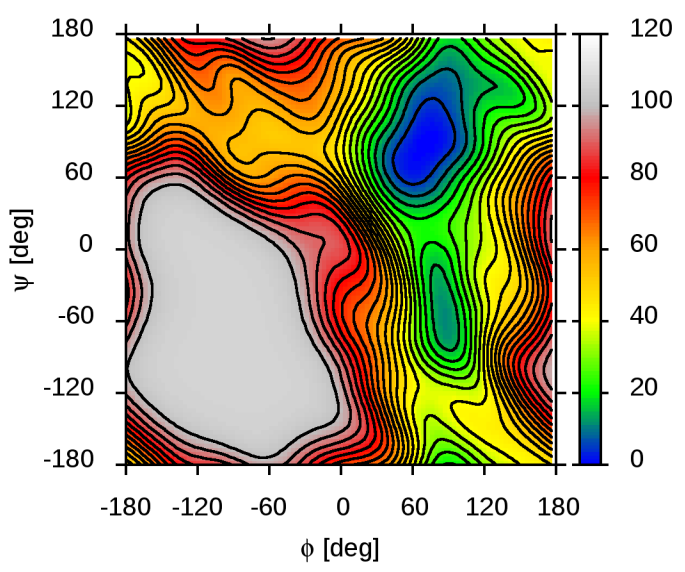




\section{c. GlcA- $\beta(1 \rightarrow 4)-\alpha-G \mid c N A c$}

Free energy maps from 4 independent metadynamics simulations (10 ns):
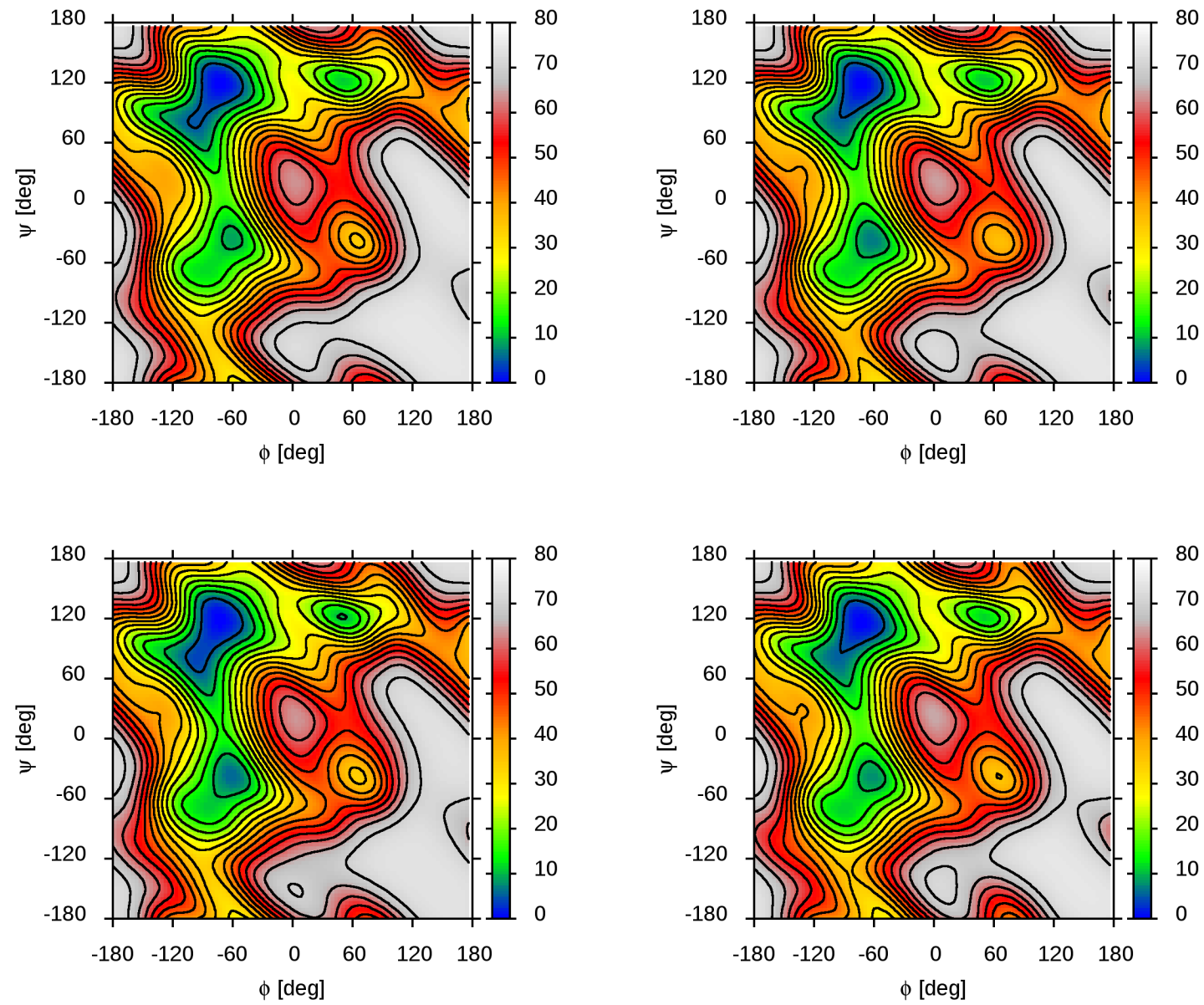

Free energy map from elongated metadynamics simulation (30 ns):

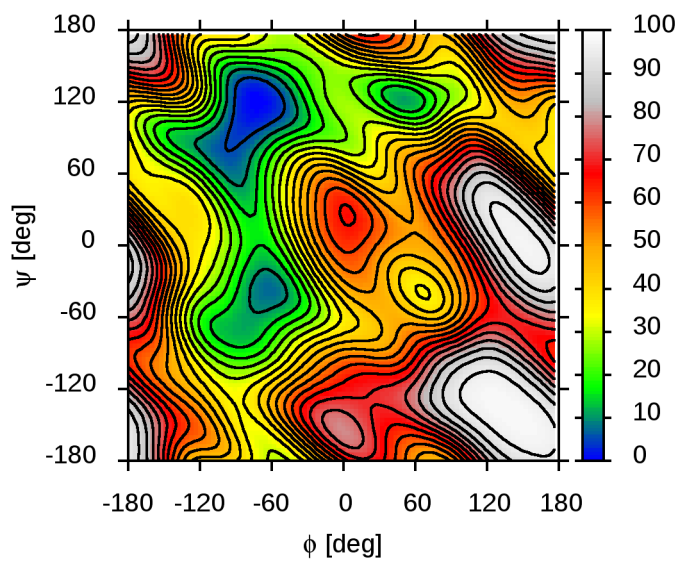


d. GlcNAc- $\alpha(1 \rightarrow 4)-\beta-G \mid c A$

Free energy maps from 4 independent metadynamics simulations (10 ns):
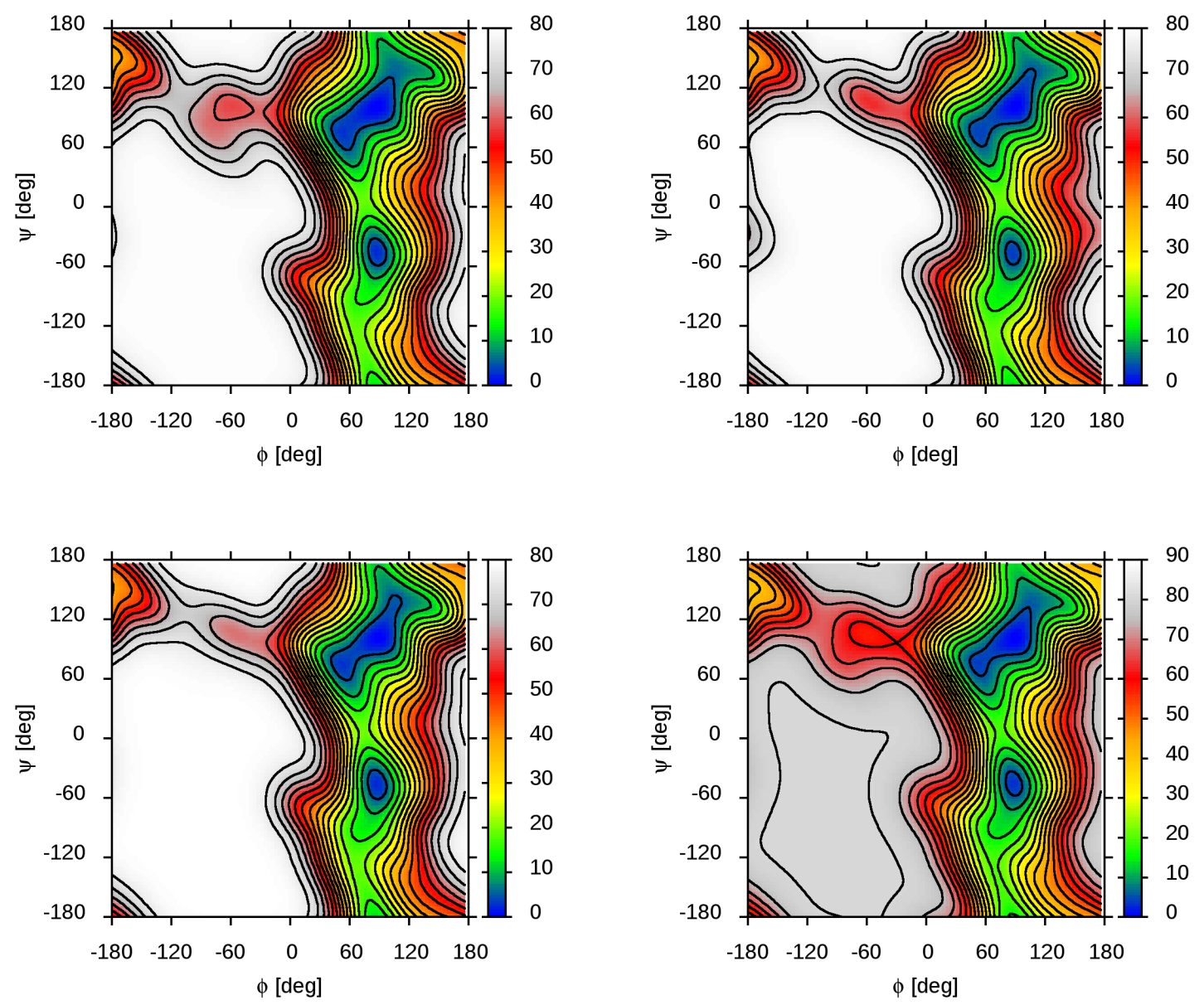

Free energy map from elongated metadynamics simulation (30 ns):

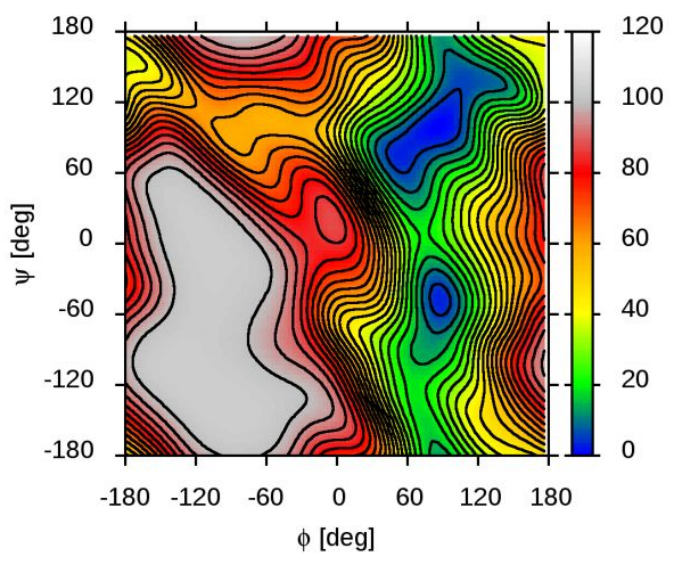


Analysis of standard deviations inherent to independently calculated FEMs (Fig. S3)

I. Hyaluronic acid (HA)

a. GlcA- $\beta(1 \rightarrow 3)-\beta-$ GlcNAc

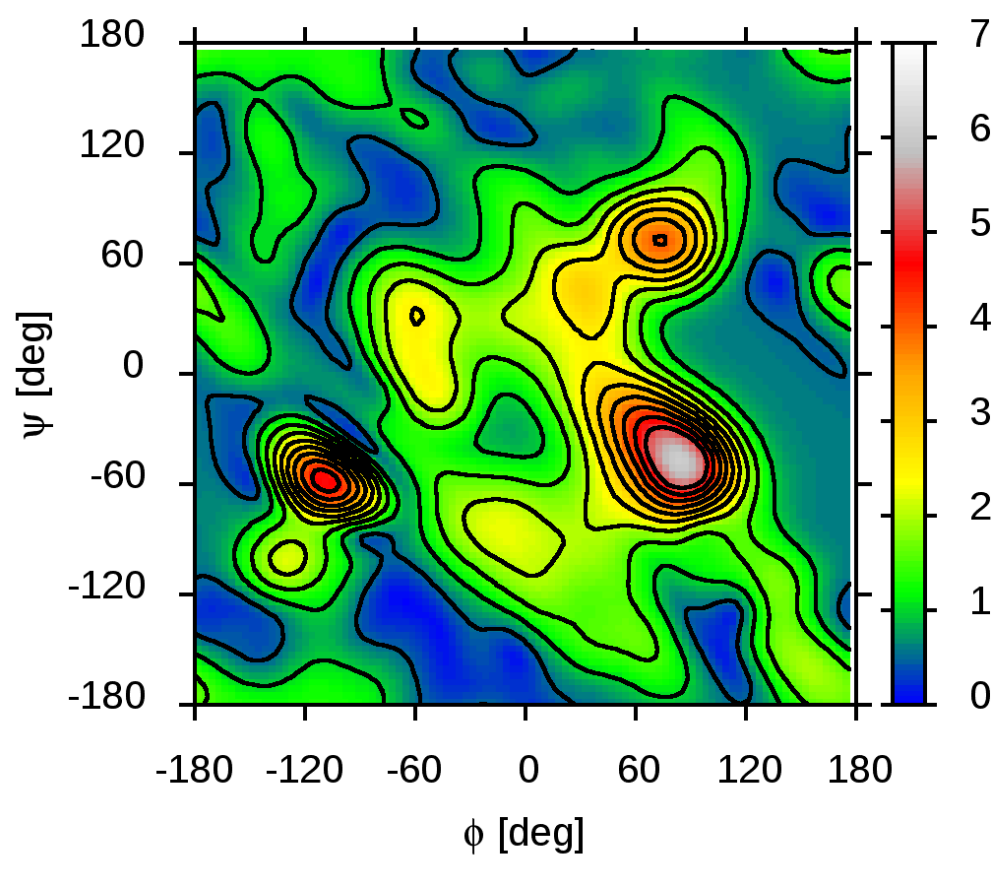


b. GlcNAc- $\beta(1 \rightarrow 4)-\beta-G l c A$

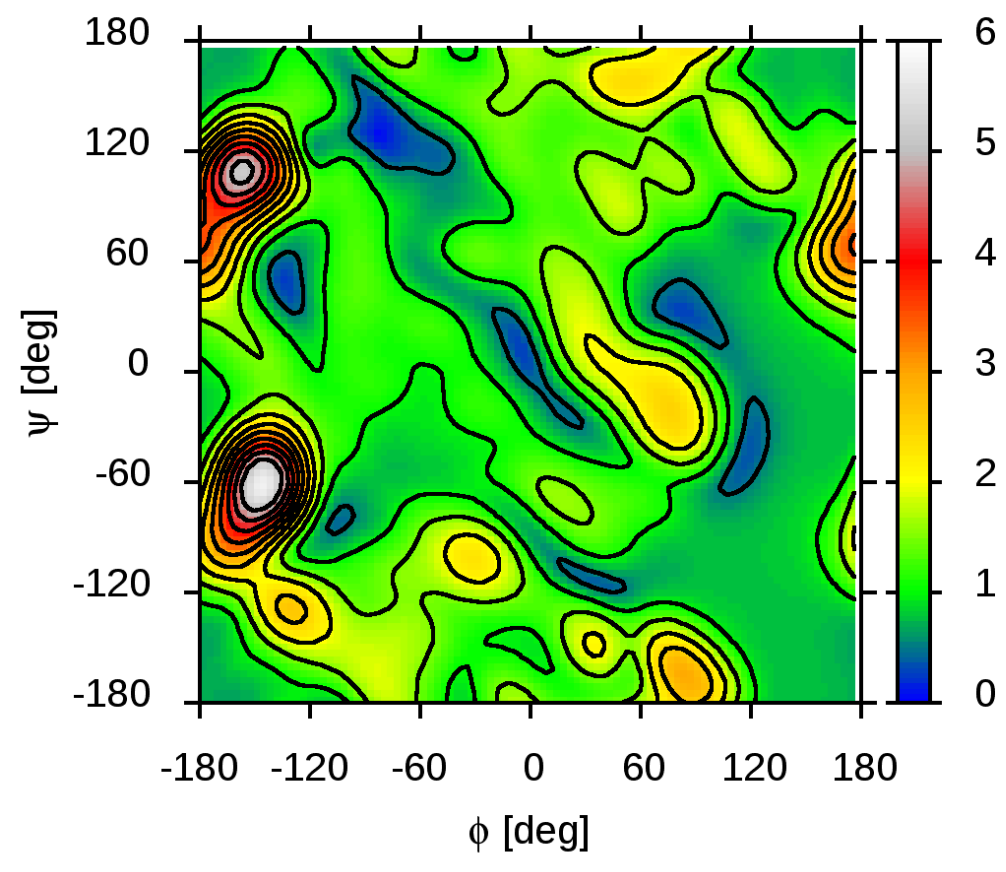




\section{Chondroitin sulfate (CS)}

a. GlcA- $\beta(1 \rightarrow 3)-\beta-$ GalNAc

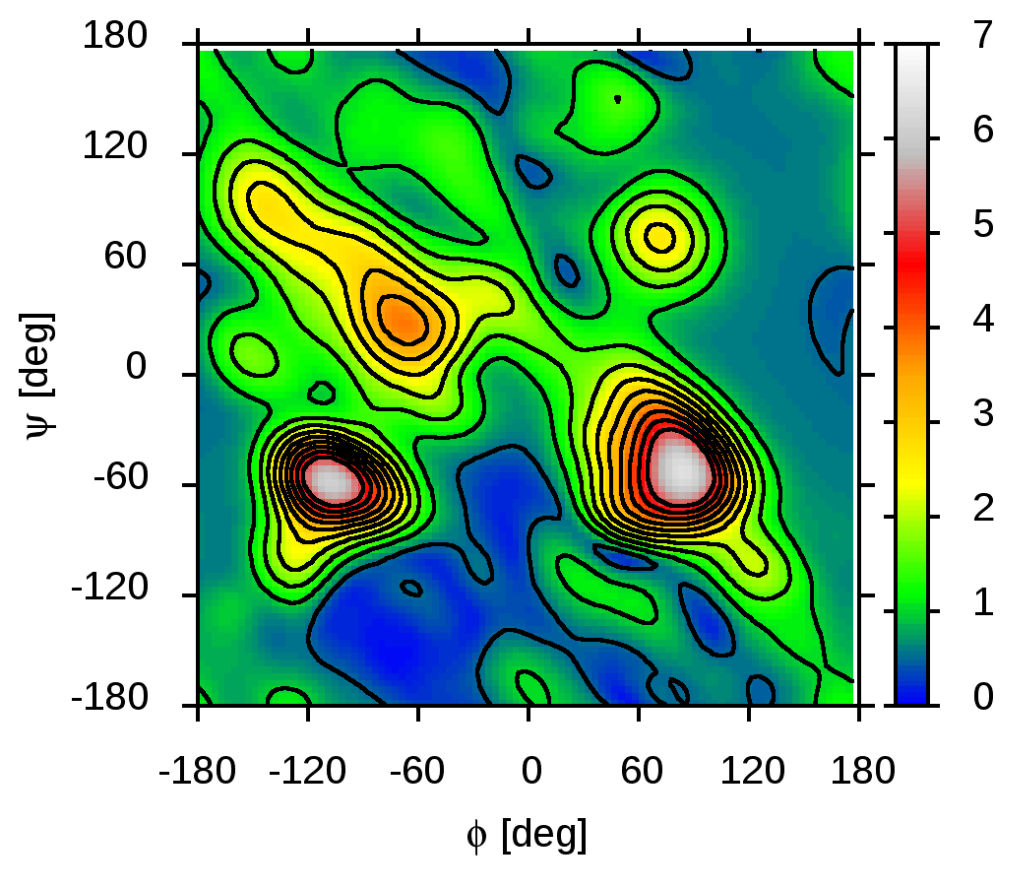

b. GalNAc- $\beta(1 \rightarrow 4)-\beta-G \mid c A$

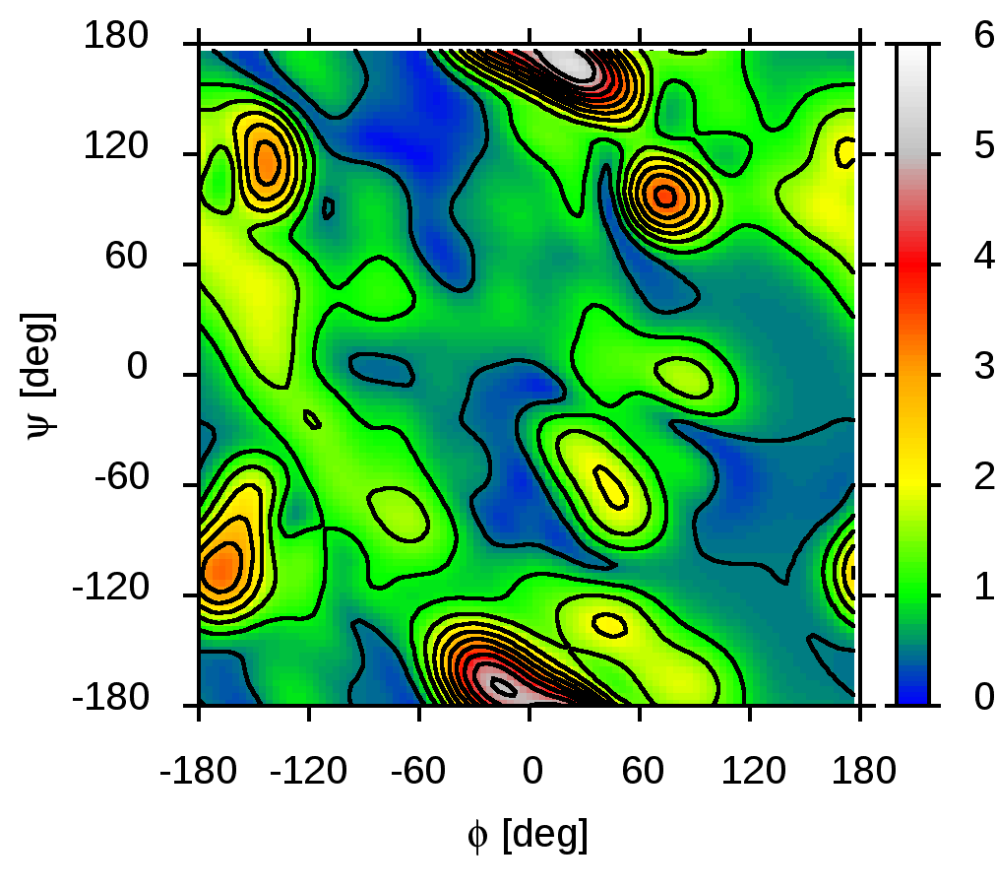


III. Dermatan sulfate (DS)

a. L-IdoA- $\alpha(1 \rightarrow 3)-\beta$-GaINAc

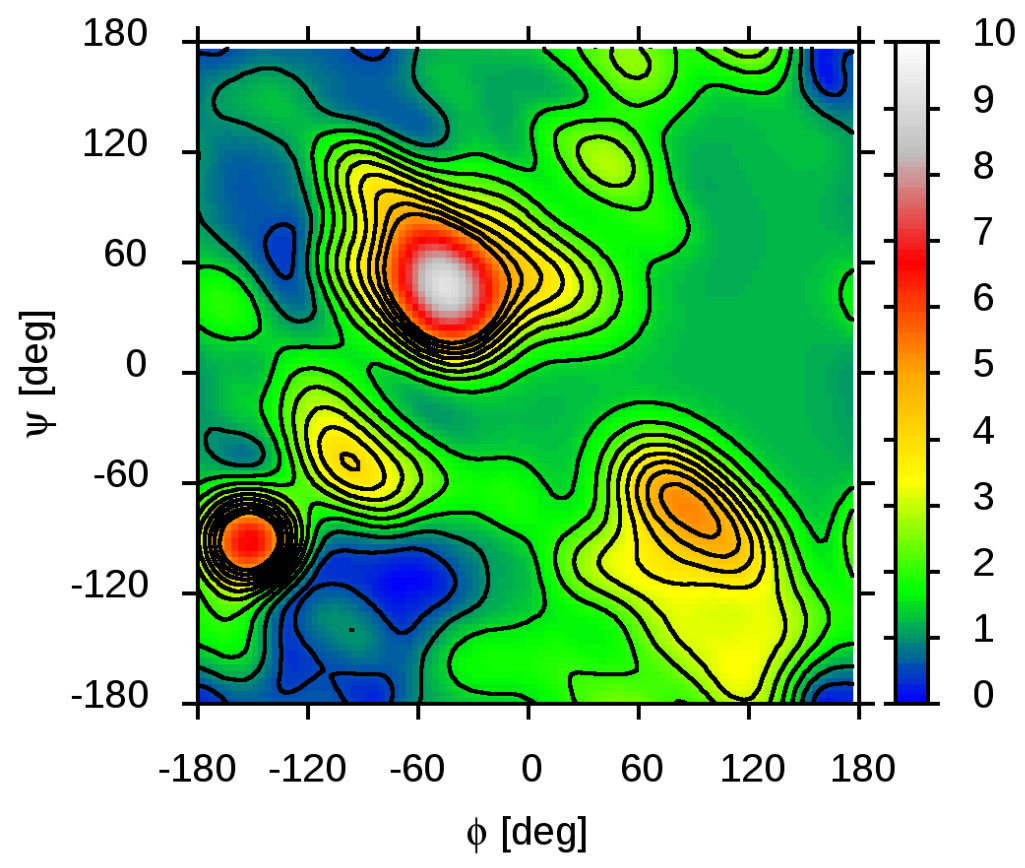

b. GalNAc- $\beta(1 \rightarrow 4)-\alpha-L-I d o A$

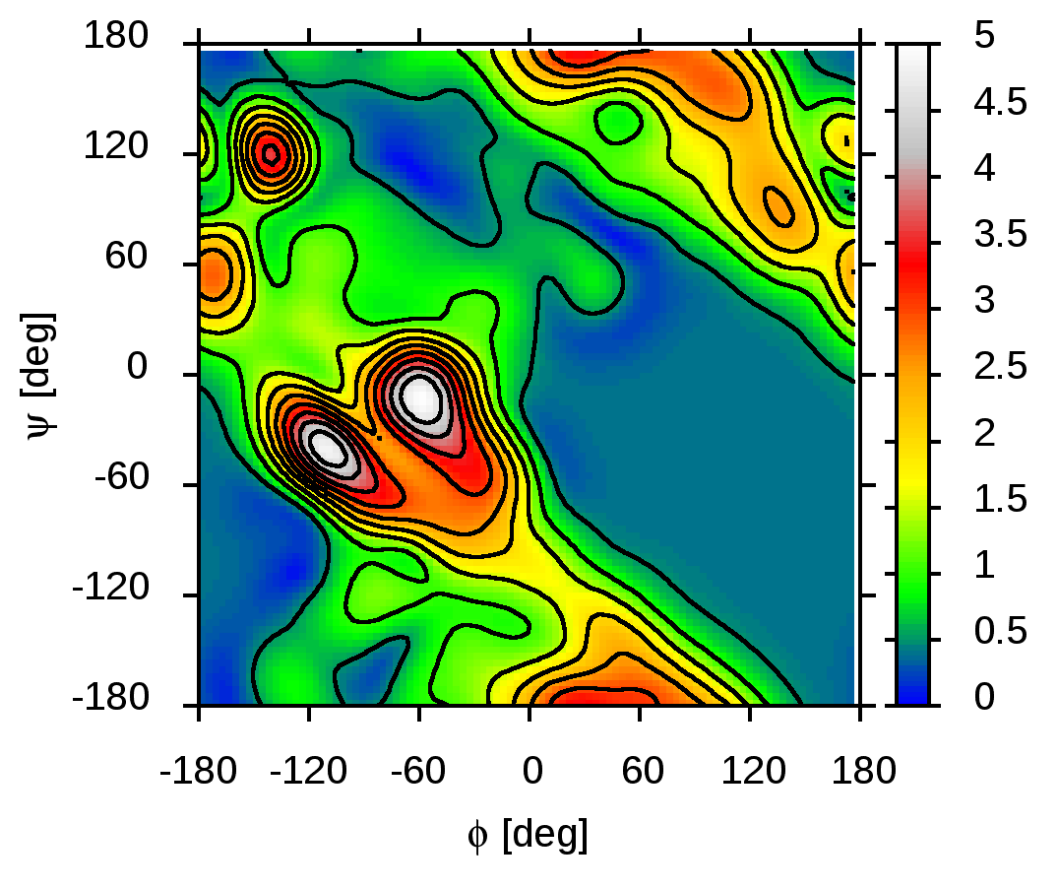


IV. Keratan sulfate (KS)

a. Gal- $\beta(1 \rightarrow 4)-\beta-$ GlcNAc

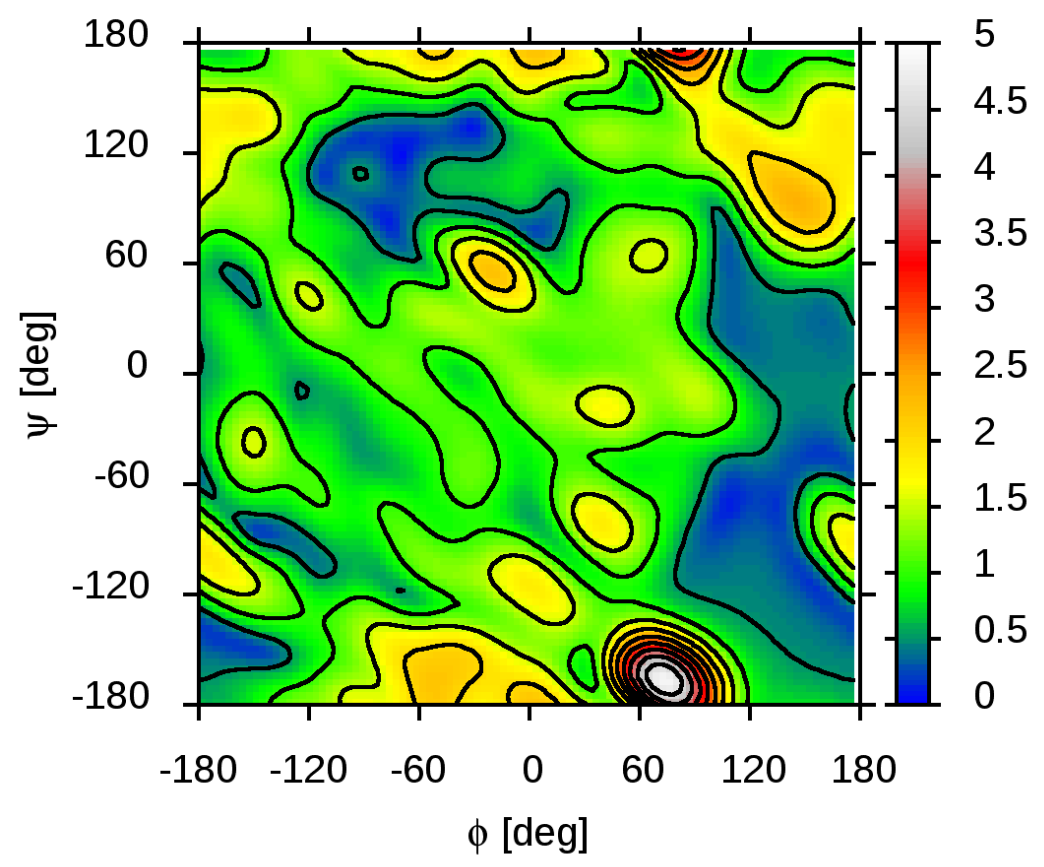

b. GlcNAc- $\beta(1 \rightarrow 3)-\beta-$ Gal

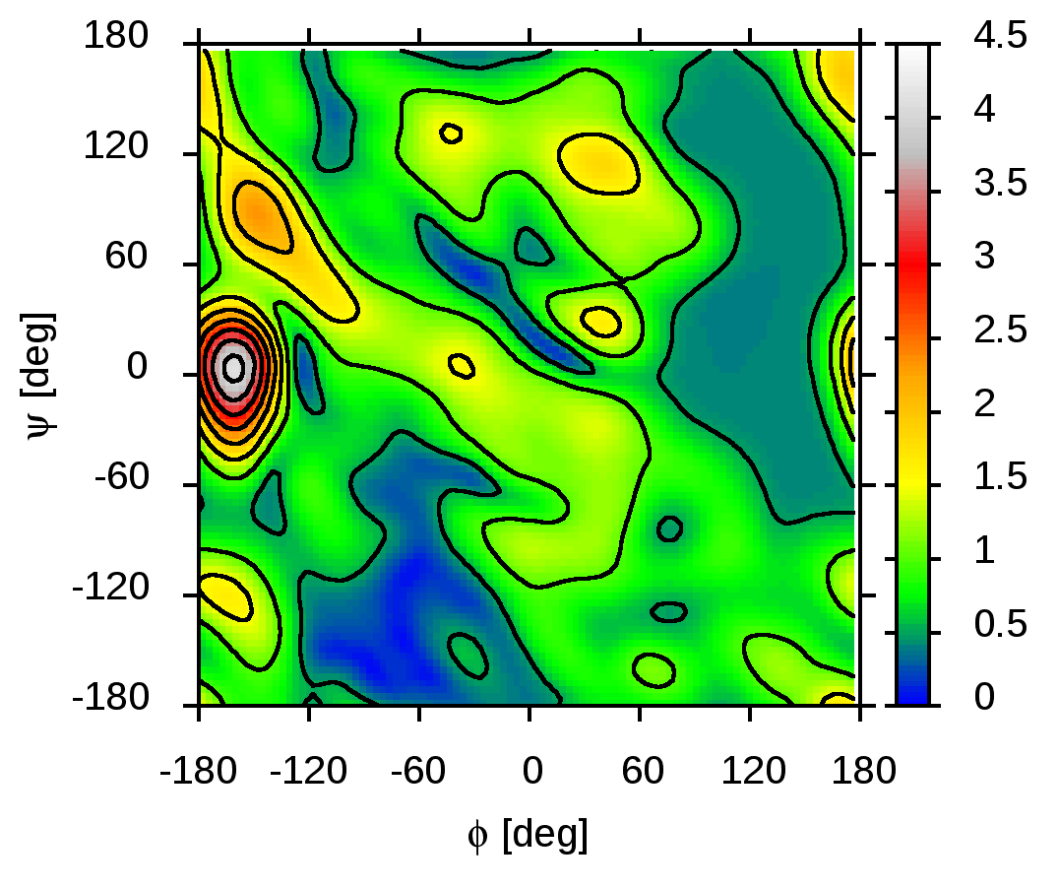


V. Heparin/Heparan sulfate (HP/HS)

a. L-IdoA- $\alpha(1 \rightarrow 4)-\alpha-G \mid c N A c$

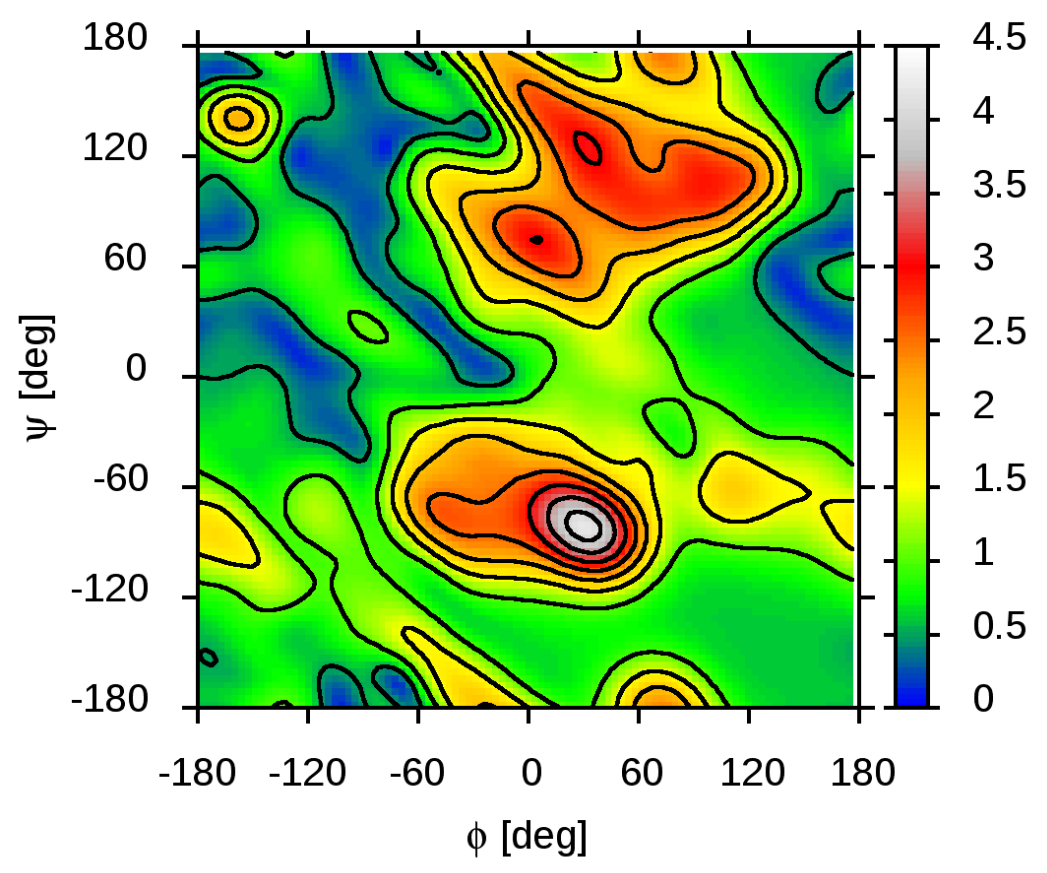

b. GlcNAc- $\alpha(1 \rightarrow 4)-\alpha-L-I d o A$

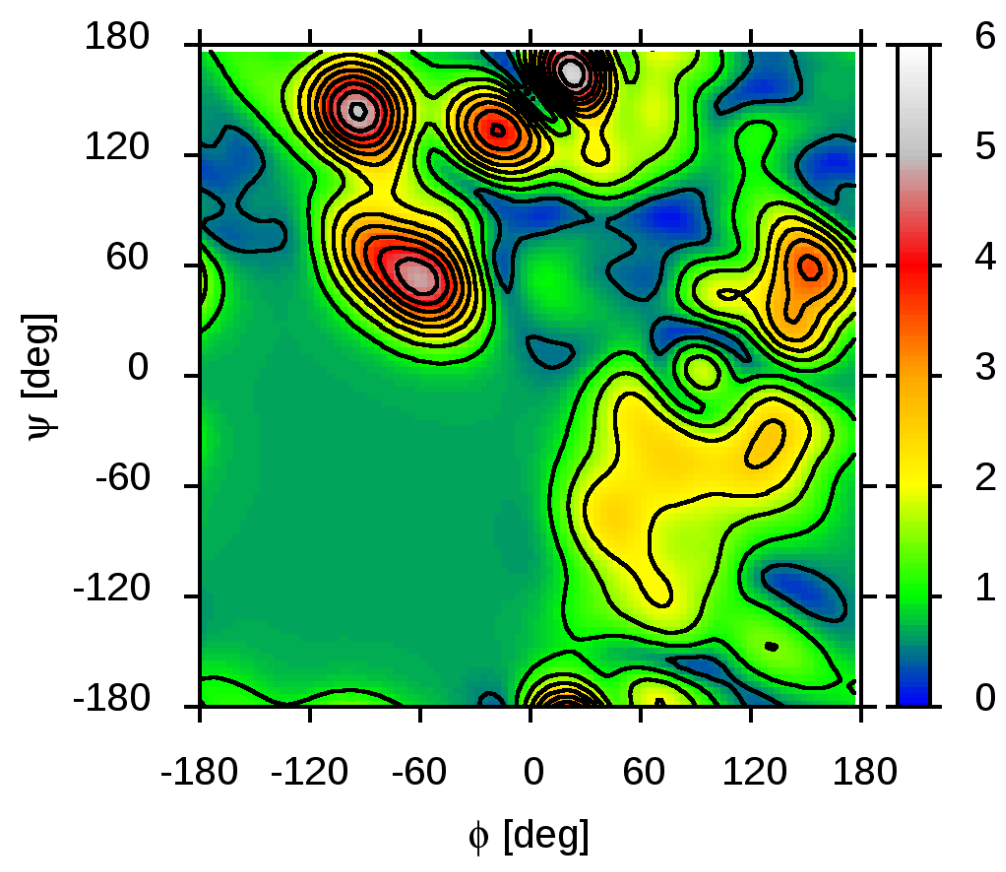


c. $\operatorname{GlcA}-\beta(1 \rightarrow 4)-\alpha-\mathrm{GlcNAc}$

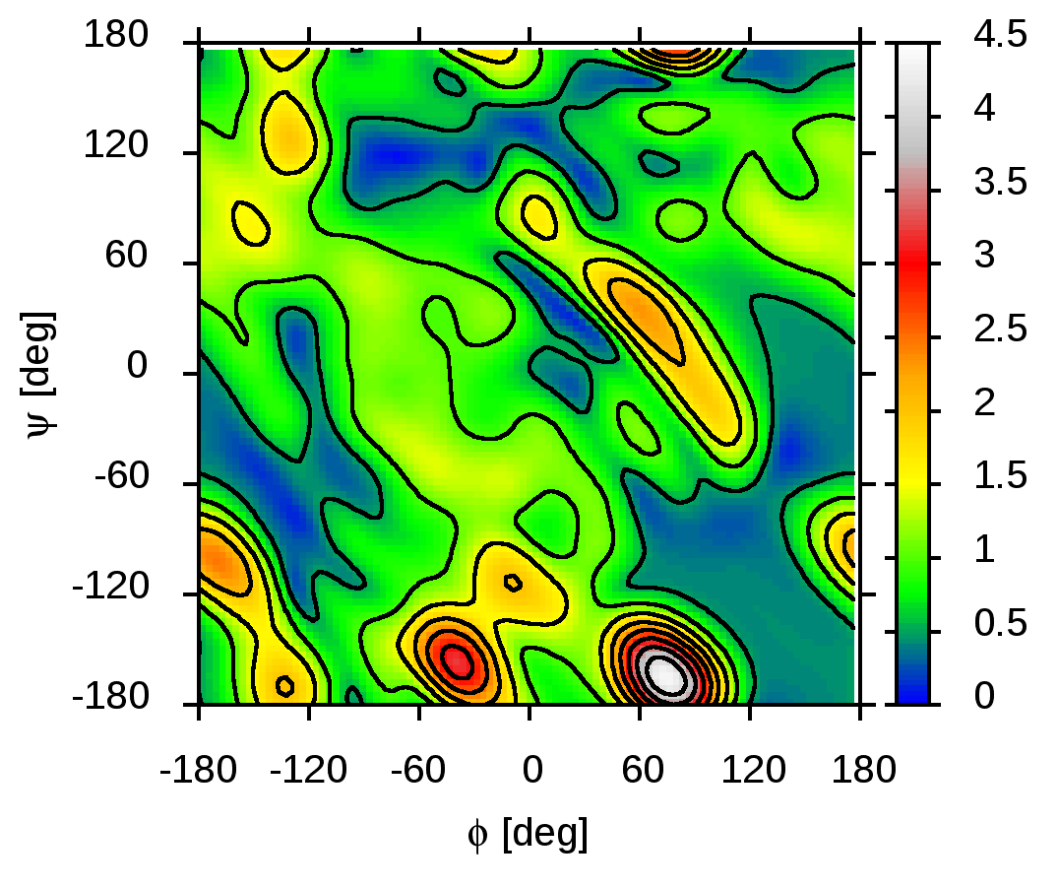

d. GlcNAc- $\alpha(1 \rightarrow 4)-\beta-G l c A$

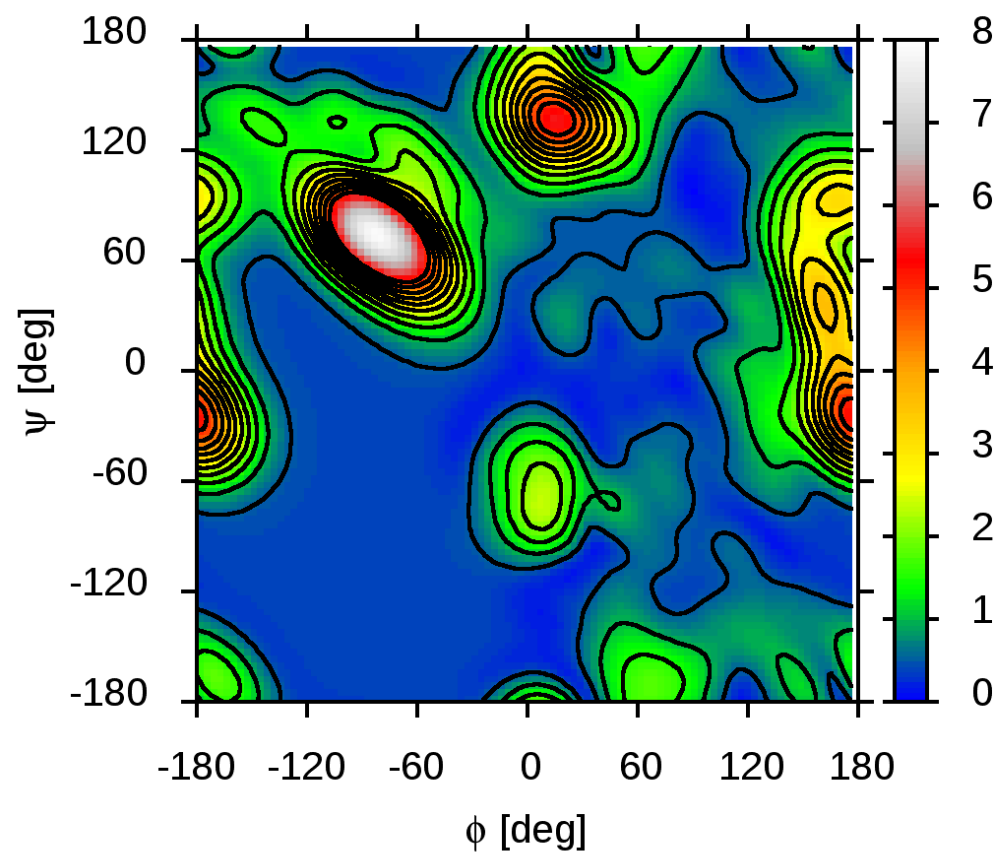


FEMs calculated for selected IdoA-containing compounds with IdoA ring in a non- ${ }^{1} \mathrm{C}_{4}$ conformation (Fig. S4)

L-IdoA- $\alpha(1 \rightarrow 3)-\beta-$ GaINAc:

${ }^{1} \mathrm{C}_{4}:$

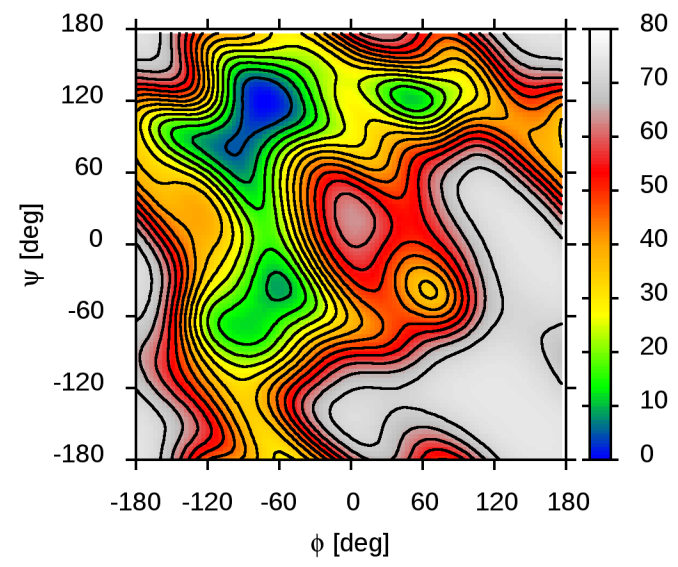

GalNAc- $\beta(1 \rightarrow 4)-\alpha-L-I d o A 2 S:$

${ }^{1} \mathrm{C}_{4}:$

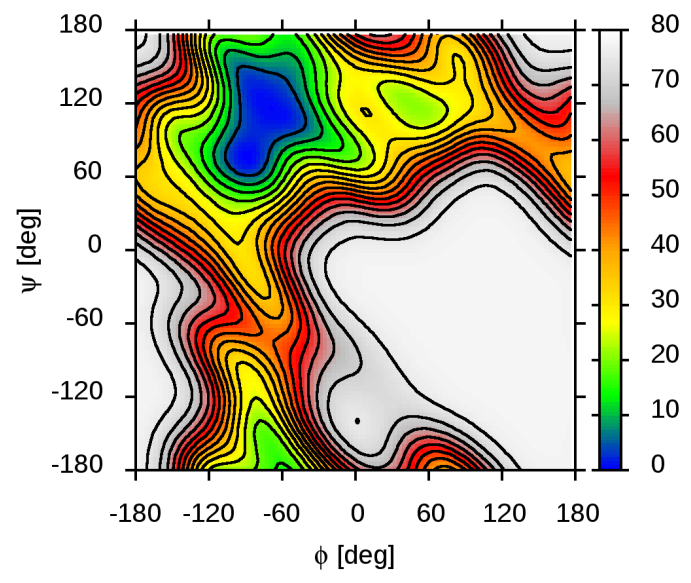

${ }^{4} \mathrm{C}_{1}:$

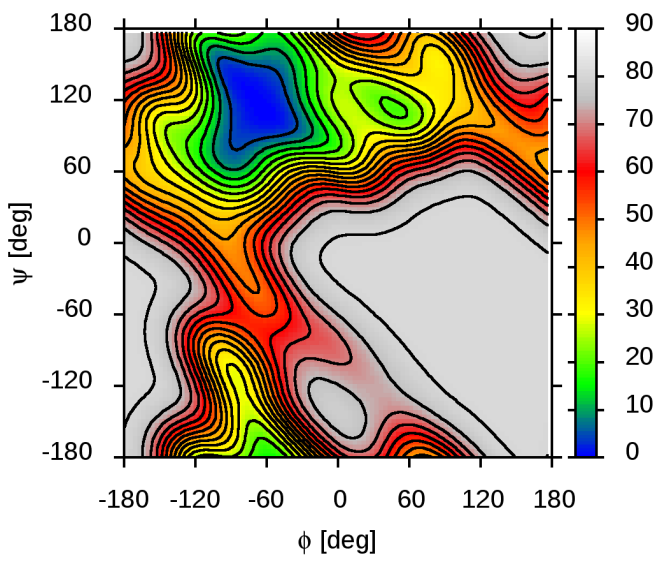

$B / S$ :

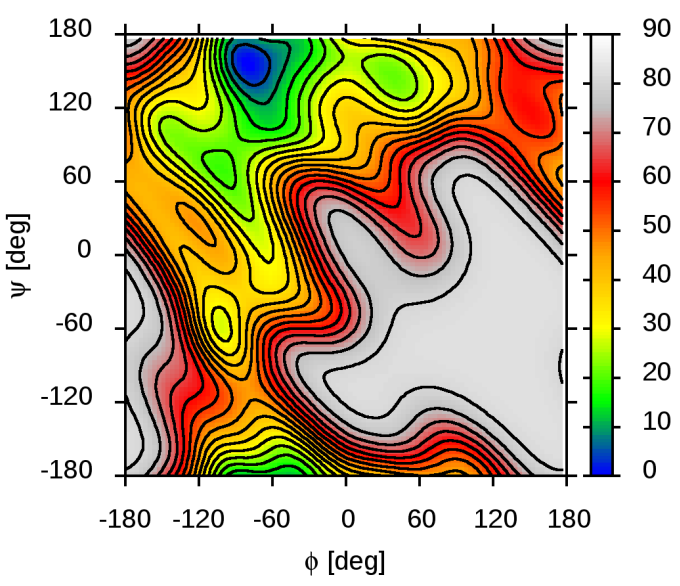


L-IdoA- $\alpha(1 \rightarrow 4)-\alpha-$ GIcNAc:

${ }^{1} \mathrm{C}_{4}:$

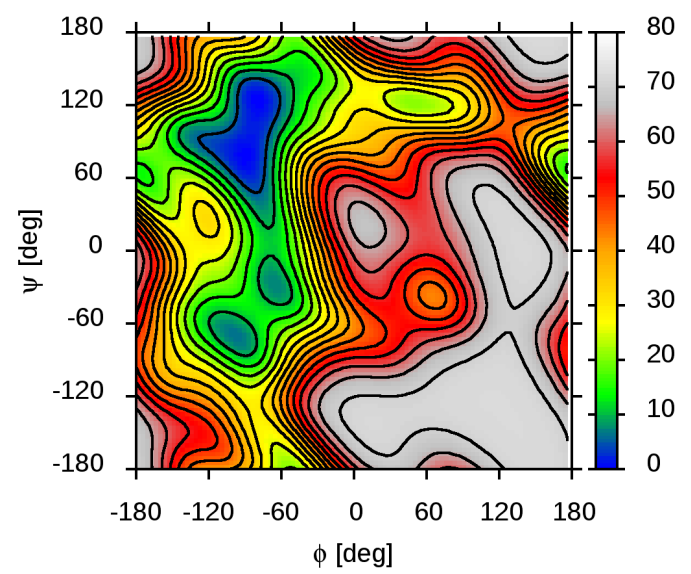

GlcNAc- $\alpha(1 \rightarrow 4)-\alpha-L-I d o A:$

${ }^{1} \mathrm{C}_{4}:$

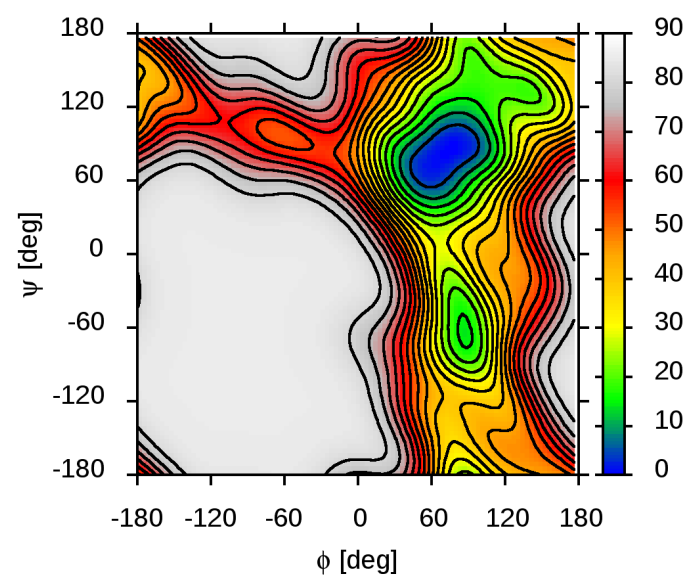

${ }^{4} C_{1}:$

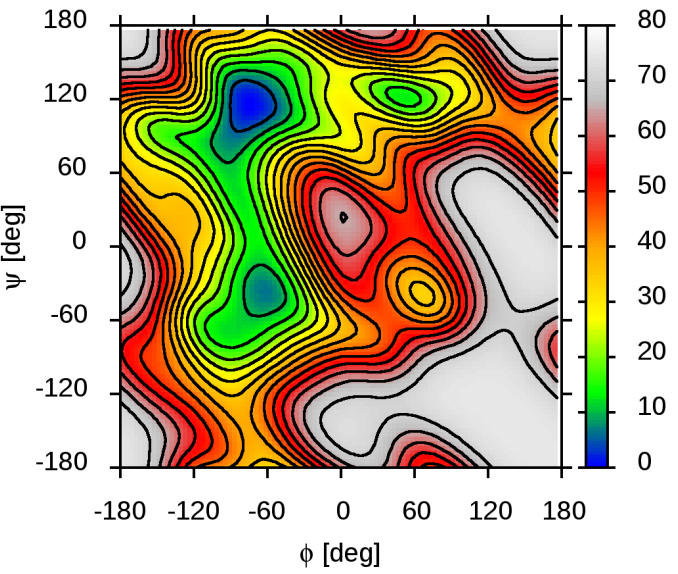

${ }^{4} \mathrm{C}_{1}:$

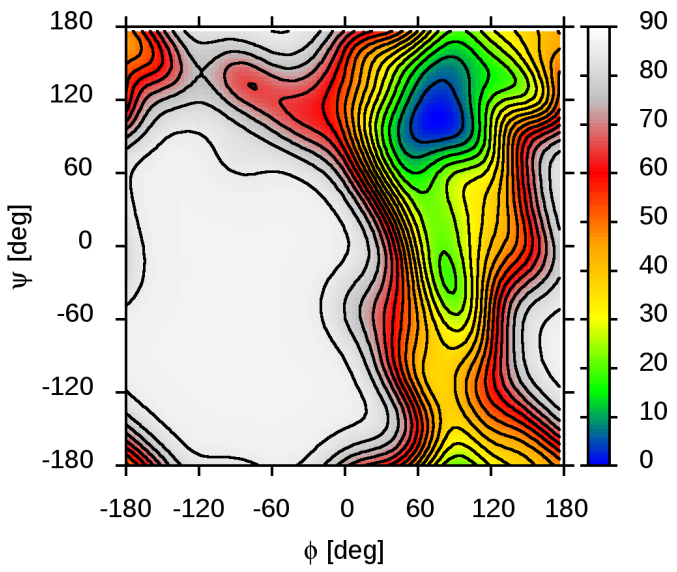




\section{Relation between standard deviations (illustrated in Fig. S3) and energy levels on the corresponding FEMs (Fig. S5)}
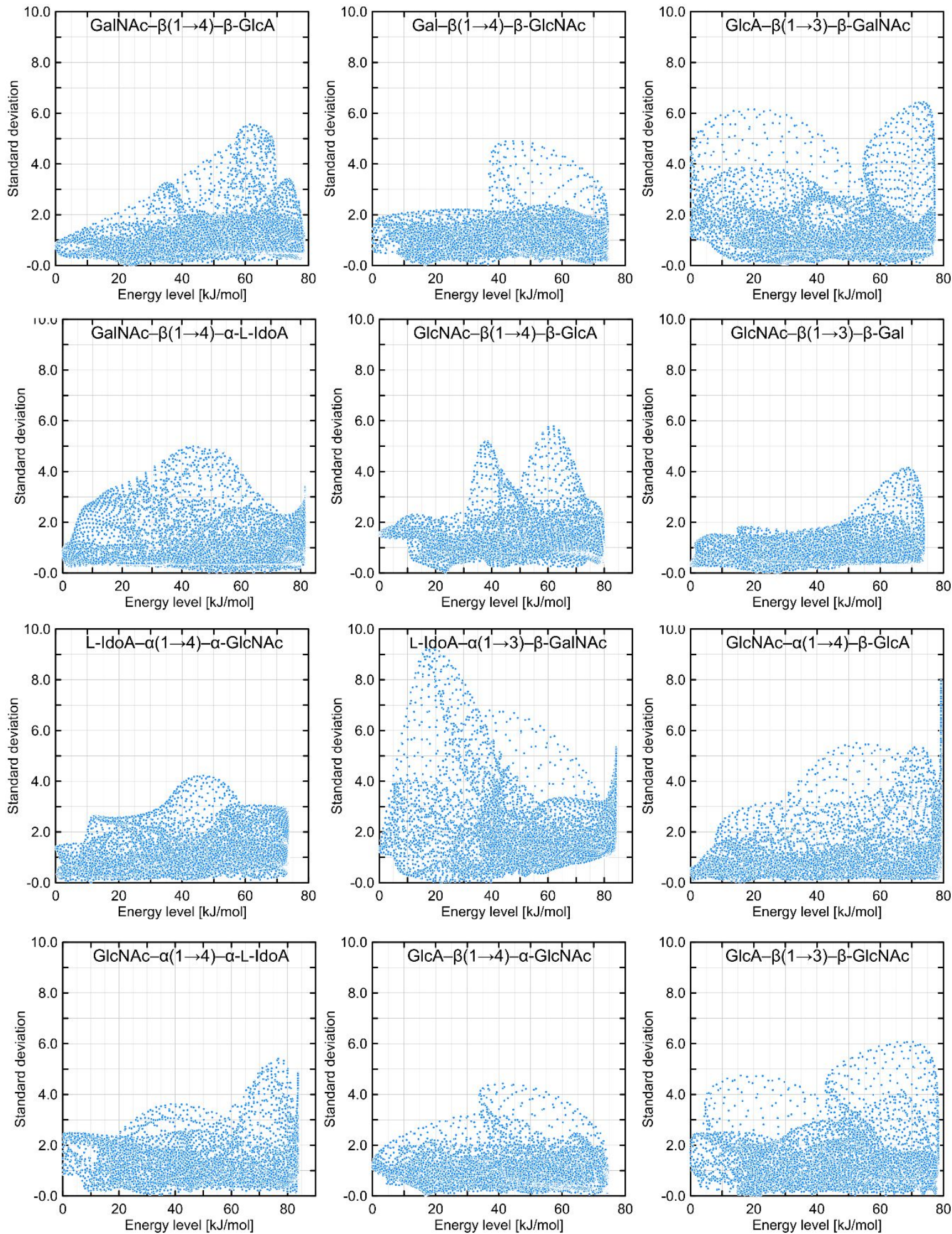